\title{
Methodology for Modeling Building Energy Performance across the Commercial Sector
}

Technical Report NREL/TP-550-41956

March 2008

B. Griffith, N. Long, P. Torcellini, and R. Judkoff National Renewable Energy Laboratory

D. Crawley and J. Ryan

U.S. Department of Energy 


\section{Methodology for Modeling Building Energy Performance across the Commercial Sector}

Technical Report NREL/TP-550-41956

March 2008

B. Griffith, N. Long, P. Torcellini, and R. Judkoff National Renewable Energy Laboratory

D. Crawley and J. Ryan

U.S. Department of Energy

Prepared under Task No. BEC7.1006

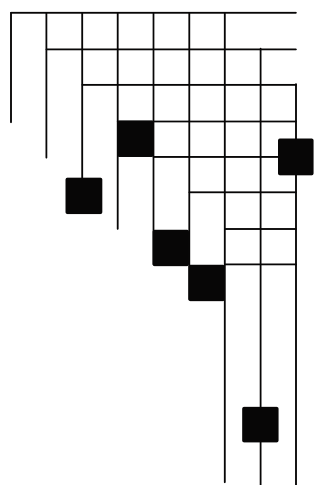

National Renewable Energy Laboratory

1617 Cole Boulevard, Golden, Colorado 80401-3393

303-275-3000 • www.nrel.gov

Operated for the U.S. Department of Energy

Office of Energy Efficiency and Renewable Energy

by Midwest Research Institute • Battelle

Contract No. DE-AC36-99-G010337 


\section{NOTICE}

This report was prepared as an account of work sponsored by an agency of the United States government. Neither the United States government nor any agency thereof, nor any of their employees, makes any warranty, express or implied, or assumes any legal liability or responsibility for the accuracy, completeness, or usefulness of any information, apparatus, product, or process disclosed, or represents that its use would not infringe privately owned rights. Reference herein to any specific commercial product, process, or service by trade name, trademark, manufacturer, or otherwise does not necessarily constitute or imply its endorsement, recommendation, or favoring by the United States government or any agency thereof. The views and opinions of authors expressed herein do not necessarily state or reflect those of the United States government or any agency thereof.

Available electronically at http://www.osti.gov/bridge

Available for a processing fee to U.S. Department of Energy and its contractors, in paper, from:

U.S. Department of Energy

Office of Scientific and Technical Information

P.O. Box 62

Oak Ridge, TN 37831-0062

phone: 865.576 .8401

fax: 865.576 .5728

email: mailto:reports@adonis.osti.gov

Available for sale to the public, in paper, from:

U.S. Department of Commerce

National Technical Information Service

5285 Port Royal Road

Springfield, VA 22161

phone: 800.553.6847

fax: 703.605.6900

email: orders@ntis.fedworld.gov

online ordering: http://www.ntis.gov/ordering.htm 


\section{Contents}

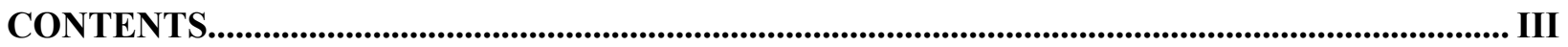

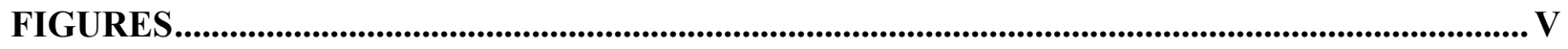

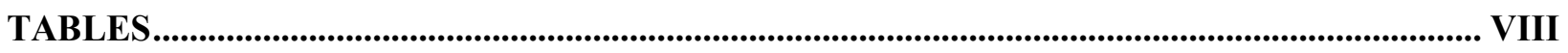

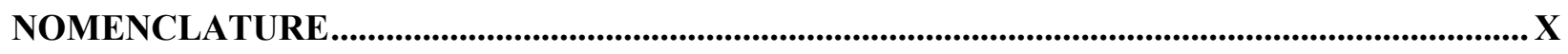

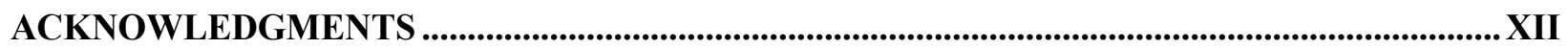

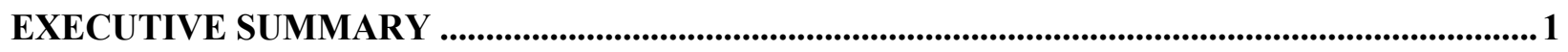

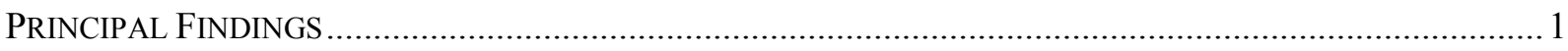

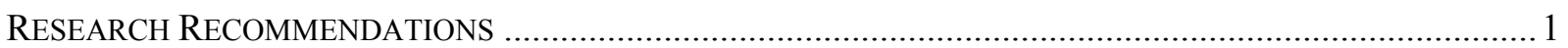

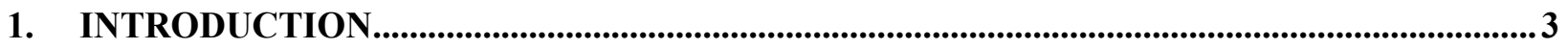

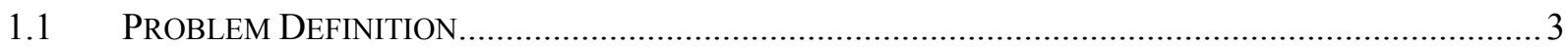

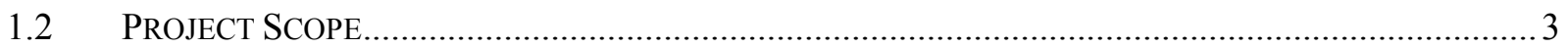

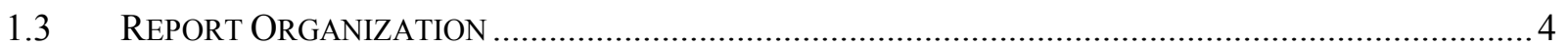

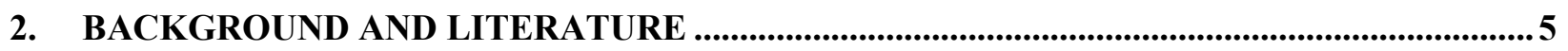

$2.1 \quad 2003$ COMMERCIAL BUILDINGS ENERGY CONSUMPTION SURVEY …........................................... 5

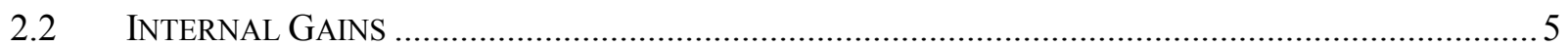

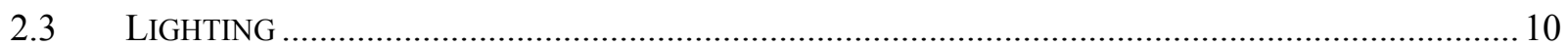

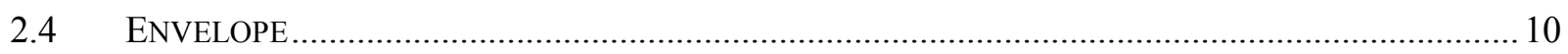

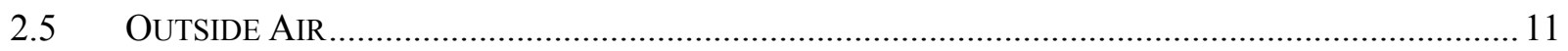

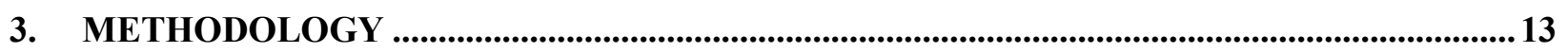

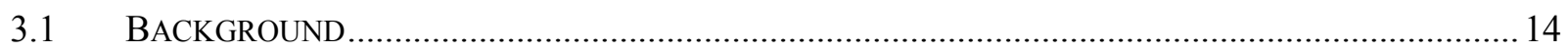

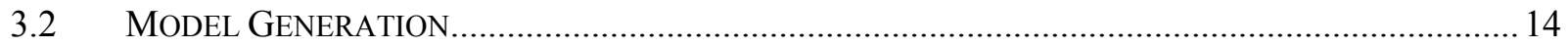

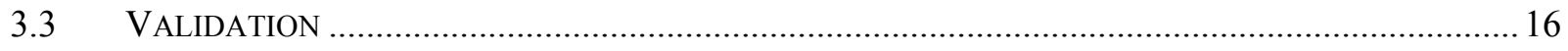

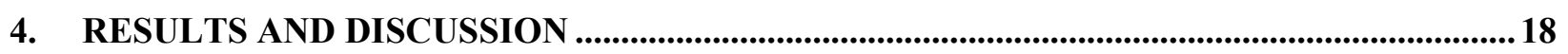

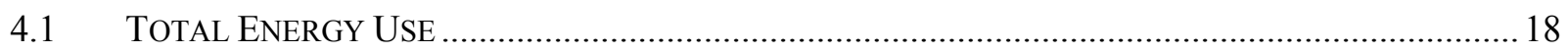

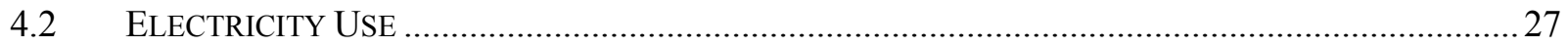

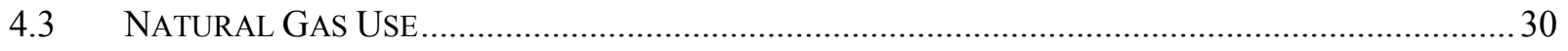

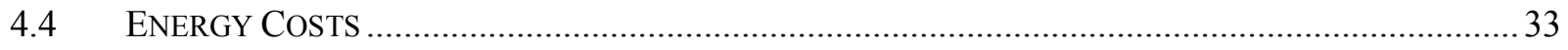

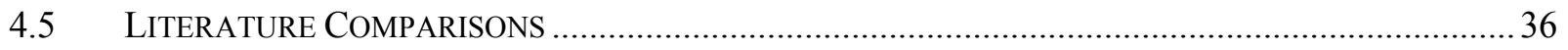

4.6 HISTORICAL WEATHER VERSUS TYPICAL METEOROLOGICAL YEAR .................................... 40

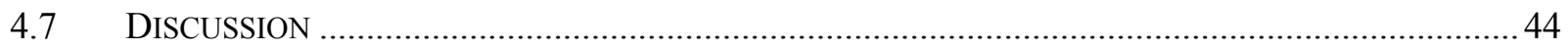

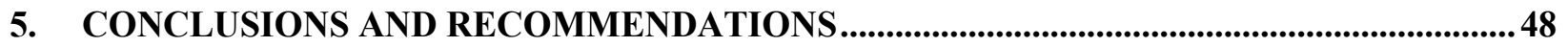

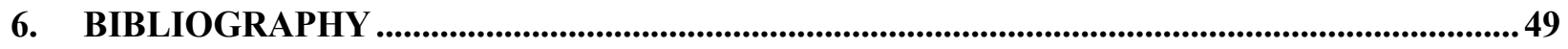

APPENDIX A. EXPANDED RESULTS, TABLES, AND FIGURES ...........................................5 53

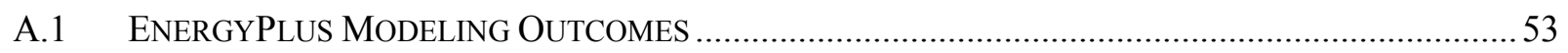

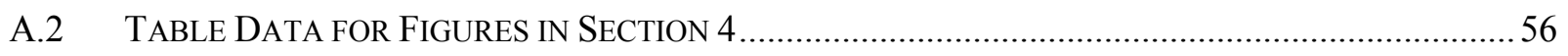




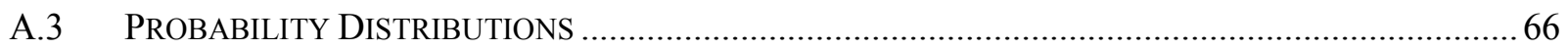

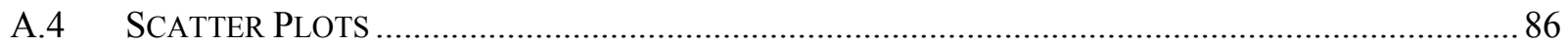

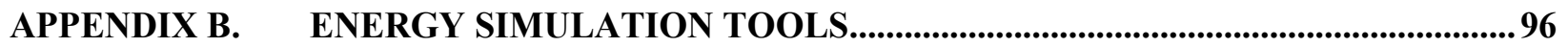

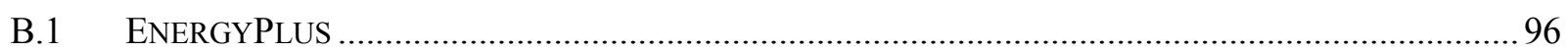

B.2 MODELING FRAMEWORK AND INPUT PARAMETERS …....................................................... 96

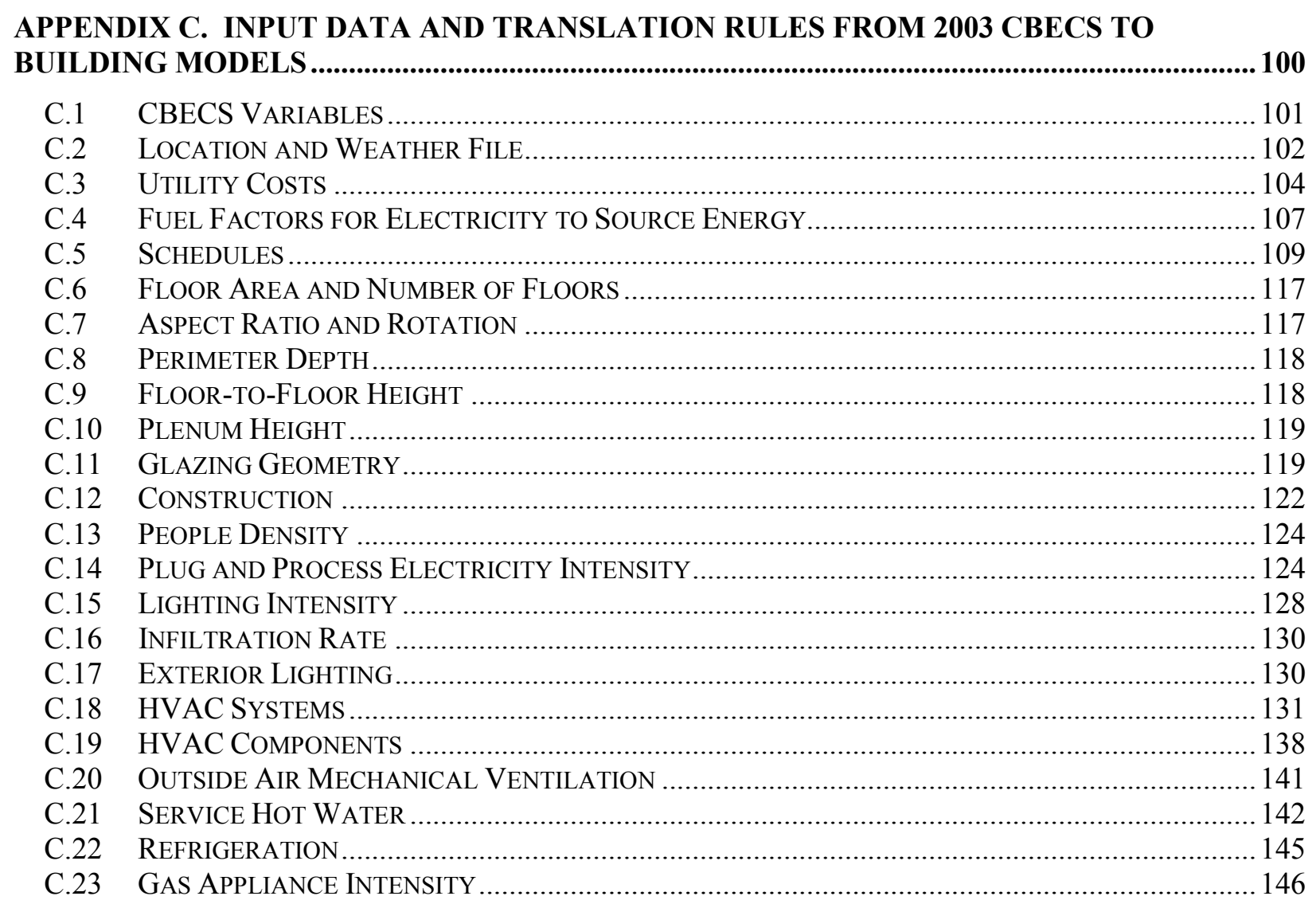




\section{Figures}

Figure 3-1 Overview of Commercial Sector Assessment ................................................................ 13

Figure 4-1 Total EUI: 2003 CBECS Survey and Modeling by Subsector......................................... 18

Figure 4-2 Total EUI: 2003 CBECS Survey and Modeling by Climate Zone................................... 19

Figure 4-3 Map of ASHRAE 169-2006 Climate Zones ............................................................... 19

Figure 4-4 Total EUI: 2003 CBECS Survey and Modeling by Census Division ................................20

Figure 4-5 Electricity Use Intensity: 2003 CBECS Survey and Modeling by Subsector .................... 28

Figure 4-6 Electricity Use Intensity: 2003 CBECS Survey and Modeling by Climate Zone...............29

Figure 4-7 Electricity Use Intensity: 2003 CBECS Survey and Modeling by Census Division .......... 30

Figure 4-8 Natural Gas Use Intensity: 2003 CBECS Survey and Modeling by Subsector ................. 31

Figure 4-9 Natural Gas Use Intensity: 2003 CBECS Survey and Modeling by Climate Zone ............ 32

Figure 4-10 Natural Gas Use Intensity: 2003 CBECS Survey and Modeling by Census Division......... 33

Figure 4-11 Energy Cost Intensity: 2003 CBECS Survey and Modeling by Subsector ......................... 34

Figure 4-12 Energy Cost Intensity: 2003 CBECS Survey and Modeling by Climate Zone................... 35

Figure 4-13 Energy Cost Intensity: 2003 CBECS Survey and Modeling by Census Division .............. 36

Figure 4-14 PDFs of Infiltration Rates from Chan (2006) and Models ................................................ 40

Figure 4-15 Weather Data Scatter Plot: 2003 Historical versus TMY Total EUI ................................ 41

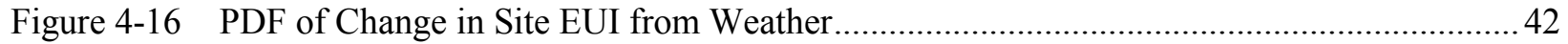

Figure 4-17 Weather Data Scatter Plot: 2003 Historical versus TMY Electricity Use Intensity........... 42

Figure 4-18 Weather Data Scatter Plot: 2003 Historical versus TMY Natural Gas Use Intensity ......... 43

Figure 4-19 Weather Data Scatter Plot: 2003 Historical versus TMY Energy Cost Intensity................ 43

Figure A-1 PDFs for All Buildings: Survey Results in Red, Model Results in Green.......................... 66

Figure A-2 PDFs for All Buildings in Various Climates: Survey Results in Red, Model Results in

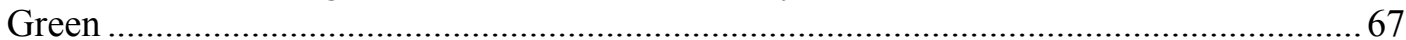

Figure A-3 PDFs for Office Buildings in Various Climates: Survey Results in Red, Model Results

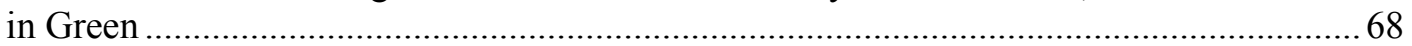

Figure A-4 PDFs for Nonrefrigerated Warehouse Buildings in Various Climates: Survey Results in

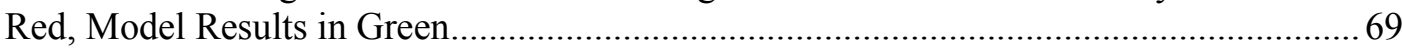

Figure A-5 PDFs for Education Buildings in Various Climates: Survey Results in Red, Model

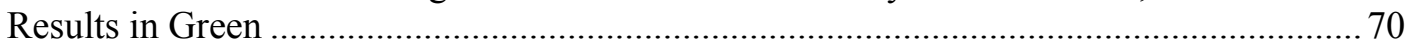

Figure A-6 PDFs for Retail (except Malls) Buildings in Various Climates: Survey Results in Red,

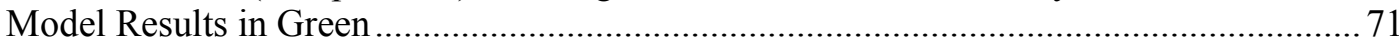

Figure A-7 PDFs for Public Assembly Buildings in Various Climates: Survey results in red, Model

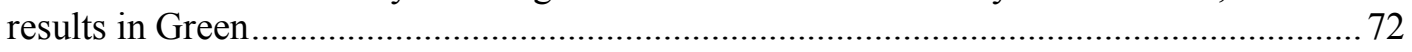

Figure A-8 PDFs for Service Buildings in Various Climates: Survey Results in Red, Model Results

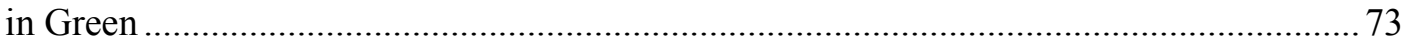

Figure A-9 PDFs for Religious Worship Buildings in Various Climates: Survey Results in Red,

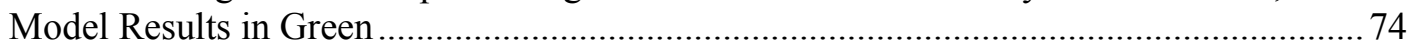

Figure A-10 PDFs for Lodging Buildings in Various Climates: Survey Results in Red, Model

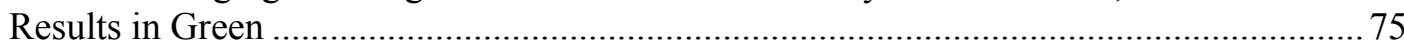

Figure A-11 PDFs for Food Services Buildings in Various Climates: Survey Results in Red, Model

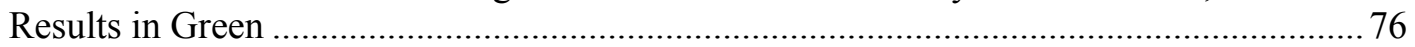

Figure A-12 PDFs for Inpatient Health Care Buildings in Various Climates: Survey Results in Red,

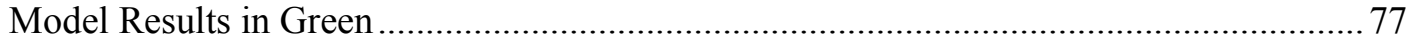

Figure A-13 PDFs for Public Order and Safety Buildings in Various Climates: Survey Results in

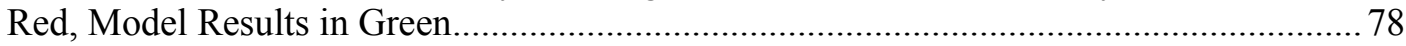

Figure A-14 PDFs for Food Sales Buildings in Various Climates: Survey Results in Red, Model Results in Green 
Figure A-15 PDFs for Outpatient Health Care Buildings in Various Climates: Survey Results in Red, Model Results in Green...

Figure A-16

PDFs for Vacant Buildings in Various Climates: Survey Results in Red, Model Results in Green

Figure A-17 PDFs for Other Buildings in Various Climates: Survey Results in Red, Model Results in Green ...

PDFs for Skilled Nursing Buildings in Various Climates: Survey Results in Red, Model

Results in Green ....

Figure A-19 PDFs for Laboratory Buildings in Various Climates: Survey Results in Red, Model Results in Green .....

Figure A-20

PDFs for Refrigerated Warehouse Buildings in Various Climates: Survey Results in

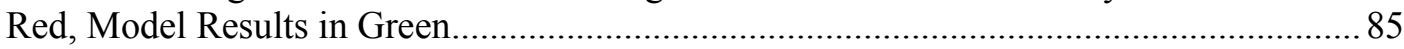

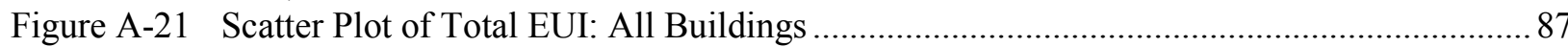

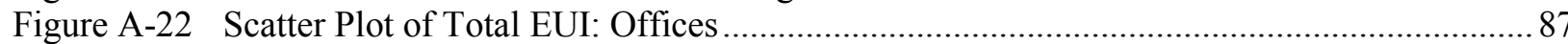

Figure A-23 Scatter Plot of Total EUI: Nonrefrigerated Warehouse ….............................................. 87

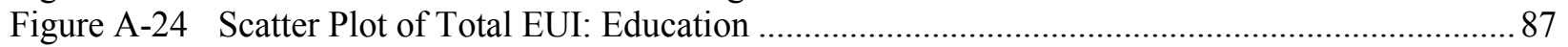

Figure A-25 Scatter Plot of Total EUI: Retail (except Malls) …........................................................ 88

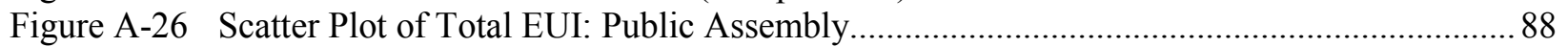

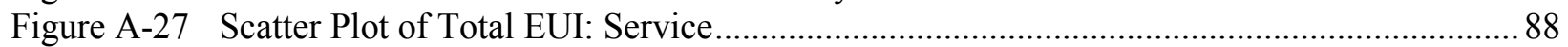

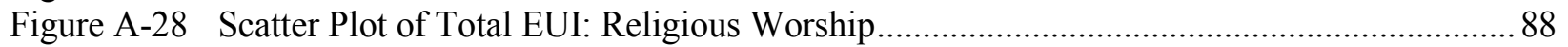

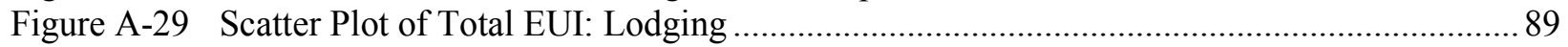

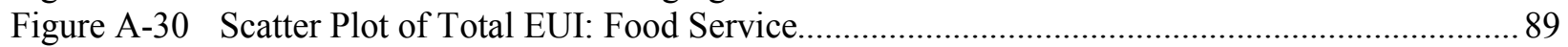

Figure A-31 Scatter Plot of Total EUI: Health Care (Inpatient) .......................................................... 89

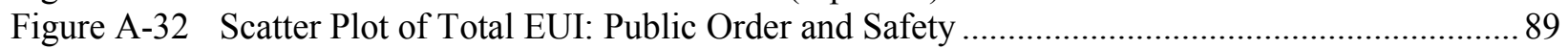

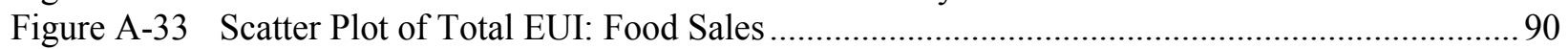

Figure A-34 Scatter Plot of Total EUI: Health Care (Outpatient) .................................................. 90

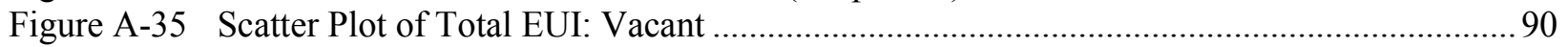

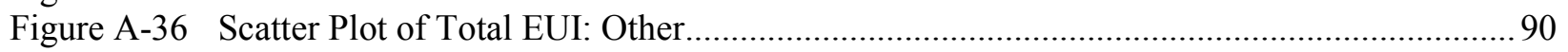

Figure A-37 Scatter Plot of Total EUI: Skilled Nursing ............................................................ 91

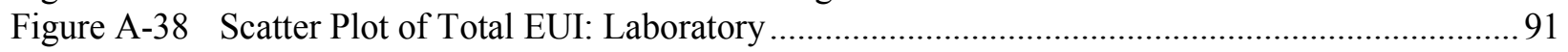

Figure A-39 Scatter Plot of Total EUI: Refrigerated Warehouse ........................................................ 91

Figure A-40 Scatter Plot of Total EUI: Climate Zone 1A ................................................................ 91

Figure A-41 Scatter Plot of Total EUI: Climate Zone 2A …................................................................ 92

Figure A-42 Scatter Plot of Total EUI: Climate Zone 2B ............................................................... 92

Figure A-43 Scatter Plot of Total EUI: Climate Zone 3A ........................................................... 92

Figure A-44 Scatter Plot of Total EUI: Climate Zone 3B .......................................................... 92

Figure A-45 Scatter Plot of Total EUI: Climate Zone 3C ............................................................... 93

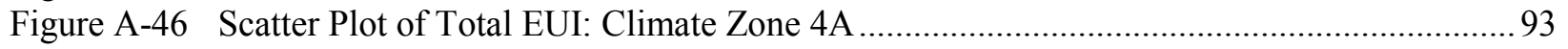

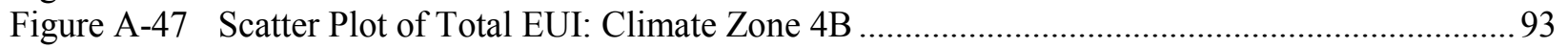

Figure A-48 Scatter Plot of Total EUI: Climate Zone 4C .......................................................... 93

Figure A-49 Scatter Plot of Total EUI: Climate Zone 5A ............................................................. 94

Figure A-50 Scatter Plot of Total EUI: Climate Zone 5B ............................................................. 94

Figure A-51 Scatter Plot of Total EUI: Climate Zone 6A ............................................................. 94

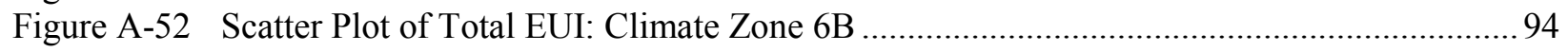

Figure A-53 Scatter Plot of Total EUI: Climate Zone 7 ........................................................... 95

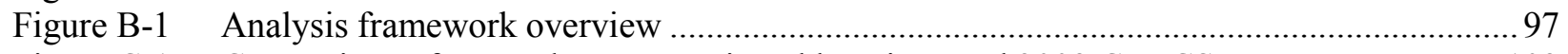

Figure C-1 Comparison of HDDs between assigned locations and 2003 CBECS ............................ 103

Figure C-2 Comparison of CDDs between assigned locations and 2003 CBECS ........................... 103

Figure C-3 Analysis of Percent Cooled and Heated Data (PDF for COOLP8 and HEATP8) ............. 131

Figure C-4 Joint Discrete PDF for MAINCL8 and MAINHT8 (Raw; All Buildings Regardless of

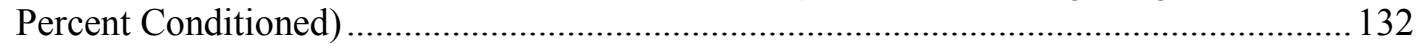


Figure C-5 Discrete PDFs for 2003 CBECS Variables: HVAC Upgraded, Cooling Replaced, Heating Replaced, Maintenance (Raw; All Building Regardless of Percent Conditioned). 


\section{Tables}

Table 2-1 CEUS Results for Plug and Process and Refrigeration Electricity Use Intensities: IP

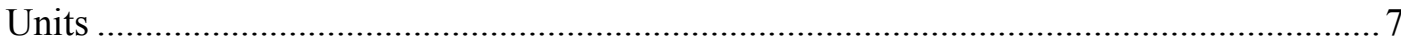

Table 2-2 CEUS Results for Plug and Process and Refrigeration Electricity Use Intensities: SI

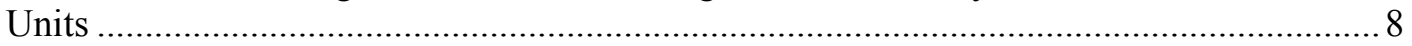

Table 2-3 CEUS Results for Gas End Use Intensities: IP Units.............................................. 9

Table 2-4 CEUS Results for Gas End Use Intensities: SI Units.....................................................9

Table 2-5 U.S. Lighting Market Characterization Results for Lighting Electricity Use..................... 10

Table 2-6 Wall R-Factor Assignments by Building Type, Activity, and Geography (Huang and

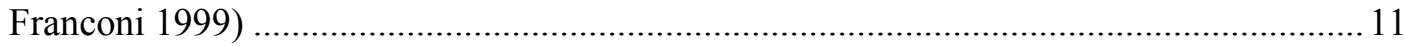

Table 2-7 Roof R-Factor Assignments by Building Type, Activity, and Geography (Huang and

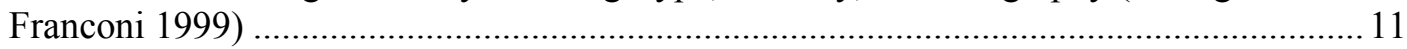

Table 2-8 Measured Outside Air Rates (Turk et al. 1989) .............................................................. 12

Table 3-1 Model Parameter Categories with Sample Parameters ................................................... 14

Table 4-1 2003 CBECS Weighted Mean EUI and Standard Deviation by Subsector and Climate

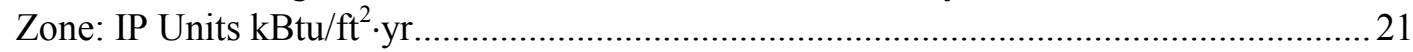

Table 4-2 Existing Stock Models EUI by Subsectors and Climate Zones: IP Units $\left(\mathrm{kBtu} / \mathrm{ft}^{2} \cdot \mathrm{yr}\right)$....... 22

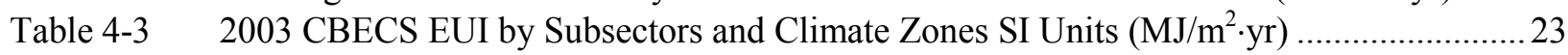

Table 4-4 Existing Stock Models EUI by Subsectors and Climate Zones: SI Units $\left(\mathrm{MJ} / \mathrm{m}^{2} \cdot \mathrm{yr}\right)$......... 24

Table 4-5 Number of Samples and Models by Subsectors and Climate Zones.................................. 25

Table 4-6 Energy Percent Difference between Models and Survey by Subsectors and Climate

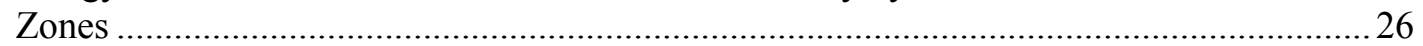

Table 4-7 Plug and Process Electricity Results from CUES Survey and Modeling............................ 37

Table 4-8 Refrigeration Electricity Results from CUES Survey and Modeling.................................. 38

Table 4-9 Interior Lighting Electricity Results from U.S. Lighting Market Characterization and

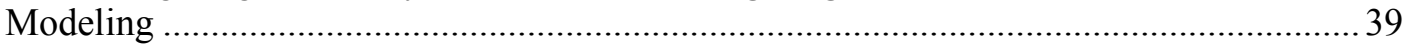

Table 4-10 Site Energy End Use Splits from 2006 Building Energy Data Book and Modeling ............ 40

Table A-1 EnergyPlus Modeling Simulation Run Times by Subsector ............................................. 53

Table A-2 Average Occupied Outside Air Rates in Modeling by Subsector ......................................5

Table A-3 Average Occupied Outside Air Rates in Modeling by Climate Zone: 2003 Historical

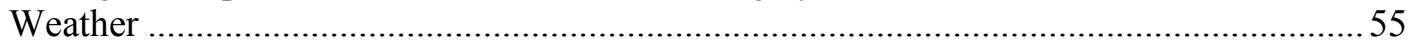

Table A-4 Total EUI Results from 2003 CBECS Survey and Modeling by Subsector ....................... 56

Table A-5 Total EUI Results from CBECS Survey and Modeling by Climate Zone and Weather

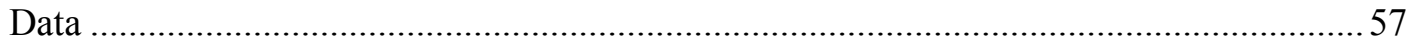

Table A-6 Total EUI Results from CBECS Survey and Modeling by Census Division.......................57

Table A-7 Electricity EUI Results from 2003 CBECS Survey and Modeling by Subsector ................58

Table A-8 Electricity EUI Results from CBECS Survey and Modeling by Climate Zone and

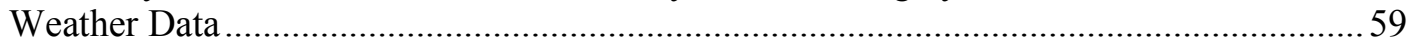

Table A-9 Electricity EUI Results from CBECS Survey and Modeling by Census Division...............60

Table A-10 Natural Gas EUI Results from 2003 CBECS Survey and Modeling by Subsector.............. 61

Table A-11 Natural Gas EUI Results from CBECS Survey and Modeling by Climate Zone and

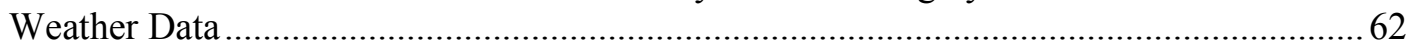

Table A-12 Natural Gas EUI Results from CBECS Survey and Modeling by Census Division ............. 63

Table A-13 Total Energy Cost Intensity Results from 2003 CBECS Survey and Modeling by

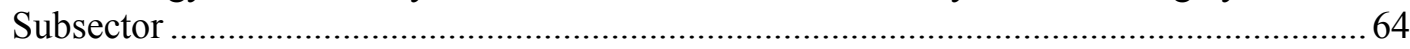

Table A-14 Total Energy Cost Intensity Results from CBECS Survey and Modeling by Climate

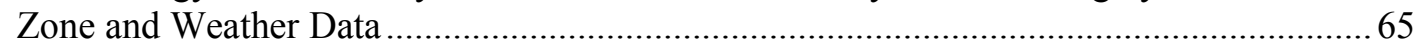


Table A-15 Total Energy Cost Intensity Results from CBECS Survey and Modeling by Census

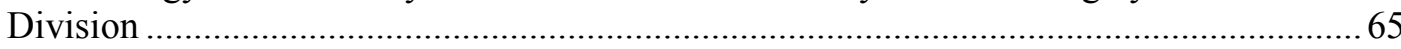

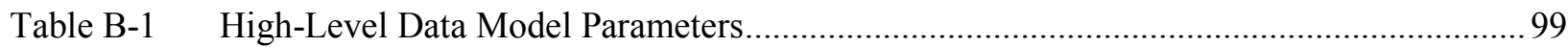

Table C-1 Input Parameter Categories with Sample Parameters ................................................... 100

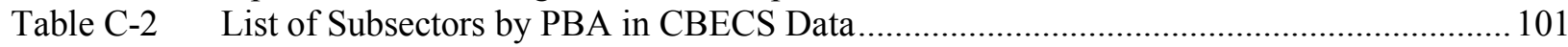

Table C-3 List of Important Variables in CBECS Data.................................................................. 101

Table C-4 Summary of Location Assignments for All Buildings in 2003 CBECS .......................... 102

Table C-6 Utility Rate Assignments for Natural Gas Average Retail Prices for Commercial

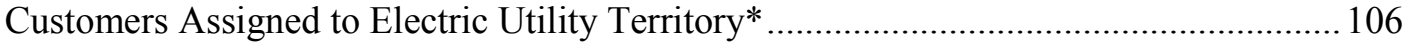

Table C-7 Site-to-Source Conversion Factors for Electricity ...................................................... 108

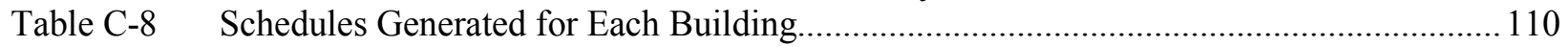

Table C-9 CBECS Variables Used To Determine Hours of Operation ........................................... 111

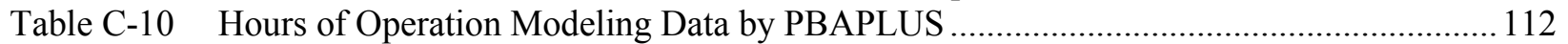

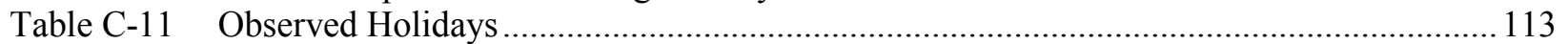

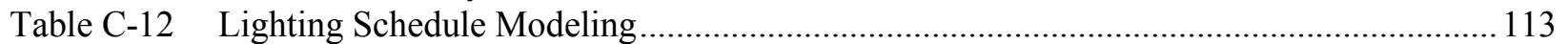

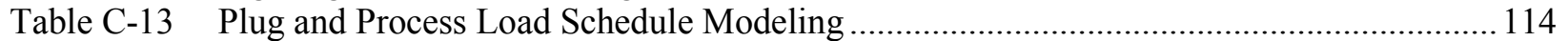

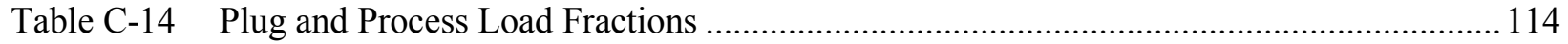

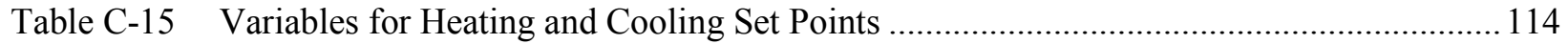

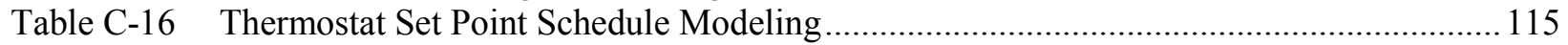

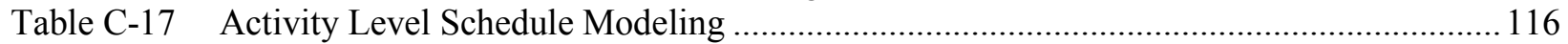

Table C-18 Aspect Ratio Assignments by Building Shape.......................................................... 118

Table C-19 Floor-to-Floor Height Assignments by Subsector .......................................................... 119

Table C-20 Glazing Fraction Assignments ..................................................................................... 120

Table C-21 Glazing Fraction Assignments by PBA (Used When No Data Are Available for Percent

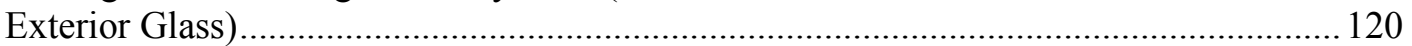

Table C-22 Variables Used To Determine Window Constructions ................................................ 123

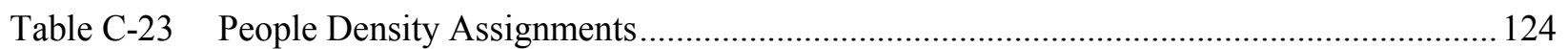

Table C-24 Mean Nominal Peak Power Levels of Surveyed Devices .............................................. 125

Table C-25 Plug and Process Peak Power Levels by PBAPLUS ....................................................... 127

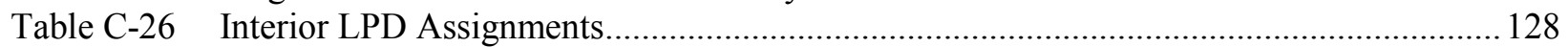

Table C-27 Interior Lighting Schedule Diversity Factors ................................................................... 129

Table C-29 Number of Buildings for Each Combination of MAINCL8 and MAINHT8 (Filtered for

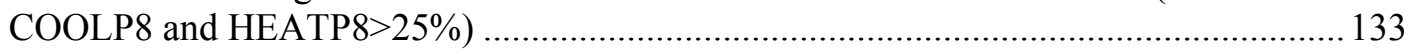

Table C-30 Mappings from MAINCL8 and MAINHT8 to Number of HVAC System as Modeled

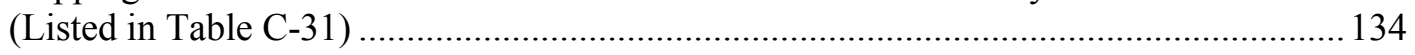

Table C-31 Modeled HVAC System Configurations with Mappings to CBECS Variables................. 134

Table C-32 DX Coil COP Values at Time of Initial Installation for Different Size Ranges ................ 139

Table C-33 Chiller COP Values at Time of Initial Installation for Different Size Ranges ................... 139

Table C-34 Boiler Efficiency Values at Time of Initial Installation ................................................. 140

Table C-35 Heat Pump COP Values at Time of Initial Installation for Different Size Ranges ............ 140

Table C-36 Constant Volume Fan Efficiency Values at Time of Initial Installation............................ 140

Table C-37 Variable-Volume Fan Efficiency Values at Time of Initial Installation ........................... 141

Table C-38 Natural Gas Heating Coil Efficiency Values at Time of Initial Installation ..................... 141

Table C-39 Default Outside Air Mechanical Ventilation Rates by PBA ........................................... 142

Table C-40 SHW Inputs per Person per Day by PBA: SI Units ....................................................... 144

Table C-41 SHW Inputs per Room per Day for Lodging by PBA: SI Units ................................... 145

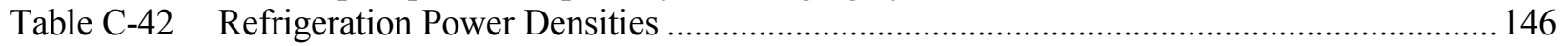

Table C-43 Interior Gas Appliance Intensity Assignments........................................................... 147 


\section{Nomenclature}

AC alternating current

$\mathrm{ACH} \quad$ air changes per hour

AEO Annual Energy Outlook, from EIA

ASHRAE American Society of Heating, Refrigerating and Air-Conditioning Engineers

BT Building Technologies Program within DOE/EERE

CBECS Commercial Buildings Energy Consumption Survey

CDD cooling degree day

CEUS (California) Commercial End-Use Survey

$\mathrm{CH}_{4} \quad$ methane

CHP combined heat and power

$\mathrm{CO}_{2} \quad$ carbon dioxide

$\mathrm{COP} \quad$ coefficient of performance

DC direct current

DOE U.S. Department of Energy

EERE Energy Efficiency and Renewable Energy

EIA Energy Information Administration

EPA U.S. Environmental Protection Agency

EUI energy use intensity

FY Fiscal Year

GDP Gross Domestic Product

HDD heating degree day

HRV heat recovery ventilation (include energy and enthalpy recovery)

HVAC heating, ventilating, and air-conditioning

LBNL Lawrence Berkeley National Laboratory

LPD lighting power density

$\mathrm{NO}_{\mathrm{x}} \quad$ oxides of nitrogen

NREL National Renewable Energy Laboratory

PDF probability density function

PV photovoltaic

R\&D research and development

Sector Energy consumption in the United States is classified by three sectors: transportation, buildings, and industrial. 
SEDS State Energy Consumption, Price, and Expenditure Estimates

Subsector Disaggregation of the sector energy use. As defined by the Commercial Buildings

Energy Consumption Survey (CBECS; EIA 2002), there are 20 subsectors, or principle building activities, in commercial buildings. These include retail, office, and food sales buildings, among others. Complete listings are given throughout this report (see, for example Table 4-1).

TAP Tariff Analysis Project. (See http://tariffs.lbl.gov)

TDD tubular daylighting device

TMY typical meteorological year

TOU time of use

VAV variable air volume

ZEB Zero-energy building (or net-zero energy building). A building with net-zero energy consumption over one year. Assumed to be based on net site energy use. 


\section{Acknowledgments}

This document was prepared under the auspices of the Building Technologies (BT) Program within the Office of Energy Efficiency and Renewable Energy (EERE) at the U.S. Department of Energy (DOE). The authors would like to thank Stefanie Woodward for editing. Special thanks are extended to the following reviewers for their time, attention, and thoughtful contributions to this report: Kurt Roth, Kyle Benne, and Dan Macumber. 


\section{Executive Summary}

This report uses EnergyPlus simulations of each building in the 2003 Commercial Buildings Energy Consumption Survey (CBECS) to document and demonstrate bottom-up methods of modeling the entire U.S. commercial buildings sector (EIA 2006). CBECS survey results provide the most robust set of data available on the energy performance of the national stock of buildings. The ability to use a wholebuilding simulation tool to model the entire sector is of interest because the energy models enable us to answer subsequent "what-if" questions that involve technologies and practices related to energy. This report documents how the whole-building models were generated from the building characteristics in 2003 CBECS and compares the simulation results to the survey data for energy use.

\section{Principal Findings}

- Methods for generating a large population of detailed EnergyPlus models from the data available about building characteristics from 2003 CBECS were developed to implement a mixture of literature data, assumptions, defaults, and probabilistic assignments. Appendix $\mathrm{C}$ of this report documents how data from 2003 CBECS were translated into building descriptions for forward modeling in EnergyPlus.

- Results from the set of 4,820 models are compared to the 2003 survey and overall agreement in site (delivered) energy use intensity is $12 \%$, which is deemed acceptable given the level of scatter in the survey data.

- A by-product of the effort to compare model results to the survey results is the unique analyses of the 2003 CBECS public use data. For example, the modeling effort produced climate zone estimates for the sample buildings so survey results can be organized by climate zone. Appendix A contains plots of survey data in the form of probability density functions and scatter plots, which show that energy use intensity is broadly distributed. The masking of data and shifting of floor area in the samples may have introduced additional errors in area-normalized use intensities that were calculated from public use data.

- The sample size of 4,820 is adequate for characterizing some, but not all, subsets of the commercial sector that are formed by principal building activity and climate.

\section{Research Recommendations}

The National Renewable Energy Laboratory's efforts to model the commercial building stock resulted in a number of research recommendations:

- The set of models can serve as the starting point for simulation-based studies of the commercial sector. Such studies require a set of building types and locations. The set developed here has a strong statistical basis because it reuses the sample designed for CBECS. Examples of studies that could be conducted include analysis of retrofit options on existing stock, energy impacts of outside air, and analysis of savings potential in new construction, if one assumes that the distribution of new construction mirrors that of the existing stock.

- The existing stock models could be further refined for future analysis. The current models were based on the building characteristics from 2003 CBECS; the survey results for energy consumption were reserved for validation. Future work could improve the models by forcing the modeled energy consumption to agree with the survey results for each sample. The models would need to be based on the actual sample floor areas rather than on masked data.

- The methodology documented here could be extended to augment survey data in future CBECS. By incorporating EnergyPlus modeling into the analysis and postprocessing of CBECS data, models could facilitate predicting energy end-use distributions, normalizing energy use with 
respect to weather, and synthesizing time series data such as peak power demands and hourly load profiles.

- In addition to typical meteorological year weather data, the National Solar Radiation Database historical weather data are practical for modeling the behavior of commercial buildings with respect to periods of real weather such as for 2003. Being able to switch weather data suggests that simulations could be used to normalize survey results with respect to weather.

- The methodology documented here is computationally intensive and appropriate for large research projects only. For routine or widespread use, we recommend developing smaller sets of benchmark models to represent the commercial sector.

- Results from the modeling for energy consumption estimates by end use differ from similar data in the Building Energy Data Book (DOE 2006). The current study has significantly lower end uses for service hot water and higher end uses for cooling and fans. More research is needed to resolve these discrepancies. The modeling of service water heating would need to be improved before the method could be applied to evaluate water heating technologies. 


\section{Introduction}

\subsection{Problem Definition}

The commercial building sector is responsible for $18 \%$ of U.S. energy use and is the fastest growing demand sector. The Energy Information Agency's (EIA) Annual Energy Outlook 2007 (AEO; EIA 2007) projects growth at $1.5 \%$ per year. This growth will place an additional burden on the nation's energy system and is driven by economic expansion, which leads to increased commercial floor area. Research and policy efforts aimed at improving the energy performance of the commercial sector could benefit from a better understanding of the myriad details of why, how, when, and where energy is used - and how it can be saved.

The survey conducted by EIA in four-year cycles, called the Commercial Building Energy Consumption Survey (CBECS), is used for official government statistics, and is thus an excellent source of real-world data about the energy performance of the commercial sector. The latest survey was conducted for 2003 (2003 CBECS; EIA 2006). CBECS is an important basis for what researchers and analysts know about the commercial sector. However, the types of data collected are fairly limited and do not include temporal details such as peak electricity demand, end uses, or hourly distributions. CBECS helps us to understand the existing stock, but it is less useful for new construction. As a survey, it does not provide predictive capabilities that could answer "what if" questions.

Detailed simulations of the annual energy performance of the buildings can be conducted with computer modeling tools such as EnergyPlus (www.eere.energy.gov/buildings/energyplus/). These models are forward in the sense that they take a description of the building and its operation and predict energy performance over a specified time period such as annually. Such tools model system interactions in detail and enable accurate evaluation of alternative technologies and practices. However, such models are inherently resolved at the level of a single building, which creates challenges in using them for large-scale studies that address the entire United States. To use EnergyPlus for a sector-wide study requires developing a sector model that is composed of a number of individual building models that seek to represent the all types of buildings and locations. Once developed, such sets of EnergyPlus models for the whole sector can answer "what if" questions such as, What is the feasibility of net zero-energy buildings? and What is the value of individual energy savings technologies and practices? However, the sector model needs to be validated so that such methods can be used with greater confidence in subsequent studies.

\subsection{Project Scope}

The principal question being addressed in this report is: "How well do results from a set of EnergyPlus models for the whole sector agree with 2003 CBECS?"

The U.S. Department of Energy Office of Energy Efficiency and Renewable Energy Building Technologies Program (DOE/EERE/BT) conducts research in the area of commercial building integration. This study was conducted to further develop and validate a whole-sector analysis methodology based on large numbers of EnergyPlus simulations. Since the survey results from CBECS are the best sources of real-world data about the commercial sector, these data should be used as a check. Although the National Renewable Energy Laboratory's (NREL) overall research efforts currently focus on new construction, this part of the study focuses on existing commercial stock and allows us to compare model results to the 2003 CBECS survey results in an effort to validate the methodology.

NREL used EnergyPlus to produce a data set of energy performance metrics for commercial buildings in various subsectors, climates, and census divisions. We used the information gathered from these data sets to:

- Determine whether EnergyPlus modeling results are reasonably valid.

- Determine whether the whole-sector modeling methodology represents the commercial sector. 


\subsection{Report Organization}

This report is organized into three tiers that contain increasingly technical content:

- The first tier is the Executive Summary.

- The second tier forms the body of the report, and is presented in five sections.

- Section 1 introduces the problem and methods NREL used in this research.

- Section 2 summarizes the literature we used to help characterize the commercial sector.

- Section 3 summarizes the methods we used in this study to model the existing stock.

- Section 4 presents and discusses selected results from the study.

- Section 5 is a bibliography.

- The third tier presents significantly more technical detail in three appendices.

- Appendix A contains expanded results.

- Appendix B discusses the analysis tools NREL used to perform the study.

- Appendix $\mathrm{C}$ documents translation rules used to develop detailed models from survey data. 


\section{Background and Literature}

This section summarizes the literature and data sources used in the methodology. The primary source of data is EIA's 2003 CBECS. So-called "third party" literature refers to data sources that are external to either EIA's or the authors' current efforts.

\subsection{Commercial Buildings Energy Consumption Survey}

The CBECS is a survey of U.S. buildings that EIA conducts every four years. The latest is the 2003 CBECS. EIA releases a fine-grained data set about the sample buildings for public use. The 2003 CBECS Public Use Microdata set includes data for 4,820 non-mall commercial buildings. A second data set is available that includes malls, but it lacks data on the building characteristics for malls to be included in this study. For each sample, CBECS provides data about building characteristics, including floor area, number of floors, census division, basic climatic design criteria, principal building activity (PBA), number of employees, type of heating and cooling equipment, and weighting factors. The CBECS building characteristics data are the core source used in this study to develop methods for modeling commercial buildings. The CBECS data about energy consumption are compared to model results in an effort to validate the whole-sector modeling approach.

The 2003 CBECS data survey provides the best statistical characterization of the commercial sector available for the United States, but it is not designed to provide the details needed for whole-building energy performance modeling. A methodology was developed to bridge the actual data and the data required for EnergyPlus simulations. Data obtained from separate literature sources, which are the subject of this section, are important to this methodology.

\subsection{Internal Gains}

The California Energy Commission (CEC) commissioned Itron, Inc., to conduct a large study called the California Commercial End-Use Survey, or CEUS, of the commercial sector in California (CEC 2006). The CEUS survey is analogous to EIA's CBECS survey, but focuses on California, specifically the service areas of four major utility companies. CEUS differs from CBECS in several important ways:

- CEUS was not designed with the anonymity that would allow a publicly available, fine-grained data set of the characteristics of individual buildings in the survey to be produced. No data set from CEUS is comparable to the Public Use Microdata from CBECS; there are only aggregated results.

- Building energy performance modeling was closely integrated with the methodology used to conduct the survey. The computer modeling done for CEUS is collected in an analysis framework called "DrCEUS" that is based on the simulation engine DOE 2.2/EQUEST and other proprietary software for non-heating, ventilation, and air-conditioning (HVAC) end uses. Energy model development appears to have had close access to many more details of the individual survey buildings than what are available from 2003 CBECS. Details include monthly billing data (which can be used to calibrate models), event loggers in some buildings to determine occupancy schedules, and more detail about the inventory of the types of non-HVAC devices in the buildings.

- The aggregated results have been transformed to correspond to typical meteorological year (TMY) weather rather than the historical weather from the survey period.

Although the lack of publicly available data limits the usefulness of the study (for those outside of CEC and Itron), there appear to be important lessons (and precedents) for using energy performance simulation as a core part of a methodology for conducting such surveys. The aggregated data from CEUS appear to be the best available for characterizing occupant-driven energy end uses in the commercial sector. To apply the CEUS data, we reason that occupant-driven activities and intensity of use in California commercial buildings are similar to the rest of the U.S. commercial sector and assume that the study will 
benefit by extrapolating the high-quality data for California to the rest of the United States. Table 2-1 and Table 2-2 summarize CEUS's results (from Table 8-3 in CEC 2006) used in this study to develop input data related to plug and process electricity use as well as commercial refrigeration. The CEUS data listed in Table 2-1 and Table 2-2 are used in the models. Plug and process electricity loads are assumed to be the subtotal of office equipment, miscellaneous, process, cooking, air compressors, and motors.

Similarly, Table 2-3 and Table 2-4 summarize CEUS's results (from Table 8-5 in CEC 2006) used here to develop input data related to gas appliance use. 
Table 2-1 CEUS Results for Plug and Process and Refrigeration Electricity Use Intensities: IP Units

\begin{tabular}{|c|c|c|c|c|c|c|c|c|}
\hline $\begin{array}{c}\text { CEUS Building } \\
\text { Type }\end{array}$ & $\begin{array}{c}\text { Office } \\
\text { Equipment } \\
\left(\mathrm{kBtu} / \mathrm{ft}^{2} \cdot \mathrm{yr}\right)\end{array}$ & $\begin{array}{l}\text { Miscellaneous } \\
\left(\mathrm{kBtu} / \mathrm{ft}^{2} \cdot \mathrm{yr}\right)\end{array}$ & $\begin{array}{c}\text { Process } \\
\left(\text { kBtu/ft }^{2} \cdot \mathrm{yr}\right)\end{array}$ & $\begin{array}{l}\text { Cooking } \\
\left(\mathrm{kBtu} / \mathrm{ft}^{2} \cdot \mathrm{yr}\right)\end{array}$ & $\begin{array}{c}\text { Air } \\
\text { Compressor } \\
\left.\text { (kBtu/ft }{ }^{2} \cdot \mathrm{yr}\right)\end{array}$ & $\begin{array}{c}\text { Motors } \\
\left(\mathrm{kBtu} / \mathrm{ft}^{2} \cdot \mathrm{yr}\right)\end{array}$ & $\begin{array}{l}\text { Plug and } \\
\text { Process } \\
\text { Subtotal } \\
\left(\text { kBtu/ft }^{2} \cdot \mathrm{yr}\right)\end{array}$ & $\begin{array}{c}\text { Refrigeration } \\
\left(\mathrm{kBtu}^{2} / \mathrm{ft}^{2} \cdot \mathrm{yr}\right)\end{array}$ \\
\hline Small office & 7.47 & 2.66 & 0.34 & 0.34 & 0.00 & 0.75 & 11.57 & 1.98 \\
\hline Large office & 12.22 & 1.98 & 0.31 & 0.41 & 0.10 & 2.46 & 17.47 & 1.40 \\
\hline Restaurant & 2.15 & 3.86 & 0.07 & 35.42 & 0.03 & 0.92 & 42.45 & 33.68 \\
\hline $\begin{array}{l}\text { Refrigerated } \\
\text { warehouse }\end{array}$ & 0.58 & 1.94 & 0.78 & 0.14 & 0.14 & 6.21 & 9.79 & 45.86 \\
\hline $\begin{array}{l}\text { Nonrefrigerated } \\
\text { warehouse }\end{array}$ & 0.82 & 1.33 & 0.20 & 0.07 & 0.07 & 0.99 & 3.48 & 0.96 \\
\hline School & 1.57 & 0.85 & 0.03 & 0.61 & 0.00 & 0.27 & 3.34 & 1.71 \\
\hline College & 2.46 & 1.67 & 0.48 & 0.92 & 0.03 & 1.98 & 7.54 & 1.57 \\
\hline All warehouses & 0.78 & 1.43 & 0.27 & 0.07 & 0.07 & 1.77 & 4.40 & 7.54 \\
\hline
\end{tabular}


Table 2-2 CEUS Results for Plug and Process and Refrigeration Electricity Use Intensities: SI Units

\begin{tabular}{|c|c|c|c|c|c|c|c|c|}
\hline $\begin{array}{c}\text { CEUS Building } \\
\text { Type }\end{array}$ & $\begin{array}{c}\text { Office } \\
\text { Equipment } \\
\left(\mathrm{MJ} / \mathrm{m}^{2} \cdot \mathrm{yr}\right)\end{array}$ & $\begin{array}{l}\text { Miscellaneous } \\
\left(\mathrm{MJ} / \mathrm{m}^{2} \cdot \mathrm{yr}\right)\end{array}$ & $\begin{array}{l}\text { Process } \\
\left(\mathrm{MJ} / \mathrm{m}^{2} \cdot \mathrm{yr}\right)\end{array}$ & $\begin{array}{l}\text { Cooking } \\
\left(\mathrm{MJ} / \mathrm{m}^{2} \cdot \mathrm{yr}\right)\end{array}$ & $\begin{array}{c}\text { Air } \\
\text { Compressor } \\
\left(\mathrm{MJ} / \mathrm{m}^{2} \cdot \mathrm{yr}\right)\end{array}$ & $\begin{array}{c}\text { Motors } \\
\left(\mathrm{MJ} / \mathrm{m}^{2} \cdot \mathrm{yr}\right)\end{array}$ & $\begin{array}{l}\text { Plug and } \\
\text { Process } \\
\text { Subtotal } \\
\left(\mathrm{MJ} / \mathrm{m}^{2} \cdot \mathrm{yr}\right)\end{array}$ & $\begin{array}{c}\text { Refrigeration } \\
\left(\mathrm{MJ} / \mathrm{m}^{2} \cdot \mathrm{yr}\right)\end{array}$ \\
\hline All & 37.6 & 31.0 & 5.0 & 22.0 & 1.6 & 22.0 & 119.4 & 70.9 \\
\hline Small office & 84.9 & 30.2 & 3.9 & 3.9 & 0.0 & 8.5 & 131.5 & 22.5 \\
\hline Large office & 138.9 & 22.5 & 3.5 & 4.7 & 1.1 & 28.0 & 198.5 & 15.9 \\
\hline Restaurant & 24.4 & 43.9 & 0.8 & 402.5 & 0.3 & 10.5 & 482.4 & 382.7 \\
\hline Retail & 19.0 & 26.7 & 3.5 & 8.5 & 1.9 & 11.3 & 70.9 & 39.9 \\
\hline Food store & 14.3 & 36.8 & 1.6 & 71.7 & 0.3 & 6.9 & 131.8 & 869.3 \\
\hline $\begin{array}{l}\text { Refrigerated } \\
\text { warehouse }\end{array}$ & 6.6 & 22.0 & 8.9 & 1.6 & 1.6 & 70.6 & 111.3 & 521.1 \\
\hline $\begin{array}{l}\text { Nonrefrigerated } \\
\text { warehouse }\end{array}$ & 9.3 & 15.1 & 2.3 & 0.8 & 0.8 & 11.3 & 39.5 & 10.9 \\
\hline School & 17.8 & 9.7 & 0.3 & 6.9 & 0.0 & 3.1 & 38.0 & 19.4 \\
\hline College & 28.0 & 19.0 & 5.5 & 10.5 & 0.3 & 22.5 & 85.7 & 17.8 \\
\hline Health care & 33.3 & 97.7 & 8.5 & 16.7 & 0.3 & 30.2 & 186.9 & 27.5 \\
\hline Lodging & 6.6 & 43.1 & 0.8 & 26.4 & 0.0 & 18.6 & 95.3 & 34.9 \\
\hline Miscellaneous & 13.5 & 38.8 & 11.6 & 10.1 & 4.7 & 41.9 & 120.6 & 33.3 \\
\hline All offices & 119.8 & 25.2 & 3.5 & 4.3 & 0.8 & 20.9 & 174.4 & 18.2 \\
\hline All warehouses & 8.9 & 16.3 & 3.1 & 0.8 & 0.8 & 20.1 & 50.0 & 85.7 \\
\hline
\end{tabular}


Table 2-3 CEUS Results for Gas End Use Intensities: IP Units

\begin{tabular}{|c|c|c|c|c|}
\hline CEUS Building Type & $\begin{array}{c}\text { Cooking } \\
\left(\mathrm{kBtu} / \mathrm{ft}^{2} \cdot \mathrm{yr}\right)\end{array}$ & $\begin{array}{l}\text { Miscellaneous } \\
\left(\mathrm{kBtu} / \mathrm{ft}^{2} \cdot \mathrm{yr}\right)\end{array}$ & $\begin{array}{c}\text { Process } \\
\left(\mathrm{kBtu}^{2} / \mathrm{ft}^{2} \cdot \mathrm{yr}\right)\end{array}$ & 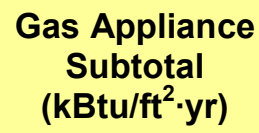 \\
\hline All & 5.9 & 0.5 & 1.5 & 7.9 \\
\hline Small office & 0.1 & 0.0 & 0.1 & 0.2 \\
\hline Large office & 0.2 & 0.1 & 1.2 & 1.5 \\
\hline Restaurant & 153.3 & 0.0 & 0.3 & 153.6 \\
\hline Retail & 0.5 & 0.3 & 0.0 & 0.8 \\
\hline Food store & 10.3 & 0.0 & 0.1 & 10.4 \\
\hline Refrigerated warehouse & 1.2 & 0.0 & 2.8 & 4.0 \\
\hline Nonrefrigerated warehouse & 0.0 & 0.0 & 0.0 & 0.0 \\
\hline School & 1.1 & 0.0 & 0.1 & 1.2 \\
\hline College & 1.7 & 0.9 & 0.0 & 2.6 \\
\hline Health care & 3.4 & 1.4 & 5.1 & 9.9 \\
\hline Lodging & 4.4 & 1.4 & 0.3 & 6.1 \\
\hline Miscellaneous & 1.0 & 1.0 & 4.6 & 6.6 \\
\hline All offices & 0.2 & 0.1 & 0.8 & 1.1 \\
\hline All warehouses & 0.2 & 0.0 & 0.4 & 0.6 \\
\hline
\end{tabular}

Table 2-4 CEUS Results for Gas End Use Intensities: SI Units

\begin{tabular}{|c|c|c|c|c|}
\hline CEUS Building Type & $\begin{array}{l}\text { Cooking } \\
\left(\mathrm{MJ} / \mathrm{m}^{2} \cdot \mathrm{yr}\right)\end{array}$ & $\begin{array}{l}\text { Miscellaneous } \\
\left(\mathrm{MJ} / \mathrm{m}^{2} \cdot \mathrm{yr}\right)\end{array}$ & $\begin{array}{l}\text { Process } \\
\left(\mathrm{MJ} / \mathrm{m}^{2} \cdot \mathrm{yr}\right)\end{array}$ & $\begin{array}{c}\text { Gas Appliance } \\
\text { Subtotal } \\
\left(\mathrm{MJ} / \mathrm{m}^{2} \cdot \mathrm{yr}\right)\end{array}$ \\
\hline All & 67 & 6 & 17 & 90 \\
\hline Small office & 1 & 0 & 1 & 2 \\
\hline Large office & 2 & 1 & 14 & 17 \\
\hline Restaurant & 1,742 & 0 & 3 & 1,745 \\
\hline Retail & 6 & 3 & 0 & 9 \\
\hline Food store & 117 & 0 & 1 & 118 \\
\hline Refrigerated warehouse & 14 & 0 & 32 & 45 \\
\hline Nonrefrigerated warehouse & 0 & 0 & 0 & 0 \\
\hline School & 13 & 0 & 1 & 14 \\
\hline College & 19 & 10 & 0 & 30 \\
\hline Health & 39 & 16 & 58 & 113 \\
\hline Lodging & 50 & 16 & 3 & 69 \\
\hline Miscellaneous & 11 & 11 & 52 & 75 \\
\hline All offices & 2 & 1 & 9 & 13 \\
\hline All warehouses & 2 & 0 & 5 & 7 \\
\hline
\end{tabular}




\subsection{Lighting}

DOE (2002) commissioned Navigant Consulting, Inc., to characterize the U.S. lighting market. That study used data from 1999 CBECS (EIA 2002) and the XenCap ${ }^{\mathrm{TM}}$ energy auditing system to characterize lighting energy in the commercial sector. We assume (out of practical necessity) that Navigant's results still apply when moving from 1999 CBECS to 2003 CBECS. Table 2-5 lists data (from Table 5-11 in DOE 2002) used to develop inputs for lighting installed density in the current methodology. (The energy use intensity for vacant buildings seem to be in error.)

Table 2-5 U.S. Lighting Market Characterization Results for Lighting Electricity Use

\begin{tabular}{|c|c|c|c|c|}
\hline Subsector (PBA) & $\begin{array}{l}\text { Lighting Power } \\
\text { Density } \\
\left(\mathrm{W} / \mathrm{ft}^{2}\right)\end{array}$ & $\begin{array}{l}\text { Lighting Power } \\
\text { Density } \\
\left(\mathrm{W} / \mathrm{m}^{2}\right)\end{array}$ & $\begin{array}{c}\text { Lighting } \\
\text { Electricity Use } \\
\text { Intensity } \\
\left(\mathbf{k B t u} / \mathrm{ft}^{2} \cdot \mathbf{y r}\right)\end{array}$ & $\begin{array}{l}\text { Lighting } \\
\text { Electricity Use } \\
\text { Intensity } \\
\left(\mathrm{MJ} / \mathrm{m}^{2} \cdot \mathrm{yr}\right)\end{array}$ \\
\hline בOffice/professional & 1.8 & 19.4 & 22.9 & 260 \\
\hline $\begin{array}{l}\text { Nonrefrigerated } \\
\text { warehouse }\end{array}$ & 1.4 & 15.1 & 16.4 & 186 \\
\hline Education & 1.8 & 19.4 & 16.7 & 190 \\
\hline Retail (except malls) & 1.9 & 20.5 & 23.9 & 272 \\
\hline Public assembly & 1.4 & 15.1 & 12.3 & 140 \\
\hline Service & 1.7 & 18.3 & 19.4 & 220 \\
\hline Religious worship & 1.4 & 15.1 & 8.5 & 97 \\
\hline Lodging & 1.3 & 14.0 & 16.0 & 182 \\
\hline Food services & 1.6 & 17.2 & 23.9 & 272 \\
\hline Inpatient health care & 1.7 & 18.3 & 32.4 & 368 \\
\hline Public order and safety & 1.3 & 14.0 & 15.4 & 175 \\
\hline Food store & 1.9 & 20.5 & 33.4 & 380 \\
\hline Outpatient health care & 1.7 & 18.3 & 19.8 & 225 \\
\hline Vacant & 2.1 & 22.6 & 25.6 & 291 \\
\hline Other & 1.7 & 18.3 & 20.8 & 236 \\
\hline Skilled nursing & 1.3 & 14.0 & 18.4 & 209 \\
\hline Laboratory & 1.7 & 18.3 & 28.3 & 322 \\
\hline Refrigerated warehouse & 1.4 & 15.1 & 18.1 & 206 \\
\hline
\end{tabular}

\section{$2.4 \quad$ Envelope}

Huang and Franconi (1999) defined performance levels for opaque envelopes as part of their study on component loads. We have used their R-factor assignments as reorganized and presented in Table 2-6 for walls and Table 2-7 for roofs. They divided the United States into two geographic regions, north and south, based on degree days. 
Table 2-6 Wall R-Factor Assignments by Building Type, Activity, and Geography (Huang and Franconi 1999)

\begin{tabular}{|c|c|c|c|c|}
\hline \multirow[b]{2}{*}{ Building Type } & \multicolumn{2}{|c|}{ Old Vintage (pre-1980) } & \multicolumn{2}{|c|}{ New Vintage (post-1980) } \\
\hline & $\begin{array}{c}\text { North } \\
\text { Btu/h.ft' } \\
\left(\mathrm{W} / \mathrm{m}^{2} \cdot \mathrm{K}\right)\end{array}$ & $\begin{array}{c}\text { South } \\
\text { Btu/h'ft' } \mathrm{ft}^{2} \cdot \mathrm{F} \\
\left(\mathrm{W} / \mathrm{m}^{2} \cdot \mathrm{K}\right)\end{array}$ & $\begin{array}{c}\text { North } \\
\text { Btu/h' } \cdot \mathrm{ft}^{2} \cdot \mathrm{F} \\
\left(\mathrm{W} / \mathrm{m}^{2} \cdot \mathrm{K}\right)\end{array}$ & 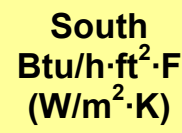 \\
\hline Small office & $4.9(1.158)$ & $3.9(1.456)$ & $6.3(0.901)$ & $5.6(1.014)$ \\
\hline Large office & $2.5(2.271)$ & $2.5(2.271)$ & $4.6(1.234)$ & $6.0(0.946)$ \\
\hline Small retail & $3.4(1.67)$ & $2.5(2.271)$ & $6.6(0.86)$ & $4.8(1.183)$ \\
\hline Large retail & $3.1(1.832)$ & $3.3(1.721)$ & $6.4(0.887)$ & $4.8(1.183)$ \\
\hline Small hotel & $3.4(1.67)$ & $3.4(1.67)$ & $5.3(1.071)$ & $5.3(1.071)$ \\
\hline Large hotel & $3.6(1.577)$ & $3.6(1.577)$ & $6.2(0.916)$ & $6.2(0.916)$ \\
\hline Fast food restaurant & $10.9(0.521)$ & $10.9(0.521)$ & $13.2(0.43)$ & $13.2(0.43)$ \\
\hline Sit down restaurant & $10.9(0.521)$ & $10.9(0.521)$ & $13.2(0.43)$ & $13.2(0.43)$ \\
\hline Hospital & $4.3(1.321)$ & $4.3(1.321)$ & $6.9(0.823)$ & $6.9(0.823)$ \\
\hline School & $2.7(2.103)$ & $3.4(1.67)$ & $5.3(1.07)$ & $5.7(0.996)$ \\
\hline Supermarket & $3.3(1.721)$ & $3.3(1.721)$ & $5.8(0.979)$ & $5.8(0.979)$ \\
\hline Warehouse & $3.2(1.774)$ & $2.4(2.366)$ & $4.6(1.234)$ & $4.0(1.42)$ \\
\hline
\end{tabular}

Table 2-7 Roof R-Factor Assignments by Building Type, Activity, and Geography (Huang and Franconi 1999)

\begin{tabular}{|c|c|c|c|c|}
\hline \multirow[t]{2}{*}{ Building Type } & \multicolumn{2}{|c|}{ Old Vintage (pre-1980) } & \multicolumn{2}{|c|}{ New Vintage (post-1980) } \\
\hline & $\begin{array}{c}\text { North } \\
\text { Btu/h'ft' }{ }^{2} \cdot F \\
\left(\mathrm{~W} / \mathrm{m}^{2} \cdot \mathrm{K}\right)\end{array}$ & $\begin{array}{c}\text { South } \\
\text { Btu/h: } \mathrm{ft}^{2} \cdot \mathrm{F} \\
\left(\mathrm{W} / \mathrm{m}^{2} \cdot \mathrm{K}\right)\end{array}$ & $\begin{array}{c}\text { North } \\
\text { Btu/h' } \mathrm{ft}^{2} \cdot \mathrm{F} \\
\left(\mathrm{W} / \mathrm{m}^{2} \cdot \mathrm{K}\right)\end{array}$ & $\begin{array}{c}\text { South } \\
\text { Btu/h } \mathrm{ft}^{2} \cdot F \\
\left(\mathrm{~W} / \mathrm{m}^{2} \cdot \mathrm{K}\right)\end{array}$ \\
\hline Small office & $11.9(0.477)$ & $10.5(0.541)$ & $13.3(0.427)$ & $12.6(0.451)$ \\
\hline Large office & $9.1(0.624)$ & $11.2(0.507)$ & $9.1(0.624)$ & $12.6(0.451)$ \\
\hline Small retail & $10.2(0.557)$ & $9.5(0.598)$ & $13.2(0.43)$ & $12.0(0.473)$ \\
\hline Large retail & $10.6(0.536)$ & $11.5(0.494)$ & $14.0(0.406)$ & $12.0(0.473)$ \\
\hline Small hotel & $9.8(0.579)$ & $9.8(0.579)$ & $13.2(0.43)$ & $13.2(0.43)$ \\
\hline Large hotel & $11.8(0.481)$ & $11.8(0.481)$ & $14.0(0.406)$ & $14.0(0.406)$ \\
\hline Fast food restaurant & $10.9(0.521)$ & $10.9(0.521)$ & $13.2(0.43)$ & $13.2(0.43)$ \\
\hline Sit down restaurant & $10.9(0.521)$ & $10.9(0.521)$ & $13.2(0.43)$ & $13.2(0.43)$ \\
\hline Hospital $^{*}$ & $12.3(0.462)$ & $12.3(0.462)$ & $11.5(0.494)$ & $11.5(0.494)$ \\
\hline School & $10.9(0.521)$ & $10.1(0.562)$ & $12.6(0.451)$ & $13.3(0.427)$ \\
\hline Supermarket & $9.2(0.617)$ & $9.2(0.617)$ & $11.5(0.494)$ & $11.5(0.494)$ \\
\hline Warehouse & $7.8(0.728)$ & $7.6(0.747)$ & $10.1(0.562)$ & $10.6(0.536)$ \\
\hline
\end{tabular}

*The roof insulation decreased for new hospitals.

\subsection{Outside Air}

Outside air is introduced in buildings intentionally as mechanical ventilation and unintentionally as infiltration. Additional research is needed to fully characterize outside air rates across the commercial sector; however, there have been some efforts in the literature to do so. Most such efforts combine mechanical ventilation and infiltration. Orme (2001) estimated the energy impact of controlled (mechanical) and uncontrolled (infiltration) ventilation by assuming air change rates of 0.75 air changes per hour (ACH) for all commercial (service sector) buildings in all climates and all countries. Colliver 
(1995) modeled the specific energy requirements of ventilation air per kilogram of ventilation air, but did not extend analyses to include the amount or rates of ventilation air. Fisk (2000) summarized studies of sick building syndrome that reported ventilation rates varying from 0 to 60 liters per second per person (1/s-person), but there is no indication that these ventilation rates are representative of the stock. Seppanen et al. (1999) characterize the normal range of ventilation at 5 to $30 \mathrm{l} / \mathrm{s}$-person.

Chan (2006) reviewed the literature with measured data and modeled air leakage from commercial buildings. He used data about the shapes of commercial buildings in Oklahoma City to arrive at a distribution for the infiltration rates in commercial buildings. Chan's modeling was based on 1999 CBECS; he found that infiltration rates in the commercial sector are distributed according to a probability density function (PDF) defined by a lognormal distribution with a geometric mean of $0.35 \mathrm{ACH}$ and a geometric standard deviation of 2.1 ACH (see Figure 5-22 in Chan 2006). Chan's distribution was selected for use in assigning infiltration rates in the current methodology.

Persily (1998) reviewed literature with measured data about airtightness from a combined total of 139 commercial buildings. As Persily points out, "There is no simple calculation method or rule of thumb to relate envelope tightness to infiltration in commercial buildings..." Because leakiness is evaluated at elevated pressure differences, translating these rates into infiltration rates for use in annual energy modeling is difficult without using detailed airflow network models. Persily found a mean infiltration rate of $27.1 \mathrm{~m}^{3} / \mathrm{h} \cdot \mathrm{m}^{2}\left(1.5 \mathrm{cfm} / \mathrm{ft}^{2}\right)$ at $75 \mathrm{~Pa}(0.3 \mathrm{in}$. of water $)$ and concluded that commercial buildings are not tighter than residential buildings. Although the data set is minimal and not formulated from a suitable random sample, he also concluded that there is little evidence to support the notion that age and type of construction affect airtightness.

Turk et al. (1989) used tracer gas techniques to measure ventilation rates in 38 commercial buildings in the Pacific Northwest. Table 2-8 lists the results used in the current study. They found mean ventilation rates of $1.5 \mathrm{ACH}$ or $28 \mathrm{l} / \mathrm{s}$-person and provided a breakdown for five building classifications:

educational, libraries, small and large offices, and multi-use. These measured outside air rates are roughly twice those assumed by Orme. These data are based on a relatively small sample size with limited types of buildings and geographic regions, and therefore provide only a rough estimate of actual outside air rates across the sector. However, they appear to be as good as anything available; we adapted them to develop inputs for mechanical ventilation.

Table 2-8 Measured Outside Air Rates (Turk et al. 1989)

\begin{tabular}{|l|c|c|c|c|}
\hline \multicolumn{1}{|c|}{ Building Classification } & $\mathbf{N}$ & $\begin{array}{c}\text { Outside Air Rate } \\
\text { (ACH) }\end{array}$ & $\begin{array}{c}\text { Outside Air Rate } \\
\text { (cfm/person) }\end{array}$ & $\begin{array}{c}\text { Outside Air Rate } \\
\text { (I/s·person) }\end{array}$ \\
\hline \hline Educational & 7 & 1.9 & 33 & 15.6 \\
\hline $\begin{array}{l}\text { Office/professional } \\
<100,000 \mathrm{ft}^{2}\left(9,300 \mathrm{~m}^{2}\right)\end{array}$ & 8 & 1.5 & 75 & 35.4 \\
\hline $\begin{array}{l}\text { Office/professional } \\
>100,000 \mathrm{ft}^{2}\left(9,300 \mathrm{~m}^{2}\right)\end{array}$ & 14 & 1.8 & 65 & 30.7 \\
\hline Libraries & 3 & 0.6 & 71 & 33.5 \\
\hline Multi-use & 5 & 1.4 & 60 & 28.3 \\
\hline Naturally ventilated & 3 & 0.8 & 38 & 17.9 \\
\hline
\end{tabular}




\section{Methodology}

This section describes the methodology developed to model building energy performance across the entire commercial sector. The basic framework for the analysis is diagrammed in Figure 3-1. This report covers part of a larger study conducted to assess the commercial sector. The second and third parts of the study are included in two separate reports that are closely related to this one. This first report documents the methods used in the second report to analyze the technical potential for net-zero energy buildings (Griffith et al. 2007) and in the third report to explore the energy impacts of outside air (Benne et al. 2008).

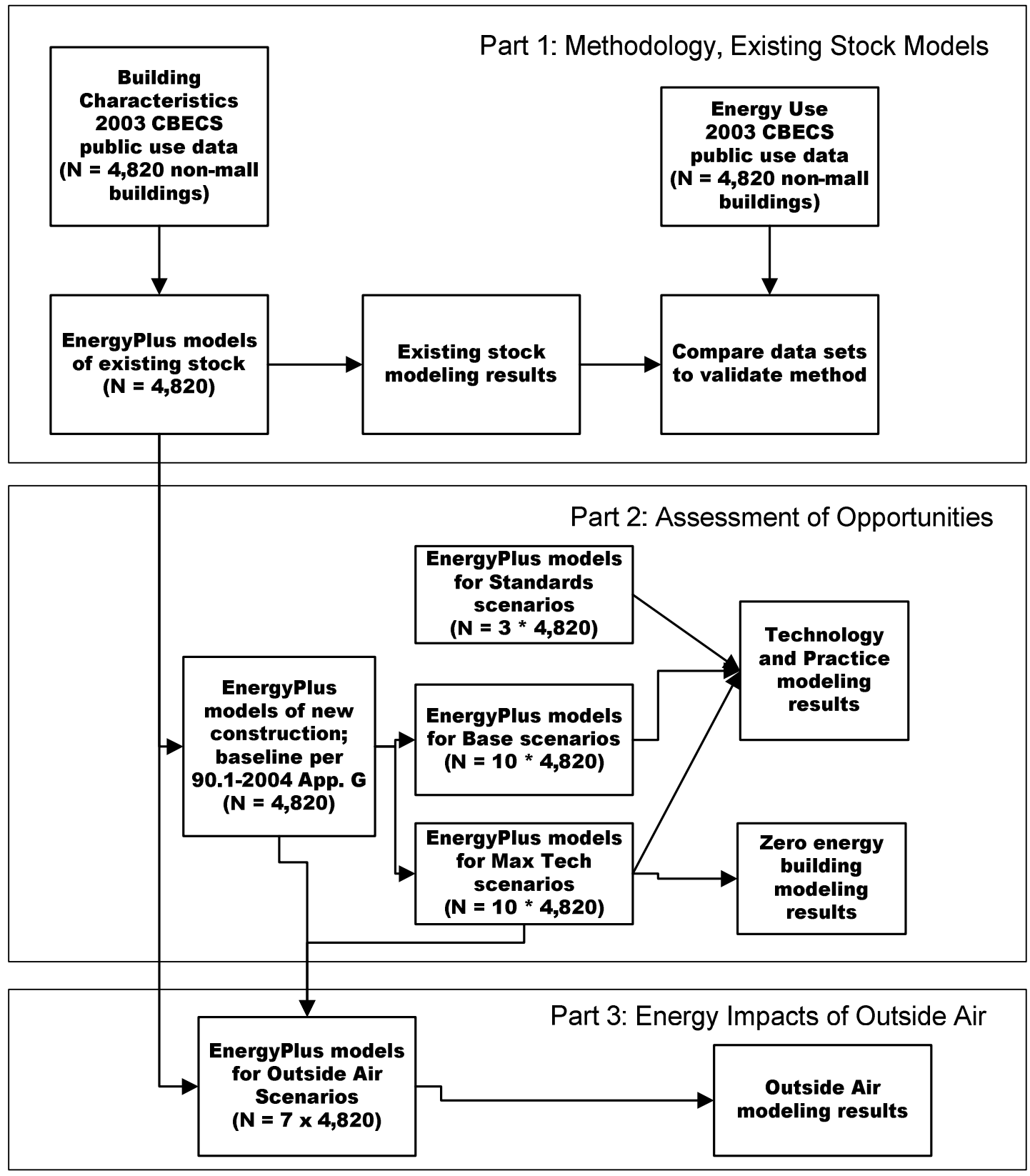

Figure 3-1 Overview of Commercial Sector Assessment 


\subsection{Background}

Whole-building simulation has been in use for approximately 30 years, and researchers have long used such tools to represent large portions of the building stock. Some of the earliest research was conducted at Pacific Northwest National Laboratory (Briggs, Crawley, and Belzer 1987; Briggs, Crawley, and Schliesing 1992; Crawley and Schliesing 1992). A more current example is research by Huang and Franconi (1999) at Lawrence Berkeley National Laboratory (LBNL), who built on this work. The LBNL researchers focused on modeling component loads for the building stock. Moffat (2001) presents a good overview of these methods, which he refers to as stock aggregation, in the context of life-cycle analysis and community planning. Large-scale simulation studies are also common in the history of developing codes and standards. The earlier projects demonstrated the utility of running large numbers of detailed models to address certain questions.

We selected EnergyPlus (DOE 2007) because it is the contemporary DOE/BT energy simulation tool; it accounts for the complex interactions between climate, internal gains, building form and fabric, HVAC systems, and renewable energy systems. The simulations are run with a custom version of EnergyPlus Version 2.0 compiled to run on a 64-bit cluster computer at NREL. EnergyPlus is a heavily tested program; formal BESTEST validation efforts were repeated for every release (Judkoff and Neymark 1995; ASHRAE 2004b).

Defining a building model for the EnergyPlus simulation program requires considerable detail. Table 3-1 lists examples of input parameters we use to structure these details into the following four groups:

- Program refers to the architectural program, which describes how the building will be used and the services it must deliver to the occupants. From an energy point of view, program decisions influence many important drivers (climate, plug and process loads, ventilation requirements, operating schedules, and comfort tolerances) that will ultimately determine energy performance.

- Form refers to the geometry of the building and its elements, and has important energy implications that stem from how the building interacts with the sun and ambient conditions.

- Fabric refers to the materials used to construct the building and involves decisions about insulation levels, glazing systems, and thermal mass.

- Equipment includes HVAC equipment, lighting systems and controls. Except for plug and process load equipment selected by the occupants, this includes all the energy-consuming equipment that is part of the building.

Table 3-1 Model Parameter Categories with Sample Parameters

\begin{tabular}{|l|l|l|l|}
\hline \multicolumn{1}{|c|}{ Program } & \multicolumn{1}{c|}{ Form } & \multicolumn{1}{c|}{ Fabric } & \multicolumn{1}{c|}{ Equipment } \\
\hline \hline Facility location & Floor plate & Exterior walls & HVAC system types \\
Total floor area & Number of floors & Roof & Component efficiency \\
Schedules & Aspect ratio & Windows & Control settings \\
Plug and process loads & Window fraction & Interior partitions & Lighting fixtures \\
Lighting levels & Window locations & Internal mass & Lamp types \\
Ventilation needs & Shading & & Daylighting controls \\
Occupancy & Floor height & & \\
Site constraints & Orientation & & \\
\hline
\end{tabular}

\subsection{Model Generation}

The process used in the methodology is to take each building in the 2003 CBECS public use data files and create an EnergyPlus model that approximates it. A statistical model of the commercial buildings energy sector is formed by the survey's sample buildings, which have weighting factors that indicate how many more such buildings are represented by each sample. Energy consumption data for malls are available from 2003 CBECS, but malls were excluded from the study because their characteristics are not described 
well in the 2003 CBECS public use data (to preserve anonymity). For each building, we used a variety of CBECS data about floor area, number of floors, census division, basic climatic design criteria, PBA, number of employees, operating hours, type of heating and cooling system, type of windows, and many other variables. However, EnergyPlus models require much more detail about buildings than is available in the 2003 CBECS data. Therefore, an important part of the methodology is to synthesize the additional detail needed in the EnergyPlus input file. This is accomplished by complementing the 2003 CBECS data set with a mixture of literature sources, probabilistic assignments, and assumptions. The details of these translations are lengthy and are only summarized here; more thorough documentation is found in Appendix C.

The process of developing the method included some iteration to target data sources. In most cases, using inverse methods to back out the input that will produce a desired result is too difficult. In targeting occupant-driven plug and process load data from CEUS, the iteration method is used to adjust certain coefficients in the input generating routines. Only energy consumption data were used. All iterations involved rerunning the entire set of models, typically incorporating bug fixes in routines or EnergyPlus routines, or both, and adjusted input modeling coefficients.

\subsubsection{Program}

The first step in developing a building model is to define the architectural program for the building being evaluated. The type of activity a building is used for is provided directly by CBECS data for PBA and "more specific building activity" (PBAPLUS). Both classification systems were used to generate a variety of model inputs. Locations for all buildings were modeled from CBECS data by finding the weather file location that most closely matched CBECS data for census division and degree days (see Section C.2). These modeled locations are then used to determine a number of model inputs, including climatic design conditions for sizing, ground temperatures, utility tariffs, and source, emission, and water energy factors, that have geographic variation. The total floor area of the building is provided by CBECS. Operating schedules were modeled from a variety of CBECS data for the number of operating hours per week, whether the business is open on weekends, and other factors (see Section C.5). Occupant-driven plug and process loads are modeled from building activity and use data discussed in Section 2 (see

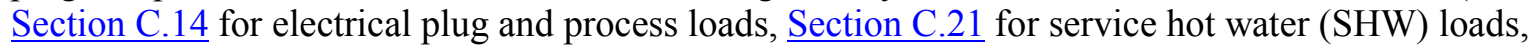

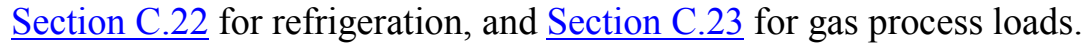

\subsubsection{Form}

To develop the EnergyPlus models efficiently, we assumed that building floor plates were rectangular, above grade, and uniform from floor to floor. The thermal models use a five-zone per floor zoning pattern. In reality, five thermal zones are too many for buildings with smaller floor plates and too few for large complex buildings, so this is a compromise that can be applied to all buildings. The number of floors is provided directly by CBECS data. The overall shape of the rectangular buildings is described by an aspect ratio, which is the east-west length divided by the north-south length. Probabilistic assignments were used to assign aspect ratios and orientations of the buildings (see Section C.7). The exterior windows were modeled from CBECS data for exterior glazing area with probabilistic assignments, and the locations and shapes of the windows were assumed (see Section C.11). Floor-tofloor heights used probabilistic assignments that varied by PBA (see Section C.9).

\subsubsection{Fabric}

The materials and constructions used for each building were modeled from CBECS data (see Section C.12). The composition of exterior walls and roofs was developed from CBECS data about the types of construction and R-factor data from Huang and Franconi (1999). The composition of window glazings was modeled from CBECS data about window types and tints or reflective coatings. The compositions of interior partitions and internal mass were assumed. 
We used literature data and a combination of a flow per unit area of exterior surfaces and a low-level whole-zone air change rate to model the infiltration behavior of the exterior envelope (see Section C.16).

\subsubsection{Equipment}

Electric lighting power density (LPD) was set probabilistically by using mean values from literature and assumed standard deviations that varied by PBA (see Section C.15). CBECS data for the type of heating and cooling systems were used to assign HVAC system types. Buildings were assumed to be either entirely conditioned or not conditioned at all; a 25\% threshold for the percent conditioned data from CBECS was used to make the determination separately for heating and cooling. Fifty-two HVAC topologies were used in the modeling (See Section C.18). The performance characteristics of the primary components in the HVAC system were modeled from the age of the building by using assumed efficiencies developed from different vintages of building energy standards. A linear function of estimated age of equipment was used to model equipment degradation (see Section C.19). Thermostatic control settings were incorporated into schedules that follow the operating hours and typical setup and setback practices (see Section C.5, Table C-16). Mechanical ventilation was modeled from literature data (see Section C.20).

\subsection{Validation}

This section describes the process used to validate the methodology. The validity of the method needs to be examined before it is used in studies that address energy performance across the commercial sector.

The overall process used to validate the bottom-up modeling method is to run the models in EnergyPlus and then compare the modeling results to the CBECS survey results. The model development was "blind" in that we did not use 2003 CBECS survey results for energy performance when we developed input data. Instead, we reserved the energy performance results for validation. Although no formal controls were put in place to guarantee "blindness" after the energy performance data were released, the methods for generating inputs were developed during a time when EIA had released only the preliminary data set for building characteristics. (EIA released 2003 CBECS public use data in multiple phases, and the first set did not include the energy consumption results.) However, model input was iterated to target literature data. The authors were experienced in the sector and therefore had general knowledge of typical performance and the results from 1999 CBECS. The process includes four steps:

1. Generate the EnergyPlus models. Computer routines were implemented that automate the creation of model input files, because manual methods would not be feasible for such a large number of building models. The approach documented in this report was used to generate each of the 4,820 models.

2. Simulate the buildings by running the models in EnergyPlus. Computer routines were implemented that manage the execution of each EnergyPlus model. For a fair comparison between the models and the survey, the weather data used in the modeling corresponded to the actual weather of the survey year. These 2003 historical weather files were produced in EnergyPlus format from data in the National Solar Radiation Database 1991-2005 Update: User Manual (NREL 2007).

3. Collect and analyze the energy performance results from the modeling. Computer routines were implemented that automate the process of extracting key data from the EnergyPlus output files and storing them in a database. Analysis routines were implemented that allow aggregating results from the different buildings to produce values such as weighted mean, weighted standard deviation, and PDFs for various metrics and output bases. NREL made a key assumption that the CBECS weighting factors were still applicable, although many details of the survey buildings were unknown and were generated synthetically.

4. Collect and analyze the energy performance results from 2003 CBECS public use data. Computer routines were implemented that allow aggregating the results from the survey in the 
same ways as the results from the modeling. The survey results were also normalized to calculate energy use intensity (EUI) so the performance can be compared across different sized buildings. Survey public use data from EIA does not directly include EUI or ASHRAE climate zone; values in this report survey EUI and climate were modeled from other survey results.

The validity of the method was then examined by comparing the results from the existing stock models to the survey results in an effort to explore the validity of the bottom-up method being used to model the sector. We used the following metrics for the comparisons (for more on metric definitions see Barley et al. [2005]):

- Total site EUI is the sum of all the energy used by the building normalized by the total floor area of the building. Electricity and gas are combined without regard for their production and delivery. This metric ignores any energy produced at the site.

- Electricity use intensity is the sum of all electricity used by the building, normalized by the total floor area of the building.

- Natural gas use intensity is the sum of all natural gas used by the building, normalized by the total floor area of the building.

- Energy cost intensity is the sum of the costs of all energy used by the building, normalized by the total floor area of the building. The costs are the results of detailed tariff modeling; realistic utility rates were calculated for each building model. The tariff structures vary by location and reflect the complexities of demand charges and time-of-use (TOU) rates. These data were compiled from Web sources (Tariff Analysis Project, utilities, and EIA) and made into EnergyPlus input objects.

For this study, the methodology is to be considered valid if the weighted mean results from the models agree with the survey to within:

- One weighted standard deviation in the survey results

- $15 \%$ absolute EUI

- $20 \%$ absolute electricity use intensity

- $20 \%$ absolute natural gas use intensity

- $15 \%$ absolute energy cost intensity. 


\section{Results and Discussion}

In this section we compare results from the modeling effort and 2003 CBECS. The comparisons are generally based on averages of large numbers of buildings, because individual samples vary too widely. The intensity metrics used for results are normalized by building floor area so different buildings can be combined in weighted averages. The populations of buildings were examined in different ways, including by subsector where buildings are grouped by PBA, by climate zone where buildings are grouped by climate zones assigned to samples in this study, and by census division. Individual subsections examine different metrics such as total energy use, electricity use, natural gas use, and total energy costs.

\subsection{Total Energy Use}

Figure 4-1 shows results by subsector for the weighted mean results for total site EUI. The numeric values are provided in Table A-4 along with the weighted standard deviation of the EUI values. Overall, the models agree to within $12 \%$. The modeling tends to track the survey results fairly well across different subsectors, except for education, food service, inpatient health, and public order and safety.

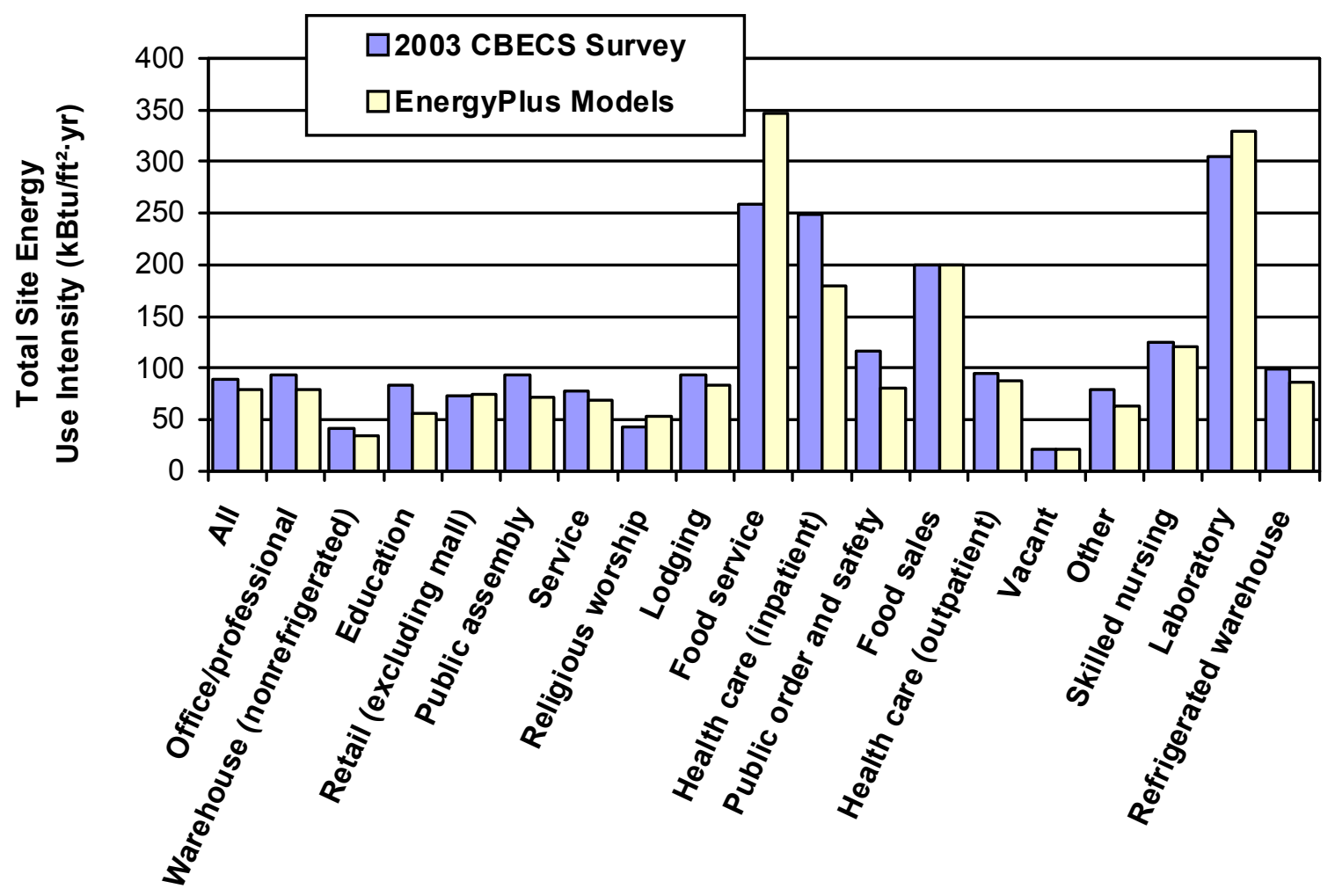

Subsector

Figure 4-1 Total EUI: 2003 CBECS Survey and Modeling by Subsector

Figure 4-2 shows results by climate zone for the weighted mean results for total site EUI. Table A-5 contains the values along with the weighted standard deviation in the EUI values and the number of buildings. The climate zone classification is defined in ANSI/ASHRAE Standard 169-2006 (ASHRAE 2006) and shown in Figure 4-3. The climate zone data here are extrapolated from 2003 CBECS and are not part of the CBECS public data set. NREL assigned a 169-2006 climate zone to each sample building by fitting survey data for heating degree days (HDDs) and cooling degree days (CDDs). We used degree days from 2003 historical weather data rather than typical or average conditions. The 2003 CBECS 
survey was not organized around these climate zones, and some of these classifications, including 1A, 2B, $3 \mathrm{C}, 4 \mathrm{~B}, 4 \mathrm{C}$, and 7 , have too few samples to be statistically valid. The main body of results here used historical weather data for 2003, but Figure 4-2 also shows modeling results with TMY data. The modeling results track survey results for climate zones $2 \mathrm{~A}, 3 \mathrm{~B}, 3 \mathrm{C}, 6 \mathrm{~A}, 6 \mathrm{~B}$, and $5 \mathrm{~B}$, but not $1 \mathrm{~A}, 4 \mathrm{~B}, 4 \mathrm{C}$, or 7A. The climate zones with the poorest agreement are those with the fewest buildings in the set.

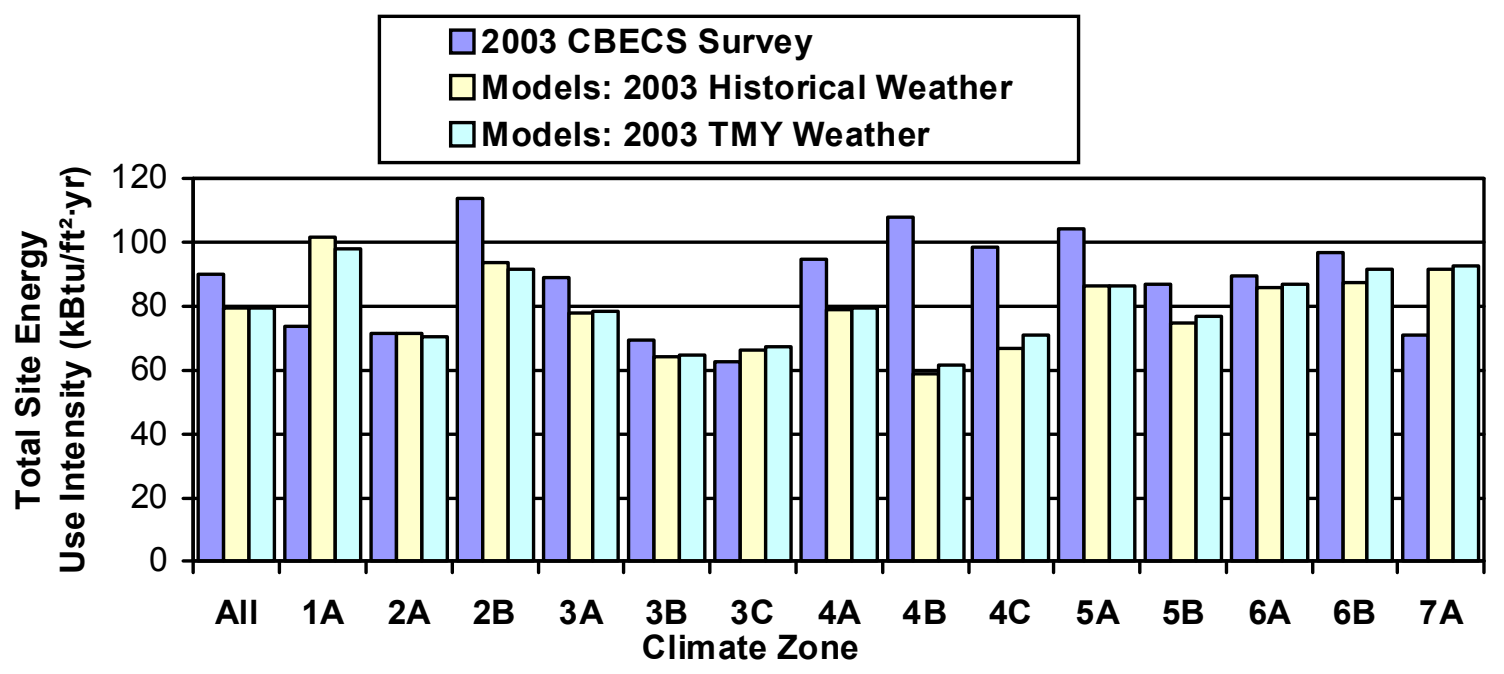

Figure 4-2 Total EUI: 2003 CBECS Survey and Modeling by Climate Zone

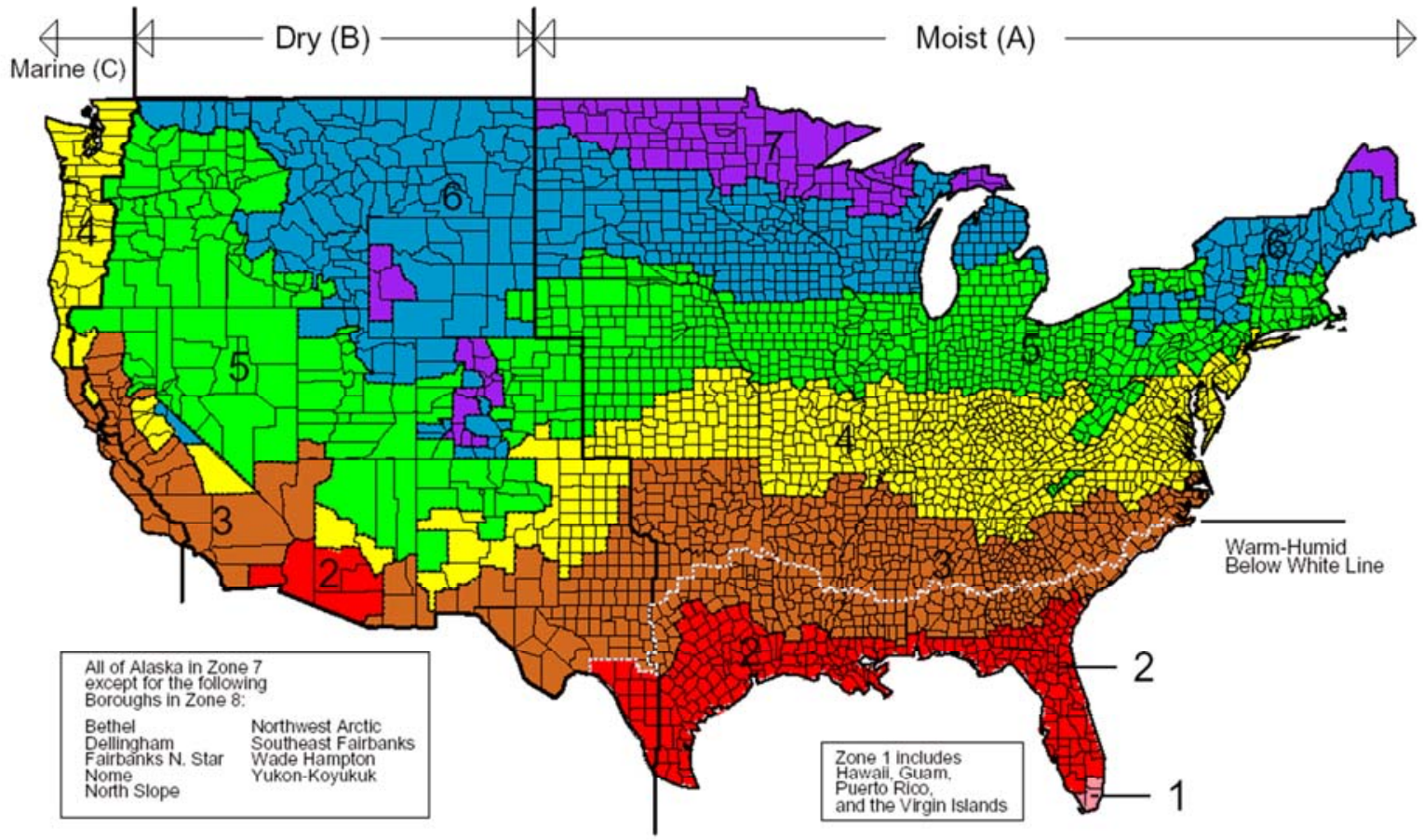

Figure 4-3 Map of ASHRAE 169-2006 Climate Zones

(Figure reprinted from

www.energycodes.gov/implement/pdfs/color map climate zones Mar03.pdf) 
Figure 4-4 shows results by census division for the weighted mean results for total site EUI. (Table A-6 has the values along with the weighted standard deviation in the EUI values and the number of buildings.) Census divisions are defined by the U.S. Department of Commerce. Relatively good agreement was obtained for Pacific, West South Central, New England, and South Atlantic census divisions. The two census divisions with relatively poor agreement are East North Central and Mountain.

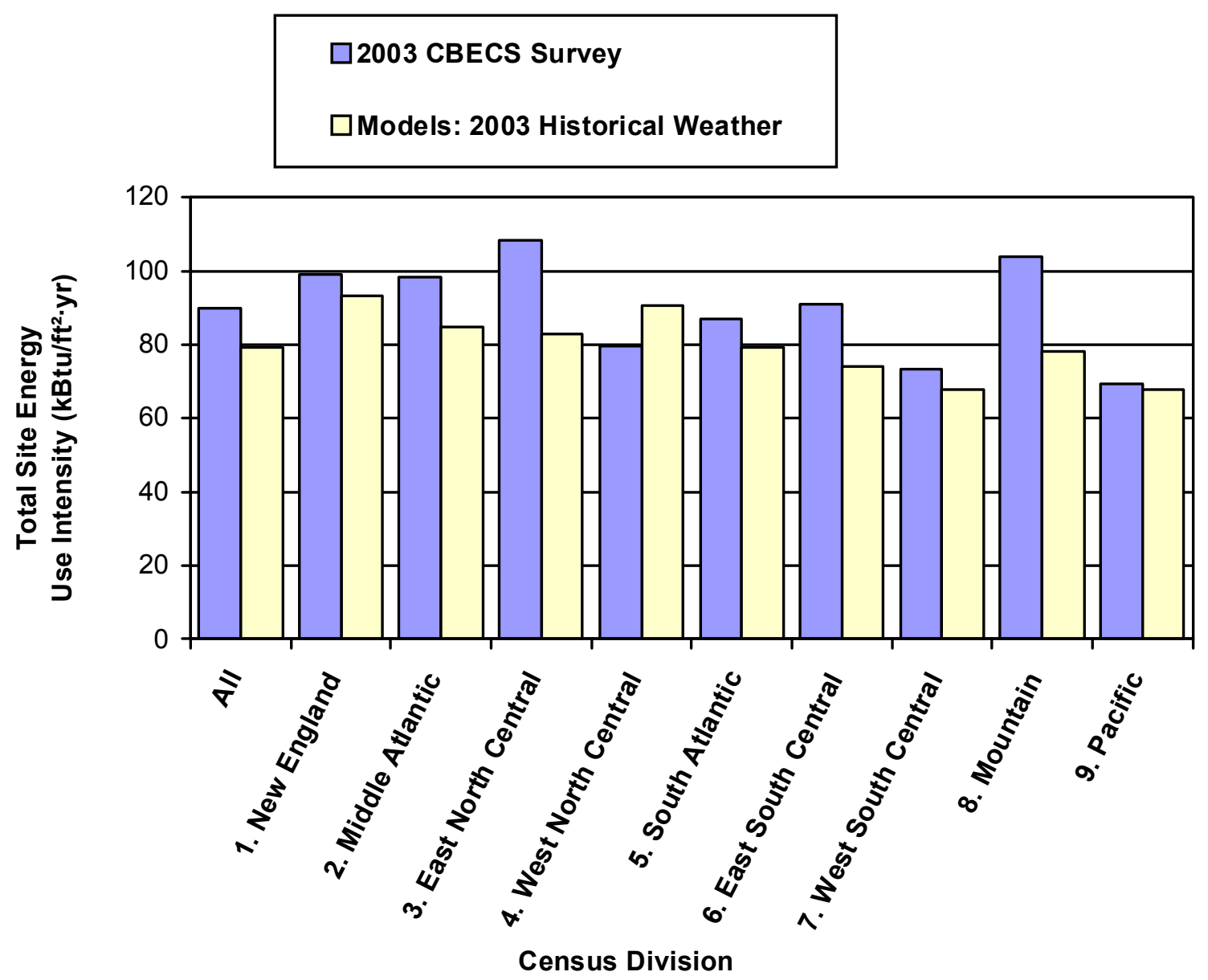

Figure 4-4 Total EUI: 2003 CBECS Survey and Modeling by Census Division

Mean values for net EUI values from 2003 CBECS are provided in Table 4-1 and Table 4-3 by subsector and climate zone. Table 4-2 and Table 4-4 list the net EUI values for the existing stock models. These tables also include values for the weighted standard deviation $(\sigma)$ in the results. The gray boxes indicate there are no models because the 2003 CBECS sample did not include any buildings in that particular category. The number of models and samples for each category are listed in Table 4-5. The comparison between models and survey requires a relatively large number of samples, but many categories do not have sufficient numbers. A specific criterion for how many samples are required for meaningful averages is difficult to determine. But because of the masking and shifting of floor area in CBECS public use data, the criterion for number of samples for area normalized intensity metrics is currently estimated at 100 . Table 4-6 lists the percentage differences in total EUI between the model and survey results. 
Table 4-1 2003 CBECS Weighted Mean EUI and Standard Deviation by Subsector and Climate Zone:

IP Units kBtu/ft ${ }^{2} \cdot \mathrm{yr}$

\begin{tabular}{|c|c|c|c|c|c|c|c|c|c|c|c|c|c|c|c|}
\hline \multirow[b]{2}{*}{ Subsector } & \multicolumn{15}{|c|}{ Climate Zone } \\
\hline & All & $1 \mathrm{~A}$ & $2 A$ & 2B & 3A & 3B & $3 C$ & 4A & 4B & 4C & $5 A$ & 5B & $6 \mathrm{~A}$ & 6B & 7 \\
\hline All & $\begin{array}{c}90 \\
\sigma=97\end{array}$ & $\begin{array}{c}74 \\
\sigma=98\end{array}$ & $\begin{array}{c}72 \\
\sigma=88\end{array}$ & $\begin{array}{c}114 \\
\sigma=92\end{array}$ & $\begin{array}{c}89 \\
\sigma=105\end{array}$ & $\begin{array}{c}70 \\
\sigma=98\end{array}$ & $\begin{array}{c}62 \\
\sigma=54\end{array}$ & $\begin{array}{c}95 \\
\sigma=105\end{array}$ & $\begin{array}{c}108 \\
\sigma=104\end{array}$ & $\begin{array}{c}99 \\
\sigma=89\end{array}$ & $\begin{array}{c}104 \\
\sigma=99\end{array}$ & $\begin{array}{c}87 \\
\sigma=85\end{array}$ & $\begin{array}{c}89 \\
\sigma=90\end{array}$ & $\begin{array}{c}97 \\
\sigma=73\end{array}$ & $\begin{array}{c}71 \\
\sigma=70\end{array}$ \\
\hline Office/professional & $\begin{array}{c}93 \\
\sigma=68\end{array}$ & $\begin{array}{c}42 \\
\sigma=66\end{array}$ & $\sigma \stackrel{82}{=} 62$ & $\begin{array}{c}72 \\
\sigma=40\end{array}$ & $\begin{array}{c}88 \\
\sigma=52\end{array}$ & $\begin{array}{c}70 \\
\sigma=38\end{array}$ & $\begin{array}{c}58 \\
\sigma=19\end{array}$ & $\sigma=97$ & $\begin{array}{c}143 \\
\sigma=72\end{array}$ & $\begin{array}{c}95 \\
\sigma=43\end{array}$ & $\begin{array}{c}107 \\
\sigma=70\end{array}$ & $\begin{array}{c}66 \\
\sigma=43\end{array}$ & $\begin{array}{c}110 \\
\sigma=93\end{array}$ & $\begin{array}{c}114 \\
\sigma=67\end{array}$ & $\begin{array}{c}68 \\
\sigma=37\end{array}$ \\
\hline Nonrefrig. warehouse & $\begin{array}{c}42 \\
\sigma=56\end{array}$ & $\begin{array}{c}22 \\
\sigma=21\end{array}$ & $\begin{array}{c}16 \\
\sigma=14\end{array}$ & & $\begin{array}{c}22 \\
\sigma=27\end{array}$ & $\sigma \stackrel{21}{=} 19$ & $\begin{array}{c}20 \\
\sigma=37\end{array}$ & $\sigma \stackrel{39}{=} 49$ & $\sigma \stackrel{29}{=26}$ & $\begin{array}{l}37 \\
\sigma=29\end{array}$ & $\begin{array}{c}79 \\
\sigma=83\end{array}$ & $\begin{array}{c}60 \\
\sigma=58\end{array}$ & $\sigma \stackrel{37}{=} 36$ & $\begin{array}{c}58 \\
\sigma= \\
=\end{array}$ & $\begin{array}{c}33 \\
\sigma=31\end{array}$ \\
\hline Education & $\begin{array}{c}83 \\
\sigma=68\end{array}$ & $\sigma=24$ & $\begin{array}{c}73 \\
\sigma=60\end{array}$ & $\begin{array}{c}160 \\
\sigma=73\end{array}$ & $\sigma=62$ & $\begin{array}{c}74 \\
\sigma=70\end{array}$ & $\begin{array}{c}105 \\
\sigma=76\end{array}$ & $\begin{array}{c}102 \\
\sigma=115\end{array}$ & $\begin{array}{c}38 \\
\sigma=22\end{array}$ & $\begin{array}{l}58 \\
\sigma=27\end{array}$ & $\sigma=87$ & $\sigma=45$ & $\begin{array}{c}90 \\
\sigma=52\end{array}$ & $\begin{array}{c}90 \\
\sigma=52\end{array}$ & $\sigma \stackrel{84}{=28}$ \\
\hline Retail (except malls) & $\begin{array}{c}74 \\
\sigma=75\end{array}$ & $\sigma=\frac{61}{=} 52$ & $\begin{array}{c}93 \\
\sigma=135\end{array}$ & $\begin{array}{c}129 \\
\sigma=102\end{array}$ & $\sigma=45$ & $\sigma=30$ & $\sigma=\frac{31}{=} 16$ & $\begin{array}{l}65 \\
\sigma=58\end{array}$ & $\begin{array}{c}100 \\
\sigma=90\end{array}$ & & $\sigma=88$ & $\sigma=49$ & $\begin{array}{c}93 \\
\sigma=86\end{array}$ & $\begin{array}{l}97 \\
\sigma=78\end{array}$ & $\begin{array}{c}102 \\
\sigma=75\end{array}$ \\
\hline Public assembly & $\sigma \stackrel{94}{=85}$ & $\begin{array}{l}75 \\
\sigma=15\end{array}$ & $\begin{array}{c}60 \\
\sigma=63\end{array}$ & & $\begin{array}{c}112 \\
\sigma=137\end{array}$ & $\sigma \stackrel{48}{=} 48$ & $\begin{array}{l}45 \\
\sigma=26\end{array}$ & $\begin{array}{c}110 \\
\sigma=87\end{array}$ & $\begin{array}{c}44 \\
\sigma=31\end{array}$ & $\begin{array}{c}249 \\
\sigma=179\end{array}$ & $\begin{array}{c}103 \\
\sigma=76\end{array}$ & $\sigma=87$ & $\sigma=88$ & $\begin{array}{c}102 \\
\sigma=52\end{array}$ & $\begin{array}{c}97 \\
\sigma=112\end{array}$ \\
\hline Service & $\begin{array}{c}77 \\
\sigma=97\end{array}$ & $\begin{array}{c}60 \\
\sigma=6\end{array}$ & $\begin{array}{c}53 \\
\sigma=50\end{array}$ & & $\begin{array}{c}49 \\
\sigma=50\end{array}$ & $\begin{array}{c}61 \\
\sigma=166\end{array}$ & $\sigma \stackrel{27}{=} 16$ & $\begin{array}{c}82 \\
\sigma=107\end{array}$ & $\begin{array}{c}83 \\
\sigma=90\end{array}$ & & $\begin{array}{c}80 \\
\sigma=77\end{array}$ & $\begin{array}{c}101 \\
\sigma=108\end{array}$ & $\begin{array}{c}88 \\
\sigma=140\end{array}$ & $\begin{array}{c}99 \\
\sigma=62\end{array}$ & $\sigma \stackrel{65}{=69}$ \\
\hline Religious worship & $\begin{array}{c}44 \\
\sigma=34\end{array}$ & & $\sigma \stackrel{31}{=} 11$ & & $\sigma \stackrel{28}{=} 20$ & $\begin{array}{c}31 \\
\sigma=28\end{array}$ & & $\sigma \stackrel{47}{=} 30$ & $\begin{array}{c}56 \\
\sigma=44\end{array}$ & & $\sigma \stackrel{52}{=} 43$ & $\sigma \stackrel{39}{=} 21$ & $\sigma \stackrel{53}{=} 41$ & $\sigma \stackrel{34}{=} 19$ & \\
\hline Lodging & $\begin{array}{c}94 \\
\sigma=63\end{array}$ & $\sigma=\frac{81}{=} 47$ & $\begin{array}{c}91 \\
\sigma=61\end{array}$ & & $\sigma=98$ & $\sigma=57$ & & $\begin{array}{c}92 \\
\sigma=56\end{array}$ & $\begin{array}{c}264 \\
\sigma=80\end{array}$ & $\begin{array}{c}54 \\
\sigma=5\end{array}$ & $\begin{array}{c}89 \\
\sigma=53\end{array}$ & $\sigma \stackrel{65}{=27}$ & $\begin{array}{c}108 \\
\sigma=51\end{array}$ & $\begin{array}{c}93 \\
\sigma=50\end{array}$ & $\sigma=58$ \\
\hline Food services & $\begin{array}{c}258 \\
\sigma=233\end{array}$ & $\begin{array}{c}393 \\
\sigma=133\end{array}$ & $\begin{array}{c}208 \\
\sigma=190\end{array}$ & & $\begin{array}{c}423 \\
\sigma=278\end{array}$ & $\begin{array}{c}393 \\
\sigma=242\end{array}$ & $\begin{array}{c}82 \\
\sigma=21\end{array}$ & $\begin{array}{c}234 \\
\sigma=242 \\
\end{array}$ & & $\begin{array}{c}260 \\
\sigma=199\end{array}$ & $\begin{array}{c}258 \\
\sigma=220\end{array}$ & $\begin{array}{c}228 \\
\sigma=293\end{array}$ & $\begin{array}{c}203 \\
\sigma=188\end{array}$ & $\begin{array}{c}236 \\
\sigma=353 \\
\end{array}$ & $\begin{array}{c}192 \\
\sigma=129\end{array}$ \\
\hline Inpatient health care & $\begin{array}{c}249 \\
\sigma=126\end{array}$ & $\begin{array}{c}200 \\
\sigma=49\end{array}$ & $\begin{array}{c}246 \\
\sigma=88\end{array}$ & $\begin{array}{c}360 \\
\sigma=295\end{array}$ & $\begin{array}{c}205 \\
\sigma=58\end{array}$ & $\begin{array}{c}257 \\
\sigma=203\end{array}$ & $\begin{array}{c}204 \\
\sigma=78\end{array}$ & $\begin{array}{c}248 \\
\sigma=119\end{array}$ & $\begin{array}{c}163 \\
\sigma=71\end{array}$ & & $\begin{array}{c}294 \\
\sigma=147\end{array}$ & $\begin{array}{c}245 \\
\sigma=83\end{array}$ & $\begin{array}{c}240 \\
\sigma=135\end{array}$ & $\begin{array}{c}235 \\
\sigma=108 \\
\end{array}$ & $\begin{array}{c}256 \\
\sigma=57\end{array}$ \\
\hline Public order and safety & $\begin{array}{c}116 \\
\sigma=57\end{array}$ & & $\sigma \stackrel{91}{=} 23$ & & $\begin{array}{c}160 \\
\sigma=126\end{array}$ & $\sigma=21$ & & $\begin{array}{c}129 \\
\sigma=71\end{array}$ & & & $\begin{array}{c}108 \\
\sigma=41\end{array}$ & $\sigma \stackrel{94}{=} 54$ & $\begin{array}{c}126 \\
\sigma=44\end{array}$ & $\begin{array}{l}148 \\
\sigma=3\end{array}$ & \\
\hline Food sales & $\begin{array}{c}200 \\
\sigma=122\end{array}$ & & $\begin{array}{c}166 \\
\sigma=135\end{array}$ & & $\begin{array}{c}212 \\
\sigma=76\end{array}$ & $\begin{array}{c}183 \\
\sigma=93\end{array}$ & $\begin{array}{c}120 \\
\sigma=25\end{array}$ & $\begin{array}{c}242 \\
\sigma=170\end{array}$ & & & $\begin{array}{c}203 \\
\sigma=99\end{array}$ & $\begin{array}{c}147 \\
\sigma=151\end{array}$ & $\sigma=113$ & & $\begin{array}{c}199 \\
\sigma=115\end{array}$ \\
\hline Outpatient health care & $\begin{array}{c}95 \\
\sigma=81\end{array}$ & $\begin{array}{c}19 \\
\sigma=44\end{array}$ & $\begin{array}{c}77 \\
\sigma=49\end{array}$ & & $\stackrel{55}{=}=48$ & $\begin{array}{c}106 \\
\sigma=89\end{array}$ & & $\begin{array}{c}70 \\
\sigma=62\end{array}$ & $\begin{array}{c}190 \\
\sigma=120\end{array}$ & & $\begin{array}{c}111 \\
\sigma=83\end{array}$ & $\begin{array}{c}120 \\
\sigma=85\end{array}$ & $\begin{array}{c}112 \\
\sigma=110\end{array}$ & $\stackrel{91}{=} 80$ & $\begin{array}{c}166 \\
\sigma=73\end{array}$ \\
\hline Vacant & $\sigma \stackrel{21}{\sigma} 31$ & & $\begin{array}{c}4 \\
\sigma=12\end{array}$ & $\begin{array}{c}47 \\
\sigma=21\end{array}$ & $\sigma \stackrel{4}{=} 9$ & $\sigma \stackrel{6}{=} 7$ & $\sigma \stackrel{0}{=} 1$ & $\begin{array}{c}40 \\
\sigma=41\end{array}$ & $\begin{array}{c}3 \\
\sigma=10\end{array}$ & $\begin{array}{c}60 \\
\sigma=12\end{array}$ & $\sigma \stackrel{21}{\sigma=26}$ & $\begin{array}{c}93 \\
\sigma=70\end{array}$ & $\begin{array}{c}22 \\
\sigma=25\end{array}$ & & $\begin{array}{c}55 \\
\sigma=72\end{array}$ \\
\hline Other & $\begin{array}{c}79 \\
\sigma=73\end{array}$ & & $\sigma=58$ & & $\begin{array}{c}100 \\
\sigma=143\end{array}$ & $\begin{array}{c}175 \\
\sigma=110\end{array}$ & & $\sigma=71$ & $\begin{array}{c}26 \\
\sigma=8\end{array}$ & & $\begin{array}{c}94 \\
\sigma=80\end{array}$ & $\sigma \stackrel{92}{=39}$ & $\begin{array}{c}69 \\
\sigma=62\end{array}$ & $\begin{array}{c}85 \\
\sigma=9\end{array}$ & $\begin{array}{c}57 \\
\sigma=32\end{array}$ \\
\hline Skilled nursing & $\begin{array}{c}125 \\
\sigma=63\end{array}$ & & $\begin{array}{c}71 \\
\sigma=34\end{array}$ & & $\sigma \stackrel{84}{=46}$ & $\sigma \stackrel{85}{=74}$ & & $\begin{array}{c}148 \\
\sigma=71\end{array}$ & & & $\begin{array}{c}148 \\
\sigma=56\end{array}$ & $\begin{array}{c}153 \\
\sigma=81\end{array}$ & $\begin{array}{c}118 \\
\sigma=42\end{array}$ & $\begin{array}{c}134 \\
\sigma=76\end{array}$ & \\
\hline Laboratory & $\begin{array}{c}305 \\
\sigma=170\end{array}$ & & & & $\begin{array}{c}242 \\
\sigma=61\end{array}$ & $\begin{array}{c}170 \\
\sigma=82\end{array}$ & & $\begin{array}{c}600 \\
\sigma=367\end{array}$ & & & $\begin{array}{c}370 \\
\sigma=138\end{array}$ & & $\begin{array}{c}268 \\
\sigma=35\end{array}$ & $\begin{array}{c}115 \\
\sigma=15\end{array}$ & \\
\hline Refrigerated warehouse & $\sigma \stackrel{99}{=} 72$ & & & & & & & $\begin{array}{c}120 \\
\sigma=49\end{array}$ & & & $\sigma \stackrel{68}{=} 35$ & $\sigma \stackrel{51}{=} 67$ & $\sigma \stackrel{62}{=} 60$ & & \\
\hline
\end{tabular}


Table 4-2 Existing Stock Models EUI by Subsectors and Climate Zones:

IP Units (kBtu/ft $\left.{ }^{2} \cdot \mathrm{yr}\right)$

\begin{tabular}{|c|c|c|c|c|c|c|c|c|c|c|c|c|c|c|c|}
\hline \multirow[b]{2}{*}{ Subsector } & \multicolumn{15}{|c|}{ Climate Zone } \\
\hline & All & $1 \mathrm{~A}$ & $2 A$ & 2B & $3 A$ & 3B & $3 C$ & 4A & 4B & 4C & $5 A$ & 5B & $6 A$ & 6B & 7 \\
\hline All & $\begin{array}{c}79 \\
\sigma=74\end{array}$ & $\begin{array}{c}102 \\
\sigma=133\end{array}$ & $\begin{array}{c}72 \\
\sigma=76\end{array}$ & $\begin{array}{c}94 \\
\sigma=73\end{array}$ & $\begin{array}{c}78 \\
\sigma=85\end{array}$ & $\begin{array}{c}64 \\
\sigma=70\end{array}$ & $\begin{array}{c}66 \\
\sigma=58\end{array}$ & $\begin{array}{c}79 \\
\sigma=66\end{array}$ & $\begin{array}{c}59 \\
\sigma=39\end{array}$ & $\begin{array}{c}67 \\
\sigma=61\end{array}$ & $\begin{array}{c}86 \\
\sigma=75\end{array}$ & $\begin{array}{c}75 \\
\sigma=61\end{array}$ & $\begin{array}{c}86 \\
\sigma=82\end{array}$ & $\begin{array}{c}87 \\
\sigma=49\end{array}$ & $\begin{array}{c}92 \\
\sigma=85\end{array}$ \\
\hline Office/professional & $\begin{array}{c}79 \\
\sigma=34\end{array}$ & $\begin{array}{c}78 \\
\sigma=17\end{array}$ & $\begin{array}{c}79 \\
\sigma=34\end{array}$ & $\begin{array}{c}73 \\
\sigma=18\end{array}$ & $\begin{array}{c}68 \\
\sigma=24\end{array}$ & $\begin{array}{c}64 \\
\sigma=26\end{array}$ & $\begin{array}{c}63 \\
\sigma=16\end{array}$ & $\begin{array}{c}81 \\
\sigma=36\end{array}$ & $\begin{array}{c}68 \\
\sigma=30\end{array}$ & $\begin{array}{c}68 \\
\sigma=25\end{array}$ & $\begin{array}{c}86 \\
\sigma=36\end{array}$ & $\begin{array}{c}61 \\
\sigma=17\end{array}$ & $\begin{array}{c}85 \\
\sigma=37\end{array}$ & $\begin{array}{c}82 \\
\sigma=36\end{array}$ & $\begin{array}{c}101 \\
\sigma=40\end{array}$ \\
\hline Nonrefrig. warehouse & $\begin{array}{c}34 \\
\sigma=24\end{array}$ & $\begin{array}{c}35 \\
\sigma=29\end{array}$ & $\begin{array}{c}23 \\
\sigma=19\end{array}$ & & $\begin{array}{c}26 \\
\sigma=22\end{array}$ & $\begin{array}{c}30 \\
\sigma=18\end{array}$ & $\begin{array}{c}31 \\
\sigma=36\end{array}$ & $\begin{array}{c}31 \\
\sigma=22\end{array}$ & $\begin{array}{c}31 \\
\sigma=25\end{array}$ & $\begin{array}{c}37 \\
\sigma=16\end{array}$ & $\begin{array}{c}43 \\
\sigma=24\end{array}$ & $\begin{array}{c}40 \\
\sigma=22\end{array}$ & $\begin{array}{c}40 \\
\sigma=31\end{array}$ & $\begin{array}{c}45 \\
\sigma=40\end{array}$ & $\begin{array}{c}42 \\
\sigma=16\end{array}$ \\
\hline Education & $\begin{array}{c}55 \\
\sigma=31\end{array}$ & $\begin{array}{c}96 \\
\sigma=70\end{array}$ & $\begin{array}{c}60 \\
\sigma=46\end{array}$ & $\begin{array}{c}107 \\
\sigma=49\end{array}$ & $\begin{array}{c}42 \\
\sigma=22\end{array}$ & $\begin{array}{c}46 \\
\sigma=20\end{array}$ & $\begin{array}{c}39 \\
\sigma=13\end{array}$ & $\begin{array}{c}57 \\
\sigma=28\end{array}$ & $\begin{array}{c}36 \\
\sigma=14\end{array}$ & $\begin{array}{c}40 \\
\sigma=13\end{array}$ & $\begin{array}{c}55 \\
\sigma=27\end{array}$ & $\begin{array}{c}57 \\
\sigma=27\end{array}$ & $\begin{array}{c}66 \\
\sigma=30\end{array}$ & $\begin{array}{c}63 \\
\sigma=28\end{array}$ & $\begin{array}{c}74 \\
\sigma=11\end{array}$ \\
\hline Retail (except malls) & $\begin{array}{c}75 \\
\sigma=34\end{array}$ & $\begin{array}{c}58 \\
\sigma=62\end{array}$ & $\begin{array}{c}62 \\
\sigma=27\end{array}$ & $\begin{array}{c}93 \\
\sigma=29\end{array}$ & $\begin{array}{c}65 \\
\sigma=30\end{array}$ & $\begin{array}{c}58 \\
\sigma=17\end{array}$ & $\begin{array}{c}57 \\
\sigma=8\end{array}$ & $\begin{array}{c}74 \\
\sigma=31\end{array}$ & $\begin{array}{c}70 \\
\sigma=17\end{array}$ & & $\begin{array}{c}87 \\
\sigma=36\end{array}$ & $\begin{array}{c}77 \\
\sigma=29\end{array}$ & $\begin{array}{c}89 \\
\sigma=39\end{array}$ & $\begin{array}{c}103 \\
\sigma=47\end{array}$ & $\begin{array}{c}136 \\
\sigma=24\end{array}$ \\
\hline Public assembly & $\begin{array}{c}72 \\
\sigma=44\end{array}$ & $\begin{array}{c}73 \\
\sigma=29\end{array}$ & $\begin{array}{c}57 \\
\sigma=47\end{array}$ & & $\begin{array}{c}75 \\
\sigma=41\end{array}$ & $\begin{array}{c}56 \\
\sigma=44\end{array}$ & $\begin{array}{c}55 \\
\sigma=14\end{array}$ & $\begin{array}{c}67 \\
\sigma=29\end{array}$ & $\begin{array}{c}55 \\
\sigma=41\end{array}$ & $\begin{array}{c}99 \\
\sigma=64\end{array}$ & $\begin{array}{c}78 \\
\sigma=44\end{array}$ & $\begin{array}{c}76 \\
\sigma=36\end{array}$ & $\begin{array}{c}84 \\
\sigma=35\end{array}$ & $\begin{array}{c}72 \\
\sigma=20\end{array}$ & $\begin{array}{c}98 \\
\sigma=115\end{array}$ \\
\hline Service & $\begin{array}{c}70 \\
\sigma=36\end{array}$ & $\begin{array}{c}74 \\
\sigma=10\end{array}$ & $\begin{array}{c}56 \\
\sigma=31\end{array}$ & & $\begin{array}{c}53 \\
\sigma=31\end{array}$ & $\begin{array}{c}44 \\
\sigma=31\end{array}$ & $\begin{array}{c}36 \\
\sigma=9\end{array}$ & $\begin{array}{c}71 \\
\sigma=31\end{array}$ & $\begin{array}{c}44 \\
\sigma=14\end{array}$ & & $\begin{array}{c}79 \\
\sigma=34\end{array}$ & $\begin{array}{c}66 \\
\sigma=33\end{array}$ & $\begin{array}{c}69 \\
\sigma=47\end{array}$ & $\begin{array}{c}91 \\
\sigma=29\end{array}$ & $\begin{array}{c}84 \\
\sigma=44\end{array}$ \\
\hline Religious worship & $\begin{array}{c}53 \\
\sigma=43\end{array}$ & & $\begin{array}{c}33 \\
\sigma=16\end{array}$ & & $\begin{array}{c}27 \\
\sigma=19\end{array}$ & $\begin{array}{c}33 \\
\sigma=26\end{array}$ & & $\begin{array}{c}53 \\
\sigma=45\end{array}$ & $\begin{array}{c}76 \\
\sigma=46\end{array}$ & & $\begin{array}{c}69 \\
\sigma=50\end{array}$ & $\begin{array}{c}43 \\
\sigma=21\end{array}$ & $\begin{array}{c}71 \\
\sigma=43\end{array}$ & $\begin{array}{c}52 \\
\sigma=27\end{array}$ & \\
\hline Lodging & $\begin{array}{c}83 \\
\sigma=42\end{array}$ & $\begin{array}{c}102 \\
\sigma=44\end{array}$ & $\begin{array}{c}88 \\
\sigma=31\end{array}$ & & $\begin{array}{c}66 \\
\sigma=26\end{array}$ & $\begin{array}{c}47 \\
\sigma=13\end{array}$ & & $\begin{array}{c}102 \\
\sigma=53\end{array}$ & $\begin{array}{c}65 \\
\sigma=9\end{array}$ & $\begin{array}{c}80 \\
\sigma=56\end{array}$ & $\begin{array}{c}75 \\
\sigma=26\end{array}$ & $\begin{array}{c}82 \\
\sigma=42\end{array}$ & $\begin{array}{c}90 \\
\sigma=41\end{array}$ & $\begin{array}{c}108 \\
\sigma=37\end{array}$ & $\begin{array}{c}78 \\
\sigma=42\end{array}$ \\
\hline Food services & $\begin{array}{c}347 \\
\sigma=124\end{array}$ & $\begin{array}{c}589 \\
\sigma=334\end{array}$ & $\begin{array}{c}326 \\
\sigma=107\end{array}$ & & $\begin{array}{c}324 \\
\sigma=128\end{array}$ & $\begin{array}{c}388 \\
\sigma=75\end{array}$ & $\begin{array}{c}243 \\
\sigma=6\end{array}$ & $\begin{array}{c}338 \\
\sigma=119\end{array}$ & & $\begin{array}{c}462 \\
\sigma=182\end{array}$ & $\begin{array}{c}354 \\
\sigma=131\end{array}$ & $\begin{array}{c}293 \\
\sigma=108\end{array}$ & $\begin{array}{c}371 \\
\sigma=91\end{array}$ & $\begin{array}{c}218 \\
\sigma=90\end{array}$ & $\begin{array}{c}489 \\
\sigma=195\end{array}$ \\
\hline Inpatient health care & $\begin{array}{c}181 \\
\sigma=100\end{array}$ & $\begin{array}{c}197 \\
\sigma=132\end{array}$ & $\begin{array}{c}184 \\
\sigma=79\end{array}$ & $\begin{array}{c}217 \\
\sigma=120\end{array}$ & $\begin{array}{c}143 \\
\sigma=35\end{array}$ & $\begin{array}{c}161 \\
\sigma=48\end{array}$ & $\begin{array}{c}123 \\
\sigma=26\end{array}$ & $\begin{array}{c}175 \\
\sigma=61\end{array}$ & $\begin{array}{c}132 \\
\sigma=13\end{array}$ & & $\begin{array}{c}201 \\
\sigma=93\end{array}$ & $\begin{array}{c}196 \\
\sigma=76\end{array}$ & $\begin{array}{c}234 \\
\sigma=249\end{array}$ & $\begin{array}{c}172 \\
\sigma=57\end{array}$ & $\begin{array}{c}377 \\
\sigma=61\end{array}$ \\
\hline Public order and safety & $\begin{array}{c}80 \\
\sigma=41\end{array}$ & & $\begin{array}{c}61 \\
\sigma=25\end{array}$ & & $\begin{array}{c}59 \\
\sigma=14\end{array}$ & $\begin{array}{c}98 \\
\sigma=31\end{array}$ & & $\begin{array}{c}72 \\
\sigma=37\end{array}$ & & & $\begin{array}{c}96 \\
\sigma=43\end{array}$ & $\begin{array}{c}102 \\
\sigma=74\end{array}$ & $\begin{array}{c}82 \\
\sigma=44\end{array}$ & $\begin{array}{c}101 \\
\sigma=33\end{array}$ & \\
\hline Food sales & $\begin{array}{c}201 \\
\sigma=53\end{array}$ & & $\begin{array}{c}193 \\
\sigma=23\end{array}$ & & $\begin{array}{c}193 \\
\sigma=35\end{array}$ & $\begin{array}{c}154 \\
\sigma=59\end{array}$ & $\begin{array}{c}147 \\
\sigma=32\end{array}$ & $\begin{array}{c}206 \\
\sigma=43\end{array}$ & & & $\begin{array}{c}203 \\
\sigma=56\end{array}$ & $\begin{array}{c}206 \\
\sigma=46\end{array}$ & $\begin{array}{c}259 \\
\sigma=88\end{array}$ & & $\begin{array}{c}214 \\
\sigma=31\end{array}$ \\
\hline Outpatient health care & $\begin{array}{c}88 \\
\sigma=42\end{array}$ & $\begin{array}{c}87 \\
\sigma=22\end{array}$ & $\begin{array}{c}66 \\
\sigma=23\end{array}$ & & $\begin{array}{c}59 \\
\sigma=14\end{array}$ & $\begin{array}{c}92 \\
\sigma=39\end{array}$ & & $\begin{array}{c}77 \\
\sigma=38\end{array}$ & $\begin{array}{c}111 \\
\sigma=58\end{array}$ & & $\begin{array}{c}108 \\
\sigma=53\end{array}$ & $\begin{array}{c}67 \\
\sigma=27\end{array}$ & $\begin{array}{c}97 \\
\sigma=36\end{array}$ & $\begin{array}{c}94 \\
\sigma=34\end{array}$ & $\begin{array}{c}116 \\
\sigma=39\end{array}$ \\
\hline Vacant & $\begin{array}{c}22 \\
\sigma=34\end{array}$ & & $\begin{array}{c}3 \\
\sigma=3\end{array}$ & $\begin{array}{c}36 \\
\sigma=14\end{array}$ & $\begin{array}{c}8 \\
\sigma=9\end{array}$ & $\begin{array}{c}12 \\
\sigma=12\end{array}$ & $\begin{array}{c}2 \\
\sigma=0.04\end{array}$ & $\begin{array}{c}18 \\
\sigma=20\end{array}$ & $\begin{array}{c}3 \\
\sigma=5\end{array}$ & $\begin{array}{c}11 \\
\sigma=23\end{array}$ & $\begin{array}{c}29 \\
\sigma=31\end{array}$ & $\begin{array}{c}17 \\
\sigma=12\end{array}$ & $\begin{array}{c}26 \\
\sigma=38\end{array}$ & & $\begin{array}{c}104 \\
\sigma=95 \\
\end{array}$ \\
\hline Other & $\begin{array}{c}62 \\
\sigma=26 \\
\end{array}$ & & $\begin{array}{c}56 \\
\sigma=35 \\
\end{array}$ & & $\begin{array}{c}39 \\
\sigma=21\end{array}$ & $\begin{array}{c}56 \\
\sigma=17 \\
\end{array}$ & & $\begin{array}{c}67 \\
\sigma=21\end{array}$ & $\begin{array}{c}42 \\
\sigma=0.4\end{array}$ & & $\begin{array}{c}64 \\
\sigma=31 \\
\end{array}$ & $\begin{array}{c}40 \\
\sigma=15\end{array}$ & $\begin{array}{c}61 \\
\sigma=24 \\
\end{array}$ & $\begin{array}{c}104 \\
\sigma=66\end{array}$ & $\begin{array}{c}57 \\
\sigma=36\end{array}$ \\
\hline Skilled nursing & $\begin{array}{c}121 \\
\sigma=26\end{array}$ & & $\begin{array}{c}115 \\
\sigma=6\end{array}$ & & $\begin{array}{c}122 \\
\sigma=40\end{array}$ & $\begin{array}{c}107 \\
\sigma=11\end{array}$ & & $\begin{array}{c}123 \\
\sigma=21\end{array}$ & & & $\begin{array}{c}121 \\
\sigma=21\end{array}$ & $\begin{array}{c}125 \\
\sigma=31\end{array}$ & $\begin{array}{c}120 \\
\sigma=33\end{array}$ & $\begin{array}{c}180 \\
\sigma=33\end{array}$ & \\
\hline Laboratory & $\begin{array}{c}330 \\
\sigma=65\end{array}$ & & & & $\begin{array}{c}352 \\
\sigma=4\end{array}$ & $\begin{array}{c}315 \\
\sigma=95\end{array}$ & & $\begin{array}{c}268 \\
\sigma=54\end{array}$ & & & $\begin{array}{c}327 \\
\sigma=70\end{array}$ & & $\begin{array}{c}334 \\
\sigma=65\end{array}$ & $\begin{array}{c}265 \\
\sigma=74\end{array}$ & \\
\hline Refrigerated warehouse & $\begin{array}{c}86 \\
\sigma=25\end{array}$ & & & & & & & $\begin{array}{c}80 \\
\sigma=7\end{array}$ & & & $\begin{array}{c}70 \\
\sigma=11\end{array}$ & $\begin{array}{c}85 \\
\sigma=34\end{array}$ & $\begin{array}{c}112 \\
\sigma=39\end{array}$ & & \\
\hline
\end{tabular}


Table 4-3 2003 CBECS EUI by Subsectors and Climate Zones

SI Units $\left(\mathrm{MJ} / \mathrm{m}^{2} \cdot \mathrm{yr}\right)$

\begin{tabular}{|c|c|c|c|c|c|c|c|c|c|c|c|c|c|c|c|}
\hline \multirow[b]{2}{*}{ Subsector } & \multicolumn{15}{|c|}{ Climate Zone } \\
\hline & All & $1 \mathrm{~A}$ & $2 A$ & 2B & $3 A$ & $3 B$ & $3 C$ & $4 A$ & 4B & $4 C$ & $5 A$ & $5 B$ & $6 \mathrm{~A}$ & $6 B$ & 7 \\
\hline All & $\sigma=1106$ & $\begin{array}{c}838 \\
\sigma=1118\end{array}$ & $\begin{array}{c}813 \\
\sigma=1001\end{array}$ & $\begin{array}{l}1,290 \\
\sigma=1041\end{array}$ & $\begin{array}{c}1,011 \\
\sigma=1195\end{array}$ & $\begin{array}{c}791 \\
\sigma=1117\end{array}$ & $\begin{array}{c}708 \\
\sigma=618\end{array}$ & $\begin{array}{l}1,076 \\
\sigma=1191\end{array}$ & $\begin{array}{l}1,226 \\
\sigma=1184\end{array}$ & $\begin{array}{l}1,120 \\
\sigma=1016\end{array}$ & $\begin{array}{l}1,186 \\
\sigma=1125\end{array}$ & $\begin{array}{c}986 \\
\sigma=969\end{array}$ & $\begin{array}{l}1,016 \\
\sigma=1021\end{array}$ & $\begin{array}{l}1,097 \\
\sigma=832\end{array}$ & $\begin{array}{c}806 \\
\sigma=801\end{array}$ \\
\hline Office/professional & $\begin{array}{l}1,055 \\
\sigma=773\end{array}$ & $\begin{array}{c}476 \\
\sigma=751\end{array}$ & $\begin{array}{c}930 \\
\sigma=703\end{array}$ & $\begin{array}{c}814 \\
\sigma=451\end{array}$ & $\begin{array}{c}996 \\
\sigma=586\end{array}$ & $\begin{array}{c}798 \\
\sigma=434\end{array}$ & $\begin{array}{c}655 \\
\sigma=214\end{array}$ & $\begin{array}{l}1,099 \\
\sigma=863\end{array}$ & $\begin{array}{l}1,619 \\
\sigma=818\end{array}$ & $\begin{array}{l}1,080 \\
\sigma=489\end{array}$ & $\begin{array}{l}1,219 \\
\sigma=800\end{array}$ & $\begin{array}{c}754 \\
\sigma=488\end{array}$ & $\begin{array}{c}1,253 \\
\sigma=1061\end{array}$ & $\begin{array}{l}1,298 \\
\sigma=757\end{array}$ & $\begin{array}{c}773 \\
\sigma=425\end{array}$ \\
\hline Nonrefrig. warehouse & $\begin{array}{c}481 \\
\sigma=638\end{array}$ & $\begin{array}{c}244 \\
\sigma=239\end{array}$ & $\begin{array}{c}177 \\
\sigma=164\end{array}$ & & $\begin{array}{c}254 \\
\sigma=309\end{array}$ & $\begin{array}{c}243 \\
\sigma=213\end{array}$ & $\begin{array}{c}223 \\
\sigma=421\end{array}$ & $\underset{\sigma=557}{442}$ & $\begin{array}{c}325 \\
\sigma=300\end{array}$ & $\underset{\sigma=332}{422}$ & $\begin{array}{c}897 \\
\sigma=938\end{array}$ & $\begin{array}{c}685 \\
\sigma=661\end{array}$ & $\begin{array}{c}423 \\
\sigma=413\end{array}$ & $\begin{array}{c}655 \\
\sigma=853\end{array}$ & $\begin{array}{c}379 \\
\sigma=356\end{array}$ \\
\hline Education & $\begin{array}{c}944 \\
\sigma=774\end{array}$ & $\begin{array}{c}585 \\
\sigma=269\end{array}$ & $\begin{array}{c}824 \\
\sigma=684\end{array}$ & $\begin{array}{l}1,822 \\
\sigma=834\end{array}$ & $\begin{array}{c}700 \\
\sigma=466\end{array}$ & $\begin{array}{c}846 \\
\sigma=795\end{array}$ & $\begin{array}{l}1,192 \\
\sigma=862\end{array}$ & $\begin{array}{c}1,161 \\
\sigma=1303\end{array}$ & $\begin{array}{c}433 \\
\sigma=252\end{array}$ & $\begin{array}{c}660 \\
\sigma=311\end{array}$ & $\begin{array}{c}988 \\
\sigma=455\end{array}$ & $\begin{array}{c}900 \\
\sigma=512\end{array}$ & $\begin{array}{l}1,026 \\
\sigma=588\end{array}$ & $\begin{array}{l}1,025 \\
\sigma=592\end{array}$ & $\begin{array}{c}949 \\
\sigma=314\end{array}$ \\
\hline Retail (except malls) & $\begin{array}{c}840 \\
\sigma=858\end{array}$ & $\begin{array}{c}690 \\
\sigma=589\end{array}$ & $\begin{array}{c}1,052 \\
\sigma=1533\end{array}$ & $\begin{array}{c}1,464 \\
\sigma=1159\end{array}$ & $\begin{array}{c}680 \\
\sigma=511\end{array}$ & $\begin{array}{c}570 \\
\sigma=449\end{array}$ & $\begin{array}{c}354 \\
\sigma=176\end{array}$ & $\begin{array}{c}742 \\
\sigma=657\end{array}$ & $\begin{array}{c}1,131 \\
\sigma=1021\end{array}$ & & $\begin{array}{c}996 \\
\sigma=1026\end{array}$ & $\begin{array}{c}911 \\
\sigma=559\end{array}$ & $\begin{array}{l}1,057 \\
\sigma=976\end{array}$ & $\begin{array}{l}1,104 \\
\sigma=891\end{array}$ & $\begin{array}{l}1,160 \\
\sigma=847\end{array}$ \\
\hline Public assembly & $\begin{array}{l}1,068 \\
\sigma=967\end{array}$ & $\begin{array}{c}853 \\
\sigma=170\end{array}$ & $\begin{array}{c}684 \\
\sigma=718\end{array}$ & & $\begin{array}{c}1,269 \\
\sigma=1552\end{array}$ & $\begin{array}{c}545 \\
\sigma=544\end{array}$ & $\begin{array}{c}509 \\
\sigma=294\end{array}$ & $\begin{array}{l}1,245 \\
\sigma=990\end{array}$ & $\begin{array}{c}500 \\
\sigma=354\end{array}$ & $\begin{array}{c}2,825 \\
\sigma=2029\end{array}$ & $\begin{array}{l}1,172 \\
\sigma=867\end{array}$ & $\begin{array}{l}1,107 \\
\sigma=992\end{array}$ & $\begin{array}{l}1,004 \\
\sigma=649\end{array}$ & $\begin{array}{l}1,155 \\
\sigma=592\end{array}$ & $\begin{array}{c}1,104 \\
\sigma=1275\end{array}$ \\
\hline Service & $\begin{array}{c}875 \\
\sigma=1108\end{array}$ & $\begin{array}{c}679 \\
\sigma=66\end{array}$ & $\begin{array}{c}604 \\
\sigma=567\end{array}$ & & $\begin{array}{c}562 \\
\sigma=565\end{array}$ & $\begin{array}{c}688 \\
\sigma=1886\end{array}$ & $\begin{array}{c}310 \\
\sigma=180\end{array}$ & $\begin{array}{c}936 \\
\sigma=1217\end{array}$ & $\begin{array}{c}937 \\
\sigma=1017\end{array}$ & & $\begin{array}{c}913 \\
\sigma=874\end{array}$ & $\begin{array}{l}1,150 \\
\sigma=1230\end{array}$ & $\begin{array}{c}995 \\
\sigma=1588\end{array}$ & $\begin{array}{l}1,119 \\
\sigma=705\end{array}$ & $\begin{array}{c}741 \\
\sigma=783\end{array}$ \\
\hline Religious worship & $\begin{array}{c}494 \\
\sigma=391\end{array}$ & & $\begin{array}{c}354 \\
\sigma=126\end{array}$ & & $\begin{array}{c}320 \\
\sigma=232\end{array}$ & $\begin{array}{c}347 \\
\sigma=316\end{array}$ & & $\begin{array}{c}535 \\
\sigma=343\end{array}$ & $\begin{array}{c}635 \\
\sigma=505\end{array}$ & & $\begin{array}{c}591 \\
\sigma=489\end{array}$ & $\begin{array}{c}448 \\
\sigma=237\end{array}$ & $\begin{array}{c}606 \\
\sigma=465\end{array}$ & $\begin{array}{c}386 \\
\sigma=216\end{array}$ & \\
\hline Lodging & $\begin{array}{l}1,070 \\
\sigma=717\end{array}$ & $\begin{array}{c}918 \\
\sigma=536\end{array}$ & $\begin{array}{l}1,037 \\
\sigma=699\end{array}$ & & $\begin{array}{l}1,118 \\
\sigma=504\end{array}$ & $\begin{array}{c}646 \\
\sigma=339\end{array}$ & & $\begin{array}{l}1,050 \\
\sigma=635\end{array}$ & $\begin{array}{l}2,995 \\
\sigma=914\end{array}$ & $\begin{array}{c}617 \\
\sigma=57\end{array}$ & $\begin{array}{l}1,010 \\
\sigma=604\end{array}$ & $\begin{array}{c}740 \\
\sigma=308\end{array}$ & $\begin{array}{l}1,227 \\
\sigma=585\end{array}$ & $\begin{array}{l}1,061 \\
\sigma=571\end{array}$ & $\begin{array}{c}768 \\
\sigma=591\end{array}$ \\
\hline Food services & $\begin{array}{c}2,935 \\
\sigma=2642\end{array}$ & $\begin{array}{c}4,461 \\
\sigma=1514\end{array}$ & $\begin{array}{c}2,367 \\
\sigma=2165\end{array}$ & & $\begin{array}{c}4,803 \\
\sigma=3161\end{array}$ & $\begin{array}{c}4,461 \\
\sigma=2755\end{array}$ & $\begin{array}{c}930 \\
\sigma=238\end{array}$ & $\begin{array}{c}2,660 \\
\sigma=2752\end{array}$ & & $\begin{array}{c}2,950 \\
\sigma=2259\end{array}$ & $\begin{array}{c}2,928 \\
\sigma=2500\end{array}$ & $\begin{array}{c}2,596 \\
\sigma=3327\end{array}$ & $\begin{array}{c}2,310 \\
\sigma=2132\end{array}$ & $\begin{array}{c}2,679 \\
\sigma=4007\end{array}$ & $\begin{array}{c}2,176 \\
\sigma=1471\end{array}$ \\
\hline Inpatient health care & $\begin{array}{c}2,831 \\
\sigma=1432\end{array}$ & $\begin{array}{l}2,270 \\
\sigma=562\end{array}$ & $\begin{array}{c}2,798 \\
\sigma=1005\end{array}$ & $\begin{array}{c}4,094 \\
\sigma=3353\end{array}$ & $\begin{array}{l}2,329 \\
\sigma=655\end{array}$ & $\begin{array}{c}2,920 \\
\sigma=2304\end{array}$ & $\begin{array}{l}2,319 \\
\sigma=888\end{array}$ & $\begin{array}{c}2,820 \\
\sigma=1349\end{array}$ & $\begin{array}{c}1,852 \\
\sigma=808\end{array}$ & & $\begin{array}{c}3,341 \\
\sigma=1667\end{array}$ & $\begin{array}{l}2,785 \\
\sigma=945\end{array}$ & $\begin{array}{c}2,730 \\
\sigma=1538\end{array}$ & $\begin{array}{c}2,675 \\
\sigma=1232\end{array}$ & $\begin{array}{l}2,908 \\
\sigma=650\end{array}$ \\
\hline Public order and safety & $\begin{array}{c}1,316 \\
\sigma=647\end{array}$ & & $\begin{array}{c}1,039 \\
\sigma=264\end{array}$ & & $\begin{array}{c}1,820 \\
\sigma=1429\end{array}$ & $\begin{array}{c}894 \\
\sigma=242\end{array}$ & & $\begin{array}{c}1,468 \\
\sigma=805\end{array}$ & & & $\begin{array}{c}1,229 \\
\sigma \stackrel{=}{=} 460\end{array}$ & $\begin{array}{c}1,062 \\
\sigma \stackrel{=}{1} 609\end{array}$ & $\begin{array}{c}1,437 \\
\sigma=500\end{array}$ & $\begin{array}{l}1,681 \\
\sigma=29\end{array}$ & \\
\hline Food sales & $\begin{array}{c}2,269 \\
\sigma=1388\end{array}$ & & $\begin{array}{l}1,888 \\
\sigma=1537\end{array}$ & & $\begin{array}{c}2,413 \\
\sigma \stackrel{1}{=} 859\end{array}$ & $\begin{array}{c}2,080 \\
\sigma=1060\end{array}$ & $\begin{array}{l}1,364 \\
\sigma=286\end{array}$ & $\begin{array}{c}2,753 \\
\sigma=1927\end{array}$ & & & $\begin{array}{c}2,305 \\
\sigma=1129\end{array}$ & $\begin{array}{c}1,675 \\
\sigma=1717\end{array}$ & $\begin{array}{c}2,745 \\
\sigma=1286\end{array}$ & & $\begin{array}{c}2,257 \\
\sigma=1309\end{array}$ \\
\hline Outpatient health care & $\begin{array}{l}1,075 \\
\sigma=919\end{array}$ & $\begin{array}{l}211 \\
\sigma=505\end{array}$ & $\begin{array}{l}880 \\
\sigma=558\end{array}$ & & $\begin{array}{c}625 \\
\sigma=544\end{array}$ & $\begin{array}{l}1,199 \\
\sigma=1017\end{array}$ & & $\begin{array}{l}790 \\
\sigma=701\end{array}$ & $\begin{array}{c}2,153 \\
\sigma=1361\end{array}$ & & $\begin{array}{l}1,262 \\
\sigma=938\end{array}$ & $\begin{array}{l}1,358 \\
\sigma=964\end{array}$ & $\begin{array}{l}1,267 \\
\sigma=1252\end{array}$ & $\begin{array}{l}1,032 \\
\sigma=911\end{array}$ & $\begin{array}{r}1,887 \\
\sigma=833\end{array}$ \\
\hline Vacant & $\begin{array}{c}237 \\
\sigma=357\end{array}$ & & $\begin{array}{c}42 \\
\sigma=135\end{array}$ & $\sigma=539$ & $\stackrel{45}{\sigma=97}$ & $\sigma \stackrel{71}{=} 75$ & $\sigma \stackrel{2}{=} 9$ & $\begin{array}{c}457 \\
\sigma=463\end{array}$ & $\sigma=34$ & $\begin{array}{l}680 \\
\sigma=137\end{array}$ & $\begin{array}{c}235 \\
\sigma=294\end{array}$ & $\begin{array}{l}1,060 \\
\sigma=799\end{array}$ & $\begin{array}{l}250 \\
\sigma=281\end{array}$ & & $\begin{array}{c}621 \\
\sigma=823\end{array}$ \\
\hline Other & $\begin{array}{c}902 \\
\sigma=827\end{array}$ & & $\begin{array}{l}544 \\
\sigma=675\end{array}$ & & $\begin{array}{c}1,131 \\
\sigma=1624\end{array}$ & $\begin{array}{l}1,987 \\
\sigma=1253\end{array}$ & & $\begin{array}{c}809 \\
\sigma=829\end{array}$ & $\begin{array}{c}290 \\
\sigma=96\end{array}$ & & $\begin{array}{l}1,068 \\
\sigma=906\end{array}$ & $\begin{array}{l}1,044 \\
\sigma=440\end{array}$ & $\begin{array}{c}780 \\
\sigma=702\end{array}$ & $\begin{array}{c}960 \\
\sigma=106\end{array}$ & $\begin{array}{c}643 \\
\sigma=369\end{array}$ \\
\hline Skilled nursing & $\begin{array}{l}1,416 \\
\sigma=715\end{array}$ & & $\begin{array}{l}806 \\
\sigma=391\end{array}$ & & $\begin{array}{c}949 \\
\sigma=524\end{array}$ & $\begin{array}{c}967 \\
\sigma=842\end{array}$ & & $\begin{array}{l}1,687 \\
\sigma=808\end{array}$ & & & $\begin{array}{l}1,686 \\
\sigma=632\end{array}$ & $\begin{array}{l}1,735 \\
\sigma=925\end{array}$ & $\begin{array}{l}1,346 \\
\sigma=477\end{array}$ & $\begin{array}{l}1,517 \\
\sigma=861\end{array}$ & \\
\hline Laboratory & $\begin{array}{l}3,471 \\
\sigma=1928\end{array}$ & & & & $\begin{array}{c}2,752 \\
\sigma=696\end{array}$ & $\begin{array}{l}1,928 \\
\sigma=934\end{array}$ & & $\begin{array}{l}6,816 \\
\sigma=4174\end{array}$ & & & $\begin{array}{c}4,202 \\
\sigma=1564\end{array}$ & & $\begin{array}{c}3,047 \\
\sigma=400\end{array}$ & $\begin{array}{l}1,307 \\
\sigma=171\end{array}$ & \\
\hline Refrigerated warehouse & $\begin{array}{l}1,119 \\
\sigma=815\end{array}$ & & & & & & & $\begin{array}{l}1,361 \\
\sigma=561\end{array}$ & & & $\begin{array}{c}776 \\
\sigma=394\end{array}$ & $\sigma \stackrel{575}{=} 759$ & $\begin{array}{l}707 \\
\sigma=686\end{array}$ & & \\
\hline
\end{tabular}


Table 4-4 Existing Stock Models EUI by Subsectors and Climate Zones:

SI Units (MJ/m².yr)

\begin{tabular}{|c|c|c|c|c|c|c|c|c|c|c|c|c|c|c|c|}
\hline \multirow[b]{2}{*}{ Subsector } & \multicolumn{15}{|c|}{ Climate Zone } \\
\hline & All & $1 \mathrm{~A}$ & $2 A$ & 2B & $3 A$ & 3B & $3 C$ & 4A & 4B & 4C & $5 A$ & $5 B$ & $6 \mathrm{~A}$ & $6 B$ & 7 \\
\hline All & $\begin{array}{c}900 \\
\sigma=843\end{array}$ & $\begin{array}{c}1,154 \\
\sigma=1507\end{array}$ & $\begin{array}{c}814 \\
\sigma=867\end{array}$ & $\begin{array}{c}1,063 \\
\sigma=828\end{array}$ & $\begin{array}{c}887 \\
\sigma=962 \\
\end{array}$ & $\begin{array}{c}730 \\
\sigma=792\end{array}$ & $\begin{array}{c}752 \\
\sigma=659\end{array}$ & $\begin{array}{c}898 \\
\sigma=752 \\
\end{array}$ & $\begin{array}{c}667 \\
\sigma=448\end{array}$ & $\begin{array}{c}759 \\
\sigma=696 \\
\end{array}$ & $\begin{array}{c}982 \\
\sigma=856\end{array}$ & $\begin{array}{c}849 \\
\sigma=693 \\
\end{array}$ & $\begin{array}{c}974 \\
\sigma=933\end{array}$ & $\begin{array}{c}989 \\
\sigma=557\end{array}$ & $\begin{array}{c}1,040 \\
\sigma=961 \\
\end{array}$ \\
\hline Office/professional & $\begin{array}{c}892 \\
\sigma=387\end{array}$ & $\begin{array}{c}886 \\
\sigma=192 \\
\end{array}$ & $\begin{array}{c}896 \\
\sigma=384\end{array}$ & $\begin{array}{c}827 \\
\sigma=204\end{array}$ & $\begin{array}{c}767 \\
\sigma=273\end{array}$ & $\begin{array}{c}730 \\
\sigma=298\end{array}$ & $\begin{array}{c}712 \\
\sigma=181\end{array}$ & $\begin{array}{c}925 \\
\sigma=410\end{array}$ & $\begin{array}{c}767 \\
\sigma=346\end{array}$ & $\begin{array}{c}771 \\
\sigma=288\end{array}$ & $\begin{array}{c}974 \\
\sigma=412\end{array}$ & $\begin{array}{c}693 \\
\sigma=196\end{array}$ & $\begin{array}{c}963 \\
\sigma=417\end{array}$ & $\begin{array}{c}934 \\
\sigma=407\end{array}$ & $\begin{array}{c}1,148 \\
\sigma=454\end{array}$ \\
\hline Nonrefrig. warehouse & $\begin{array}{c}387 \\
\sigma=272 \\
\end{array}$ & $\begin{array}{c}402 \\
\sigma=326 \\
\end{array}$ & $\begin{array}{c}258 \\
\sigma=221\end{array}$ & & $\begin{array}{c}297 \\
\sigma=249\end{array}$ & $\begin{array}{c}336 \\
\sigma=201 \\
\end{array}$ & $\begin{array}{c}356 \\
\sigma=414\end{array}$ & $\begin{array}{c}354 \\
\sigma=255\end{array}$ & $\begin{array}{c}346 \\
\sigma=281 \\
\end{array}$ & $\begin{array}{c}420 \\
\sigma=187 \\
\end{array}$ & $\begin{array}{c}490 \\
\sigma=273 \\
\end{array}$ & $\begin{array}{c}455 \\
\sigma=254 \\
\end{array}$ & $\begin{array}{c}457 \\
\sigma=356 \\
\end{array}$ & $\begin{array}{c}513 \\
\sigma=459 \\
\end{array}$ & $\begin{array}{c}477 \\
\sigma=186 \\
\end{array}$ \\
\hline Education & $\begin{array}{c}628 \\
\sigma=352\end{array}$ & $\begin{array}{c}1,095 \\
\sigma=797\end{array}$ & $\begin{array}{c}677 \\
\sigma=522\end{array}$ & $\begin{array}{c}1,213 \\
\sigma=556\end{array}$ & $\begin{array}{c}479 \\
\sigma=245\end{array}$ & $\begin{array}{c}522 \\
\sigma=231\end{array}$ & $\begin{array}{c}446 \\
\sigma=153\end{array}$ & $\begin{array}{c}649 \\
\sigma=316\end{array}$ & $\begin{array}{c}408 \\
\sigma=163\end{array}$ & $\begin{array}{c}456 \\
\sigma=150\end{array}$ & $\begin{array}{c}627 \\
\sigma=309\end{array}$ & $\begin{array}{c}643 \\
\sigma=309\end{array}$ & $\begin{array}{c}751 \\
\sigma=342\end{array}$ & $\begin{array}{c}710 \\
\sigma=314\end{array}$ & $\begin{array}{c}840 \\
\sigma=130\end{array}$ \\
\hline Retail (except malls) & $\begin{array}{c}848 \\
\sigma=388\end{array}$ & $\begin{array}{c}664 \\
\sigma=708 \\
\end{array}$ & $\begin{array}{c}698 \\
\sigma=302 \\
\end{array}$ & $\begin{array}{c}1,060 \\
\sigma=332\end{array}$ & $\begin{array}{c}739 \\
\sigma=340 \\
\end{array}$ & $\begin{array}{c}664 \\
\sigma=190 \\
\end{array}$ & $\begin{array}{c}642 \\
\sigma=96 \\
\end{array}$ & $\begin{array}{c}845 \\
\sigma=349 \\
\end{array}$ & $\begin{array}{c}793 \\
\sigma=192 \\
\end{array}$ & & $\begin{array}{c}987 \\
\sigma=404\end{array}$ & $\begin{array}{c}874 \\
\sigma=326 \\
\end{array}$ & $\begin{array}{c}1,016 \\
\sigma=441\end{array}$ & $\begin{array}{c}1,172 \\
\sigma=532\end{array}$ & $\begin{array}{c}1,544 \\
\sigma=272 \\
\end{array}$ \\
\hline Public assembly & $\begin{array}{c}821 \\
\sigma=497 \\
\end{array}$ & $\begin{array}{c}824 \\
\sigma=328 \\
\end{array}$ & $\begin{array}{c}646 \\
\sigma=537\end{array}$ & & $\begin{array}{c}846 \\
\sigma=465 \\
\end{array}$ & $\begin{array}{c}631 \\
\sigma=502 \\
\end{array}$ & $\begin{array}{c}624 \\
\sigma=157 \\
\end{array}$ & $\begin{array}{c}755 \\
\sigma=328\end{array}$ & $\begin{array}{c}629 \\
\sigma=464 \\
\end{array}$ & $\begin{array}{c}1,119 \\
\sigma=732\end{array}$ & $\begin{array}{c}884 \\
\sigma=495 \\
\end{array}$ & $\begin{array}{c}862 \\
\sigma=413 \\
\end{array}$ & $\begin{array}{c}955 \\
\sigma=403\end{array}$ & $\begin{array}{c}820 \\
\sigma=222 \\
\end{array}$ & $\begin{array}{c}1,116 \\
\sigma=1306 \\
\end{array}$ \\
\hline Service & $\begin{array}{c}791 \\
\sigma=404\end{array}$ & $\begin{array}{c}836 \\
\sigma=115\end{array}$ & $\begin{array}{c}634 \\
\sigma=352 \\
\end{array}$ & & $\begin{array}{c}600 \\
\sigma=347\end{array}$ & $\begin{array}{c}502 \\
\sigma=350\end{array}$ & $\begin{array}{c}405 \\
\sigma=99\end{array}$ & $\begin{array}{c}802 \\
\sigma=348\end{array}$ & $\begin{array}{c}495 \\
\sigma=159\end{array}$ & & $\begin{array}{c}899 \\
\sigma=383\end{array}$ & $\begin{array}{c}752 \\
\sigma=380\end{array}$ & $\begin{array}{c}778 \\
\sigma=534\end{array}$ & $\begin{array}{c}1,036 \\
\sigma=333\end{array}$ & $\begin{array}{c}954 \\
\sigma=505\end{array}$ \\
\hline Religious worship & $\begin{array}{c}603 \\
\sigma=484\end{array}$ & & $\begin{array}{c}379 \\
\sigma=176\end{array}$ & & $\begin{array}{c}306 \\
\sigma=211\end{array}$ & $\begin{array}{c}375 \\
\sigma=291\end{array}$ & & $\begin{array}{c}603 \\
\sigma=513\end{array}$ & $\begin{array}{c}864 \\
\sigma=523\end{array}$ & & $\begin{array}{c}786 \\
\sigma=572\end{array}$ & $\begin{array}{c}486 \\
\sigma=236\end{array}$ & $\begin{array}{c}801 \\
\sigma=492\end{array}$ & $\begin{array}{c}594 \\
\sigma=309\end{array}$ & \\
\hline Lodging & $\begin{array}{c}947 \\
\sigma=472\end{array}$ & $\begin{array}{c}1,154 \\
\sigma=497\end{array}$ & $\begin{array}{c}996 \\
\sigma=352\end{array}$ & & $\begin{array}{c}753 \\
\sigma=301\end{array}$ & $\begin{array}{c}537 \\
\sigma=150\end{array}$ & & $\begin{array}{c}1,158 \\
\sigma=598\end{array}$ & $\begin{array}{c}735 \\
\sigma=99\end{array}$ & $\begin{array}{c}910 \\
\sigma=640\end{array}$ & $\begin{array}{c}846 \\
\sigma=300\end{array}$ & $\begin{array}{c}931 \\
\sigma=480\end{array}$ & $\begin{array}{c}1,019 \\
\sigma=465\end{array}$ & $\begin{array}{c}1,230 \\
\sigma=423\end{array}$ & $\begin{array}{c}886 \\
\sigma=476 \\
\end{array}$ \\
\hline Food services & $\begin{array}{c}3,944 \\
\sigma=1413\end{array}$ & $\begin{array}{c}6,691 \\
\sigma=3792 \\
\end{array}$ & $\begin{array}{c}3,708 \\
\sigma=1219\end{array}$ & & $\begin{array}{c}3,685 \\
\sigma=1449 \\
\end{array}$ & $\begin{array}{c}4,405 \\
\sigma=851\end{array}$ & $\begin{array}{l}2,765 \\
\sigma=66\end{array}$ & $\begin{array}{c}3,844 \\
\sigma=1348\end{array}$ & & $\begin{array}{c}5,253 \\
\sigma=2067\end{array}$ & $\begin{array}{c}4,021 \\
\sigma=1490\end{array}$ & $\begin{array}{c}3,326 \\
\sigma=1222 \\
\end{array}$ & $\begin{array}{c}4,212 \\
\sigma=1038\end{array}$ & $\begin{array}{c}2,475 \\
\sigma=1026 \\
\end{array}$ & $\begin{array}{c}5,551 \\
\sigma=2217 \\
\end{array}$ \\
\hline Inpatient health care & $\begin{array}{c}2,051 \\
\sigma=1136\end{array}$ & $\begin{array}{c}2,243 \\
\sigma=1505\end{array}$ & $\begin{array}{c}2,094 \\
\sigma=901\end{array}$ & $\begin{array}{c}2,464 \\
\sigma=1363\end{array}$ & $\begin{array}{c}1,627 \\
\sigma=396\end{array}$ & $\begin{array}{c}1,824 \\
\sigma=541\end{array}$ & $\begin{array}{c}1,401 \\
\sigma=290\end{array}$ & $\begin{array}{c}1,992 \\
\sigma=693\end{array}$ & $\begin{array}{c}1,495 \\
\sigma=143\end{array}$ & & $\begin{array}{c}2,288 \\
\sigma=1053\end{array}$ & $\begin{array}{c}2,224 \\
\sigma=861\end{array}$ & $\begin{array}{c}2,659 \\
\sigma=2824\end{array}$ & $\begin{array}{c}1,951 \\
\sigma=645\end{array}$ & $\begin{array}{c}4,280 \\
\sigma=694\end{array}$ \\
\hline Public order and safety & $\begin{array}{c}913 \\
\sigma=462\end{array}$ & & $\begin{array}{c}687 \\
\sigma=280\end{array}$ & & $\begin{array}{c}671 \\
\sigma=161\end{array}$ & $\begin{array}{c}1,108 \\
\sigma=350\end{array}$ & & $\begin{array}{c}813 \\
\sigma=421\end{array}$ & & & $\begin{array}{c}1,086 \\
\sigma=492\end{array}$ & $\begin{array}{c}1,161 \\
\sigma=837\end{array}$ & $\begin{array}{c}934 \\
\sigma=504\end{array}$ & $\begin{array}{c}1,142 \\
\sigma=378\end{array}$ & \\
\hline Food sales & $\begin{array}{c}2,278 \\
\sigma=608\end{array}$ & & $\begin{array}{c}2,195 \\
\sigma=261\end{array}$ & & $\begin{array}{c}2,194 \\
\sigma=396\end{array}$ & $\begin{array}{c}1,747 \\
\sigma=669\end{array}$ & $\begin{array}{c}1,674 \\
\sigma=361\end{array}$ & $\begin{array}{c}2,342 \\
\sigma=491\end{array}$ & & & $\begin{array}{c}2,305 \\
\sigma=641\end{array}$ & $\begin{array}{c}2,338 \\
\sigma=524\end{array}$ & $\begin{array}{c}2,941 \\
\sigma=1001\end{array}$ & & $\begin{array}{c}2,434 \\
\sigma=355\end{array}$ \\
\hline Outpatient health care & $\begin{array}{c}997 \\
\sigma=480\end{array}$ & $\begin{array}{c}988 \\
\sigma=246\end{array}$ & $\begin{array}{c}755 \\
\sigma=263\end{array}$ & & $\begin{array}{c}674 \\
\sigma=157\end{array}$ & $\begin{array}{c}1,049 \\
\sigma=449\end{array}$ & & $\begin{array}{c}873 \\
\sigma=433\end{array}$ & $\begin{array}{c}1,261 \\
\sigma=657\end{array}$ & & $\begin{array}{c}1,223 \\
\sigma=599\end{array}$ & $\begin{array}{c}761 \\
\sigma=302\end{array}$ & $\begin{array}{c}1,106 \\
\sigma=411\end{array}$ & $\begin{array}{c}1,070 \\
\sigma=389\end{array}$ & $\begin{array}{c}1,318 \\
\sigma=438 \\
\end{array}$ \\
\hline Vacant & $\begin{array}{c}244 \\
\sigma=384\end{array}$ & & $\begin{array}{c}31 \\
\sigma=35\end{array}$ & $\begin{array}{c}403 \\
\sigma=156\end{array}$ & $\begin{array}{c}91 \\
\sigma=98\end{array}$ & $\begin{array}{c}136 \\
\sigma=135\end{array}$ & $\begin{array}{c}19 \\
\sigma=0.5\end{array}$ & $\begin{array}{c}208 \\
\sigma=229\end{array}$ & $\begin{array}{c}39 \\
\sigma=61\end{array}$ & $\begin{array}{c}122 \\
\sigma=259\end{array}$ & $\begin{array}{c}325 \\
\sigma=355\end{array}$ & $\begin{array}{c}187 \\
\sigma=134\end{array}$ & $\begin{array}{c}292 \\
\sigma=428\end{array}$ & & $\begin{array}{c}1,176 \\
\sigma=1085\end{array}$ \\
\hline Other & $\begin{array}{c}709 \\
\sigma=294\end{array}$ & & $\begin{array}{c}633 \\
\sigma=398\end{array}$ & & $\begin{array}{c}443 \\
\sigma=233\end{array}$ & $\begin{array}{c}641 \\
\sigma=195\end{array}$ & & $\begin{array}{c}762 \\
\sigma=238\end{array}$ & $\begin{array}{c}474 \\
\sigma=5\end{array}$ & & $\begin{array}{c}727 \\
\sigma=354\end{array}$ & $\begin{array}{c}449 \\
\sigma=170\end{array}$ & $\begin{array}{c}697 \\
\sigma=271\end{array}$ & $\begin{array}{c}1,180 \\
\sigma=756\end{array}$ & $\begin{array}{c}649 \\
\sigma=409\end{array}$ \\
\hline Skilled nursing & $\begin{array}{c}1,375 \\
\sigma=292 \\
\end{array}$ & & $\begin{array}{l}1,302 \\
\sigma=68 \\
\end{array}$ & & $\begin{array}{c}1,389 \\
\sigma=452 \\
\end{array}$ & $\begin{array}{c}1,216 \\
\sigma=126\end{array}$ & & $\begin{array}{c}1,392 \\
\sigma=238 \\
\end{array}$ & & & $\begin{array}{c}1,374 \\
\sigma=242 \\
\end{array}$ & $\begin{array}{c}1,425 \\
\sigma=351\end{array}$ & $\begin{array}{c}1,369 \\
\sigma=372 \\
\end{array}$ & $\begin{array}{c}2,041 \\
\sigma=380\end{array}$ & \\
\hline Laboratory & $\begin{array}{c}3,747 \\
\sigma=733\end{array}$ & & & & $\begin{array}{c}3,998 \\
\sigma=51\end{array}$ & $\begin{array}{c}3,579 \\
\sigma=1077\end{array}$ & & $\begin{array}{c}3,046 \\
\sigma=615\end{array}$ & & & $\begin{array}{c}3,710 \\
\sigma=800\end{array}$ & & $\begin{array}{c}3,792 \\
\sigma=740\end{array}$ & $\begin{array}{c}3,016 \\
\sigma=843\end{array}$ & \\
\hline Refrigerated warehouse & $\begin{array}{c}977 \\
\sigma=286\end{array}$ & & & & & & & $\begin{array}{c}910 \\
\sigma=75\end{array}$ & & & $\begin{array}{c}797 \\
\sigma=120\end{array}$ & $\begin{array}{c}966 \\
\sigma=382\end{array}$ & $\begin{array}{c}1,269 \\
\sigma=447\end{array}$ & & \\
\hline
\end{tabular}


Table 4-5 Number of Samples and Models by Subsectors and Climate Zones

\begin{tabular}{|c|c|c|c|c|c|c|c|c|c|c|c|c|c|c|c|}
\hline Subsector & \multicolumn{15}{|c|}{ Climate Zone } \\
\hline All & 4,820 & 40 & 542 & 24 & 464 & 452 & 52 & 982 & 64 & 50 & 1,152 & 262 & 519 & 120 & 97 \\
\hline Nonrefrig. warehouse & 473 & 5 & 77 & 0 & 49 & 50 & 2 & 93 & 6 & 6 & 94 & 21 & 50 & 10 & 10 \\
\hline Education & 649 & 4 & 91 & 5 & 62 & 59 & 8 & 124 & 9 & 8 & 139 & 58 & 61 & 17 & 4 \\
\hline Public assembly & 279 & 3 & 36 & 0 & 25 & 26 & 3 & 47 & 5 & 5 & 62 & 14 & 37 & 6 & 9 \\
\hline Service & 370 & 2 & 20 & 0 & 43 & 21 & 2 & 76 & 5 & 0 & 100 & 20 & 54 & 13 & 13 \\
\hline Religious worship & 311 & 0 & 31 & 0 & 35 & 29 & 0 & 64 & 6 & 0 & 82 & 11 & 45 & 6 & 0 \\
\hline Lodging & 260 & 7 & 31 & 0 & 20 & 22 & 0 & 58 & 7 & 3 & 51 & 15 & 26 & 8 & 10 \\
\hline Food services & 242 & 2 & 34 & 0 & 27 & 22 & 2 & 49 & 0 & 2 & 57 & 12 & 28 & 2 & 4 \\
\hline Inpatient health care & 144 & 3 & 15 & 0 & 10 & 15 & 0 & 28 & 4 & 0 & 35 & 10 & 12 & 7 & 2 \\
\hline Vacant & 134 & 0 & 17 & 2 & 18 & 10 & 2 & 24 & 3 & 2 & 29 & 3 & 20 & 0 & 3 \\
\hline Other & 64 & 0 & 6 & 0 & 3 & 4 & 0 & 10 & 2 & 0 & 21 & 4 & 8 & 2 & 4 \\
\hline Skilled nursing & 73 & 0 & 5 & 0 & 5 & 7 & 0 & 16 & 0 & 0 & 22 & 6 & 8 & 3 & 0 \\
\hline Laboratory & 43 & 0 & 0 & 0 & 2 & 8 & 0 & 5 & 0 & 0 & 19 & 0 & 4 & 2 & 0 \\
\hline Refrigerated warehouse & 20 & 0 & 0 & 0 & 0 & 0 & 0 & 6 & 0 & 0 & 5 & 3 & 3 & 0 & 0 \\
\hline
\end{tabular}


Table 4-6 Energy Percent Difference between Models and Survey by Subsectors and Climate Zones

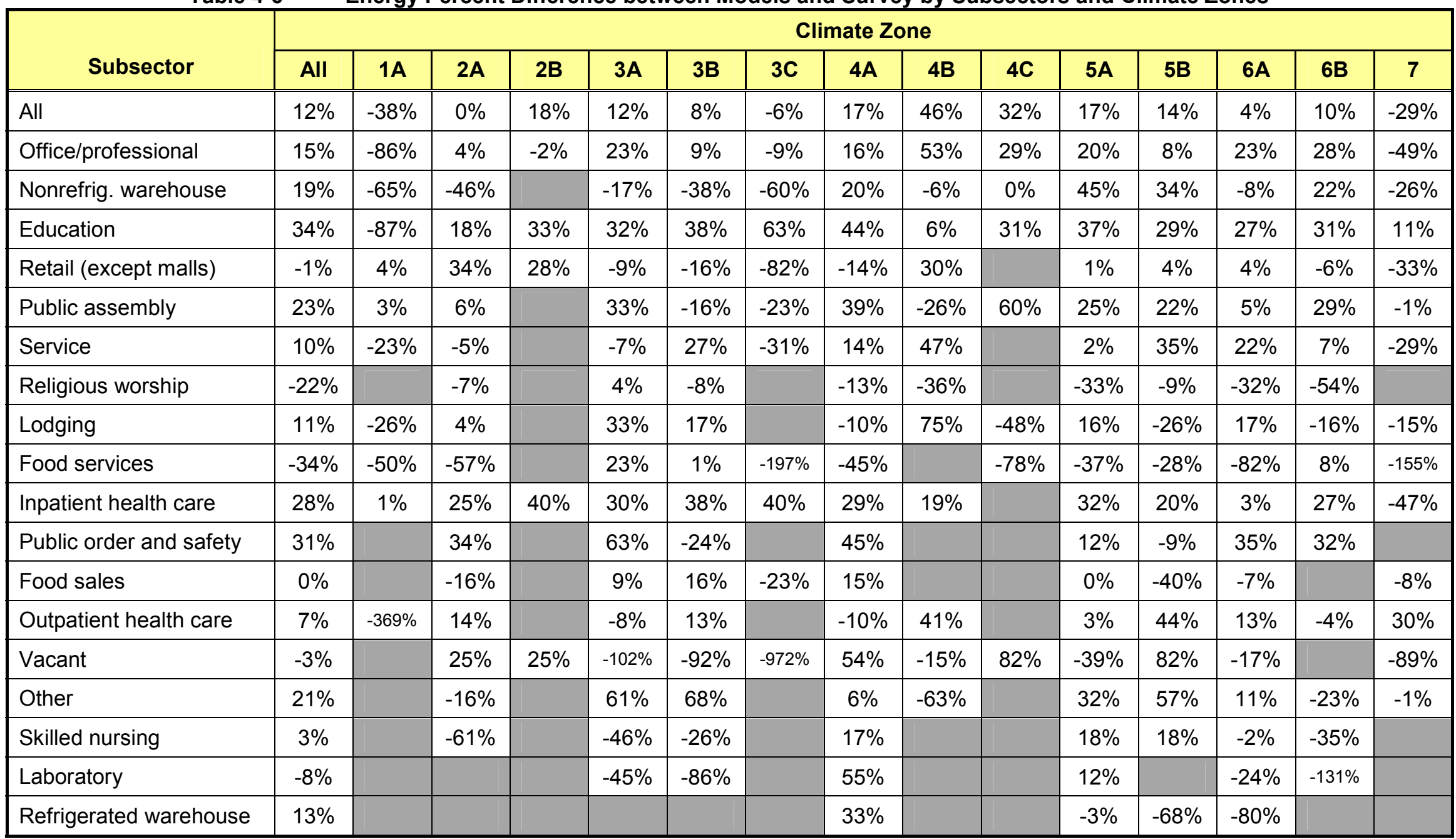


Appendix A also provides plots of the probability density functions (PDFs) for the modeled and survey data by subsector in Figure A-2 to Figure A-20. These PDFs show that EUI varies widely across the sector. The survey results are broader than the models, but the PDFs tend to have the same shapes. Although the simplest way to compare large numbers of results is to look at the averages (weighted mean), the weighted standard deviations and PDFs show that these mean values should be viewed as fairly broad measures. The low number of samples in some climate zones is problematic, because a single model or sample can have an unduly large effect on results for mean values. For example, the PDFs for the 64 buildings in Climate Zone 4B (shown in Figure A-2) reveal that the mean survey results are shifted high by an unusually high fraction at an EUI of around $300 \mathrm{kBtu} / \mathrm{ft}^{2} \cdot \mathrm{yr}$. This was traced to the influence of a single sample building (ID \#5207, a 1 million $\mathrm{ft}^{2}$ hotel with a weighting factor of 134) and demonstrates that a sample size of 64 is probably not sufficient when working with the 2003 CBECS public use data. A similar situation in the Climate Zone 7 classification occurs in the survey where an unusually large weight is put on one sample with a relatively low EUI (ID \#2164, a 1.4 million $\mathrm{ft}^{2}$ warehouse built in 1940 with a weighting factor of 154.5). These examples show the difficulty of generating meaningful weighted mean values with low sample sizes.

Individually, the models and samples do not agree well. Appendix A provides scatter plots that compare total EUI results at the individual building level for each subsector in Figure A-21 to Figure A-39. This scatter is expected because of the lack of data in CBECS and the probabilistic assignments used to generate input. These plots also show a large spread in performance in commercial buildings.

\subsection{Electricity Use}

2003 CBECS survey results include the breakdown by fuel type, and this section focuses on electricity consumed by the whole building. Overall, the models overpredict electricity use by $10 \%$. Figure $4-5$ shows the results for electricity use by subsector. (Table A-7 lists the numeric values.) The models tend to track the survey results fairly well for offices, outpatient health care, food sales, inpatient health care, and public assembly. Subsectors where the model results are far above the survey results include skilled nursing, laboratory, vacant, and religious worship.

Figure 4-6 shows the results for electricity use by climate zone (Table A-8 provides related values). The climate zones that show good agreement include $2 \mathrm{~A}, 3 \mathrm{~A}, 4 \mathrm{~A}$, and $5 \mathrm{~B}$. Relatively poor agreement is observed for Climate Zones 3B, 3C, 6B, and 7A. Interestingly, in Climate Zone 3C the model results for electricity are significantly higher than the survey, but because gas use is underpredicted, the wholebuilding EUI is in much better agreement.

Figure 4-7 shows the results for electricity use by census division (Table A-9 provides values). Census divisions with relatively good agreement include East North Central, South Atlantic, East South Central, and West South Central. Relatively poor agreement is observed for New England, Middle Atlantic, and West North Central. 


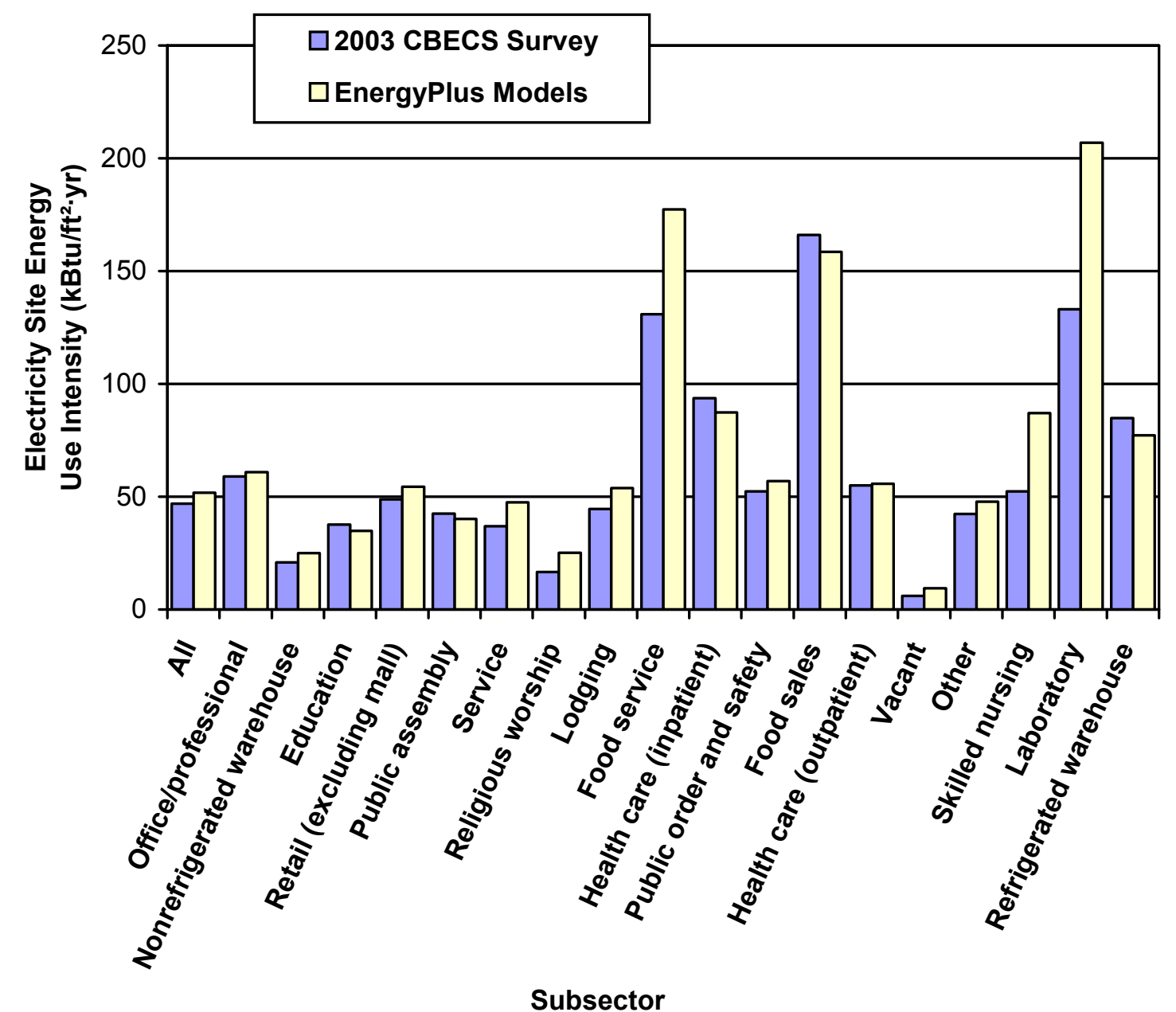

Figure 4-5 Electricity Use Intensity: 2003 CBECS Survey and Modeling by Subsector 


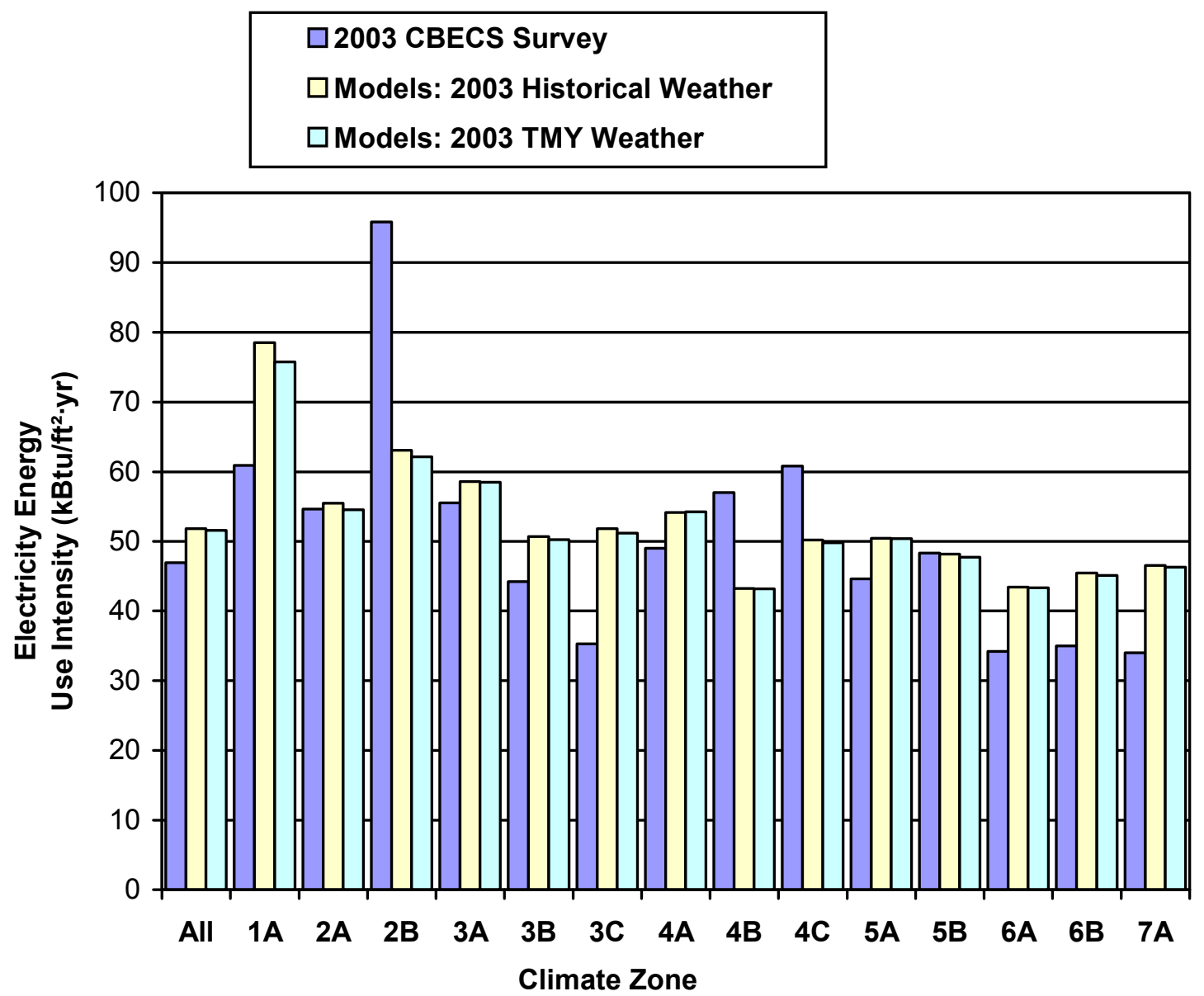

Figure 4-6 Electricity Use Intensity: 2003 CBECS Survey and Modeling by Climate Zone 


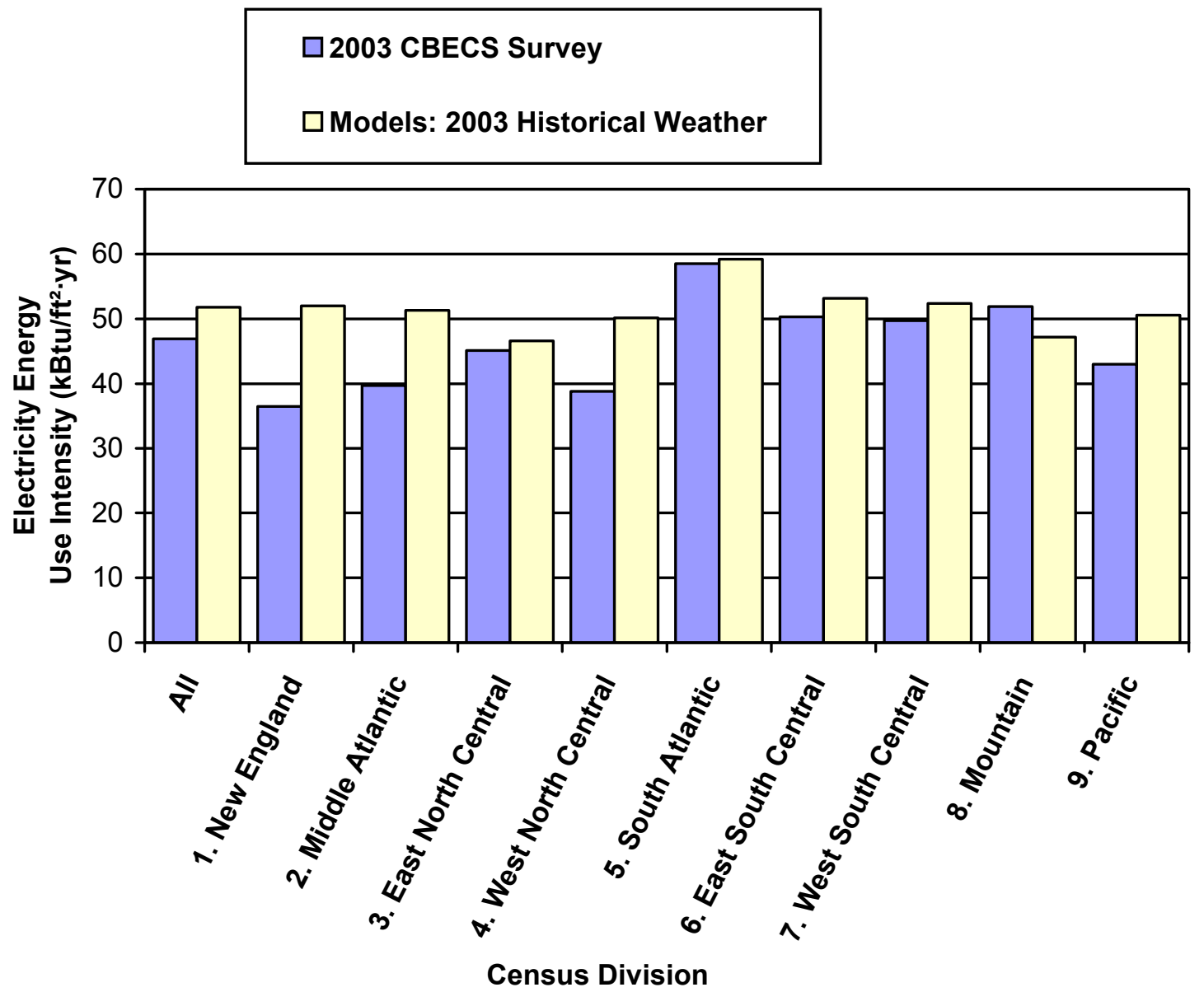

Figure 4-7 Electricity Use Intensity: 2003 CBECS Survey and Modeling by Census Division

\subsection{Natural Gas Use}

2003 CBECS survey results include the breakdown by fuel type; this section focuses on natural gas consumption. Overall, the models underpredict natural gas use by $23 \%$. Figure 4-8 shows the results for natural gas use by subsector (Table A-10 lists the numeric values). Results for natural gas use do not track across subsectors as well as other metrics such as total energy or electricity. Subsectors where the model results are far above the survey results include laboratory, food sales, food services, and religious worship. Subsectors where model results are far below the survey include skilled nursing, other, lodging, education, and nonrefrigerated warehouse.

Figure 4-9 shows the results for natural gas use by climate zone (Table A-11 provides related values). The climate zones that show good agreement include 2A, 2B, 4C, and 6A. Relatively poor agreement is observed for Climate Zones 7A, 4B, and 3B. Interestingly, the survey results by climate zone show some unusual results that are hard to explain, such as relatively high gas use in Climate Zone 4B and relatively low gas use intensity in Climate Zone 7A.

Figure 4-10 shows the results for natural gas use by census division (Table A-12 provides related values). Census divisions with relatively good agreement include South Atlantic. Relatively poor agreement is observed for Mountain, East South Central, West South Central, New England, Middle Atlantic, and East North Central. 


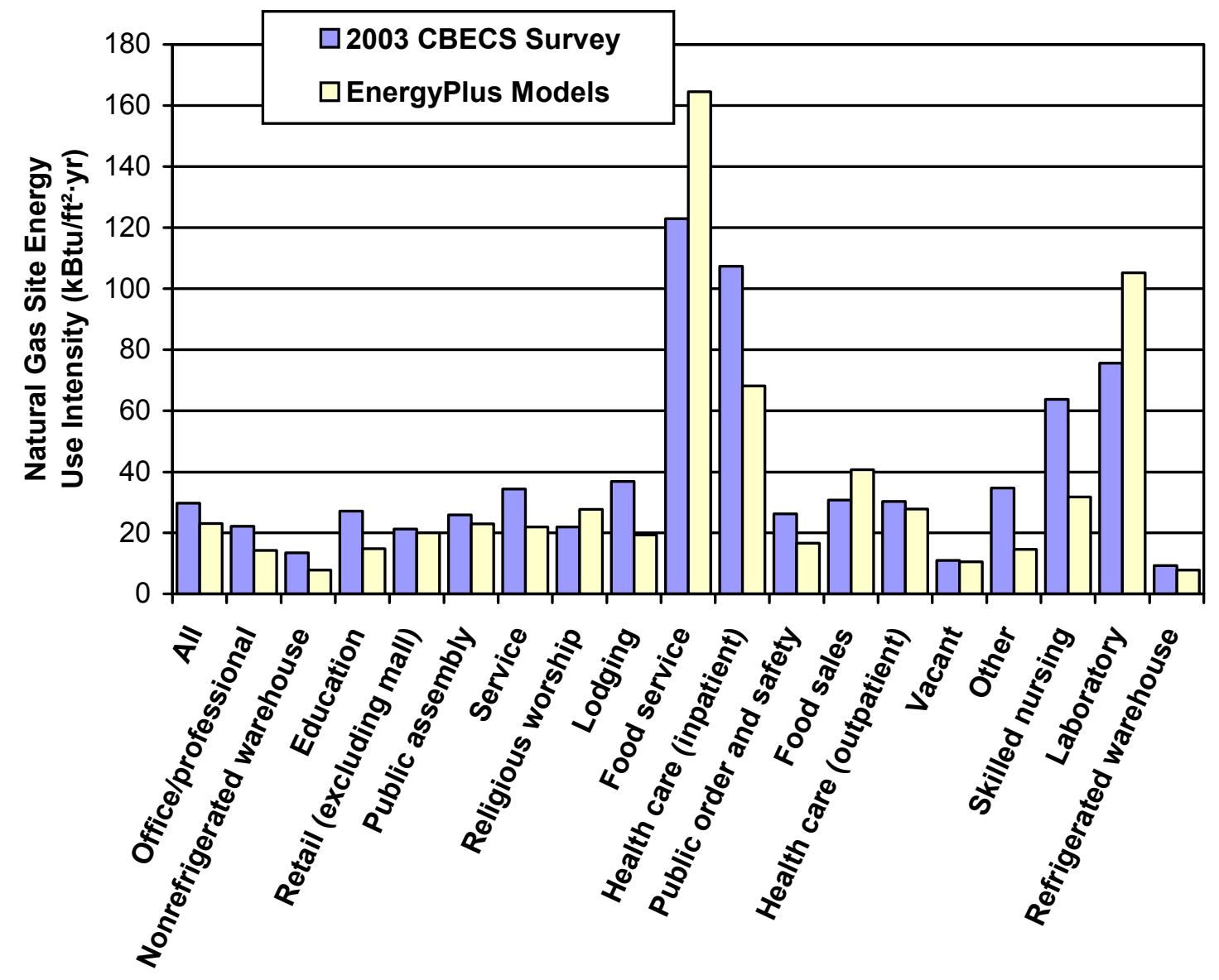

Subsector

Figure 4-8 Natural Gas Use Intensity: 2003 CBECS Survey and Modeling by Subsector 


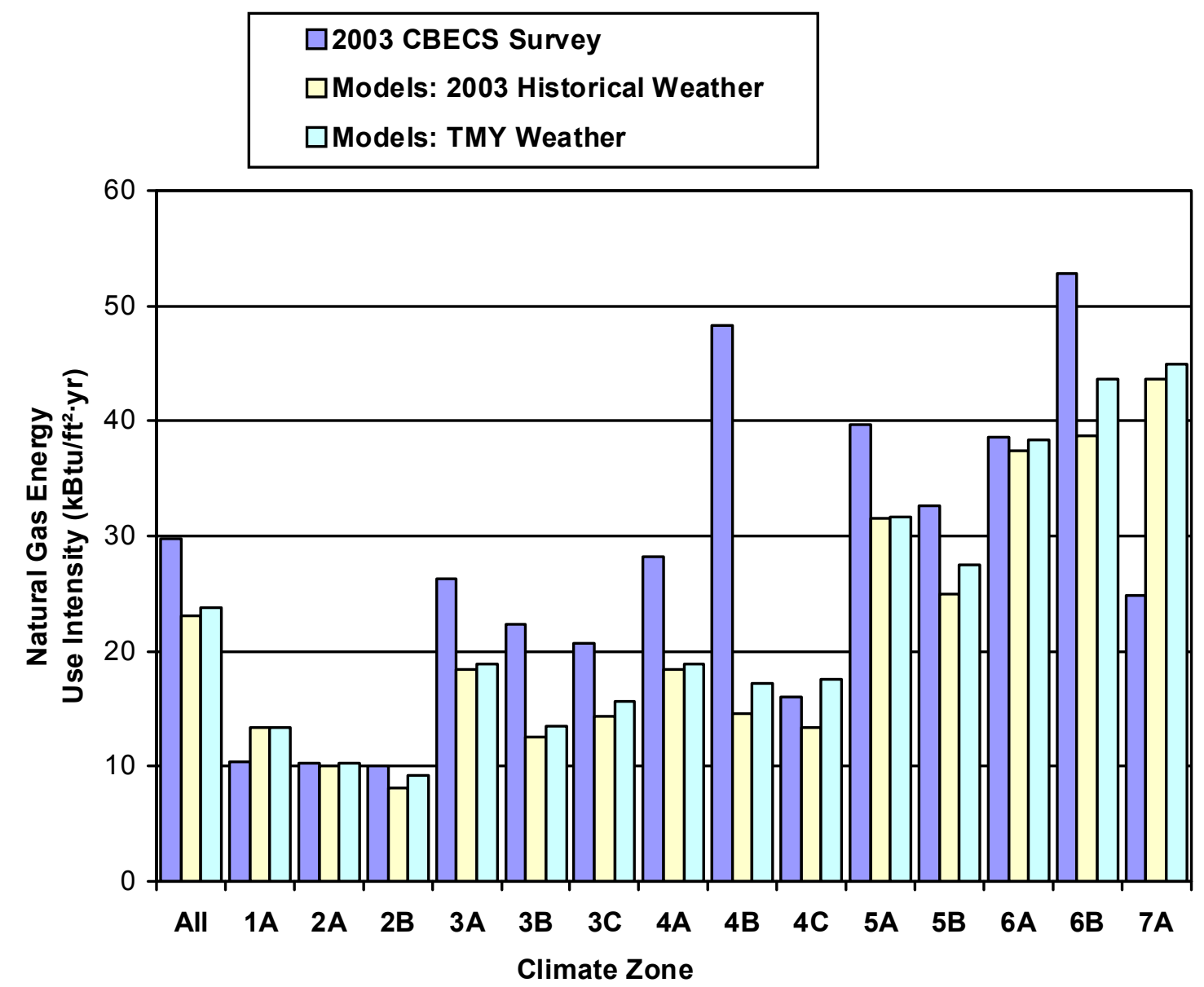

Figure 4-9 Natural Gas Use Intensity: 2003 CBECS Survey and Modeling by Climate Zone 


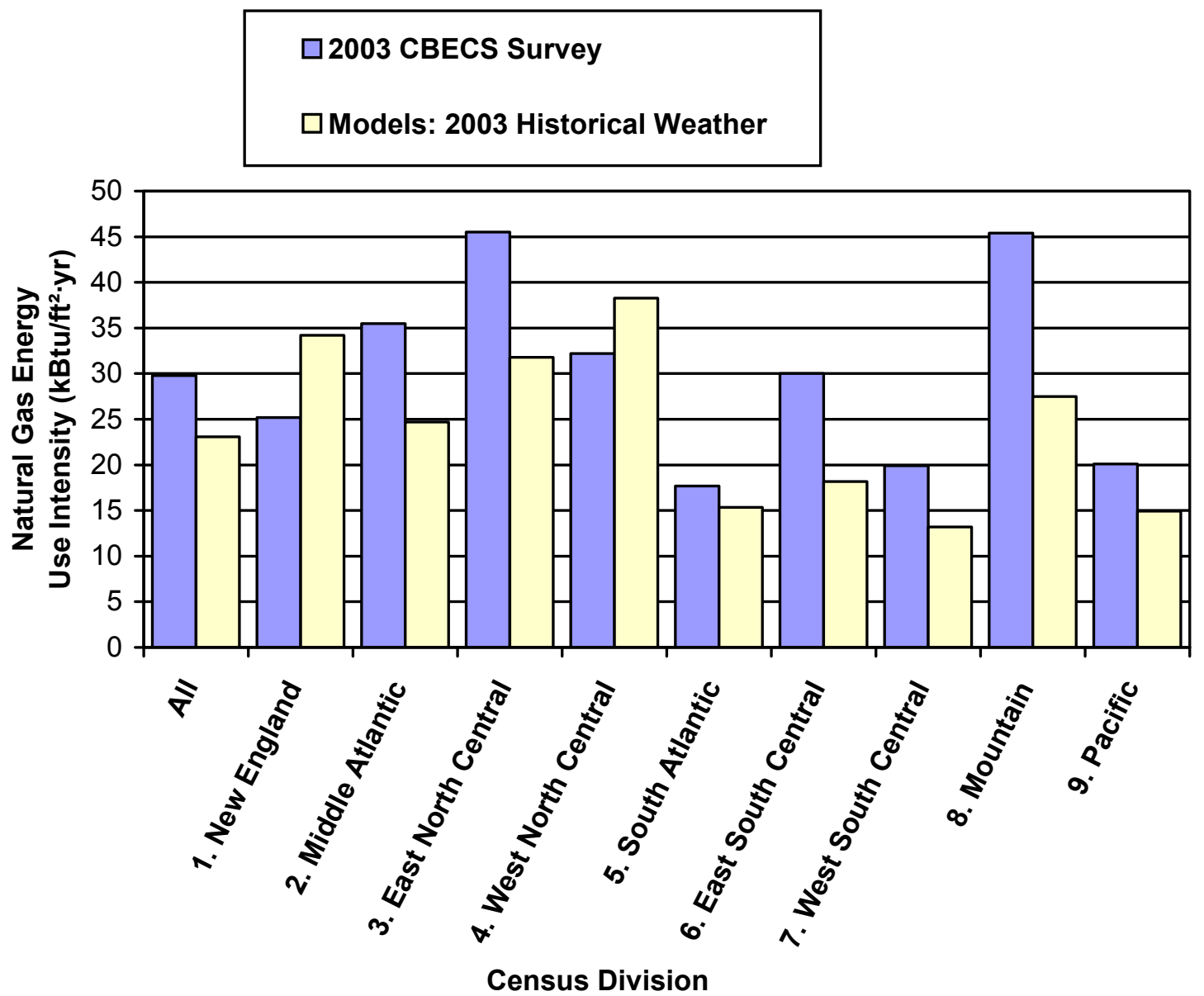

Figure 4-10 Natural Gas Use Intensity: 2003 CBECS Survey and Modeling by Census Division

\subsection{Energy Costs}

2003 CBECS survey results include annual expenditures for energy. Overall, the models overpredict energy costs by $8 \%$. Figure $4-11$ shows the results for energy cost intensity by subsector (Table A-13 lists the numeric values). Results for energy costs track across subsectors fairly well. Relatively good agreement is observed for office, food sales, outpatient health care, inpatient health care, and lodging. Subsectors where the model results are far above the survey results include religious worship, food services, and skilled nursing.

Figure 4-12 shows the results for energy costs by climate zone (Table A-14 provides related values). The climate zones that show good agreement include 1A, 5B, and 6B. Relatively poor agreement is observed for Climate Zones 7A, 4C, and 3C. The survey results by climate zone show some unusual results that are hard to explain, such as relatively high energy cost intensity in Climate Zones 2B and 4C.

Figure 4-13 shows the results for energy costs by census division (Table A-15 provides related values). Census divisions with relatively good agreement include South Atlantic, East South Central, East North Central, and Pacific. Relatively poor agreement is observed for Middle Atlantic, West South Central, and Mountain. 


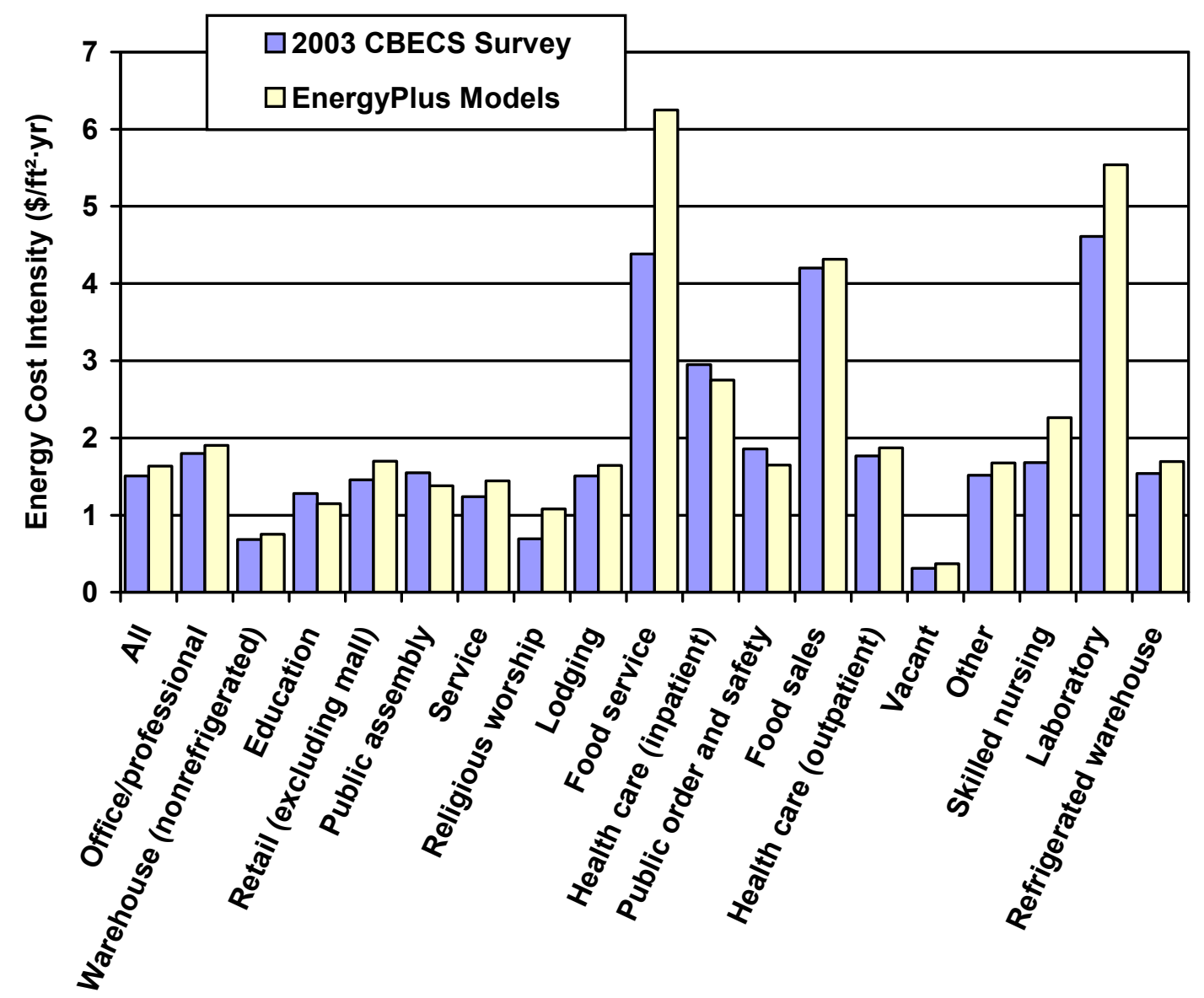

Subsector

Figure 4-11 Energy Cost Intensity: 2003 CBECS Survey and Modeling by Subsector 


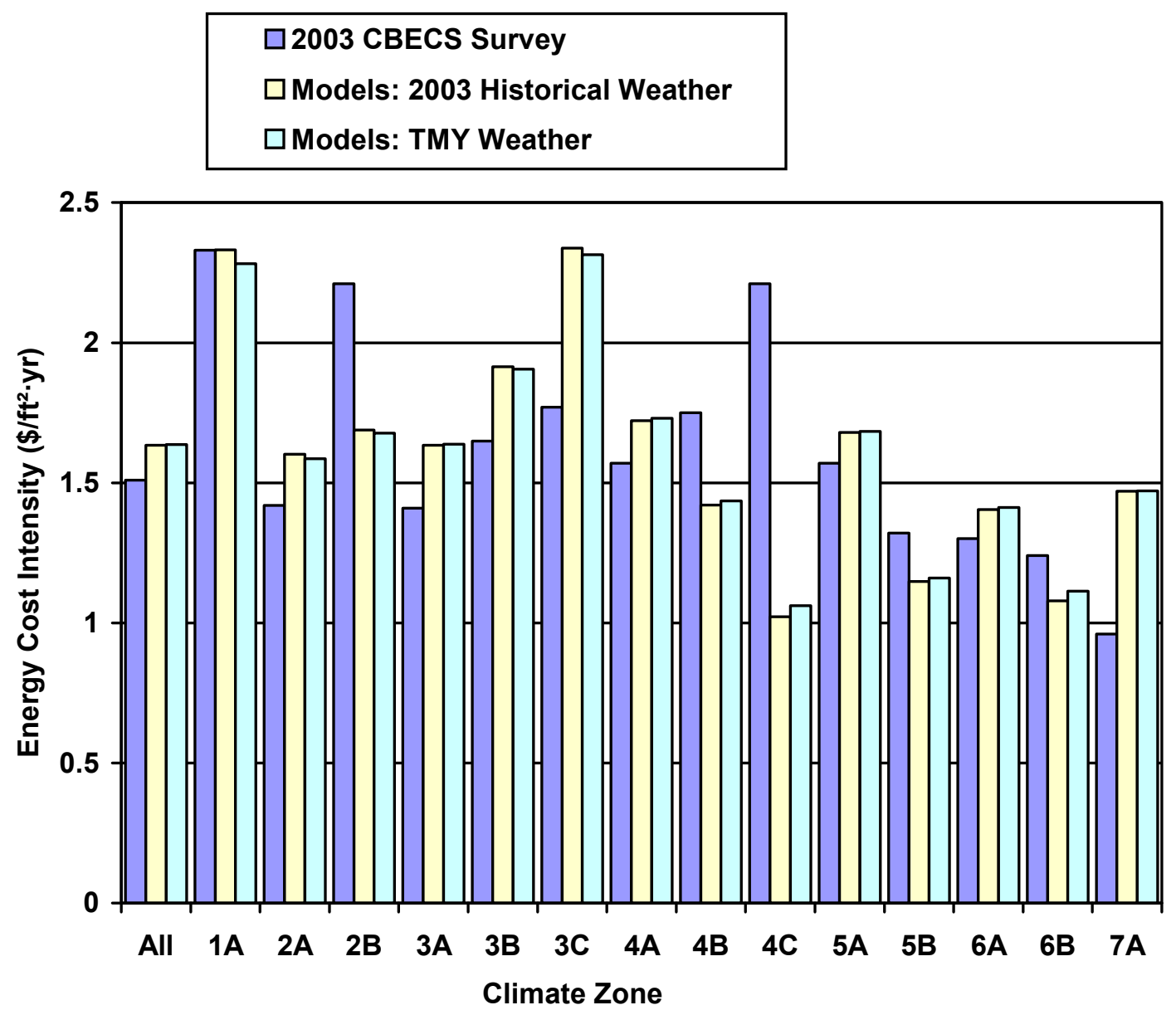

Figure 4-12 Energy Cost Intensity: 2003 CBECS Survey and Modeling by Climate Zone 


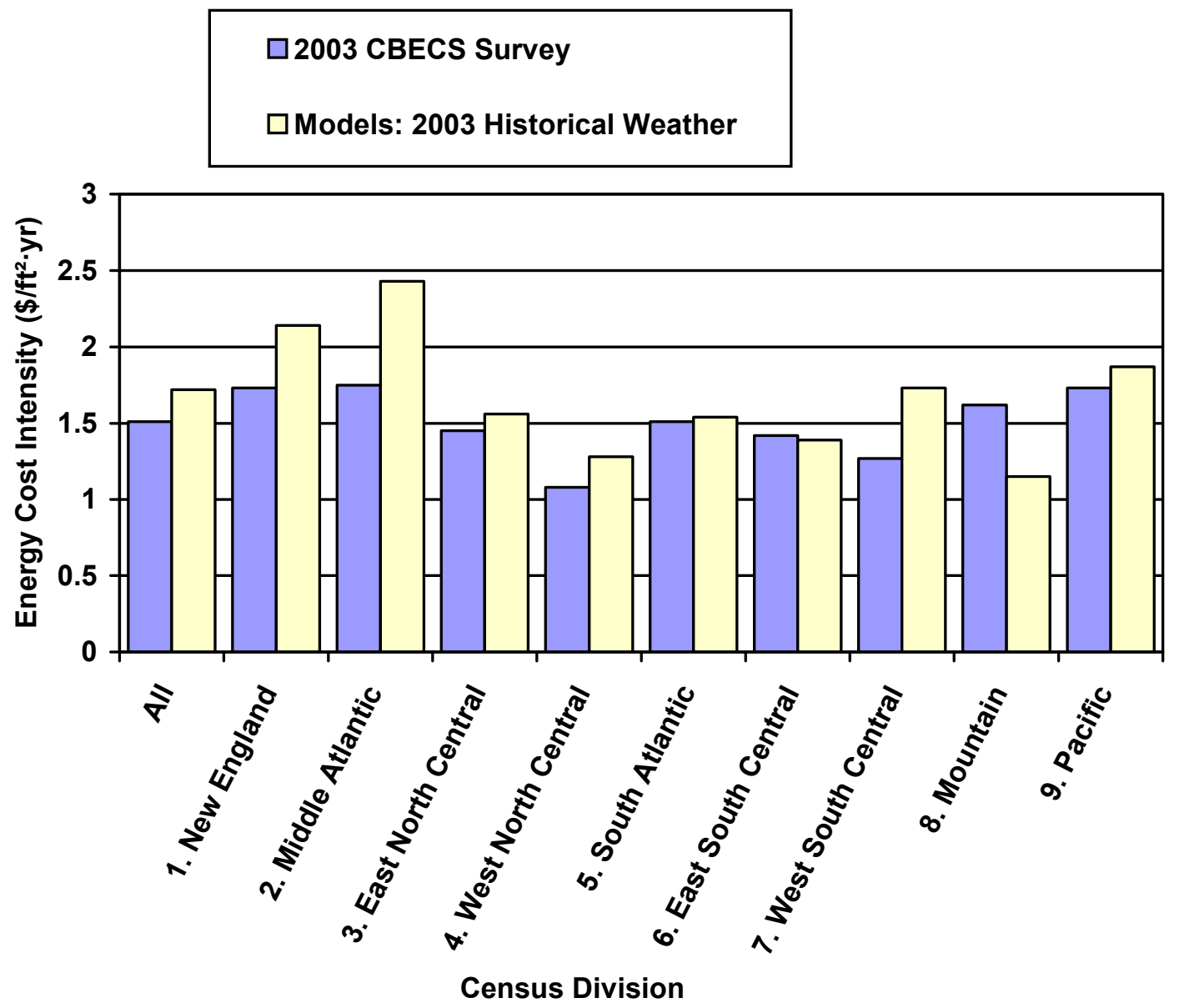

Figure 4-13 Energy Cost Intensity: 2003 CBECS Survey and Modeling by Census Division

\subsection{Literature Comparisons}

Beyond the core source of data from 2003 CBECS, a number of literature sources were used to develop input data for the model. This section presents comparisons between the models and some of the literature data. These comparisons generally involve examining how well the model results actually match the targets for certain loads. Model inputs for loads such as plug and process and outside air were adjusted through an iterative process in an effort to match the data presented in Section 2. But because each iteration is computationally expensive, the process was terminated after eight iterations. This section presents results that examine the levels of agreement.

Table 4-7 lists the electricity end use intensities for plug and process loads. The iterative process for plug and process loads used the methods described in Section C.14 to adjust power density and diversity factors to match the results from CEUS (CEC 2006) listed in Table 2-1. The agreement is fairly good except for the education and refrigerated warehouse subsectors. Plug and process loads for these should be iterated further to improve agreement. Many other subsectors are not represented in CEUS data and so are difficult to judge. 
Table 4-7 Plug and Process Electricity Results from CUES Survey and Modeling

\begin{tabular}{|c|c|c|c|}
\hline Subsector (PBA) & $\begin{array}{c}\text { CEUS Survey } \\
\mathrm{kBtu} / \mathrm{ft}^{2} \cdot \mathrm{yr}\left(\mathrm{MJ} / \mathrm{m}^{2} \cdot \mathrm{yr}\right)\end{array}$ & $\begin{array}{c}\text { Models } \\
\text { kBtu/ft' } \mathrm{ft}^{2} \cdot \mathrm{yr}\left(\mathrm{MJ} / \mathrm{m}^{2} \cdot \mathrm{yr}\right)\end{array}$ & $\begin{array}{l}\text { Percent } \\
\text { Difference }\end{array}$ \\
\hline All & $10.5(119)$ & $10.1(115)$ & $4 \%$ \\
\hline Office/professional & $15.4(175)$ & $14.7(167)$ & $5 \%$ \\
\hline Warehouse & $3.5(40)$ & $3.2(36)$ & $9 \%$ \\
\hline Education & $3.3(38)$ & $4.1(47)$ & $-24 \%$ \\
\hline Retail (except malls) & $6.2(70)$ & $6.4(73)$ & $-3 \%$ \\
\hline Public assembly & $\mathrm{N} / \mathrm{A}$ & $9.3(106)$ & $\mathrm{N} / \mathrm{A}$ \\
\hline Service & $\mathrm{N} / \mathrm{A}$ & $9.6(109)$ & $\mathrm{N} / \mathrm{A}$ \\
\hline Religious worship & $\mathrm{N} / \mathrm{A}$ & $2.7(31)$ & $\mathrm{N} / \mathrm{A}$ \\
\hline Lodging & $8.4(95)$ & $8.6(98)$ & $-2 \%$ \\
\hline Food services & $42.4(482)$ & $33.8(384)$ & $20 \%$ \\
\hline Inpatient health care & $16.4(186)$ & $16.6(189)$ & $-1 \%$ \\
\hline Public order and safety & $\mathrm{N} / \mathrm{A}$ & $22.8(259)$ & $\mathrm{N} / \mathrm{A}$ \\
\hline Food sales & $11.6(132)$ & $10.9(124)$ & $6 \%$ \\
\hline Outpatient health care & $\mathrm{N} / \mathrm{A}$ & $9.4(107)$ & $\mathrm{N} / \mathrm{A}$ \\
\hline Vacant & $\mathrm{N} / \mathrm{A}$ & $3.6(41)$ & $\mathrm{N} / \mathrm{A}$ \\
\hline Other & $10.6(120)$ & $10.5(119)$ & $1 \%$ \\
\hline Skilled nursing & $\mathrm{N} / \mathrm{A}$ & $31.8(361)$ & $\mathrm{N} / \mathrm{A}$ \\
\hline Laboratory & $\mathrm{N} / \mathrm{A}$ & $95.6(1,086)$ & $\mathrm{N} / \mathrm{A}$ \\
\hline Refrigerated warehouse & $9.8(111)$ & $2.2(25)$ & $78 \%$ \\
\hline
\end{tabular}

Table 4-8 lists the electricity end use intensities for commercial refrigeration. The algorithms for generating model inputs for refrigeration use results from CEUS (CEC 2006) listed in Table 2-1 to derive power densities. The agreement is generally fairly good for the individual subsectors (except lodging), but agreement is not as good for all the buildings. This probably stems from CEUS covering a smaller array of the different types of buildings compared to CBECS. 
Table 4-8 Refrigeration Electricity Results from CUES Survey and Modeling

\begin{tabular}{|c|c|c|c|}
\hline Subsector (PBA) & $\begin{array}{c}\text { CEUS Survey Refrigeration } \\
\text { Electricity Intensity } \\
\mathrm{kBtu} / \mathrm{ft}^{2} \cdot \mathrm{yr}\left(\mathrm{MJ} / \mathrm{m}^{2} \cdot \mathrm{yr}\right)\end{array}$ & $\begin{array}{l}\text { Modeled Refrigeration } \\
\text { Electricity Intensity } \\
\mathrm{kBtu} / \mathrm{ft}^{2} \cdot \mathrm{yr}\left(\mathrm{MJ} / \mathrm{m}^{2} \cdot \mathrm{yr}\right)\end{array}$ & $\begin{array}{c}\text { Percent } \\
\text { Difference }\end{array}$ \\
\hline All & $6.2(70)$ & $4.4(50)$ & $29 \%$ \\
\hline Office/professional & $1.6(18)$ & $1.6(18)$ & $0 \%$ \\
\hline Warehouse & $1.0(11)$ & $0.9(10)$ & $10 \%$ \\
\hline Education & $1.7(19)$ & $1.5(17)$ & $12 \%$ \\
\hline Retail (except malls) & $3.5(40)$ & $3.5(40)$ & $0 \%$ \\
\hline Public assembly & $\mathrm{N} / \mathrm{A}$ & $0.7(8)$ & $\mathrm{N} / \mathrm{A}$ \\
\hline Service & $\mathrm{N} / \mathrm{A}$ & $2.7(31)$ & $\mathrm{N} / \mathrm{A}$ \\
\hline Religious worship & $\mathrm{N} / \mathrm{A}$ & $0.7(8)$ & $\mathrm{N} / \mathrm{A}$ \\
\hline Lodging & $3.1(35)$ & $3.8(43)$ & $-23 \%$ \\
\hline Food services & $33.7(383)$ & $33.5(381)$ & $1 \%$ \\
\hline Inpatient health care & $2.4(27)$ & $2.5(28)$ & $-4 \%$ \\
\hline Public order and safety & $\mathrm{N} / \mathrm{A}$ & $1.6(18)$ & $\mathrm{N} / \mathrm{A}$ \\
\hline Food sales & $76.5(869)$ & $75.0(852)$ & $2 \%$ \\
\hline Outpatient health care & $\mathrm{n} / \mathrm{a}$ & $2.2(25)$ & $\mathrm{N} / \mathrm{A}$ \\
\hline Vacant & $\mathrm{N} / \mathrm{A}$ & $0.0(0)$ & $\mathrm{N} / \mathrm{A}$ \\
\hline Other & $2.9(33)$ & $2.6(30)$ & $10 \%$ \\
\hline Skilled nursing & $\mathrm{N} / \mathrm{A}$ & $2.5(28)$ & $\mathrm{N} / \mathrm{A}$ \\
\hline Laboratory & $\mathrm{N} / \mathrm{A}$ & $8.3(94)$ & $\mathrm{N} / \mathrm{A}$ \\
\hline Refrigerated warehouse & $45.9(522)$ & $45.8(520)$ & $0 \%$ \\
\hline
\end{tabular}

Table 4-9 lists the electricity end use intensities for interior lighting. The algorithms for generating model inputs for lighting use data from the U.S. Lighting Market Characterization (DOE 2002) listed in Table 25. The agreement is generally fairly good for the individual subsectors, except for warehouse and vacant. (The comparison for the vacant subsector is not a concern because the literature values seems to be an error.) These differences appear to stem from differences in operating schedules where the current methodology uses a different approach that generates schedules directly from CBECS data. 
Table 4-9 Interior Lighting Electricity Results from U.S. Lighting Market Characterization and Modeling

\begin{tabular}{|c|c|c|c|}
\hline Subsector (PBA) & $\begin{array}{c}\text { U.S. Lighting Market } \\
\text { Characterization } \\
\text { Lighting Electricity Intensity } \\
\mathrm{kBtu} / \mathrm{ft}^{2} \cdot \mathrm{yr}\left(\mathrm{MJ} / \mathrm{m}^{2} \cdot \mathrm{yr}\right)\end{array}$ & $\begin{array}{c}\text { Modeled Lighting } \\
\text { Electricity Intensity } \\
\mathrm{kBtu} / \mathrm{ft}^{2} \cdot \mathrm{yr}\left(\mathrm{MJ} / \mathrm{m}^{2} \cdot \mathrm{yr}\right)\end{array}$ & $\begin{array}{c}\text { Percent } \\
\text { Difference }\end{array}$ \\
\hline All & $\mathrm{N} / \mathrm{A}$ & $18.2(207)$ & $\mathrm{N} / \mathrm{A}$ \\
\hline Office/professional & $22.9(260)$ & $23.2(264)$ & $1 \%$ \\
\hline Warehouse & $16.4(186)$ & $14.4(164)$ & $-12 \%$ \\
\hline Education & $16.7(190)$ & $16.9(192)$ & $1 \%$ \\
\hline Retail (except malls) & $23.9(272)$ & $24.1(274)$ & $1 \%$ \\
\hline Public assembly & $12.3(140)$ & $12.3(140)$ & $0 \%$ \\
\hline Service & $19.4(220)$ & $19.8(225)$ & $2 \%$ \\
\hline Religious worship & $8.5(97)$ & $8.7(99)$ & $2 \%$ \\
\hline Lodging & $16.0(182)$ & $16.2(184)$ & $1 \%$ \\
\hline Food services & $23.9(272)$ & $23.0(261)$ & $-4 \%$ \\
\hline Inpatient health care & $32.4(368)$ & $32.7(372)$ & $1 \%$ \\
\hline Public order and safety & $15.4(175)$ & $15.5(176)$ & $1 \%$ \\
\hline Food sales & $33.4(380)$ & $34.0(386)$ & $2 \%$ \\
\hline Outpatient health care & $19.8(225)$ & $19.7(224)$ & $-1 \%$ \\
\hline Vacant & $25.6(291)$ & $2.1(24)$ & $-92 \%$ \\
\hline Other & $20.8(236)$ & $22.1(251)$ & $6 \%$ \\
\hline Skilled nursing & $18.4(209)$ & $18.5(210)$ & $1 \%$ \\
\hline Laboratory & $28.3(322)$ & $28.6(325)$ & $1 \%$ \\
\hline Refrigerated warehouse & $18.1(206)$ & $18.8(214)$ & $4 \%$ \\
\hline
\end{tabular}

Figure 4-14 plots the distribution of infiltration rates in the models and compares them to the modeling results from Chan (2006). The current study distributions are PDFs that are obtained by using a bin size of $0.05 \mathrm{ACH}$. The PDF for Chan's results was obtained by normalizing the lognormal distribution that corresponds to a geometric mean of 0.35 and a geometric standard deviation of $2.1 \mathrm{ACH}$ (so the area under the curve equals 1.0). The results show reasonable agreement for the most likely value for infiltration at $0.225 \mathrm{ACH}$, but Chan's lognormal distribution has a much longer tail at the higher rates. Chan's distribution is by number of buildings in the sector, whereas the current result's PDF is by floor area in the sector. 


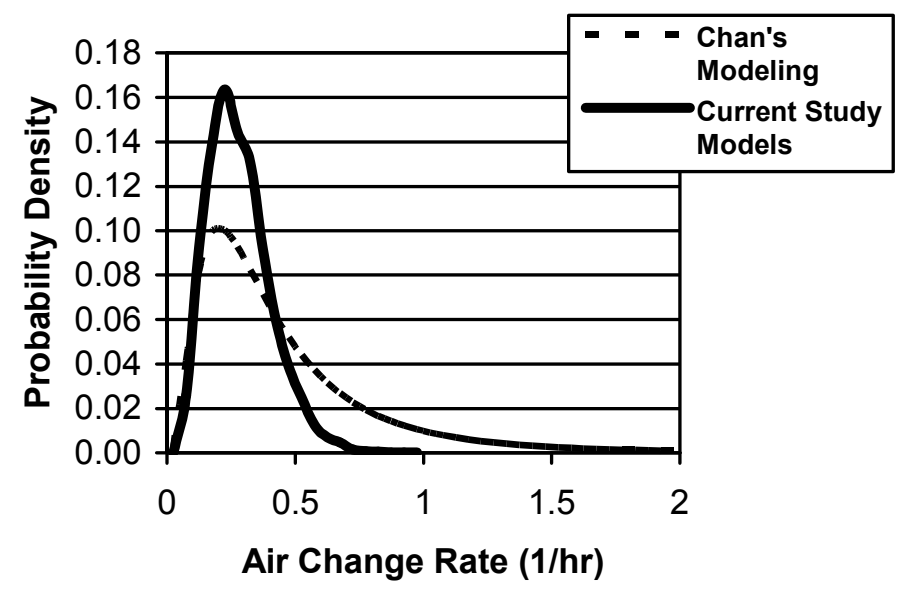

Figure 4-14 PDFs of Infiltration Rates from Chan (2006) and Models

The 2006 Building Energy Data Book (DOE 2006) combines a number of data sources and provides values for end use splits for the year 2004. Because 2003 CBECS does not provide data on end use breakdowns, the best source of national data on end uses appears to be the Building Energy Data Book. Table 4-10 compares literature data and model results for how site energy use is distributed across end use categories. The literature data are mapped to end use categories used in this study and recalculated to remove the SEDS adjustment (SEDS is the State "Energy Consumption, Price, and Expenditure Estimates" and EIA uses this to handle discrepancies between sources of energy data). Here we assume the SEDS adjustment accounts for energy use outside the building envelope such as street lighting. The biggest discrepancy is in energy for service water heating, where the literature data show considerably more energy use. Other differences include the models predicting higher splits for lighting, fans, and space cooling and lower splits for heating and process gas.

Table 4-10 Site Energy End Use Splits from 2006 Building Energy Data Book and Modeling

\begin{tabular}{|l|c|c|c|}
\hline \multicolumn{1}{|c|}{ End Use } & $\begin{array}{c}\text { 2006 Building } \\
\text { Energy Data Book }\end{array}$ & $\begin{array}{c}\text { Existing Stock Models } \\
\text { 2003 Historical } \\
\text { Weather }\end{array}$ & $\begin{array}{c}\text { Existing Stock Models } \\
\text { TMY Weather }\end{array}$ \\
\hline \hline Lighting & $19.9 \%$ & $24.8 \%$ & $24.9 \%$ \\
\hline Space heating & $27.3 \%$ & $22.9 \%$ & $24.0 \%$ \\
\hline Space cooling & $8.8 \%$ & $14.0 \%$ & $13.5 \%$ \\
\hline Refrigeration & $5.2 \%$ & $6.0 \%$ & $6.0 \%$ \\
\hline Water heating & $11.6 \%$ & $3.3 \%$ & $3.4 \%$ \\
\hline Fans & $4.8 \%$ & $8.1 \%$ & $7.2 \%$ \\
\hline Process gas & $9.5 \%$ & $7.2 \%$ & $7.2 \%$ \\
\hline Plug and process electricity & $12.9 \%$ & $13.7 \%$ & $13.8 \%$ \\
\hline
\end{tabular}

\subsection{Historical Weather versus Typical Meteorological Year}

The entire set of 4,820 models was run twice, once with 2003 historical weather data and a second time with TMY weather data. This section compares the results. The modeling uses 181 unique weather locations.

Overall, the results agree to within $0.5 \%$ for total site energy use, $0.4 \%$ for electricity, $3 \%$ for natural gas, and $0.6 \%$ for energy costs. 
The resulting differences between TMY and 2003 historical weather are provided by climate zone for mean total site EUI in Figure 4-2 (and Table A-5), for electricity use intensity in Figure 4-6 (and Table A8), for natural gas use intensity in Figure 4-9 (and Table A-11), and for total energy cost intensity in Figure 4-12 (and Table A-14). The results show relatively minor differences for most climate zones. An example of one of the larger differences is natural gas use for Climate Zone 4C, where 2003 results are $31 \%$ lower than the TMY results.

Figure 4-15 shows an X-Y scatter plot that compares results for all 4,820 buildings with TMY weather on the $\mathrm{X}$-axis and 2003 historical weather on the Y-axis. A simple (unweighted) analysis of the deviations suggests that on average the choice of weather is a $0.9 \%$ effect across the whole sector. However, individual variations go higher and lower, and the average of absolute percent changes indicates that weather is typically a $3.3 \%$ effect on a given building. Some buildings are apparent outliers, but suggest that weather may have a significant effect. Figure 4-16 shows a PDF of the change in EUI between TMY and 2003 historical weather.

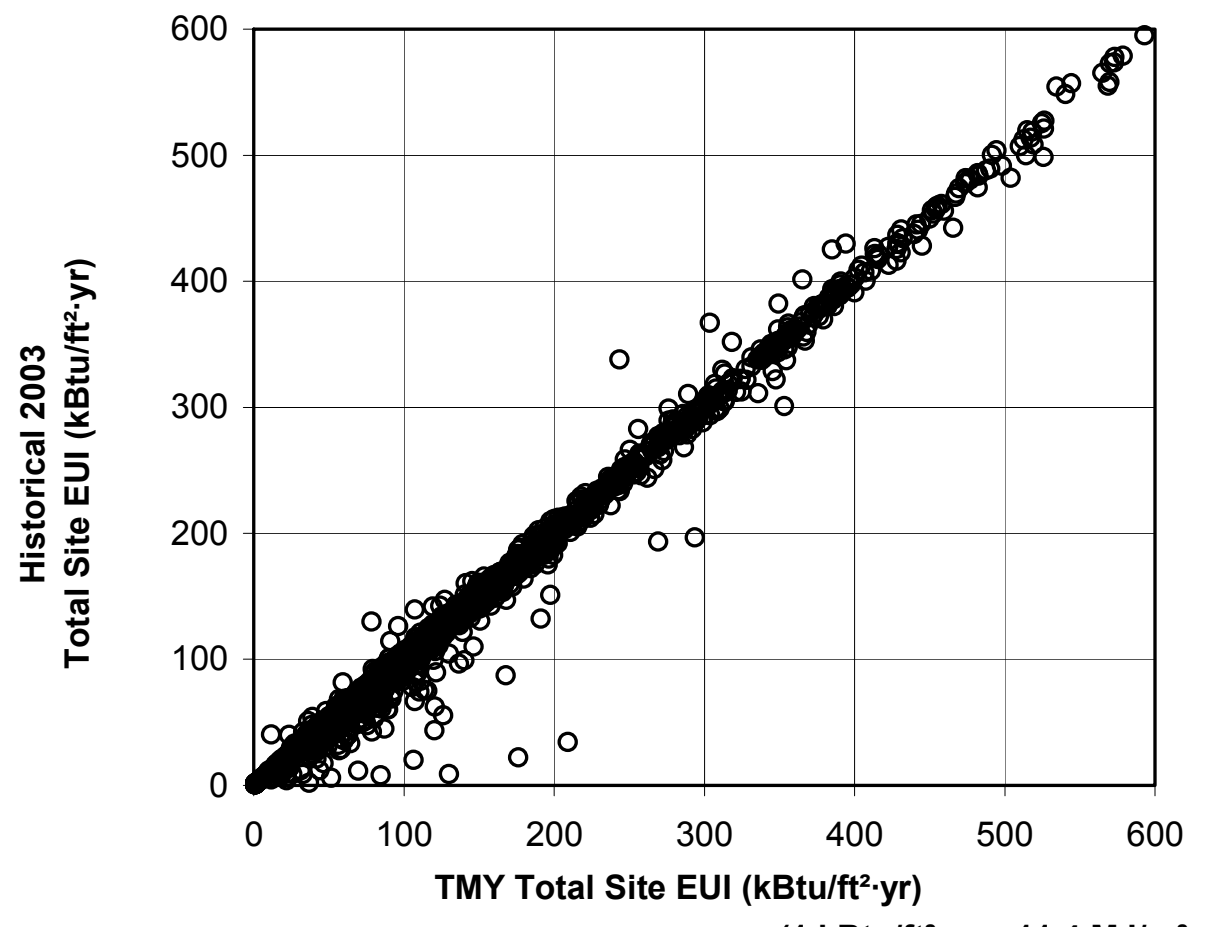

$\left(1 \mathrm{kBtu} / \mathrm{ft}^{2} \cdot \mathrm{yr}=11.4 \mathrm{MJ} / \mathrm{m}^{2} \cdot \mathrm{yr}\right)$

Figure 4-15 Weather Data Scatter Plot: 2003 Historical versus TMY Total EUI 


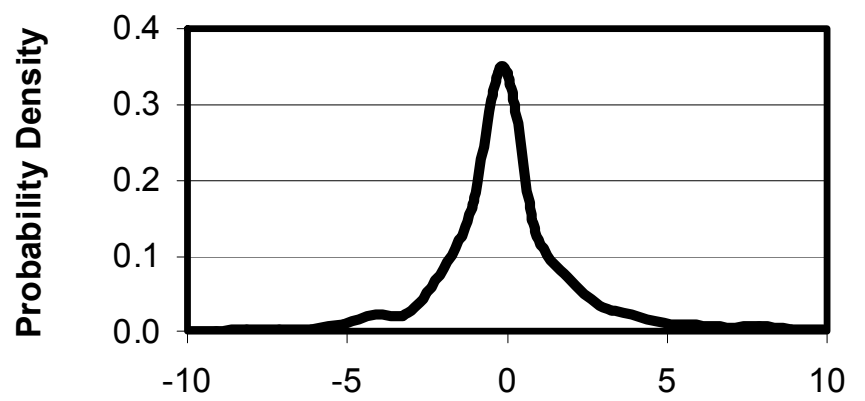

Change in EUI: TMY weather - 2003 weather (kBtu/ft ${ }^{2}-\mathrm{yr}$ )

$\left(1.0 \mathrm{kBtu} / \mathrm{ft}^{2} \cdot \mathrm{yr}=11.4 \mathrm{M} \mathrm{J} / \mathrm{m}^{2} \cdot \mathrm{yr}\right)$

Figure 4-16 PDF of Change in Site EUI from Weather

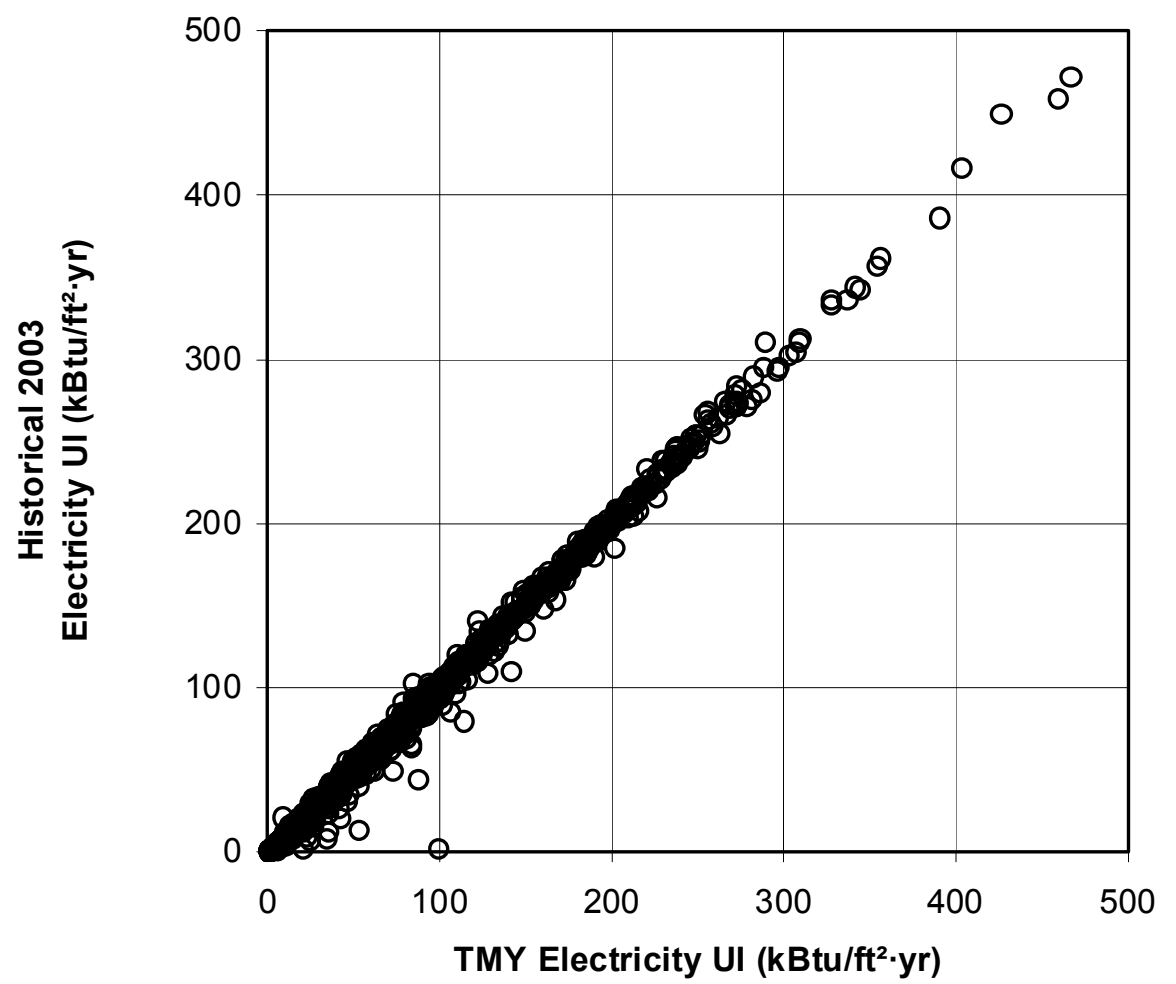

$\left(1 \mathrm{kBtu} / \mathrm{ft}^{2} \cdot \mathrm{yr}=11.4 \mathrm{MJ} / \mathrm{m}^{2} \cdot \mathrm{yr}\right)$

Figure 4-17 Weather Data Scatter Plot: 2003 Historical versus TMY Electricity Use Intensity 


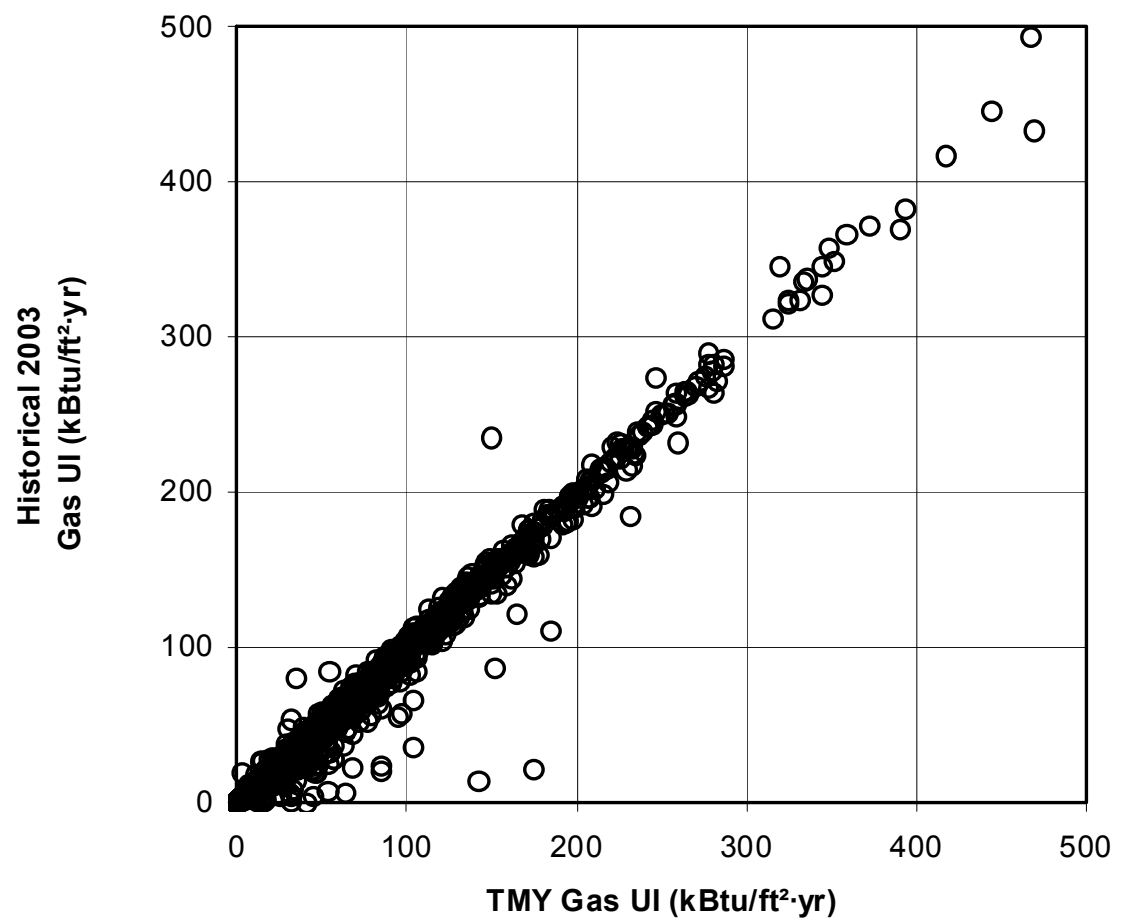

$\left(1 \mathrm{kBtu} / \mathrm{ft}^{2} \cdot \mathrm{yr}=11.4 \mathrm{MJ} / \mathrm{m}^{2} \cdot \mathrm{yr}\right)$

Figure 4-18 Weather Data Scatter Plot: 2003 Historical versus TMY Natural Gas Use Intensity

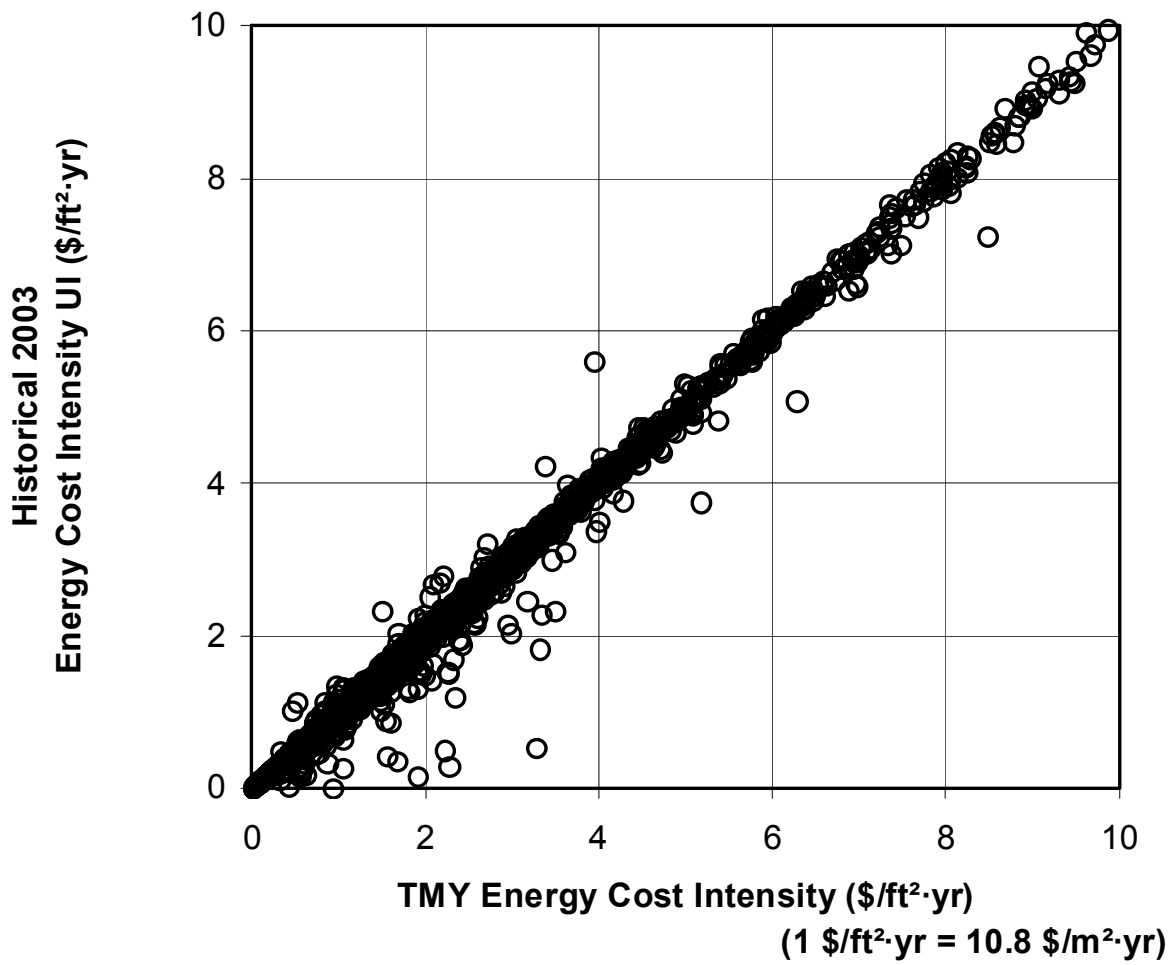

Figure 4-19 Weather Data Scatter Plot: 2003 Historical versus TMY Energy Cost Intensity 


\subsection{Discussion}

This section discusses how well the results from the EnergyPlus models agree with the 2003 CBECS and some of the important lessons learned from conducting the study.

\subsubsection{Validation}

Section 3.3 presented five criteria for determining whether the methodology should be considered valid:

1. The weighted mean results from the models agree with the survey to within one standard deviation of the survey results. This criterion was easily met because the survey data are widely scattered and have relatively large standard deviations. The deviations between model and survey results are well within one standard deviation, and are typically within one-tenth of a weighted standard deviation. Given the wide range of performance levels in the survey data shown by the broad standard deviations, we conclude that the performance levels predicted in the models are generally adequate.

2. The weighted mean results for EUI agree to within $15 \%$. This criterion is met for the sector as a whole; modeling results are $12 \%$ lower than the survey results. Some subsectors do not meet the criteria. The modeling results for education are 34\% too high, food services are $34 \%$ too low, public order and safety are $31 \%$ too high, and inpatient health care is $28 \%$ too low.

3. The mean results for total site electricity use intensity agree to within $20 \%$. This criterion is met for the sector as a whole; modeling results are $10 \%$ over survey. Again, many subsectors do not meet the criteria on their own.

4. The mean results for total site gas use intensity agree to within $20 \%$. This criterion was not met for the sector as a whole; modeling results are 23\% lower than the survey. Natural gas use is underpredicted by more than $40 \%$ in lodging, nonrefrigerated warehouse, education, other, and skilled nursing. The gas criterion was missed by $3 \%$, which in EUI magnitude is only 0.75 $\mathrm{kBtu} / \mathrm{ft}^{2} \cdot \mathrm{yr}\left(8.4 \mathrm{MJ} / \mathrm{m}^{2} \cdot \mathrm{yr}\right)$. Because the weighted standard deviation in the survey results for gas is quite large at $53.8 \mathrm{kBtu} / \mathrm{ft}^{2} \cdot \mathrm{yr}\left(613 \mathrm{MJ} / \mathrm{m}^{2} \cdot \mathrm{yr}\right)$, we conclude that the survey mean results are too imprecise to support such a tight criterion. Because most natural gas consumption is used for space and water heating, and the results shown in Table 4-10 strongly suggest that the models underestimated energy consumption for water heating, the modeling of service water heating should be improved before the method can be applied in subsequent studies that evaluate water heating technologies. Although gas use modeling needs improvement, we conclude that the overall method is still reasonably valid for most technologies.

5. Mean results for energy cost intensity agree to within $15 \%$. This criterion is met for the sector as a whole; the model results are $8 \%$ higher than the survey. Because the results for both energy costs and total energy use align reasonably well with the survey results, we conclude that the utility tariff input data and EnergyPlus calculations are reasonably valid.

\subsubsection{Sector Representation}

The results can also be examined in terms of how well the modeling reflects how energy performance is distributed across the sector. The mean values tell one fairly simple story; but the distributions have more involved information about the widely varying sector. The models have more central tendency than the survey. The weighted standard deviations show that both the survey and models are broadly distributed, but that the models are narrower than the survey. The survey results for EUI show more variability than the models; the survey shows a weighted standard deviation in total EUI of $97.4 \mathrm{kBtu} / \mathrm{ft}^{2} \cdot \mathrm{yr}(1,107$ $\left.\mathrm{MJ} / \mathrm{m}^{2} \cdot \mathrm{yr}\right)$, and the models $79.6 \mathrm{kBtu} / \mathrm{ft}^{2} \cdot \mathrm{yr}\left(904 \mathrm{MJ} / \mathrm{m}^{2} \cdot \mathrm{yr}\right)$. The PDFs in Appendix A also show more central tendency, but overall, the distributions compare fairly well for subsets with large numbers of models and samples. Based on comparing PDFs of the modeling and the survey, we conclude that the 
modeling methodology is a reasonably valid method of representing the entire commercial sector and that the models show less scatter than does the survey.

\subsubsection{Critical Review}

Although results show that the methodology is reasonably valid, the modeling could be improved. Basing the methodology on EnergyPlus means that there is almost no limit to how much the individual models could be improved in terms of detail and accuracy. The models in this study are simple compared to the sort of models that are typical for analyzing individual building projects. This simplicity stems primarily from the limited data available on the sample buildings from CBECS, but also from the practical challenges of creating and working with such large numbers of EnergyPlus input files. Nevertheless, the modeling methods and input assumptions could be improved in numerous ways and the following are, in no particular order, high-priority areas for improvement that have been identified through the course of this research project and critical review.

- The energy consumption results from 2003 CBECS are a rich source of data about the individual samples. The effort to use the energy results to validate data hinders their use in developing improved input on an individual basis. Future efforts should consider abandoning the blind validation approach and use the energy consumption data directly in an iterative process to improve each model.

- The simplification that buildings are either completely conditioned or not conditioned should be relaxed so that the survey responses for percent conditioned are used directly. The input file generation routines could be expanded to model the buildings with nonuniform conditioning systems and internal gains.

- The results for natural gas shown in Section 4.3 indicate that improvements need to be made in the modeling of natural gas process loads. The results suggest that gas-fired process loads are significantly underrepresented in subsectors such as laboratory, skilled nursing, health care, and food service. Although service water heating is included in the models, independent data about the rates of hot water use are scant. Additional literature searching and new research are needed to provide independent guidance about how to improve inputs for service water heating. The service water heating inputs need to be improved before the methodology can be considered appropriate for subsequent study of water heating technologies.

- The schedules created from CBECS data variables lack diversity and realism. The profiles used to create schedules are generally square-shaped, or "top hat," and do not reflect the shapes and magnitudes observed anecdotally in real buildings. Improved methods are needed to generate schedules that are more realistic.

- HVAC component performance levels are based on assumption, and more research is needed to understand the installed performance of components in existing buildings. For example, fan efficiencies are probably too high; the model inputs should be revised to use lower efficiencies for fans. Building faults (duct leakage, improper schedules, low refrigerant charge, economizer issues, etc.) are also a significant issue with serious modeling challenges — see for example TIAX (2005).

- HVAC system topologies are simplified and automatically sized. More detailed designs for systems and discrete equipment sizes would be an improvement.

- The assumption that the weighting factors from CBECS still apply to the synthetic buildings generated by probabilistic assignments could be investigated by oversampling with the models. The inputs are assigned by using density functions on a building-by-building case; the building weighting factors are applied to the outputs. Hence, the aggregated results could be skewed by particularly extreme random assignments that happen to be for buildings with high weighting 
factors. This and other effects of the random assignments for input could be investigated further by repeatedly generating and running entire sets of models and examining how results vary.

However, this would require enormous computational resources.

- The weighted standard deviations of the CBECS data are relatively large, in some cases larger than the mean. The analysis of PDFs shows that EUIs are not distributed normally. Future analysis should consider EUIs as log-normal rather than normal distributions and use weighted geometric mean and weighted geometric standard deviations to characterize data sets.

\subsubsection{Lessons for Future Surveys}

For future versions of CBECS, EIA could consider incorporating portions of the methodology used in the recent California CEUS (CEC 2006) and adding the following variables for the next round of CBECS:

- ASHRAE climate zones. Use the system of climate zones in Standard 169-2006 (ASHRAE 2006) to organize the survey and report results.

- Mechanical ventilation. Develop questions that seek to improve understanding of the type of outdoor air systems in use, the HVAC equipment related to mechanical ventilation, and how the outdoor air system might be operated.

- Monthly demand and energy. Examine monthly billing data and compile monthly data about the energy and peak power for each building. These data are valuable for comparisons to simulation results because they help identify how weather affects energy performance.

- Building shape and orientation. Develop questions that seek to improve understanding of the overall shape and orientation of the building. Final data would include:

- The ratio between the length and width (aspect ratio)

○ An indication of the orientation of the long axis with respect to north

- The height of the building or the typical floor-to-floor height

- A roof shape and orientation type (flat, tilted, etc.; important for solar systems).

- Utility Tariffs. Examine billing data and compile data about the rate schedules for utilities. Either identify the utility company and schedule, or provide numeric data for the rate structure.

- Change CDD to base $50^{\circ} \mathbf{F}$. A base of $65^{\circ} \mathrm{F}$ is currently used for CBECS, but this is considered less appropriate than a base of $50^{\circ} \mathrm{F}$ for commercial buildings.

Many of the real-world data underlying building science and energy modeling are based on anecdotal studies of relatively small numbers of buildings. A well-coordinated, statistically robust survey of the stock that includes detailed on-site measurements would be invaluable to researchers and would help to improve simulation practices that are being increasingly used by analysts to rate the energy performance of buildings (e.g., for tax credits). An aggressive (and very costly) expansion of the scope of future survey efforts would be a significant aid to the buildings industry, and researchers. Entities engaged in future surveys of commercial buildings should consider the following activities (in order of priority):

1. Submeter end uses.

2. Measure fan system static pressures and efficiencies.

3. Develop energy modeling inputs during site visits.

4. Measure outdoor air change rates.

5. Measure lighting conditions (levels).

6. Measure thermal comfort conditions. 
7. Measure hot and cold water use.

8. Measure moisture performance (indoor relative humidity, equipment for humidification and/or dehumidification).

9. Measure air quality. 


\section{Conclusions and Recommendations}

A methodology for analyzing the energy performance of commercial buildings was developed and examined for its ability to represent the entire sector. This report documents how the method uses the building characteristic data for each sample in the 2003 CBECS public use data to create EnergyPlus models and how well the models agree with the energy consumption data from CBECS that were reserved for validation. We make the following conclusions:

- Bottom-up modeling of the commercial sector is doable and appears able to capture the breadth of variation in whole-building energy performance. The main goal of this study was to evaluate the methodology for modeling the sector, and we conclude that method is valid. The statistical sample of buildings from 2003 CBECS can be extrapolated to develop a large-N sector model based on a set of EnergyPlus models. This sector model can be used for subsequent research to assess energy performance in the commercial buildings sector.

- Using a large-N sector model requires considerable computing resources. Using 4,820 EnergyPlus models requires about 400 hours of simulation run time for each scenario (3gigahertz processors, 2-gigabyte random access memory). The method is not for the faint of heart and appears most applicable in a research setting with access to cluster computing. Experience suggests that smaller-N sector models (around 200) should be developed to reduce the computational burden of future analyses of the entire sector. The current method is suggested for producing reference data sets and for validating smaller models of the sector.

- EnergyPlus model results can be roughly consistent with 2003 CBECS. Individually, the models and survey sample building results do not agree well because there are insufficient data in CBECS to produce an accurate model. But when aggregating large numbers of models, the overall magnitudes and trends agree as well as can be expected. Although modeling tools are often used only in differential manner, the absolute values for predictions of annual fuel use and expenditures are encouraging. The model results often deviate from the survey, but the comparison to survey results provides a useful check against reality, and the $12 \%$ overall agreement in site EUI demonstrates much better than an "order of magnitude" validation of EnergyPlus model predictions.

- The model input and detail could be improved in many ways. One could use the survey results for energy consumption to continue to refine model input assignments. We used only the building characteristics data from 2003 CBECS to derive the models in the current study in an effort to validate such modeling. The current effort selected many details at random, but an additional effort could search unknown input parameters for those that agree with the survey energy results. There would still be insufficient data to model particular end uses without suitable end-use submetering.

- Though less robust than the whole-building data from 2003 CBECS, data for end uses from the Building Energy Data Book suggest that more research is needed to improve understanding of energy end uses. Research is needed to better understand the source of the differences in water heating, space cooling, and fans between this study and the Building Energy Data Book. This research should evaluate which one is more accurate and therefore help assess how effectively the methodology evaluated in this study can be used to evaluate the energy savings potential of end use-specific technologies and design approaches. 


\section{Bibliography}

ADL. (1993). Characterization of Commercial Building Appliances. Final report to the U.S. Department of Energy, June 1993.

ASHRAE. (1999). ANSI/ASHRAE/IESNA Standard 90.1-1999: Energy Standard for Buildings except Low-Rise Residential Buildings. Atlanta, GA: ASHRAE.

ASHRAE. (2001a). ANSI/ASHRAE/IESNA Standard 90.1-2001: Energy Standard for Buildings except Low-Rise Residential Buildings. Atlanta, GA: ASHRAE.

ASHRAE. (2001b). 2001 ASHRAE Handbook: Fundamentals. Chapter 29, Table 12, "Plug Loads." Atlanta, GA: ASHRAE.

ASHRAE. (2004a). ANSI/ASHRAE/IESNA Standard 90.1-2004: Energy Standard for Buildings except Low-Rise Residential Buildings. Atlanta, GA: ASHRAE.

ASHRAE. (2004b). Standard 140-2004 - Standard Method of Test for the Evaluation of Building Energy Analysis Computer Programs (ANSI Approved). Atlanta, GA: ASHRAE.

ASHRAE. (2003). 2003 ASHRAE Handbook: HVAC Applications. Atlanta, GA: ASHRAE.

ASHRAE. (2006). ANSI/ASHRAE Standard 169-2006: Weather Data for Building Design Standards. Atlanta, GA: ASHRAE.

Benne, K., Griffith, B.; Long, N.; Torcellini, P.; Crawley, D.; Logee, T. (2008). Assessment of the Energy Impacts of Outside Air in the Commercial Sector. In Draft. NREL Report No. TP-550-41955. Golden, CO: NREL.

Briggs, R.S.; Crawley, D.B.; Schliesing, J.S. (1992). Energy Requirements for Office Buildings, Volume 1, Existing Buildings. GRI-90/0236.1, February. Chicago: Gas Research Institute (GRI).

Briggs, R.S., Crawley, D.B.; Belzer, D.B. (1987). Analyses and Categorization of the Office Building Stock. GRI-87/0244, October. Chicago: GRI.

CEC. (2006). California Commercial End-Use Survey. March 2006. California Energy Commission. CEC-400-2006-005.

Chan, W.R. (2006). Assessing the Effectiveness of Shelter-in-Place as an Emergency Response to LargeScale Outdoor Chemical Releases. Dissertation. Department of Civil and Environmental Engineering, University of California, Berkeley. 2006. LBNL-60184.

Colliver, D.G. (1995). Energy Requirements for Conditioning of Ventilating Air. Technical Note AIVC 47. Coventry, UK: Air Infiltration and Ventilation Centre.

Crawley, D.B.; Schliesing, J.S. (1992). Energy Requirements for Office Buildings, Volume 2, Recent and Future Buildings. GRI-90/0236.2, March. Chicago: GRI.

Barley, D.; Deru, M.; Pless, S.; Torcellini, P. (2005). Procedure for Measuring and Reporting Commercial Building Energy Performance. Golden, CO: NREL/TP-550-38601.

Deru, M.; Torcellini, P. (2007). Source Energy and Emission Factors for Energy Use in Buildings. Golden, CO: NREL/TP-550-38617. Revised June 2007.

DOE. (2000). High-Performance Commercial Buildings: A Technology Roadmap. Washington, DC:

DOE. Available from www.eere.energy.gov/buildings/documents/pdfs/roadmap lowres.pdf.

DOE. (2002). U.S. Lighting Market Characterization Volume 1: National Lighting Inventory and Energy Consumption Estimate. Washington, DC: DOE. Available from www.eere.energy.gov/buildings/info/documents/pdfs/lmc_voll final.pdf. 
DOE. (2006). 2006 Buildings Energy Data Book. Washington, DC: DOE. Available from http://buildingsdatabook.eren.doe.gov/.

DOE. (2005a). DOE Solar Energy Technologies Program FY 2004 Annual Report. Washington, DC:

DOE. Available from www.osti.gov/bridge.

DOE. (2007). EnergyPlus Version 2.0. Computer program available at www.energyplus.gov

EIA. (1983). Nonresidential Buildings Energy Consumption Survey: 1979 Consumption and Expenditures, Part 1: Natural Gas and Electricity. DOE/EIA-0318/1. Washington, DC: EIA.

EIA. (1995). Commercial Buildings Consumption and Expenditures 1992. DOE/EIA-0318(92). Washington, DC: EIA.

EIA. (1998). A Look at Commercial Buildings in 1995: Characteristics, Energy Consumption, and Energy Expenditures. Washington, DC: EIA. Available from www.eia.doe.gov/emeu/cbecs/report_1995.html.

EIA. (2002). 1999 Commercial Buildings Energy Consumption Survey. Washington, DC: EIA. Available from www.eia.doe.gov/emeu/cbecs/1999publicuse/99microdat.html.

EIA. (2003). End-Use Consumption by Principal Building Activity, 1999 (Preliminary Estimates). Washington, DC: EIA. Available from www.eia.doe.gov/emeu/cbecs/enduse consumption/pba.html.

EIA. (2006). 2003 Commercial Buildings Energy Consumption Survey. Washington, DC: EIA. Available from www.eia.doe.gov/emeu/cbecs/cbecs2003/introduction.html.

EIA. (2007). Annual Energy Outlook 2007. Energy Information Administration Washington, DC: EIA. Available from www.eia.doe.gov/oiaf/aeo.

EPA. (2003). U.S. EPA eGRID2002 Version 2.01. May 2003. Washington, DC: EPA. www.epa.gov/cleanenergy/energy-resources/egrid/index.html.

Fisk, W.J. (2000). Health and Productivity Gains from Better Indoor Environments and Their Implications for the U.S. Department of Energy. Berkeley, CA: Lawrence Berkeley National Laboratory. LBNL-47458.

GARD Analytics. (2004a). EnergyPlus Testing with ANSI/ASHRAE Standard 140-2001 (BESTEST). EnergyPlus Version 1.2.0.029. Park Ridge, IL: GARD. Available from www.energyplus.gov.

GARD Analytics. (2004b). EnergyPlus Testing with HVAC BESTEST Part 1-Tests E100 to E200. EnergyPlus Version 1.2.0.029. Park Ridge, IL: GARD. Available from www.energyplus.gov.

Griffith, B.; Crawley, D. (2006). "Methodology for Analyzing the Technical Potential for Energy Performance in the U.S. Commercial Buildings Sector with Detailed Energy Modeling." Proceedings of SimBuild 2006 Conference, Cambridge, MA. NREL Report No. CP-550-40124. Golden, CO: NREL.

Griffith, B.; Long, N.; Torcellini, P.; Judkoff, R.; Crawley, D.; Ryan, J. (2007). Assessment of the Technical Potential for Achieving Net-Zero-Energy Buildings in the Commercial Sector. NREL Report No. TP-550-41957. Golden, CO: NREL.

Huang, J.; Franconi, E. (1999). Commercial Heating and Cooling Loads Component Analysis. Berkeley, CA: Lawrence Berkeley National Laboratory.

Judkoff, R.; Neymark, J. (1995). International Energy Agency Building Energy Simulation Test (BESTEST) and Diagnostic Method. NREL Report No. TP-472-6231. Golden, CO: NREL.

Litzkow, M.; Livny, M.; Mutka, M. (1998). "Condor-A Hunter of Idle Workstations." Proceedings of the 8th International Conference of Distributed Computing Systems. Washington, DC: IEEE; pp. 104111. 
Lomas, K.J.; Eppel, H. (1992). "Sensitivity Analysis Techniques for Building Thermal Simulation Programs." Energy and Buildings 19:21-44. Amsterdam: Elsevier Science.

MacDonald, I.A. (2002). Quantifying the Effects of Uncertainty in Building Simulation. Ph.D. Thesis. Glasgow, UK: University of Strathclyde.

Marion, W.; Wilcox, S. (1995). Solar Radiation Data Manual for Buildings. NREL/TP-463-7904. Golden, CO: NREL.

Moffat, S. (2001). Stock Aggregation: Methods for Evaluating the Environmental Performance of Building Stocks. Background report from the International Energy Agency's Annex 31 Energy-Related Environmental Impact of Buildings. Available from www.annex31.com/.

Neymark, J.; Judkoff, R. (2002). International Energy Agency Building Energy Simulation Test and Diagnostic Method for Heating, Ventilating, and Air-Conditioning Equipment Models (HVAC BESTEST); Volume 1: Cases E100-E200. NREL/TP-550-30152. Golden, CO: NREL.

NREL. (2007). National Solar Radiation Database 1991-2005 Update: Users Manual. NREL/TP-58141364. Golden, CO: NREL.

NREL. (2000). Photovoltaics -Energy for the New Millennium: The National Photovoltaics Program Plan, 2000-2004. (2000). 22 pp.; NREL/BR-520-25847; DOE/GO-10099-940.

Orme, M. (2001). "Estimates of the Energy Impact of Ventilation and Associated Financial Expenditures." Energy and Buildings 33:199-205. Amsterdam: Elsevier Science.

Persily, A.K. (1998). Airtightness of Commercial and Institutional Buildings: Blowing Holes in the Myth of Tight Buildings. DOE, ASHRAE, ORNL Conference - Thermal Envelopes VII, Clear Water, FL.

Reed, J.H.; Johnson, K.; Riggert, J.; Oh, A.D. (2004). Who Plays and Who Decides: The Structure and Operation of the Commercial Building Market. Rockville, MD: Innovologie LLC.

Roth, K.W.; Goldstein, F.; Kleinman, J. (2002). Energy Consumption by Office and Telecommunications Equipment in Commercial Buildings. Volume I: Energy Consumption Baseline. Cambridge, MA:

Arthur D. Little, Inc.

Roth, K.W.; Larocque, G.R.; Kleinman, J. (2004). Energy Consumption by Office and Telecommunications Equipment in Commercial Buildings. Volume II: Energy Savings Potential. Cambridge, MA: TIAX LLC.

Sachs, H.; Nadel, S.; Thorne Amann, J.; Tuazon, M.; Mendelsohn, E.; Rainer, L.; Todesco, G.; Shipley, D.; Adelaar, M. (2004). Emerging Energy-Saving Technologies and Practices for the Building Sector as of 2004. Washington, DC: American Council for an Energy-Efficient Economy.

Seppanen, O.A.; Fisk, W.J.; Mendell, M.J. (1999). "Association of Ventilation Rates and $\mathrm{CO}_{2}$ Concentrations with Health and Other Responses in Commercial and Institutional Buildings." Indoor Air pp. 226-252. Also LBNL-43334.

Taylor, J.R. (1982). An Introduction to Error Analysis. Mill Valley, CA: University Science Books.

TIAX. (2005). Energy Impacts of Commercial Building Controls and Performance Diagnostics: Market Characterization, Energy Impact of Building Faults and Energy Savings Potential. Final report prepared by TIAX LLC for the U.S. Department of Energy. Available at www.eere.energy.gov/buildings/highperformance/pdfs/tiax controls diagnostics.pdf.

TIAX. (2006). Commercial and Residential Sector Miscellaneous Electricity Consumption: FY2005 and Projections to 2030. Final report by TIAX LLC to the U.S. Department of Energy, Energy Information Adminstration (DOE/EIA), September 2006. 
Torcellini, P. (2001). “Better Buildings by Design.” Solar Today (March/April 2001).

Torcellini, P.; Long, N.; Judkoff, R. (2004). “Consumptive Water Use for U.S. Power Production." ASHRAE Transactions 2003 110:1. Atlanta, GA: ASHRAE. Also NREL/CP-550-35190.

Turk, B.H.; Grimsrud, D.T.; Brown, J.T.; Geisling-Sobotka, K.L.; Harrison, J.; Prill, R.J. (1989).

"Commercial Building Ventilation Rates and Particle Concentrations." ASHRAE Transactions 95:422433. Atlanta, GA: ASHRAE. 


\section{Appendix A. Expanded Results, Tables, and Figures}

This appendix contains ancillary results that augment results presented in the main body of the report.

\section{A.1 EnergyPlus Modeling Outcomes}

This subsection provides ancillary data about the modeling methods. Table A-1 provides summary data about the EnergyPlus simulation run times we observed. One issue involved with the chosen methodology is the computational burden associated with using so many EnergyPlus models. Each scenario or iteration involves running 4,820 models and requires roughly 15 days of processor time (3gigahertz processors). Scenarios also need to be repeated many times to iterate input data. Distributed computing was necessary to complete the work in a reasonable amount of calendar time by running multiple models at the same time on different processors.

Table A-1 EnergyPlus Modeling Simulation Run Times by Subsector

\begin{tabular}{|l|c|c|c|c|}
\hline \multirow{2}{*}{ Subsector (PBA) } & \multicolumn{2}{|c|}{ 2003 Historical Weather } & \multicolumn{2}{c|}{ TMY Weather } \\
\cline { 2 - 5 } & $\begin{array}{c}\text { Weighted } \\
\text { Mean } \\
\text { Run Time } \\
\text { (s) }\end{array}$ & $\begin{array}{c}\text { Weighted } \\
\text { Standard } \\
\text { Deviation } \\
\text { (s) }\end{array}$ & $\begin{array}{c}\text { Weighted } \\
\text { Mean Run } \\
\text { Time } \\
\text { (s) }\end{array}$ & $\begin{array}{c}\text { Weighted } \\
\text { Standard } \\
\text { Deviation } \\
\text { (s) }\end{array}$ \\
\hline \hline All & 210 & 148 & 218 & 148 \\
\hline Office/professional & 281 & 134 & 292 & 130 \\
\hline Nonrefrigerated warehouse & 97 & 77 & 105 & 76 \\
\hline Education & 218 & 125 & 226 & 126 \\
\hline Retail (except malls) & 148 & 97 & 155 & 96 \\
\hline Public assembly & 226 & 138 & 230 & 133 \\
\hline Service & 130 & 85 & 134 & 86 \\
\hline Religious worship & 203 & 113 & 201 & 106 \\
\hline Lodging & 337 & 208 & 357 & 203 \\
\hline Food services & 209 & 177 & 212 & 183 \\
\hline Inpatient health care & 364 & 140 & 397 & 127 \\
\hline Public order and safety & 234 & 126 & 246 & 129 \\
\hline Food sales & 125 & 69 & 137 & 72 \\
\hline Outpatient health care & 251 & 164 & 247 & 151 \\
\hline Vacant & 134 & 115 & 145 & 114 \\
\hline Other & 187 & 129 & 187 & 125 \\
\hline Skilled nursing & 265 & 152 & 270 & 152 \\
\hline Laboratory & 390 & 139 & 384 & 119 \\
\hline Refrigerated warehouse & 107 & 64 & 112 & 80 \\
\hline
\end{tabular}

The methods discussed in Section 2.5, Section C.16, and Section C.20 were used to assign outside air change rates; the results for outside air are summarized in Table A-2 by subsector and in Table A-3 by climate zone. Various input assumptions that define the rates of outside air in the modeling result in sector-wide air change rates of $0.8 \mathrm{ACH}$ with $66 \%$ from mechanical ventilation and $34 \%$ from infiltration. 
Table A-2 Average Occupied Outside Air Rates in Modeling by Subsector

\begin{tabular}{|c|c|c|c|c|c|c|c|}
\hline \multirow[b]{2}{*}{ Subsector (PBA) } & \multirow[b]{2}{*}{$\mathbf{N}$} & \multicolumn{2}{|c|}{$\begin{array}{l}\text { Mechanical } \\
\text { Ventilation }\end{array}$} & \multicolumn{2}{|c|}{ Infiltration } & \multicolumn{2}{|c|}{$\begin{array}{l}\text { Combined } \\
\text { Mechanical and } \\
\text { Infiltration }\end{array}$} \\
\hline & & $\begin{array}{c}\text { Weighted } \\
\text { Mean } \\
(\mathrm{ACH})\end{array}$ & $\begin{array}{c}\text { Weighted } \\
\text { Standard } \\
\text { Deviation } \\
\text { (ACH) }\end{array}$ & $\begin{array}{c}\text { Weighted } \\
\text { Mean } \\
(\mathrm{ACH})\end{array}$ & $\begin{array}{c}\text { Weighted } \\
\text { Standard } \\
\text { Deviation } \\
\text { (ACH) }\end{array}$ & $\begin{array}{l}\text { Weighted } \\
\text { Mean } \\
\text { (ACH) }\end{array}$ & $\begin{array}{c}\text { Weighted } \\
\text { Standard } \\
\text { Deviation } \\
\text { (ACH) }\end{array}$ \\
\hline All & 4,820 & 0.53 & 0.91 & 0.27 & 0.14 & 0.79 & 0.92 \\
\hline Office/professional & 976 & 0.52 & 0.41 & 0.27 & 0.14 & 0.79 & 0.39 \\
\hline $\begin{array}{l}\text { Nonrefrigerated } \\
\text { warehouse }\end{array}$ & 473 & 0.04 & 0.05 & 0.23 & 0.13 & 0.26 & 0.14 \\
\hline Education & 649 & 0.63 & 0.62 & 0.28 & 0.11 & 0.92 & 0.65 \\
\hline Retail (except malls) & 355 & 0.36 & 0.21 & 0.32 & 0.11 & 0.68 & 0.21 \\
\hline Public assembly & 279 & 0.46 & 0.68 & 0.26 & 0.11 & 0.72 & 0.70 \\
\hline Service & 370 & 0.11 & 0.00 & 0.33 & 0.00 & 0.44 & 0.20 \\
\hline Religious worship & 311 & 0.33 & 0.13 & 0.30 & 0.14 & 0.63 & 0.43 \\
\hline Lodging & 260 & 0.60 & 0.39 & 0.24 & 0.09 & 0.84 & 0.34 \\
\hline Food services & 242 & 2.79 & 0.36 & 0.42 & 0.11 & 3.21 & 2.85 \\
\hline Inpatient health care & 217 & 2.72 & 2.81 & 0.15 & 0.12 & 2.87 & 1.92 \\
\hline Public order and safety & 85 & 0.34 & 1.93 & 0.24 & 0.07 & 0.59 & 0.29 \\
\hline Food sales & 125 & 0.46 & 0.00 & 0.38 & 0.00 & 0.84 & 0.25 \\
\hline Outpatient health care & 144 & 0.82 & 0.25 & 0.35 & 0.14 & 1.16 & 0.54 \\
\hline Vacant & 134 & 0.04 & 0.25 & 0.04 & 0.11 & 0.08 & 0.24 \\
\hline Other & 64 & 0.11 & 0.57 & 0.25 & 0.12 & 0.36 & 0.17 \\
\hline Skilled nursing & 73 & 0.78 & 0.18 & 0.28 & 0.10 & 1.06 & 0.43 \\
\hline Laboratory & 43 & 1.47 & 0.15 & 0.18 & 0.12 & 1.65 & 0.90 \\
\hline Refrigerated warehouse & 20 & 0.07 & 0.44 & 0.24 & 0.10 & 0.30 & 0.11 \\
\hline
\end{tabular}


Table A-3 Average Occupied Outside Air Rates in Modeling by Climate Zone: 2003 Historical Weather

\begin{tabular}{|c|c|c|c|c|c|c|c|}
\hline \multirow{2}{*}{$\begin{array}{c}\text { Climate } \\
\text { Zone }\end{array}$} & \multirow[b]{2}{*}{$\mathbf{N}$} & \multicolumn{2}{|c|}{ Mechanical Ventilation } & \multicolumn{2}{|c|}{ Infiltration } & \multicolumn{2}{|c|}{$\begin{array}{l}\text { Combined } \\
\text { Mechanical and } \\
\text { Infiltration }\end{array}$} \\
\hline & & $\begin{array}{c}\text { Weighted } \\
\text { Mean (ACH) }\end{array}$ & $\begin{array}{c}\text { Weighted } \\
\text { Standard } \\
\text { Deviation } \\
\text { (ACH) }\end{array}$ & $\begin{array}{l}\text { Weighted } \\
\text { Mean } \\
\text { (ACH) }\end{array}$ & $\begin{array}{c}\text { Weighted } \\
\text { Standard } \\
\text { Deviation } \\
\text { (ACH) }\end{array}$ & $\begin{array}{l}\text { Weighted } \\
\text { Mean } \\
(\mathrm{ACH})\end{array}$ & $\begin{array}{c}\text { Weighted } \\
\text { Standard } \\
\text { Deviation } \\
\text { (ACH) }\end{array}$ \\
\hline All & 4,820 & 0.53 & 0.91 & 0.27 & 0.14 & 0.79 & 0.92 \\
\hline $1 \mathrm{~A}$ & 40 & 0.73 & 1.86 & 0.28 & 0.10 & 1.01 & 1.89 \\
\hline $2 A$ & 542 & 0.65 & 1.08 & 0.27 & 0.14 & 0.92 & 1.10 \\
\hline $2 B$ & 24 & 0.59 & 1.04 & 0.26 & 0.15 & 0.85 & 1.04 \\
\hline $3 A$ & 464 & 0.53 & 0.81 & 0.28 & 0.14 & 0.81 & 0.82 \\
\hline 3B & 452 & 0.65 & 1.18 & 0.27 & 0.14 & 0.92 & 1.20 \\
\hline $3 C$ & 52 & 0.75 & 0.67 & 0.19 & 0.13 & 0.94 & 0.69 \\
\hline $4 \mathrm{~A}$ & 982 & 0.49 & 0.77 & 0.26 & 0.13 & 0.75 & 0.78 \\
\hline $4 \mathrm{~B}$ & 64 & 0.64 & 0.74 & 0.26 & 0.17 & 0.90 & 0.75 \\
\hline $4 \mathrm{C}$ & 50 & 0.56 & 2.07 & 0.28 & 0.15 & 0.84 & 2.10 \\
\hline $5 A$ & 1,152 & 0.48 & 0.84 & 0.25 & 0.13 & 0.73 & 0.84 \\
\hline $5 B$ & 262 & 0.54 & 0.73 & 0.31 & 0.14 & 0.86 & 0.74 \\
\hline $6 \mathrm{~A}$ & 519 & 0.44 & 0.79 & 0.26 & 0.13 & 0.70 & 0.81 \\
\hline $6 \mathrm{~B}$ & 120 & 0.56 & 0.74 & 0.32 & 0.13 & 0.88 & 0.74 \\
\hline $7 \mathrm{~A}$ & 97 & 0.30 & 0.73 & 0.26 & 0.15 & 0.57 & 0.78 \\
\hline $8 \mathrm{~A}$ & 0 & $\mathrm{~N} / \mathrm{A}$ & $\mathrm{N} / \mathrm{A}$ & $\mathrm{N} / \mathrm{A}$ & N/A & $\mathrm{N} / \mathrm{A}$ & $\mathrm{N} / \mathrm{A}$ \\
\hline
\end{tabular}




\section{A.2 Table Data for Figures in Section 4}

This section provides tables of results that correspond to figures presented in Section 4.0. These data tables augment the plots and add weighted standard deviations and calculated percent differences.

Table A-4 Total EUI Results from 2003 CBECS Survey and Modeling by Subsector

\begin{tabular}{|c|c|c|c|c|c|c|}
\hline \multirow[b]{2}{*}{ Subsector (PBA) } & \multirow[b]{2}{*}{$\mathbf{N}$} & \multicolumn{2}{|c|}{$\begin{array}{c}2003 \\
\text { CBECS Survey }\end{array}$} & \multicolumn{2}{|c|}{$\begin{array}{c}\text { Models } \\
2003 \text { Historical Weather }\end{array}$} & \multirow{2}{*}{$\begin{array}{c}\% \\
\text { Difference } \\
\text { in Mean } \\
\text { EUI }\end{array}$} \\
\hline & & $\begin{array}{l}\text { Weighted } \\
\text { Mean EUI } \\
\text { (kBtu/ft'ㄹ.yr) }\end{array}$ & $\begin{array}{l}\text { Weighted } \\
\text { Standard } \\
\text { Deviation } \\
\left(\mathrm{kBtu}^{\prime} \mathrm{ft}^{2} \cdot \mathrm{yr}\right)\end{array}$ & $\begin{array}{l}\text { Weighted } \\
\text { Mean EUI } \\
\text { (kBtu/ft'․yr) }\end{array}$ & $\begin{array}{l}\text { Weighted } \\
\text { Standard } \\
\text { Deviation } \\
\left(\mathrm{kBtu}^{2} \mathrm{ft}^{2} \cdot \mathrm{yr}\right)\end{array}$ & \\
\hline All & 4,820 & 89.8 & 97.4 & 79.2 & 74.2 & $12 \%$ \\
\hline Office/professional & 976 & 92.9 & 68.1 & 78.5 & 34.0 & $16 \%$ \\
\hline $\begin{array}{l}\text { Nonrefrigerated } \\
\text { warehouse }\end{array}$ & 473 & 42.3 & 56.1 & 34.1 & 23.9 & $19 \%$ \\
\hline Education & 649 & 83.1 & 68.2 & 55.2 & 31.0 & $34 \%$ \\
\hline Retail (except malls) & 355 & 73.9 & 75.5 & 74.6 & 34.1 & $-1 \%$ \\
\hline Public assembly & 279 & 93.9 & 85.1 & 72.3 & 43.8 & $23 \%$ \\
\hline Service & 370 & 77.0 & 97.5 & 69.6 & 35.6 & $10 \%$ \\
\hline Religious worship & 311 & 43.5 & 34.4 & 53.0 & 42.6 & $-22 \%$ \\
\hline Lodging & 260 & 94.1 & 63.1 & 83.3 & 41.6 & $11 \%$ \\
\hline Food services & 242 & 258.3 & 232.5 & 347.0 & 124.3 & $-34 \%$ \\
\hline Inpatient health care & 217 & 249.2 & 126.0 & 180.5 & 99.9 & $28 \%$ \\
\hline Public order and safety & 85 & 115.8 & 56.9 & 80.3 & 40.6 & $31 \%$ \\
\hline Food sales & 125 & 199.7 & 122.1 & 200.5 & 53.5 & $0 \%$ \\
\hline Outpatient health care & 144 & 94.6 & 80.9 & 87.8 & 42.2 & $7 \%$ \\
\hline Vacant & 134 & 20.9 & 31.5 & 21.5 & 33.8 & $-3 \%$ \\
\hline Other & 64 & 79.4 & 72.8 & 62.4 & 25.9 & $21 \%$ \\
\hline Skilled nursing & 73 & 124.6 & 62.9 & 121.0 & 25.7 & $3 \%$ \\
\hline Laboratory & 43 & 305.4 & 169.6 & 329.7 & 64.5 & $-8 \%$ \\
\hline $\begin{array}{l}\text { Refrigerated } \\
\text { warehouse }\end{array}$ & 20 & 98.5 & 71.7 & 86.0 & 25.2 & $13 \%$ \\
\hline
\end{tabular}


Table A-5 Total EUI Results from CBECS Survey and Modeling by Climate Zone and Weather Data

\begin{tabular}{|c|c|c|c|c|c|c|c|}
\hline \multirow[b]{2}{*}{ Zone } & \multirow[b]{2}{*}{$\mathbf{N}$} & \multicolumn{2}{|c|}{2003 CBECS Survey } & \multicolumn{2}{|c|}{$\begin{array}{c}\text { Models } 2003 \\
\text { Historical Weather }\end{array}$} & \multicolumn{2}{|c|}{ Models TMY Weather } \\
\hline & & $\begin{array}{c}\text { Weighted } \\
\text { Mean EUI } \\
(\mathrm{kBtu} / \\
\left.\mathrm{ft}^{2} \cdot \mathrm{yr}\right)\end{array}$ & $\begin{array}{c}\text { Weighted } \\
\text { Standard } \\
\text { Deviation } \\
(\mathbf{k B t u} / \\
\left.\mathrm{ft}^{2} \cdot \mathrm{yr}\right)\end{array}$ & $\begin{array}{c}\text { Weighted } \\
\text { Mean EUI } \\
(\mathbf{k B t u} / \\
\left.\mathrm{ft}^{2} \cdot \mathrm{yr}\right)\end{array}$ & $\begin{array}{c}\% \\
\text { Difference } \\
\text { from } \\
\text { Survey in } \\
\text { Mean EUI }\end{array}$ & $\begin{array}{c}\text { Weighted } \\
\text { Mean EUI } \\
(\mathbf{k B t u} / \\
\left.\mathrm{ft}^{2} \cdot \mathrm{yr}\right)\end{array}$ & $\begin{array}{c}\% \\
\text { Difference } \\
\text { from } \\
\text { Survey in } \\
\text { Mean EUI }\end{array}$ \\
\hline All & 4,820 & 89.8 & 97.4 & 79.2 & $12 \%$ & 79.6 & $11 \%$ \\
\hline $1 \mathrm{~A}$ & 40 & 73.7 & 98.3 & 101.5 & $-38 \%$ & 97.7 & $-33 \%$ \\
\hline $2 A$ & 542 & 71.6 & 88.0 & 71.7 & $0 \%$ & 70.4 & $2 \%$ \\
\hline $2 B$ & 24 & 113.5 & 91.6 & 93.5 & $18 \%$ & 91.6 & $19 \%$ \\
\hline $3 A$ & 464 & 89.0 & 105.2 & 78.0 & $12 \%$ & 78.4 & $12 \%$ \\
\hline $3 B$ & 452 & 69.6 & 98.3 & 64.3 & $8 \%$ & 64.8 & $7 \%$ \\
\hline $3 C$ & 52 & 62.3 & 54.4 & 66.1 & $-6 \%$ & 67.0 & $-8 \%$ \\
\hline $4 \mathrm{~A}$ & 982 & 94.7 & 104.8 & 79.0 & $17 \%$ & 79.5 & $16 \%$ \\
\hline $4 \mathrm{~B}$ & 64 & 107.9 & 104.2 & 58.7 & $46 \%$ & 61.4 & $43 \%$ \\
\hline $4 \mathrm{C}$ & 50 & 98.6 & 89.4 & 66.8 & $32 \%$ & 70.8 & $28 \%$ \\
\hline $5 A$ & 1,152 & 104.4 & 99.0 & 86.4 & $17 \%$ & 86.4 & $17 \%$ \\
\hline $5 B$ & 262 & 86.8 & 85.3 & 74.7 & $14 \%$ & 76.9 & $11 \%$ \\
\hline $6 \mathrm{~A}$ & 519 & 89.4 & 89.8 & 85.7 & $4 \%$ & 86.6 & $3 \%$ \\
\hline $6 \mathrm{~B}$ & 120 & 96.6 & 73.2 & 87.0 & $10 \%$ & 91.6 & $5 \%$ \\
\hline $7 \mathrm{~A}$ & 97 & 71.0 & 70.5 & 91.5 & $-29 \%$ & 92.6 & $-30 \%$ \\
\hline $8 \mathrm{~A}$ & 0 & N/A & $\mathrm{N} / \mathrm{A}$ & $\mathrm{N} / \mathrm{A}$ & $\mathrm{N} / \mathrm{A}$ & $\mathrm{N} / \mathrm{A}$ & $\mathrm{N} / \mathrm{A}$ \\
\hline
\end{tabular}

Table A-6 Total EUI Results from CBECS Survey and Modeling by Census Division

\begin{tabular}{|c|c|c|c|c|c|c|}
\hline \multirow[b]{2}{*}{ Census Division } & \multirow[b]{2}{*}{$\mathbf{N}$} & \multicolumn{2}{|c|}{$\begin{array}{l}2003 \text { CBECS Survey } \\
\text { (except Malls) }\end{array}$} & \multicolumn{2}{|c|}{$\begin{array}{c}\text { Models } \\
2003 \text { Historical Weather }\end{array}$} & \multirow{2}{*}{$\begin{array}{c}\% \\
\text { Difference } \\
\text { in Mean } \\
\text { EUI }\end{array}$} \\
\hline & & $\begin{array}{c}\text { Weighted } \\
\text { Mean EUI } \\
\left(\mathrm{kBtu} / \mathrm{ft}^{2} \cdot \mathrm{yr}\right)\end{array}$ & $\begin{array}{l}\text { Weighted } \\
\text { Standard } \\
\text { Deviation } \\
\text { (kBtu/ft'ㄹ) }\end{array}$ & $\begin{array}{l}\text { Weighted } \\
\text { Mean EUI } \\
\left(\mathrm{kBtu} / \mathrm{ft}^{2} \cdot \mathrm{yr}\right)\end{array}$ & $\begin{array}{l}\text { Weighted } \\
\text { Standard } \\
\text { Deviation } \\
\text { (kBtu/ft'ㄹ) }\end{array}$ & \\
\hline All & 4,820 & 89.8 & 97.4 & 79.2 & 74.2 & $12 \%$ \\
\hline 1. New England & 195 & 99.0 & 109.9 & 93.0 & 86.5 & $6 \%$ \\
\hline 2. Middle Atlantic & 641 & 98.3 & 104.1 & 84.7 & 71.8 & $14 \%$ \\
\hline 3. East North Central & 860 & 108.1 & 99.0 & 82.7 & 69.1 & $23 \%$ \\
\hline 4. West North Central & 452 & 79.5 & 73.9 & 90.5 & 82.8 & $-14 \%$ \\
\hline 5. South Atlantic & 912 & 86.8 & 98.2 & 79.2 & 79.8 & $9 \%$ \\
\hline 6. East South Central & 279 & 91.1 & 114.5 & 74.1 & 62.7 & $19 \%$ \\
\hline 7. West South Central & 579 & 73.4 & 92.6 & 67.7 & 74.0 & $8 \%$ \\
\hline 8. Mountain & 305 & 103.8 & 86.9 & 78.1 & 52.4 & $25 \%$ \\
\hline 9. Pacific & 597 & 69.4 & 87.6 & 67.7 & 74.7 & $2 \%$ \\
\hline
\end{tabular}


Table A-7 Electricity EUI Results from 2003 CBECS Survey and Modeling by Subsector

\begin{tabular}{|c|c|c|c|c|c|c|}
\hline \multirow[b]{2}{*}{ Subsector (PBA) } & \multirow[t]{2}{*}{$\mathbf{N}$} & \multicolumn{2}{|c|}{2003 CBECS Survey } & \multicolumn{2}{|c|}{$\begin{array}{c}\text { Models } \\
2003 \text { Historical Weather }\end{array}$} & \multirow{2}{*}{$\begin{array}{c}\% \\
\text { Difference } \\
\text { in Mean } \\
\text { EUI }\end{array}$} \\
\hline & & $\begin{array}{l}\text { Weighted } \\
\text { Mean EUI } \\
\text { (kBtu/ft'․yr) }\end{array}$ & $\begin{array}{l}\text { Weighted } \\
\text { Standard } \\
\text { Deviation } \\
\left(\mathbf{k B t u} / \mathrm{ft}^{2} \cdot \mathrm{yr}\right)\end{array}$ & $\begin{array}{l}\text { Weighted } \\
\text { Mean EUI } \\
\text { (kBtu/ft't.yr) }\end{array}$ & $\begin{array}{l}\text { Weighted } \\
\text { Standard } \\
\text { Deviation } \\
\text { (kBtu/ft'ㄹ.yr) }\end{array}$ & \\
\hline All & 4,820 & 46.9 & 53.8 & 51.8 & 42.7 & $-10 \%$ \\
\hline Office/professional & 976 & 58.9 & 39.4 & 60.8 & 25.3 & $-3 \%$ \\
\hline $\begin{array}{l}\text { Nonrefrigerated } \\
\text { warehouse }\end{array}$ & 473 & 20.9 & 27.9 & 25.0 & 17.9 & $-19 \%$ \\
\hline Education & 649 & 37.6 & 29.7 & 34.9 & 16.2 & $7 \%$ \\
\hline Retail (except malls) & 355 & 48.8 & 56.8 & 54.4 & 22.6 & $-12 \%$ \\
\hline Public assembly & 279 & 42.5 & 57.1 & 40.2 & 25.1 & $6 \%$ \\
\hline Service & 370 & 36.9 & 39.4 & 47.5 & 26.2 & $-29 \%$ \\
\hline Religious worship & 311 & 16.6 & 14.9 & 25.2 & 17.4 & $-52 \%$ \\
\hline Lodging & 260 & 44.6 & 34.6 & 53.8 & 21.7 & $-21 \%$ \\
\hline Food services & 242 & 130.9 & 132.5 & 177.4 & 67.1 & $-36 \%$ \\
\hline Inpatient health care & 217 & 93.7 & 40.7 & 87.4 & 25.3 & $7 \%$ \\
\hline $\begin{array}{l}\text { Public order and } \\
\text { safety }\end{array}$ & 85 & 52.3 & 28.7 & 56.9 & 26.3 & $-9 \%$ \\
\hline Food sales & 125 & 166.1 & 101.5 & 158.5 & 38.0 & $5 \%$ \\
\hline Outpatient health & 144 & 55.0 & 40.4 & 55.8 & 24.3 & $-1 \%$ \\
\hline Vacant & 134 & 6.0 & 11.3 & 9.4 & 11.6 & $-57 \%$ \\
\hline Other & 64 & 42.3 & 50.5 & 47.8 & 21.1 & $-13 \%$ \\
\hline Skilled nursing & 73 & 52.4 & 30.1 & 87.0 & 21.1 & $-66 \%$ \\
\hline Laboratory & 43 & 133.1 & 62.6 & 207.0 & 59.4 & $-55 \%$ \\
\hline $\begin{array}{l}\text { Refrigerated } \\
\text { warehouse }\end{array}$ & 20 & 84.9 & 71.0 & 77.3 & 15.6 & $9 \%$ \\
\hline
\end{tabular}


Table A-8 Electricity EUI Results from CBECS Survey and Modeling by Climate Zone and Weather Data

\begin{tabular}{|c|c|c|c|c|c|c|c|}
\hline \multirow[b]{2}{*}{ Zone } & \multirow[b]{2}{*}{$\mathbf{N}$} & \multicolumn{2}{|c|}{2003 CBECS Survey } & \multicolumn{2}{|c|}{$\begin{array}{c}\text { Models } \\
2003 \text { Historical } \\
\text { Weather }\end{array}$} & \multicolumn{2}{|c|}{$\begin{array}{c}\text { Models } \\
\text { TMY Weather }\end{array}$} \\
\hline & & $\begin{array}{c}\text { Weighted } \\
\text { Mean EUI } \\
(\mathbf{k B t u} / \\
\left.\mathrm{ft}^{2} \cdot \mathbf{y r}\right)\end{array}$ & $\begin{array}{c}\text { Weighted } \\
\text { Standard } \\
\text { Deviation } \\
(\mathbf{k B t u} / \\
\left.\mathrm{ft}^{2} \cdot \mathrm{yr}\right)\end{array}$ & $\begin{array}{c}\text { Weighted } \\
\text { Mean EUI } \\
(\mathrm{kBtu} / \\
\left.\mathrm{ft}^{2} \cdot \mathrm{yr}\right)\end{array}$ & $\begin{array}{c}\% \\
\text { Difference } \\
\text { from } \\
\text { Survey in } \\
\text { Mean EUI }\end{array}$ & $\begin{array}{c}\text { Weighted } \\
\text { Mean EUI } \\
(\mathrm{kBtu} / \\
\left.\mathrm{ft}^{2} \cdot \mathrm{yr}\right)\end{array}$ & $\begin{array}{c}\% \\
\text { Difference } \\
\text { from } \\
\text { Survey in } \\
\text { Mean EUI }\end{array}$ \\
\hline All & 4,820 & 46.9 & 53.8 & 51.8 & $-10 \%$ & 51.6 & $-10 \%$ \\
\hline $1 \mathrm{~A}$ & 40 & 60.9 & 70.6 & 78.5 & $-29 \%$ & 75.8 & $-24 \%$ \\
\hline $2 \mathrm{~A}$ & 542 & 54.6 & 65.0 & 55.4 & $-2 \%$ & 54.5 & $0 \%$ \\
\hline $2 B$ & 24 & 95.8 & 54.1 & 63.1 & $34 \%$ & 62.1 & $35 \%$ \\
\hline $3 \mathrm{~A}$ & 464 & 55.5 & 66.3 & 58.6 & $-6 \%$ & 58.5 & $-5 \%$ \\
\hline $3 B$ & 452 & 44.2 & 48.5 & 50.7 & $-15 \%$ & 50.2 & $-14 \%$ \\
\hline $3 C$ & 52 & 35.3 & 28.2 & 51.8 & $-47 \%$ & 51.2 & $-45 \%$ \\
\hline $4 \mathrm{~A}$ & 982 & 49.0 & 53.3 & 54.1 & $-10 \%$ & 54.2 & $-11 \%$ \\
\hline 4B & 64 & 57.0 & 53.9 & 43.2 & $24 \%$ & 43.2 & $24 \%$ \\
\hline $4 \mathrm{C}$ & 50 & 60.8 & 86.2 & 50.2 & $18 \%$ & 49.8 & $18 \%$ \\
\hline $5 A$ & 1,152 & 44.6 & 50.2 & 50.4 & $-13 \%$ & 50.4 & $-13 \%$ \\
\hline $5 B$ & 262 & 48.3 & 50.0 & 48.1 & $0 \%$ & 47.7 & $1 \%$ \\
\hline $6 \mathrm{~A}$ & 519 & 34.2 & 40.1 & 43.4 & $-27 \%$ & 43.3 & $-27 \%$ \\
\hline $6 \mathrm{~B}$ & 120 & 35.0 & 33.2 & 45.4 & $-30 \%$ & 45.1 & $-29 \%$ \\
\hline $7 \mathrm{~A}$ & 97 & 34.0 & 41.5 & 46.5 & $-37 \%$ & 46.3 & $-36 \%$ \\
\hline $8 A$ & 0 & $\mathrm{~N} / \mathrm{A}$ & $\mathrm{N} / \mathrm{A}$ & $\mathrm{N} / \mathrm{A}$ & $\mathrm{N} / \mathrm{A}$ & $\mathrm{N} / \mathrm{A}$ & $\mathrm{N} / \mathrm{A}$ \\
\hline
\end{tabular}


Table A-9 Electricity EUI Results from CBECS Survey and Modeling by Census Division

\begin{tabular}{|c|c|c|c|c|c|c|}
\hline \multirow[b]{2}{*}{ Census Division } & \multirow[b]{2}{*}{$\mathbf{N}$} & \multicolumn{2}{|c|}{2003 CBECS Survey } & \multicolumn{2}{|c|}{$\begin{array}{c}\text { Models } \\
2003 \text { Historical Weather }\end{array}$} & \multirow{2}{*}{$\begin{array}{c}\% \\
\text { Difference } \\
\text { in Mean } \\
\text { EUI }\end{array}$} \\
\hline & & $\begin{array}{l}\text { Weighted } \\
\text { Mean EUI } \\
\text { (kBtu/ft'․yr) }\end{array}$ & 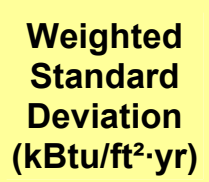 & $\begin{array}{l}\text { Weighted } \\
\text { Mean EUI } \\
(\text { kBtu/ft'․yr) }\end{array}$ & $\begin{array}{l}\text { Weighted } \\
\text { Standard } \\
\text { Deviation } \\
\left(\mathrm{kBtu} / \mathrm{ft}^{2} \cdot \mathrm{yr}\right)\end{array}$ & \\
\hline All & 4,820 & 46.9 & 53.8 & 51.8 & 42.7 & $-10 \%$ \\
\hline 1. New England & 195 & 36.5 & 49.8 & 52.0 & 53.8 & $-43 \%$ \\
\hline 2. Middle Atlantic & 641 & 39.7 & 47.5 & 51.3 & 36.3 & $-29 \%$ \\
\hline 3. East North Central & 860 & 45.1 & 50.2 & 46.6 & 37.1 & $-3 \%$ \\
\hline 4. West North Central & 452 & 38.8 & 40.8 & 50.2 & 39.3 & $-29 \%$ \\
\hline 5. South Atlantic & 912 & 58.5 & 66.3 & 59.2 & 49.4 & $-1 \%$ \\
\hline 6. East South Central & 279 & 50.3 & 58.8 & 53.2 & 40.4 & $-6 \%$ \\
\hline 7. West South Central & 579 & 49.7 & 55.4 & 52.3 & 49.0 & $-5 \%$ \\
\hline 8. Mountain & 305 & 51.9 & 54.0 & 47.2 & 29.6 & $9 \%$ \\
\hline 9. Pacific & 597 & 43.0 & 45.5 & 50.6 & 42.5 & $-18 \%$ \\
\hline
\end{tabular}


Table A-10 Natural Gas EUI Results from 2003 CBECS Survey and Modeling by Subsector

\begin{tabular}{|c|c|c|c|c|c|c|}
\hline \multirow[b]{2}{*}{ Subsector (PBA) } & \multirow[b]{2}{*}{$\mathbf{N}$} & \multicolumn{2}{|c|}{2003 CBECS Survey } & \multicolumn{2}{|c|}{$\begin{array}{c}\text { Models } \\
2003 \text { Historical Weather }\end{array}$} & \multirow{2}{*}{$\begin{array}{c}\% \\
\text { Difference } \\
\text { in Mean } \\
\text { EUI }\end{array}$} \\
\hline & & $\begin{array}{l}\text { Weighted } \\
\text { Mean Gas } \\
\text { EUI } \\
\left(k_{B} \text { tu/ft'tyr) }\right.\end{array}$ & $\begin{array}{l}\text { Weighted } \\
\text { Standard } \\
\text { Deviation } \\
\text { (kBtu/ft'ㄹ.yr) }\end{array}$ & $\begin{array}{c}\text { Weighted } \\
\text { Mean Gas } \\
\text { EUI } \\
\text { (kBtu/ft'ㄹr) }\end{array}$ & $\begin{array}{l}\text { Weighted } \\
\text { Standard } \\
\text { Deviation } \\
\left(\mathrm{kBtu}^{2} \mathrm{ft}^{2} \cdot \mathrm{yr}\right)\end{array}$ & \\
\hline All & 4,820 & 29.8 & 53.8 & 23.1 & 40.7 & $23 \%$ \\
\hline Office/professional & 976 & 22.1 & 40.6 & 14.2 & 20.8 & $36 \%$ \\
\hline $\begin{array}{l}\text { Nonrefrigerated } \\
\text { warehouse }\end{array}$ & 473 & 13.4 & 25.3 & 7.7 & 13.6 & $42 \%$ \\
\hline Education & 649 & 27.2 & 35.5 & 14.7 & 18.9 & $46 \%$ \\
\hline Retail (except malls) & 355 & 21.2 & 38.9 & 19.9 & 24.6 & $6 \%$ \\
\hline Public assembly & 279 & 25.9 & 40.2 & 22.9 & 33.6 & $12 \%$ \\
\hline Service & 370 & 34.4 & 75.2 & 21.9 & 23.1 & $36 \%$ \\
\hline Religious worship & 311 & 21.9 & 31.0 & 27.7 & 36.0 & $-27 \%$ \\
\hline Lodging & 260 & 36.9 & 41.5 & 19.3 & 23.5 & $48 \%$ \\
\hline Food services & 242 & 122.9 & 137.2 & 164.5 & 85.1 & $-34 \%$ \\
\hline Inpatient health care & 217 & 107.3 & 84.3 & 68.2 & 89.5 & $36 \%$ \\
\hline $\begin{array}{l}\text { Public order and } \\
\text { safety }\end{array}$ & 85 & 26.3 & 39.2 & 16.6 & 26.9 & $37 \%$ \\
\hline Food sales & 125 & 30.8 & 45.6 & 40.8 & 35.2 & $-32 \%$ \\
\hline Outpatient health care & 144 & 30.4 & 47.7 & 27.8 & 30.3 & $8 \%$ \\
\hline Vacant & 134 & 10.9 & 20.3 & 10.5 & 25.6 & $4 \%$ \\
\hline Other & 64 & 34.8 & 44.6 & 14.6 & 18.9 & $58 \%$ \\
\hline Skilled nursing & 73 & 63.8 & 49.8 & 31.8 & 23.3 & $50 \%$ \\
\hline Laboratory & 43 & 75.6 & 135.9 & 105.2 & 24.2 & $-39 \%$ \\
\hline $\begin{array}{l}\text { Refrigerated } \\
\text { warehouse }\end{array}$ & 20 & 9.2 & 10.4 & 7.7 & 20.5 & $16 \%$ \\
\hline
\end{tabular}


Table A-11 Natural Gas EUI Results from CBECS Survey and Modeling by Climate Zone and Weather Data

\begin{tabular}{|c|c|c|c|c|c|c|c|}
\hline \multirow[b]{2}{*}{ Zone } & \multirow[b]{2}{*}{$\mathbf{N}$} & \multicolumn{2}{|c|}{2003 CBECS Survey } & \multicolumn{2}{|c|}{$\begin{array}{c}\text { Models } \\
2003 \text { Historical Weather }\end{array}$} & \multicolumn{2}{|c|}{$\begin{array}{c}\text { Models } \\
\text { TMY Weather }\end{array}$} \\
\hline & & $\begin{array}{c}\text { Weighted } \\
\text { Mean EUI } \\
(\mathbf{k B t u} / \\
\left.\mathrm{ft}^{2} \cdot \mathrm{yr}\right)\end{array}$ & $\begin{array}{c}\text { Weighted } \\
\text { Standard } \\
\text { Deviation } \\
\text { (kBtu/ } \\
\left.\text { ft }^{2} \cdot \mathrm{yr}\right)\end{array}$ & $\begin{array}{c}\text { Weighted } \\
\text { Mean EUI } \\
(\text { kBtu/ } \\
\left.\mathrm{ft}^{2} \cdot \mathrm{yr}\right)\end{array}$ & $\begin{array}{c}\% \\
\text { Difference } \\
\text { from } \\
\text { Survey in } \\
\text { Mean EUI }\end{array}$ & $\begin{array}{c}\text { Weighted } \\
\text { Mean EUI } \\
(\mathrm{kBtu} / \\
\left.\mathrm{ft}^{2} \cdot \mathrm{yr}\right)\end{array}$ & $\begin{array}{c}\% \\
\text { Difference } \\
\text { from } \\
\text { Survey in } \\
\text { Mean EUI }\end{array}$ \\
\hline All & 4,820 & 29.8 & 53.8 & 23.1 & $23 \%$ & 23.8 & $20 \%$ \\
\hline $1 \mathrm{~A}$ & 40 & 10.4 & 39.4 & 13.4 & $-29 \%$ & 13.4 & $-29 \%$ \\
\hline $2 \mathrm{~A}$ & 542 & 10.2 & 29.2 & 10.0 & $2 \%$ & 10.3 & $-1 \%$ \\
\hline $2 B$ & 24 & 10.0 & 40.2 & 8.1 & $19 \%$ & 9.1 & $9 \%$ \\
\hline $3 \mathrm{~A}$ & 464 & 26.3 & 50.9 & 18.4 & $30 \%$ & 18.9 & $28 \%$ \\
\hline $3 B$ & 452 & 22.3 & 59.7 & 12.5 & $44 \%$ & 13.4 & $40 \%$ \\
\hline $3 \mathrm{C}$ & 52 & 20.6 & 39.9 & 14.3 & $31 \%$ & 15.6 & $24 \%$ \\
\hline $4 \mathrm{~A}$ & 982 & 28.2 & 57.0 & 18.4 & $35 \%$ & 18.9 & $33 \%$ \\
\hline $4 \mathrm{~B}$ & 64 & 48.3 & 58.4 & 14.5 & $70 \%$ & 17.2 & $64 \%$ \\
\hline $4 \mathrm{C}$ & 50 & 16.0 & 26.9 & 13.4 & $16 \%$ & 17.5 & $-10 \%$ \\
\hline $5 A$ & 1,152 & 39.7 & 56.1 & 31.5 & $21 \%$ & 31.6 & $20 \%$ \\
\hline $5 B$ & 262 & 32.6 & 51.7 & 24.9 & $24 \%$ & 27.5 & $16 \%$ \\
\hline $6 A$ & 519 & 38.6 & 57.5 & 37.4 & $3 \%$ & 38.3 & $1 \%$ \\
\hline $6 \mathrm{~B}$ & 120 & 52.9 & 55.3 & 38.8 & $27 \%$ & 43.6 & $18 \%$ \\
\hline $7 \mathrm{~A}$ & 97 & 24.8 & 38.5 & 43.6 & $-76 \%$ & 44.9 & $-81 \%$ \\
\hline $8 \mathrm{~A}$ & 0 & N/A & $\mathrm{N} / \mathrm{A}$ & $\mathrm{N} / \mathrm{A}$ & $\mathrm{N} / \mathrm{A}$ & $\mathrm{N} / \mathrm{A}$ & $\mathrm{N} / \mathrm{A}$ \\
\hline
\end{tabular}


Table A-12 Natural Gas EUI Results from CBECS Survey and Modeling by Census Division

\begin{tabular}{|c|c|c|c|c|c|c|}
\hline \multirow[b]{2}{*}{ Census Division } & \multirow[b]{2}{*}{$\mathbf{N}$} & \multicolumn{2}{|c|}{2003 CBECS Survey } & \multicolumn{2}{|c|}{$\begin{array}{c}\text { Models } \\
2003 \text { Historical Weather }\end{array}$} & \multirow{2}{*}{$\begin{array}{c}\% \\
\text { Difference } \\
\text { in Mean } \\
\text { EUI }\end{array}$} \\
\hline & & 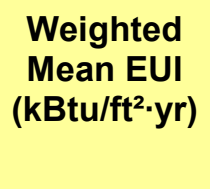 & $\begin{array}{l}\text { Weighted } \\
\text { Standard } \\
\text { Deviation } \\
\left(\mathrm{kBtu} / \mathrm{ft}^{2} \cdot \mathrm{yr}\right)\end{array}$ & $\begin{array}{l}\text { Weighted } \\
\text { Mean EUI } \\
\left(\mathrm{kBtu} / \mathrm{ft}^{2} \cdot \mathrm{yr}\right)\end{array}$ & $\begin{array}{l}\text { Weighted } \\
\text { Standard } \\
\text { Deviation } \\
\left(\mathbf{k B t u} / \mathrm{ft}^{2} \cdot \mathbf{y r}\right)\end{array}$ & \\
\hline All & 4,820 & 29.8 & 53.8 & 23.1 & 40.7 & $23 \%$ \\
\hline 1. New England & 195 & 25.2 & 50.2 & 34.2 & 40.4 & $-36 \%$ \\
\hline 2. Middle Atlantic & 641 & 35.5 & 62.6 & 24.7 & 47.7 & $30 \%$ \\
\hline 3. East North Central & 860 & 45.5 & 59.4 & 31.8 & 42.2 & $30 \%$ \\
\hline 4. West North Central & 452 & 32.2 & 43.8 & 38.3 & 52.6 & $-19 \%$ \\
\hline 5. South Atlantic & 912 & 17.7 & 40.7 & 15.4 & 35.0 & $13 \%$ \\
\hline 6. East South Central & 279 & 30.0 & 62.5 & 18.2 & 29.1 & $39 \%$ \\
\hline 7. West South Central & 579 & 19.9 & 46.1 & 13.2 & 30.6 & $34 \%$ \\
\hline 8. Mountain & 305 & 45.4 & 54.5 & 27.5 & 32.5 & $39 \%$ \\
\hline 9. Pacific & 597 & 20.1 & 53.0 & 14.9 & 36.8 & $26 \%$ \\
\hline
\end{tabular}


Table A-13 Total Energy Cost Intensity Results from 2003 CBECS Survey and Modeling by Subsector

\begin{tabular}{|c|c|c|c|c|c|c|}
\hline \multirow[b]{2}{*}{ Subsector (PBA) } & \multirow[b]{2}{*}{$\mathbf{N}$} & \multicolumn{2}{|c|}{2003 CBECS Survey } & \multicolumn{2}{|c|}{$\begin{array}{c}\text { Models } \\
2003 \text { Historical Weather }\end{array}$} & \multirow{2}{*}{$\begin{array}{c}\% \\
\text { Difference } \\
\text { in Mean } \\
\text { Cost } \\
\text { Intensity }\end{array}$} \\
\hline & & $\begin{array}{l}\text { Weighted } \\
\text { Mean Cost } \\
\text { Intensity } \\
\left(\$ / \mathrm{ft}^{2} \cdot \mathrm{yr}\right)\end{array}$ & $\begin{array}{c}\text { Weighted } \\
\text { Standard } \\
\text { Deviation } \\
\left(\$ / \mathrm{ft}^{2} \cdot \mathrm{yr}\right)\end{array}$ & $\begin{array}{l}\text { Weighted } \\
\text { Mean Cost } \\
\text { Intensity } \\
\left(\$ / \mathrm{ft}^{2} \cdot \mathrm{yr}\right)\end{array}$ & $\begin{array}{c}\text { Weighted } \\
\text { Standard } \\
\text { Deviation } \\
\left(\$ / \mathrm{ft}^{2} \cdot \mathrm{yr}\right)\end{array}$ & \\
\hline All & 4,820 & 1.51 & 1.62 & 1.63 & 1.44 & $-8 \%$ \\
\hline Office/professional & 976 & 1.80 & 1.17 & 1.90 & 0.95 & $-6 \%$ \\
\hline $\begin{array}{l}\text { Nonrefrigerated } \\
\text { warehouse }\end{array}$ & 473 & 0.68 & 0.71 & 0.76 & 0.55 & $-11 \%$ \\
\hline Education & 649 & 1.28 & 1.27 & 1.15 & 0.59 & $10 \%$ \\
\hline Retail (except malls) & 355 & 1.46 & 1.38 & 1.70 & 0.79 & $-17 \%$ \\
\hline Public assembly & 279 & 1.55 & 1.82 & 1.38 & 0.92 & $11 \%$ \\
\hline Service & 370 & 1.24 & 1.28 & 1.44 & 0.76 & $-16 \%$ \\
\hline Religious worship & 311 & 0.69 & 0.44 & 1.08 & 0.77 & $-56 \%$ \\
\hline Lodging & 260 & 1.51 & 1.09 & 1.65 & 0.95 & $-9 \%$ \\
\hline Food services & 242 & 4.38 & 4.02 & 6.25 & 2.85 & $-43 \%$ \\
\hline Inpatient health care & 217 & 2.95 & 1.39 & 2.75 & 1.55 & $7 \%$ \\
\hline Public order and safety & 85 & 1.86 & 0.82 & 1.65 & 0.87 & $11 \%$ \\
\hline Food sales & 125 & 4.20 & 2.82 & 4.31 & 1.84 & $-3 \%$ \\
\hline Outpatient health care & 144 & 1.77 & 1.25 & 1.87 & 0.95 & $-6 \%$ \\
\hline Vacant & 134 & 0.31 & 0.43 & 0.37 & 0.46 & $-19 \%$ \\
\hline Other & 64 & 1.52 & 1.82 & 1.68 & 0.94 & $-10 \%$ \\
\hline Skilled nursing & 73 & 1.68 & 0.89 & 2.27 & 0.85 & $-35 \%$ \\
\hline Laboratory & 43 & 4.61 & 2.33 & 5.54 & 2.20 & $-20 \%$ \\
\hline $\begin{array}{l}\text { Refrigerated } \\
\text { warehouse }\end{array}$ & 20 & 1.54 & 1.12 & 1.69 & 0.69 & $-10 \%$ \\
\hline
\end{tabular}


Table A-14 Total Energy Cost Intensity Results from CBECS Survey and Modeling by Climate Zone and Weather Data

\begin{tabular}{|c|c|c|c|c|c|c|c|}
\hline \multirow[b]{2}{*}{ Zone } & \multirow[b]{2}{*}{$\mathbf{N}$} & \multicolumn{2}{|c|}{2003 CBECS Survey } & \multicolumn{2}{|c|}{$\begin{array}{c}\text { Models } \\
2003 \text { Historical Weather }\end{array}$} & \multicolumn{2}{|c|}{$\begin{array}{l}\text { Models } \\
\text { TMY Weather }\end{array}$} \\
\hline & & $\begin{array}{c}\text { Weighted } \\
\text { Mean Cost } \\
\text { Intensity } \\
\left(\$ / \mathrm{ft}^{2} \cdot \mathrm{yr}\right)\end{array}$ & $\begin{array}{c}\text { Weighted } \\
\text { Standard } \\
\text { Deviation } \\
\left(\$ / \mathrm{ft}^{2} \cdot \mathrm{yr}\right)\end{array}$ & $\begin{array}{c}\text { Weighted } \\
\text { Mean Cost } \\
\text { Intensity } \\
\left(\$ / \mathrm{ft}^{2} \cdot \mathrm{yr}\right)\end{array}$ & $\begin{array}{c}\% \text { Difference } \\
\text { from Survey } \\
\text { in Mean Cost } \\
\text { Intensity }\end{array}$ & $\begin{array}{l}\text { Weighted } \\
\text { Mean Cost } \\
\text { Intensity } \\
\left(\$ / \mathrm{ft}^{2} \cdot \mathrm{yr}\right)\end{array}$ & $\begin{array}{c}\% \text { Difference } \\
\text { from Survey } \\
\text { in Mean Cost } \\
\text { Intensity }\end{array}$ \\
\hline All & 4,820 & 1.51 & 1.62 & 1.72 & $-14 \%$ & 1.71 & $-13 \%$ \\
\hline $1 \mathrm{~A}$ & 40 & 2.33 & 2.62 & 2.22 & $5 \%$ & 2.16 & $7 \%$ \\
\hline $2 \mathrm{~A}$ & 542 & 1.42 & 1.60 & 1.62 & $-14 \%$ & 1.60 & $-13 \%$ \\
\hline $2 B$ & 24 & 2.21 & 1.30 & 1.68 & $24 \%$ & 1.69 & $24 \%$ \\
\hline $3 \mathrm{~A}$ & 464 & 1.41 & 1.53 & 1.65 & $-17 \%$ & 1.65 & $-17 \%$ \\
\hline $3 B$ & 452 & 1.65 & 1.84 & 2.02 & $-22 \%$ & 2.00 & $-21 \%$ \\
\hline $3 C$ & 52 & 1.77 & 1.20 & 2.40 & $-36 \%$ & 2.39 & $-35 \%$ \\
\hline $4 \mathrm{~A}$ & 982 & 1.57 & 1.72 & 1.81 & $-15 \%$ & 1.80 & $-15 \%$ \\
\hline $4 \mathrm{~B}$ & 64 & 1.75 & 1.79 & 1.53 & $13 \%$ & 1.56 & $11 \%$ \\
\hline $4 \mathrm{C}$ & 50 & 2.21 & 3.93 & 1.12 & $49 \%$ & 1.14 & $48 \%$ \\
\hline $5 \mathrm{~A}$ & 1,152 & 1.57 & 1.49 & 1.79 & $-14 \%$ & 1.79 & $-14 \%$ \\
\hline $5 B$ & 262 & 1.32 & 1.52 & 1.18 & $11 \%$ & 1.19 & $10 \%$ \\
\hline $6 \mathrm{~A}$ & 519 & 1.30 & 1.35 & 1.55 & $-19 \%$ & 1.54 & $-18 \%$ \\
\hline $6 B$ & 120 & 1.24 & 1.04 & 1.09 & $12 \%$ & 1.11 & $10 \%$ \\
\hline $7 \mathrm{~A}$ & 97 & 0.96 & 0.98 & 1.68 & $-75 \%$ & 1.65 & $-72 \%$ \\
\hline $8 \mathrm{~A}$ & 0 & $\mathrm{~N} / \mathrm{A}$ & N/A & $\mathrm{N} / \mathrm{A}$ & $\mathrm{N} / \mathrm{A}$ & $\mathrm{N} / \mathrm{A}$ & $\mathrm{N} / \mathrm{A}$ \\
\hline
\end{tabular}

Table A-15 Total Energy Cost Intensity Results from CBECS Survey and Modeling by Census Division

\begin{tabular}{|c|c|c|c|c|c|c|}
\hline \multirow[b]{2}{*}{ Census Division } & \multirow[b]{2}{*}{$\mathbf{N}$} & \multicolumn{2}{|c|}{$\begin{array}{l}2003 \text { CBECS Survey } \\
\text { (except Malls) }\end{array}$} & \multicolumn{2}{|c|}{$\begin{array}{c}\text { Models } \\
2003 \text { Historical Weather }\end{array}$} & \multirow{2}{*}{$\begin{array}{c}\% \\
\text { Difference } \\
\text { in Mean } \\
\text { Cost } \\
\text { Intensity }\end{array}$} \\
\hline & & $\begin{array}{l}\text { Weighted } \\
\text { Mean Cost } \\
\text { Intensity } \\
\left(\$ / \mathrm{ft}^{2} \cdot \mathrm{yr}\right)\end{array}$ & $\begin{array}{c}\text { Weighted } \\
\text { Standard } \\
\text { Deviation } \\
\left(\$ / \mathrm{ft}^{2} \cdot \mathrm{yr}\right)\end{array}$ & $\begin{array}{l}\text { Weighted } \\
\text { Mean Cost } \\
\text { Intensity } \\
\left(\$ / \mathrm{ft}^{2} \cdot \mathrm{yr}\right)\end{array}$ & $\begin{array}{c}\text { Weighted } \\
\text { Standard } \\
\text { Deviation } \\
\left(\$ / \mathrm{ft}^{2} \cdot \mathrm{yr}\right)\end{array}$ & \\
\hline All & 4,820 & 1.51 & 1.62 & 1.72 & 1.33 & $-14 \%$ \\
\hline 1. New England & 195 & 1.73 & 1.89 & 2.14 & 1.65 & $-24 \%$ \\
\hline 2. Middle Atlantic & 641 & 1.75 & 1.93 & 2.43 & 1.58 & $-39 \%$ \\
\hline 3. East North Central & 860 & 1.45 & 1.29 & 1.56 & 1.10 & $-8 \%$ \\
\hline 4. West North Central & 452 & 1.08 & 0.97 & 1.28 & 0.85 & $-19 \%$ \\
\hline 5. South Atlantic & 912 & 1.51 & 1.58 & 1.54 & 1.15 & $-2 \%$ \\
\hline 6. East South Central & 279 & 1.42 & 1.59 & 1.39 & 0.99 & $2 \%$ \\
\hline 7. West South Central & 579 & 1.27 & 1.44 & 1.73 & 1.43 & $-36 \%$ \\
\hline 8. Mountain & 305 & 1.62 & 1.60 & 1.15 & 0.71 & $29 \%$ \\
\hline 9. Pacific & 597 & 1.73 & 2.03 & 1.87 & 1.53 & $-8 \%$ \\
\hline
\end{tabular}




\section{A.3 Probability Distributions}

The U.S. commercial sector is large and diverse, and its buildings have a wide variety of activities, sizes, shapes, locations, etc., that lead to wide variation in EUI. The previous section focused on the average results (weighted means) for entire portions of the commercial sector, but this should not be taken to imply that there is much uniformity in the energy performance of buildings. The large-N sector model used in this study allows us to examine variations in EUI across the sector. This section presents the distribution in results for a fuller understanding of how performance varies across the sector.

The plots in Figure A-1 to Figure A-20 present probability density functions (PDFs) that show how EUIs for all types of buildings are distributed for each climate zone. The plots are all normalized PDFs for how EUI is distributed in the commercial sector. PDFs are calculated from the 2003 CBECS public use data (survey) and from the EnergyPlus models with 2003 historical weather (models). Figure A-1 is for all the buildings the study. The remaining figures provide thumbnails of PDF plots to illustrate results by PBA and by climate zone. Each figure covers a PBA category and contains a group of plots for different climate zones. The upper left plot shows results for all climates taken together. The remaining 14 plots show results that are sorted by the individual ASHRAE climate zones (see Figure 4-3). The survey results are in red and the model results are in green. The number of samples $(\mathrm{N})$ in each category is provided in the plot label above each graph. For categories with 21 or more samples, the PDFs are continuous and calculated from histograms by using a fixed bin size of $20 \mathrm{kBtu} / \mathrm{ft}^{2} \cdot \mathrm{yr}\left(227 \mathrm{MJ} / \mathrm{m}^{2} \cdot \mathrm{yr}\right)$. When a category has 20 or fewer samples, the PDFs are discrete and each sample building is plotted individually. Some categories have no samples $(\mathrm{N}=0)$ and indicate that the CBECS sample did not cover them.

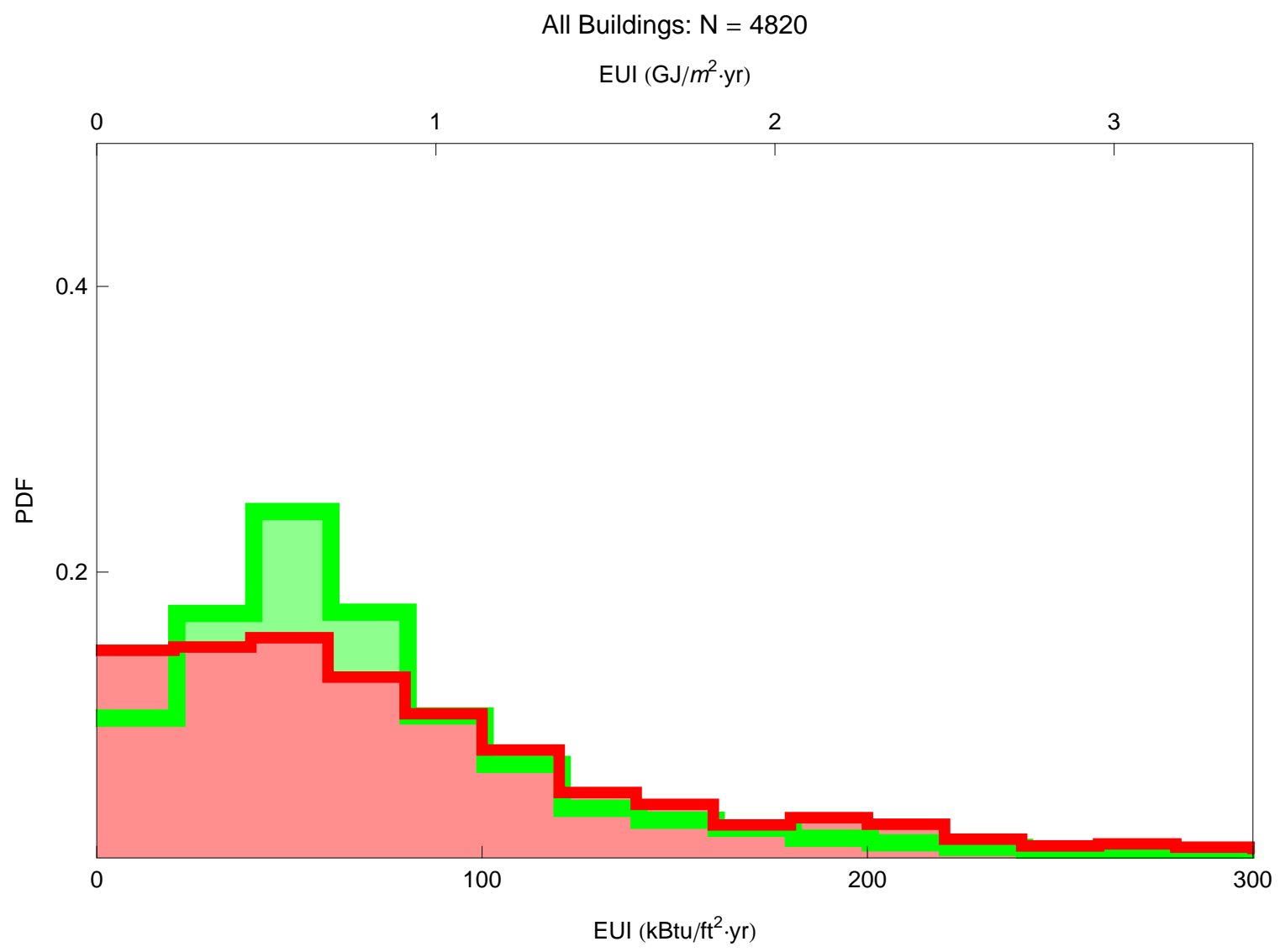

Figure A-1 PDFs for All Buildings: Survey Results in Red, Model Results in Green 
All Climates: $\mathrm{N}=4820$

EUI $\left(G J / m^{2} \cdot y r\right)$

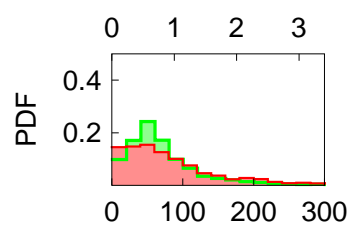

EUI $\left(\mathrm{kBtu} / \mathrm{ft}^{2} \cdot \mathrm{yr}\right)$

Climate 2B: $N=24$

EUI $\left(\mathrm{GJ} / \mathrm{m}^{2} \cdot \mathrm{yr}\right)$

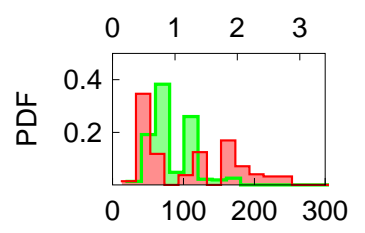

EUI (kBtu/ft $\left.{ }^{2} \cdot y r\right)$

Climate 3C: $\mathrm{N}=52$ EUI $\left(\mathrm{GJ} / m^{2} \cdot \mathrm{yr}\right)$

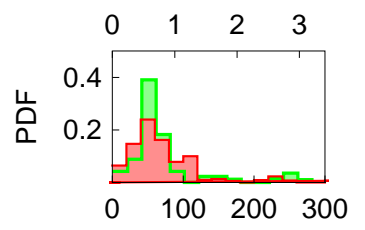

EUI (kBtu/ft $\left.{ }^{2} \cdot y r\right)$

Climate 4C: $N=50$

EUI $\left(G J / m^{2} \cdot y r\right)$

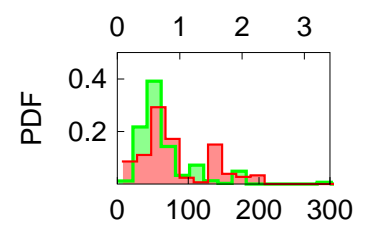

EUI $\left(\mathrm{kBtu} / \mathrm{ft}^{2} \cdot \mathrm{yr}\right)$

Climate 6A: $\mathrm{N}=519$

$$
\text { EUI }\left(\mathrm{GJ} / \mathrm{m}^{2} \cdot \mathrm{yr}\right)
$$

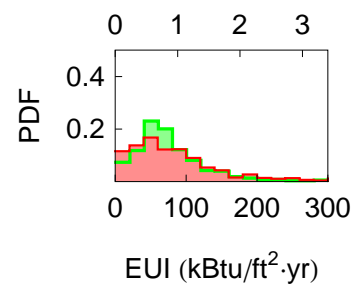

Climate 1A: $N=40$

EUI $\left(\mathrm{GJ} / \mathrm{m}^{2} \cdot \mathrm{yr}\right)$

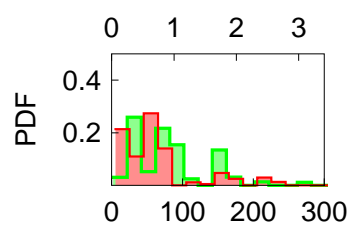

EUI $\left(\mathrm{kBtu} / \mathrm{ft}^{2} \cdot \mathrm{yr}\right)$

Climate 3A: $\mathrm{N}=464$

EUI $\left(G J / m^{2} \cdot y r\right)$

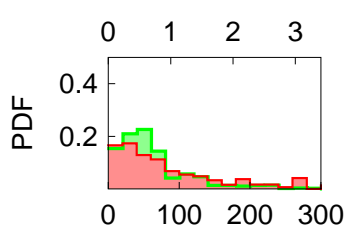

EUI $\left(k B t u / \mathrm{ft}^{2} \cdot \mathrm{yr}\right)$

Climate 4A: $\mathrm{N}=982$

EUI $\left(G J / m^{2} \cdot y r\right)$

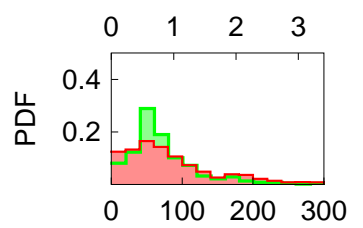

EUI $\left(k B t u / \mathrm{ft}^{2} \cdot \mathrm{yr}\right)$

Climate 5A: $N=1152$

EUI $\left(\mathrm{GJ} / \mathrm{m}^{2} \cdot \mathrm{yr}\right)$

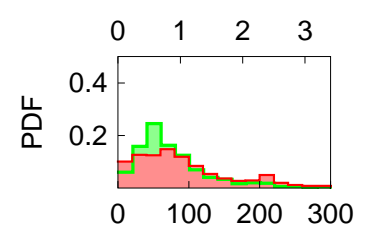

EUI $\left(\mathrm{kBtu} / \mathrm{ft}^{2} \cdot \mathrm{yr}\right)$

Climate 6B: $N=120$

EUI $\left(\mathrm{GJ} / \mathrm{m}^{2} \cdot \mathrm{yr}\right)$

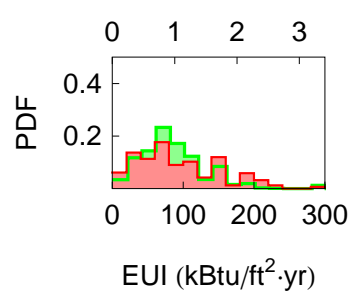

Climate 2A: $N=542$

EUI $\left(\mathrm{GJ} / \mathrm{m}^{2} \cdot \mathrm{yr}\right)$

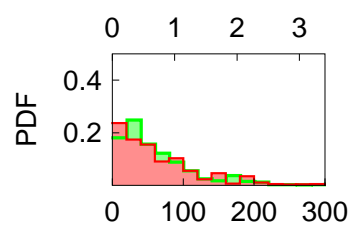

EUI (kBtu/ft $\left.{ }^{2} \cdot \mathrm{yr}\right)$

Climate 3B: $N=452$

EUI $\left(G J / m^{2} \cdot y r\right)$

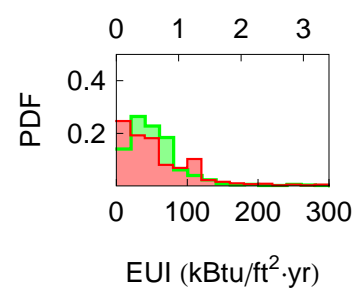

Climate 4B: $N=64$

EUI $\left(\mathrm{GJ} / m^{2} \cdot \mathrm{yr}\right)$

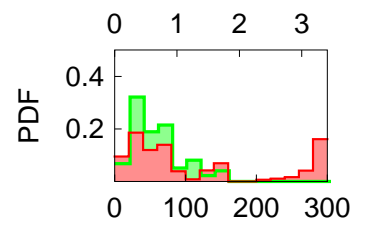

EUI $\left(k B t u / \mathrm{ft}^{2} \cdot \mathrm{yr}\right)$

Climate 5B: $\mathrm{N}=262$

EUI $\left(\mathrm{GJ} / \mathrm{m}^{2} \cdot \mathrm{yr}\right)$

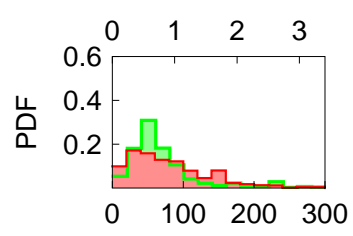

EUI (kBtu/ft $\left.{ }^{2} \cdot y r\right)$

Climate 7: $\mathrm{N}=97$

EUI $\left(\mathrm{GJ} / \mathrm{m}^{2} \cdot \mathrm{yr}\right)$

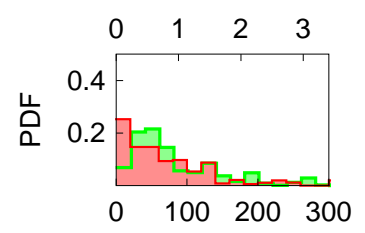

EUI $\left(\mathrm{kBtu} / \mathrm{ft}^{2} \cdot \mathrm{yr}\right)$

Figure A-2 PDFs for All Buildings in Various Climates:

Survey Results in Red, Model Results in Green 
All Climates: $\mathrm{N}=976$

EUI $\left(\mathrm{GJ} / \mathrm{m}^{2} \cdot \mathrm{yr}\right)$

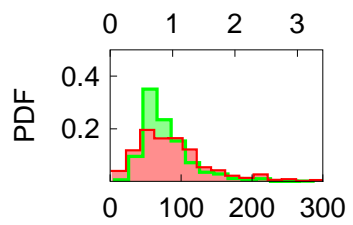

EUI $\left(\mathrm{kBtu} / \mathrm{ft}^{2} \cdot \mathrm{yr}\right)$

Climate 2B: $\mathrm{N}=7$

EUI $\left(\mathrm{GJ} / \mathrm{m}^{2} \cdot \mathrm{yr}\right)$

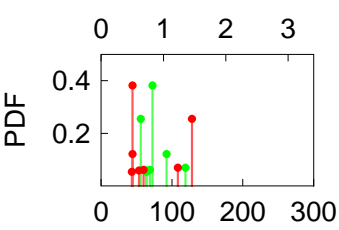

EUI (kBtu/ft $\left.{ }^{2} \cdot y r\right)$

Climate 3C: $\mathrm{N}=18$

EUI $\left(\mathrm{GJ} / m^{2} \cdot \mathrm{yr}\right)$

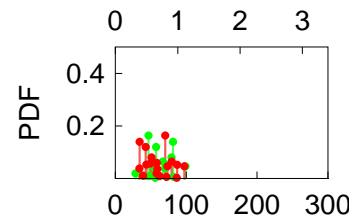

EUI (kBtu/ft $\left.{ }^{2} \cdot y r\right)$

Climate 4C: $\mathrm{N}=21$

EUI $\left(\mathrm{GJ} / m^{2} \cdot \mathrm{yr}\right)$

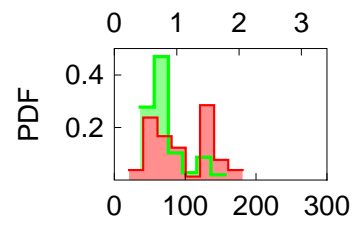

EUI $\left(\mathrm{kBtu} / \mathrm{ft}^{2} \cdot \mathrm{yr}\right)$

Climate $6 \mathrm{~A}: \mathrm{N}=93$

EUI $\left(\mathrm{GJ} / \mathrm{m}^{2} \cdot \mathrm{yr}\right)$

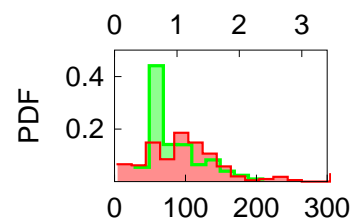

EUI $\left(k B t u / \mathrm{ft}^{2} \cdot y r\right)$
Climate 1A: $N=6$

EUI (GJ $\left./ m^{2} \cdot y r\right)$

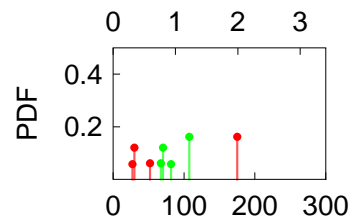

EUI $\left(k B t u / \mathrm{ft}^{2} \cdot \mathrm{yr}\right)$

Climate 3A: $N=73$

EUI $\left(\mathrm{GJ} / \mathrm{m}^{2} \cdot \mathrm{yr}\right)$

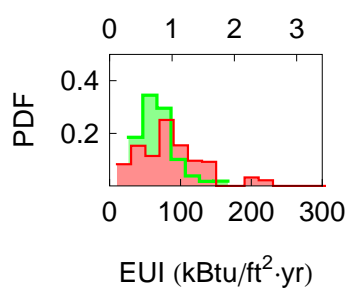

Climate 4A: $N=211$

EUI $\left(\mathrm{GJ} / m^{2} \cdot \mathrm{yr}\right)$

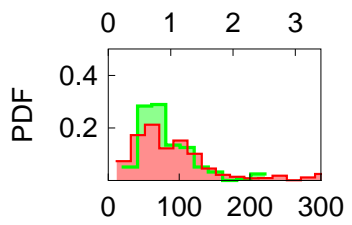

EUI $\left(k B t u / \mathrm{ft}^{2} \cdot \mathrm{yr}\right)$

Climate 5A: $N=253$

$\mathrm{EUI}\left(\mathrm{GJ} / m^{2} \cdot \mathrm{yr}\right)$

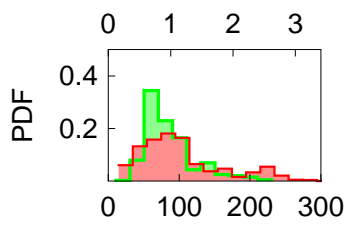

EUI (kBtu/ft $\left.{ }^{2} \cdot y r\right)$

Climate 6B: $\mathrm{N}=26$

EUI $\left(\mathrm{GJ} / \mathrm{m}^{2} \cdot \mathrm{yr}\right)$

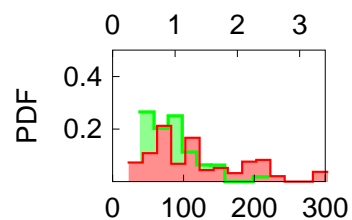

EUI $\left(\mathrm{kBtu} / \mathrm{ft}^{2} \cdot \mathrm{yr}\right)$
Climate 2A: $\mathrm{N}=99$

EUI $\left(\mathrm{GJ} / \mathrm{m}^{2} \cdot \mathrm{yr}\right)$

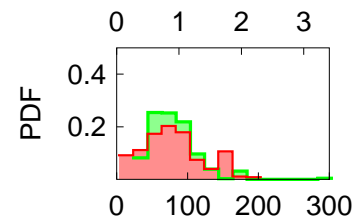

EUI $\left(k B t u / \mathrm{ft}^{2} \cdot \mathrm{yr}\right)$

Climate 3B: $N=108$

EUI $\left(\mathrm{GJ} / \mathrm{m}^{2} \cdot \mathrm{yr}\right)$

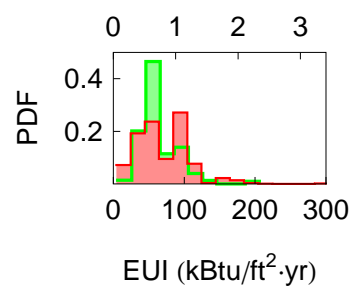

Climate 4B: $N=6$

EUI (GJ $\left./ m^{2} \cdot y r\right)$

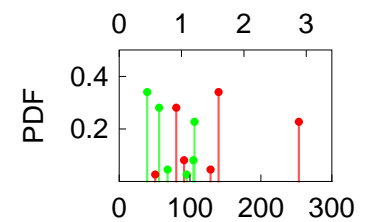

EUI $\left(\mathrm{kBtu} / \mathrm{ft}^{2} \cdot \mathrm{yr}\right)$

Climate 5B: $N=33$

EUI $\left(\mathrm{GJ} / m^{2} \cdot \mathrm{yr}\right)$

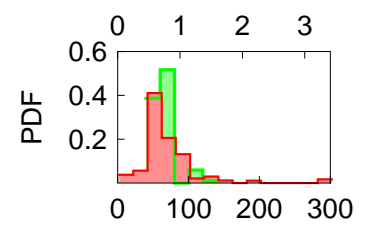

EUI (kBtu/ft $\left.{ }^{2} \cdot y r\right)$

Climate $7: \mathrm{N}=22$

EUI $\left(\mathrm{GJ} / \mathrm{m}^{2} \cdot \mathrm{yr}\right)$

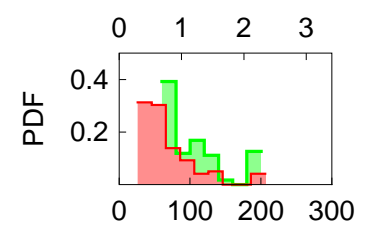

EUI $\left(k B t u / f t^{2} \cdot y r\right)$

Figure A-3 PDFs for Office Buildings in Various Climates:

Survey Results in Red, Model Results in Green 
All Climates: $N=473$

EUI $\left(\mathrm{GJ} / \mathrm{m}^{2} \cdot \mathrm{yr}\right)$

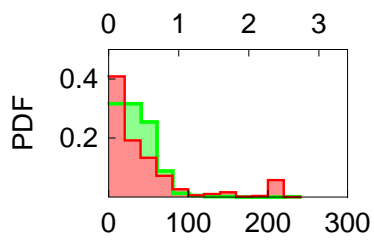

EUI $\left(k B t u / \mathrm{ft}^{2} \cdot \mathrm{yr}\right)$

Climate 2B: $\mathrm{N}=0$

Climate 3C: $\mathrm{N}=2$

EUI $\left(\mathrm{GJ} / \mathrm{m}^{2} \cdot \mathrm{yr}\right)$

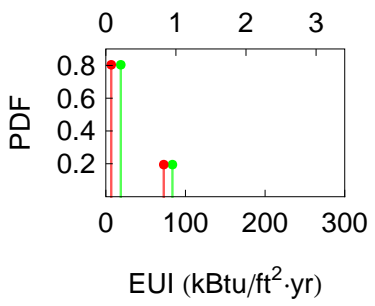

Climate 4C: $\mathrm{N}=6$

EUI $\left(\mathrm{GJ} / m^{2} \cdot \mathrm{yr}\right)$

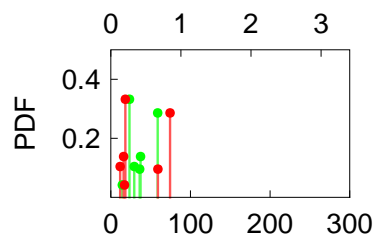

EUI $\left(k B t u / \mathrm{ft}^{2} \cdot \mathrm{yr}\right)$

Climate 6A: $N=50$

EUI $\left(\mathrm{GJ} / m^{2} \cdot \mathrm{yr}\right)$

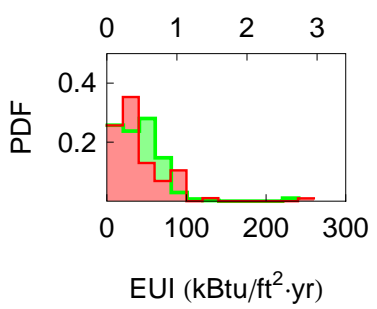

Climate 1A: $\mathrm{N}=5$

EUI $\left(\mathrm{GJ} / \mathrm{m}^{2} \cdot \mathrm{yr}\right)$

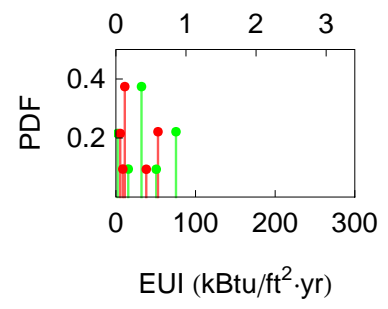

Climate 3A: $N=49$

EUI $\left(\mathrm{GJ} / \mathrm{m}^{2} \cdot \mathrm{yr}\right)$

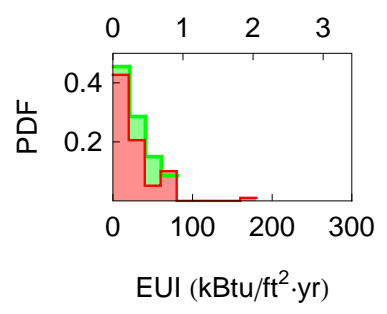

Climate 4A: $N=93$

EUI (GJ $\left./ m^{2} \cdot y r\right)$

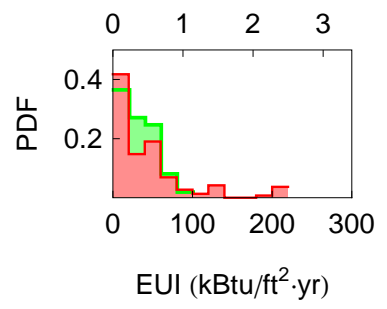

Climate 5A: $\mathrm{N}=94$

EUI $\left(\mathrm{GJ} / \mathrm{m}^{2} \cdot \mathrm{yr}\right)$

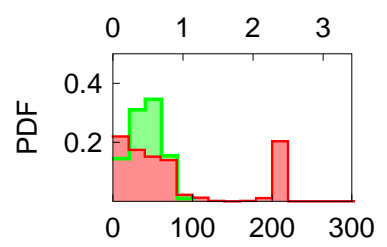

EUI (kBtu/ft $\left.{ }^{2} \cdot \mathrm{yr}\right)$

Climate 6B: $\mathrm{N}=10$

EUI (GJ $\left./ m^{2} \cdot y r\right)$

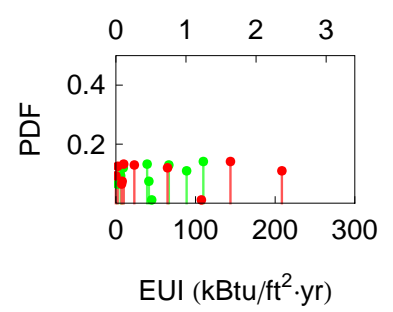

Climate 2A: $\mathrm{N}=77$

EUI $\left(\mathrm{GJ} / m^{2} \cdot \mathrm{yr}\right)$

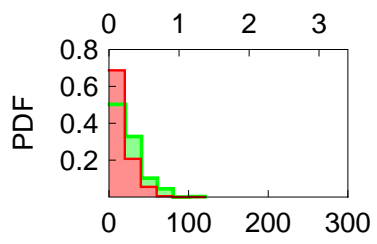

EUI $\left(\mathrm{kBtu} / \mathrm{ft}^{2} \cdot \mathrm{yr}\right)$

Climate 3B: $\mathrm{N}=50$

EUI $\left(\mathrm{GJ} / \mathrm{m}^{2} \cdot \mathrm{yr}\right)$

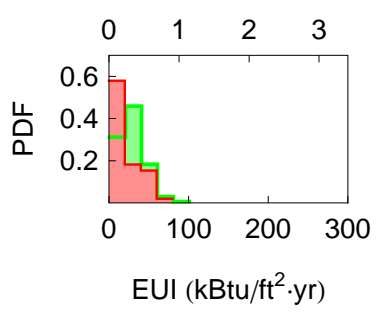

Climate 4B: $N=6$

EUI $\left(\mathrm{GJ} / \mathrm{m}^{2} \cdot \mathrm{yr}\right)$

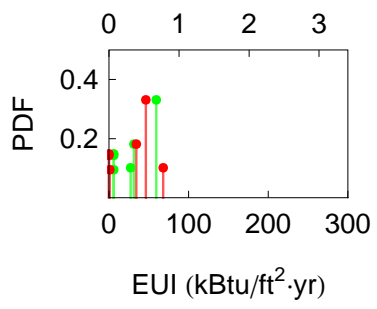

Climate 5B: $\mathrm{N}=21$

EUI $\left(\mathrm{GJ} / \mathrm{m}^{2} \cdot \mathrm{yr}\right)$

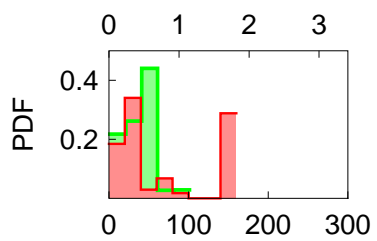

EUI $\left(\mathrm{kBtu} / \mathrm{ft}^{2} \cdot \mathrm{yr}\right)$

Climate 7: $N=10$

EUI $\left(\mathrm{GJ} / \mathrm{m}^{2} \cdot \mathrm{yr}\right)$

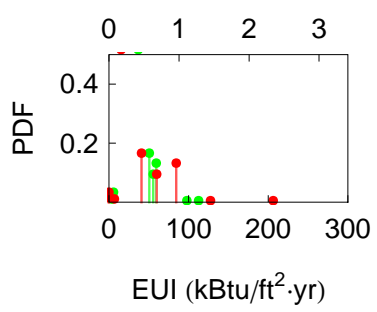

Figure A-4 PDFs for Nonrefrigerated Warehouse Buildings in Various Climates:

Survey Results in Red, Model Results in Green 
All Climates: $\mathrm{N}=649$

EUI $\left(\mathrm{GJ} / \mathrm{m}^{2} \cdot \mathrm{yr}\right)$

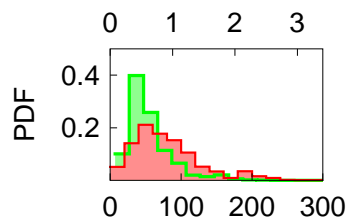

EUI $\left(\mathrm{kBtu} / \mathrm{ft}^{2} \cdot \mathrm{yr}\right)$

Climate 2B: $\mathrm{N}=5$

EUI $\left(\mathrm{GJ} / \mathrm{m}^{2} \cdot \mathrm{yr}\right)$

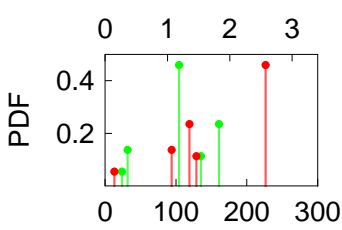

EUI (kBtu/ft $\left.{ }^{2} \cdot y r\right)$

Climate 3C: $\mathrm{N}=8$

EUI $\left(\mathrm{GJ} / m^{2} \cdot \mathrm{yr}\right)$

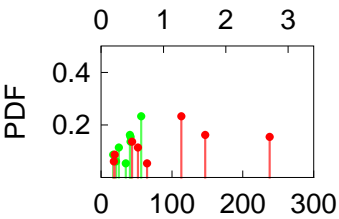

EUI (kBtu/ft $\left.{ }^{2} \cdot y r\right)$

Climate 4C: $\mathrm{N}=8$

EUI $\left(\mathrm{GJ} / m^{2} \cdot \mathrm{yr}\right)$

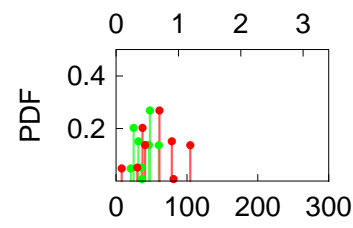

EUI (kBtu/ft $\left.{ }^{2} \cdot y r\right)$

Climate 6A: $N=61$

EUI $\left(\mathrm{GJ} / \mathrm{m}^{2} \cdot \mathrm{yr}\right)$

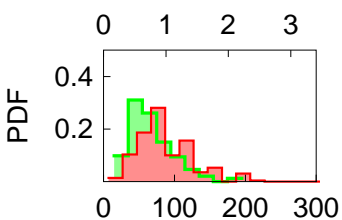

EUI $\left(k B t u / \mathrm{ft}^{2} \cdot \mathrm{yr}\right)$
Climate 1A: $\mathrm{N}=4$

EUI $\left(\mathrm{GJ} / \mathrm{m}^{2} \cdot \mathrm{yr}\right)$

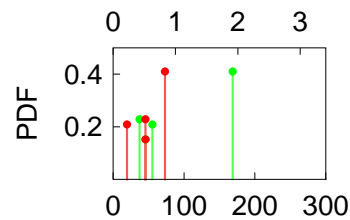

EUI $\left(\mathrm{kBtu} / \mathrm{ft}^{2} \cdot \mathrm{yr}\right)$

Climate 3A: $N=62$

EUI $\left(\mathrm{GJ} / \mathrm{m}^{2} \cdot \mathrm{yr}\right)$

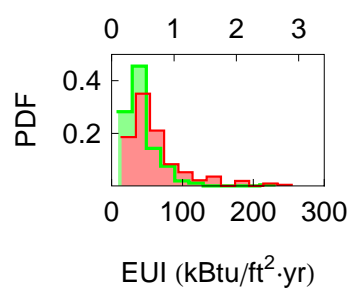

Climate 4A: $\mathrm{N}=124$

EUI $\left(\mathrm{GJ} / m^{2} \cdot \mathrm{yr}\right)$

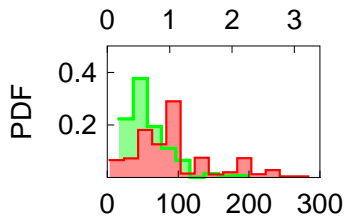

EUI (kBtu/ft $\left.{ }^{2} \cdot y r\right)$

Climate 5A: $\mathrm{N}=139$

EUI $\left(\mathrm{GJ} / m^{2} \cdot \mathrm{yr}\right)$

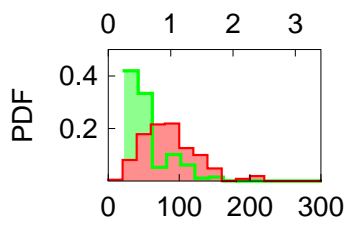

EUI $\left(\mathrm{kBtu} / \mathrm{ft}^{2} \cdot \mathrm{yr}\right)$

Climate 6B: $\mathrm{N}=17$

EUI $\left(\mathrm{GJ} / \mathrm{m}^{2} \cdot \mathrm{yr}\right)$

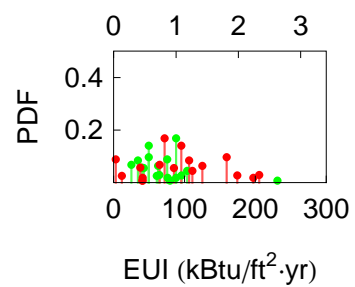

Climate 2A: $N=91$

EUI $\left(\mathrm{GJ} / \mathrm{m}^{2} \cdot \mathrm{yr}\right)$

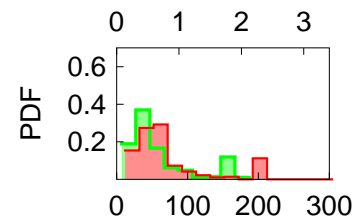

EUI $\left(k B t u / f^{2} \cdot y r\right)$

Climate 3B: $N=59$

EUI $\left(\mathrm{GJ} / \mathrm{m}^{2} \cdot \mathrm{yr}\right)$

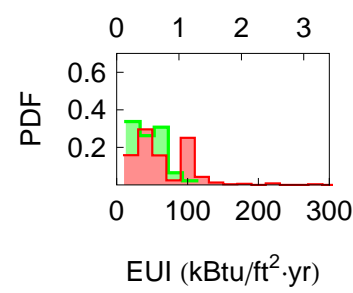

Climate 4B: $\mathrm{N}=9$

EUI $\left(\mathrm{GJ} / m^{2} \cdot \mathrm{yr}\right)$

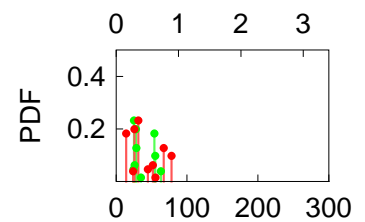

EUI $\left(\mathrm{kBtu} / \mathrm{ft}^{2} \cdot \mathrm{yr}\right)$

Climate 5B: $N=58$

EUI $\left(\mathrm{GJ} / m^{2} \cdot \mathrm{yr}\right)$

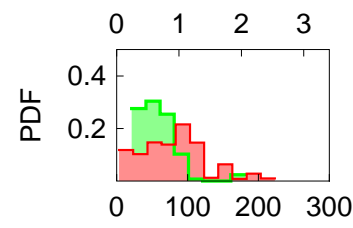

EUI (kBtu/ft $\left.{ }^{2} \cdot y r\right)$

Climate 7: $\mathrm{N}=4$

EUI $\left(\mathrm{GJ} / m^{2} \cdot \mathrm{yr}\right)$

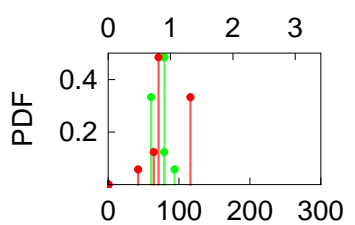

EUI $\left(\mathrm{kBtu} / \mathrm{ft}^{2} \cdot \mathrm{yr}\right)$

Figure A-5 PDFs for Education Buildings in Various Climates:

Survey Results in Red, Model Results in Green 
All Climates: $\mathrm{N}=355$

EUI $\left(\mathrm{GJ} / \mathrm{m}^{2} \cdot \mathrm{yr}\right)$

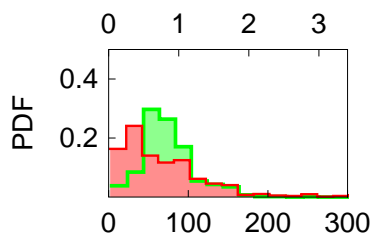

EUI $\left(k B t u / \mathrm{ft}^{2} \cdot \mathrm{yr}\right)$

Climate 2B: $\mathrm{N}=2$

EUI $\left(\mathrm{GJ} / \mathrm{m}^{2} \cdot \mathrm{yr}\right)$

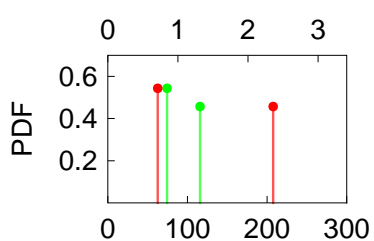

EUI $\left(k B t u / \mathrm{ft}^{2} \cdot \mathrm{yr}\right)$

Climate 3C: $\mathrm{N}=4$

EUI $\left(\mathrm{GJ} / m^{2} \cdot \mathrm{yr}\right)$

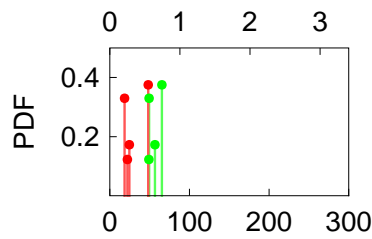

EUI (kBtu/ft $\left.{ }^{2} \cdot y r\right)$

Climate 4C: $N=0$

Climate 6A: $\mathrm{N}=27$

EUI $\left(\mathrm{GJ} / m^{2} \cdot \mathrm{yr}\right)$

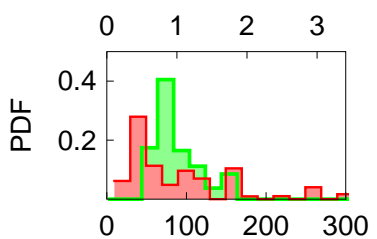

EUI $\left(k B t u / \mathrm{ft}^{2} \cdot \mathrm{yr}\right)$
Climate 1A: $\mathrm{N}=4$

EUI (GJ $\left./ m^{2} \cdot y r\right)$

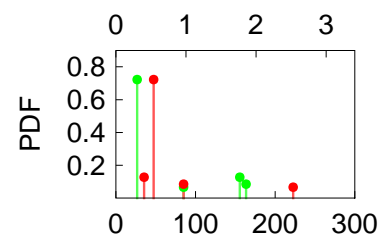

EUI (kBtu/ft $\left.{ }^{2} \cdot \mathrm{yr}\right)$

Climate 3A: $\mathrm{N}=52$

EUI $\left(\mathrm{GJ} / \mathrm{m}^{2} \cdot \mathrm{yr}\right)$

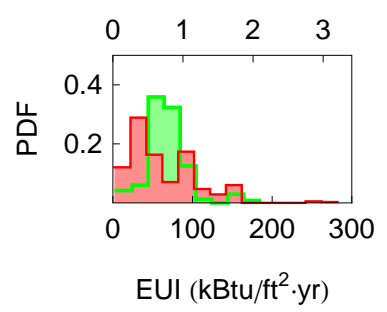

Climate 4A: $N=80$

EUI $\left(\mathrm{GJ} / \mathrm{m}^{2} \cdot \mathrm{yr}\right)$

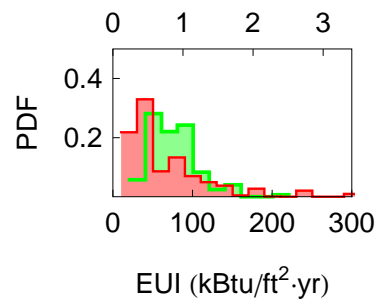

Climate 5A: $N=73$

EUI $\left(\mathrm{GJ} / \mathrm{m}^{2} \cdot \mathrm{yr}\right)$

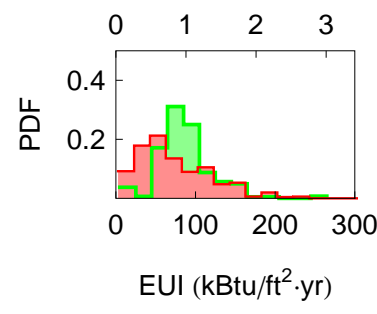

Climate 6B: $\mathrm{N}=7$

EUI $\left(\mathrm{GJ} / \mathrm{m}^{2} \cdot \mathrm{yr}\right)$

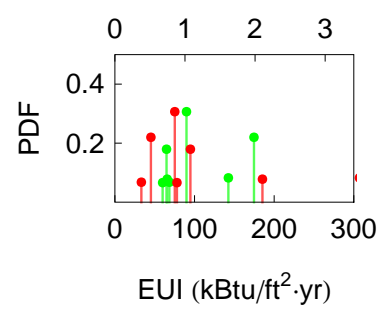

Climate 2A: $\mathrm{N}=34$

EUI $\left(\mathrm{GJ} / m^{2} \cdot \mathrm{yr}\right)$

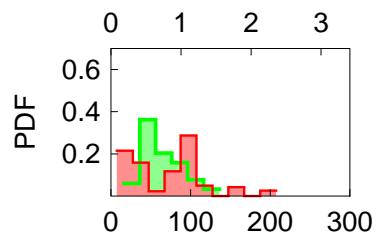

EUI $\left(\mathrm{kBtu} / \mathrm{ft}^{2} \cdot \mathrm{yr}\right)$

Climate 3B: $\mathrm{N}=36$

EUI $\left(\mathrm{GJ} / \mathrm{m}^{2} \cdot \mathrm{yr}\right)$

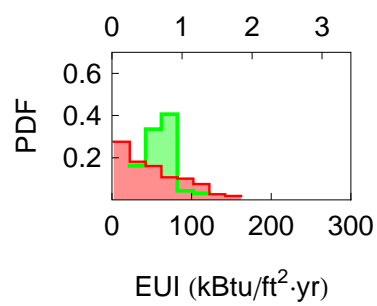

Climate 4B: $N=6$

EUI $\left(\mathrm{GJ} / \mathrm{m}^{2} \cdot \mathrm{yr}\right)$

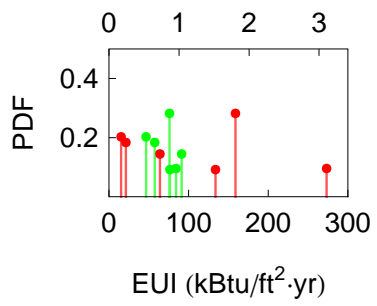

Climate 5B: $\mathrm{N}=23$

EUI $\left(\mathrm{GJ} / m^{2} \cdot \mathrm{yr}\right)$

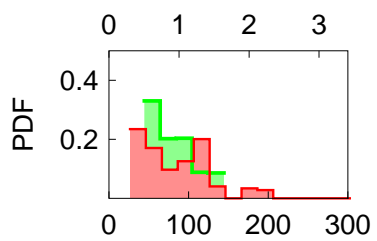

EUI $\left(\mathrm{kBtu} / \mathrm{ft}^{2} \cdot \mathrm{yr}\right)$

Climate 7: $\mathrm{N}=7$

EUI $\left(\mathrm{GJ} / \mathrm{m}^{2} \cdot \mathrm{yr}\right)$

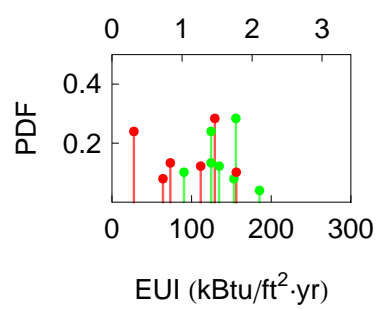

Figure A-6 PDFs for Retail (except Malls) Buildings in Various Climates:

Survey Results in Red, Model Results in Green 
All Climates: $\mathrm{N}=279$

EUI $\left(\mathrm{GJ} / \mathrm{m}^{2} \cdot \mathrm{yr}\right)$

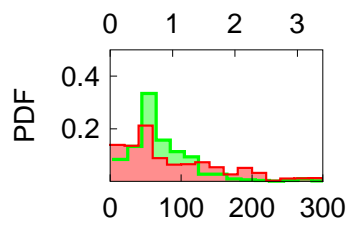

EUI $\left(\mathrm{kBtu} / \mathrm{ft}^{2} \cdot \mathrm{yr}\right)$

Climate 2B: $\mathrm{N}=2$

EUI $\left(\mathrm{GJ} / \mathrm{m}^{2} \cdot \mathrm{yr}\right)$

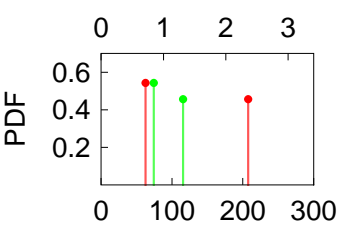

EUI (kBtu/ft $\left.{ }^{2} \cdot y r\right)$

Climate 3C: $\mathrm{N}=3$

EUI $\left(\mathrm{GJ} / m^{2} \cdot \mathrm{yr}\right)$

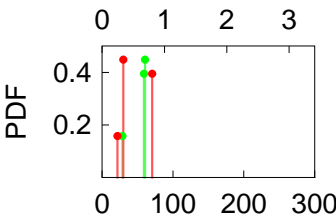

EUI (kBtu/ft $\left.{ }^{2} \cdot y r\right)$

Climate 4C: $\mathrm{N}=5$

EUI $\left(\mathrm{GJ} / m^{2} \cdot \mathrm{yr}\right)$

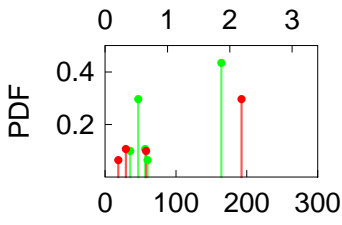

EUI $\left(\mathrm{kBtu} / \mathrm{ft}^{2} \cdot \mathrm{yr}\right)$

Climate 6A: $\mathrm{N}=37$

EUI $\left(\mathrm{GJ} / m^{2} \cdot \mathrm{yr}\right)$

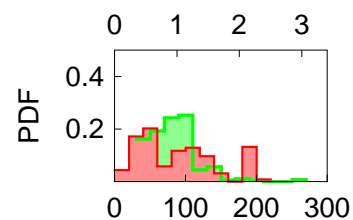

EUI $\left(k B t u / \mathrm{ft}^{2} \cdot \mathrm{yr}\right)$
Climate 1A: $\mathrm{N}=3$

EUI $\left(\mathrm{GJ} / \mathrm{m}^{2} \cdot \mathrm{yr}\right)$

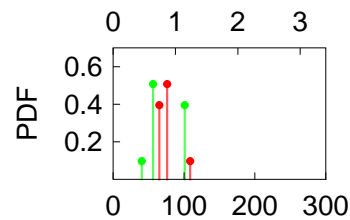

EUI $\left(\mathrm{kBtu} / \mathrm{ft}^{2} \cdot \mathrm{yr}\right)$

Climate 3A: $\mathrm{N}=25$

EUI $\left(\mathrm{GJ} / \mathrm{m}^{2} \cdot \mathrm{yr}\right)$

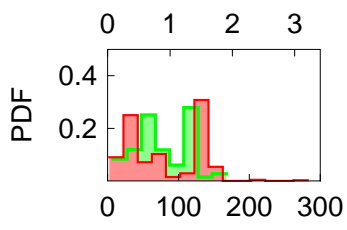

EUI $\left(k B t u / \mathrm{ft}^{2} \cdot \mathrm{yr}\right)$

Climate 4A: $N=47$

EUI $\left(\mathrm{GJ} / \mathrm{m}^{2} \cdot \mathrm{yr}\right)$

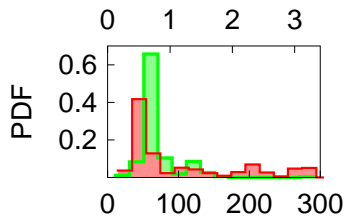

EUI $\left(\mathrm{kBtu} / \mathrm{ft}^{2} \cdot \mathrm{yr}\right)$

Climate 5A: $N=62$

EUI $\left(\mathrm{GJ} / m^{2} \cdot \mathrm{yr}\right)$

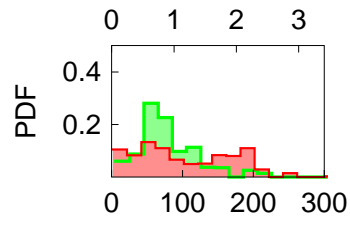

EUI $\left(\mathrm{kBtu} / \mathrm{ft}^{2} \cdot \mathrm{yr}\right)$

Climate 6B: $\mathrm{N}=6$

EUI $\left(\mathrm{GJ} / \mathrm{m}^{2} \cdot \mathrm{yr}\right)$

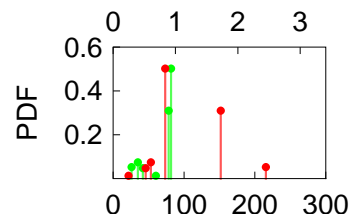

EUI $\left(k B t u / \mathrm{ft}^{2} \cdot \mathrm{yr}\right)$
Climate 2A: $\mathrm{N}=36$

EUI $\left(\mathrm{GJ} / m^{2} \cdot \mathrm{yr}\right)$

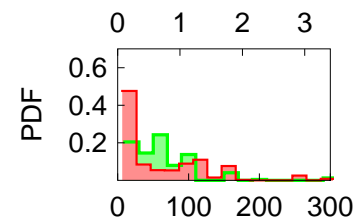

EUI $\left(\mathrm{kBtu} / \mathrm{ft}^{2} \cdot \mathrm{yr}\right)$

Climate 3B: $N=26$

EUI $\left(\mathrm{GJ} / \mathrm{m}^{2} \cdot \mathrm{yr}\right)$

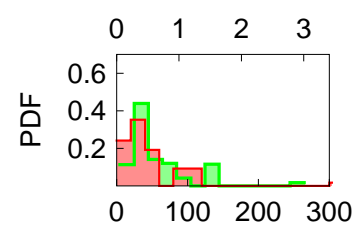

EUI (kBtu/ft $\left.{ }^{2} \cdot y r\right)$

Climate 4B: $N=5$

EUI (GJ $\left./ m^{2} \cdot y r\right)$

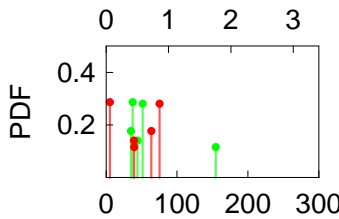

EUI $\left(k B t u / \mathrm{ft}^{2} \cdot \mathrm{yr}\right)$

Climate 5B: $N=14$

EUI $\left(\mathrm{GJ} / m^{2} \cdot \mathrm{yr}\right)$

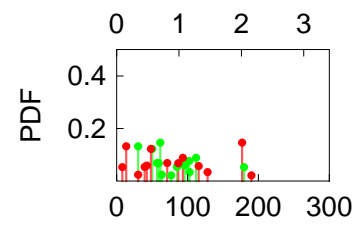

EUI $\left(k B t u / \mathrm{ft}^{2} \cdot \mathrm{yr}\right)$

Climate 7: $\mathrm{N}=9$

EUI $\left(\mathrm{GJ} / m^{2} \cdot \mathrm{yr}\right)$

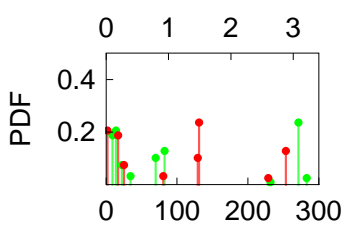

EUI $\left(k B t u / f t^{2} \cdot y r\right)$

Figure A-7 PDFs for Public Assembly Buildings in Various Climates: Survey results in red, Model results in Green 
All Climates: $\mathrm{N}=370$

EUI $\left(\mathrm{GJ} / m^{2} \cdot \mathrm{yr}\right)$

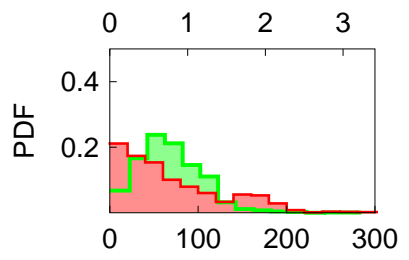

EUI (kBtu/ft $\left.{ }^{2} \cdot y r\right)$

Climate 2B: $\mathrm{N}=0$

Climate 3C: $\mathrm{N}=2$

EUI $\left(\mathrm{GJ} / \mathrm{m}^{2} \cdot \mathrm{yr}\right)$

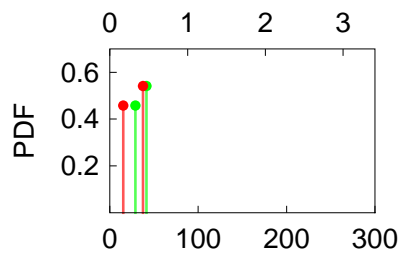

$\mathrm{EUI}\left(\mathrm{kBtu} / \mathrm{ft}^{2} \cdot \mathrm{yr}\right)$

Climate 4C: $\mathrm{N}=0$

Climate 6A: $\mathrm{N}=54$

EUI $\left(\mathrm{GJ} / \mathrm{m}^{2} \cdot \mathrm{yr}\right)$

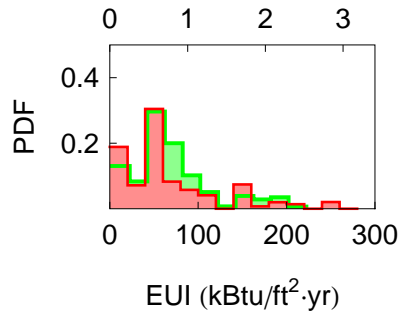

Climate 1A: $\mathrm{N}=2$

EUI $\left(\mathrm{GJ} / m^{2} \cdot \mathrm{yr}\right)$

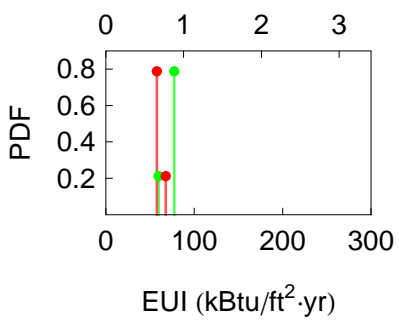

Climate 3A: $N=43$

EUI $\left(\mathrm{GJ} / \mathrm{m}^{2} \cdot \mathrm{yr}\right)$

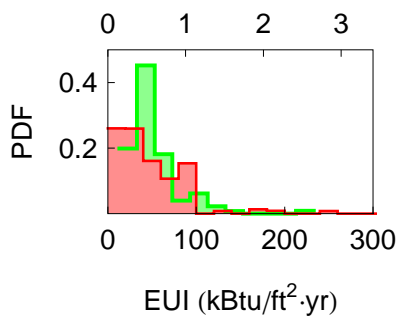

Climate 4A: $\mathrm{N}=76$

EUI $\left(\mathrm{GJ} / \mathrm{m}^{2} \cdot \mathrm{yr}\right)$

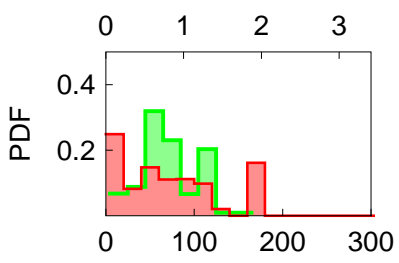

EUI $\left(\mathrm{kBtu} / \mathrm{ft}^{2} \cdot \mathrm{yr}\right)$

Climate 5A: $N=100$

EUI $\left(\mathrm{GJ} / \mathrm{m}^{2} \cdot \mathrm{yr}\right)$

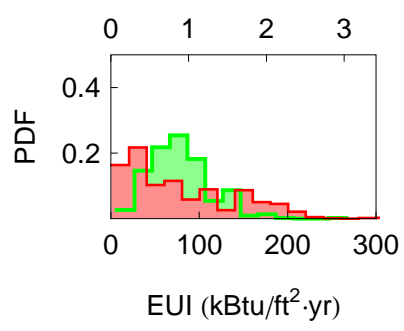

Climate 6B: $\mathrm{N}=13$

EUI $\left(\mathrm{GJ} / \mathrm{m}^{2} \cdot \mathrm{yr}\right)$

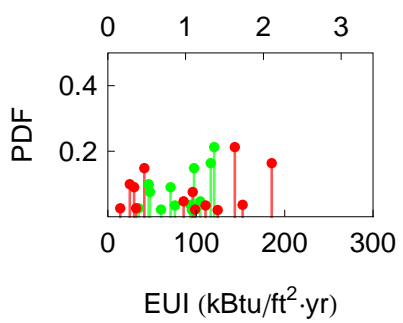

Climate 2A: $\mathrm{N}=20$

EUI $\left(\mathrm{GJ} / m^{2} \cdot \mathrm{yr}\right)$

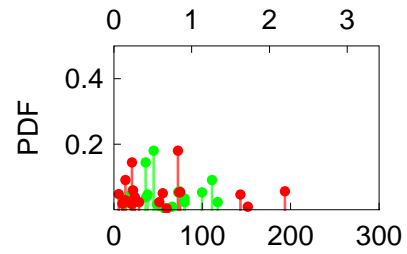

EUI $\left(\mathrm{kBtu} / \mathrm{ft}^{2} \cdot \mathrm{yr}\right)$

Climate 3B: $N=21$

EUI $\left(\mathrm{GJ} / m^{2} \cdot \mathrm{yr}\right)$

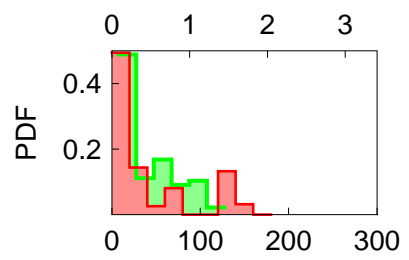

EUI $\left(\mathrm{kBtu} / \mathrm{ft}^{2} \cdot \mathrm{yr}\right)$

Climate 4B: $\mathrm{N}=5$

EUI $\left(\mathrm{GJ} / m^{2} \cdot \mathrm{yr}\right)$

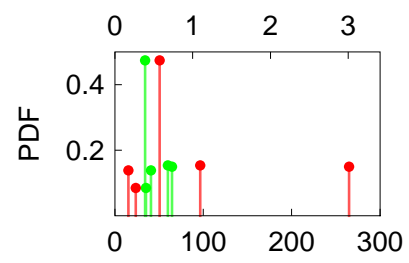

EUI $\left(\mathrm{kBtu} / \mathrm{ft}^{2} \cdot \mathrm{yr}\right)$

Climate 5B: $\mathrm{N}=20$

EUI $\left(\mathrm{GJ} / \mathrm{m}^{2} \cdot \mathrm{yr}\right)$

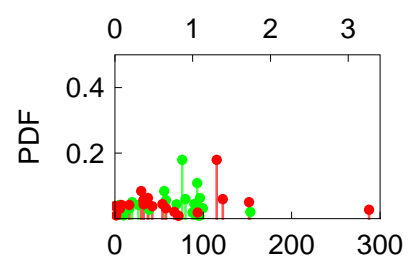

EUI $\left(\mathrm{kBtu} / \mathrm{ft}^{2} \cdot \mathrm{yr}\right)$

Climate 7: $N=13$

EUI $\left(\mathrm{GJ} / \mathrm{m}^{2} \cdot \mathrm{yr}\right)$

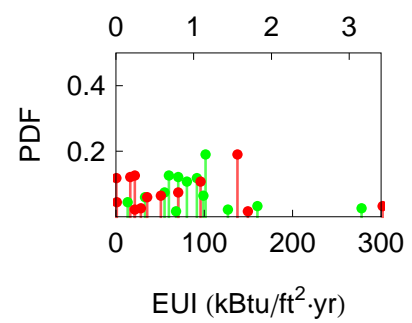

Figure A-8 PDFs for Service Buildings in Various Climates: Survey Results in Red, Model Results in Green 
All Climates: $\mathrm{N}=311$

EUI $\left(\mathrm{GJ} / \mathrm{m}^{2} \cdot \mathrm{yr}\right)$

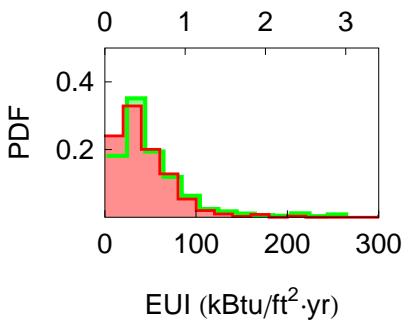

Climate 2B: $\mathrm{N}=0$

Climate 3C: $\mathrm{N}=0$

Climate 4C: $\mathrm{N}=0$

Climate 6A: $\mathrm{N}=45$

EUI $\left(\mathrm{GJ} / \mathrm{m}^{2} \cdot \mathrm{yr}\right)$

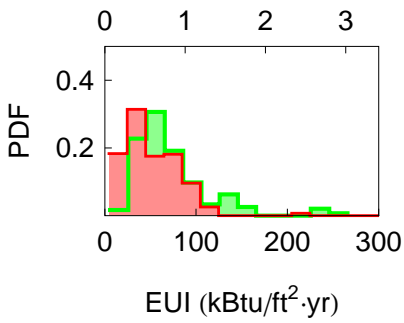

Climate $1 \mathrm{~A}: \mathrm{N}=0$

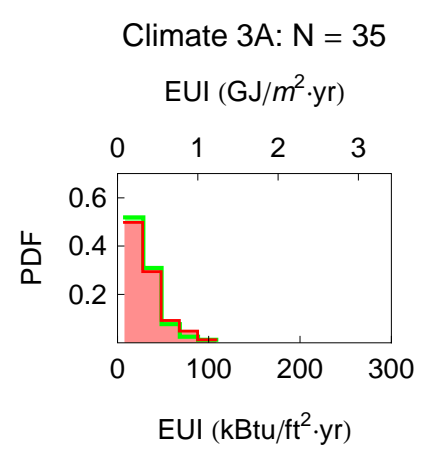

Climate 4A: $N=64$

EUI $\left(\mathrm{GJ} / \mathrm{m}^{2} \cdot \mathrm{yr}\right)$

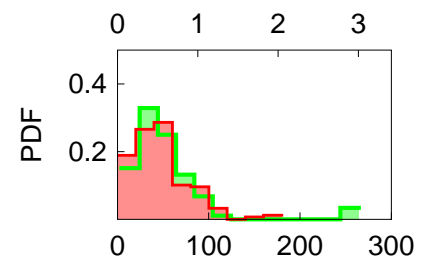

EUI $\left(k B t u / \mathrm{ft}^{2} \cdot \mathrm{yr}\right)$

Climate 5A: $\mathrm{N}=82$

EUI $\left(\mathrm{GJ} / \mathrm{m}^{2} \cdot \mathrm{yr}\right)$

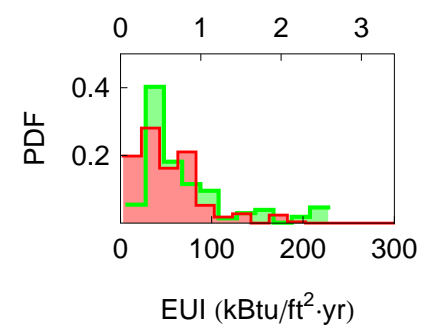

Climate 6B: $\mathrm{N}=6$

EUI (GJ $\left./ m^{2} \cdot y r\right)$

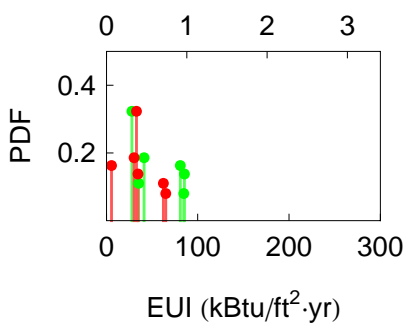

Climate 2A: $\mathrm{N}=31$

EUI $\left(\mathrm{GJ} / \mathrm{m}^{2} \cdot \mathrm{yr}\right)$

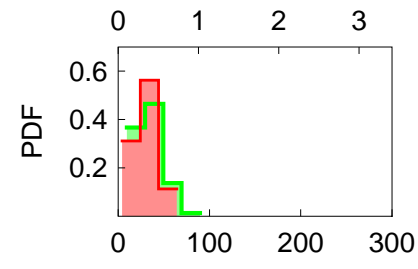

EUI $\left(\mathrm{kBtu} / \mathrm{ft}^{2} \cdot \mathrm{yr}\right)$

Climate 3B: $N=29$

EUI (GJ $\left./ m^{2} \cdot y r\right)$

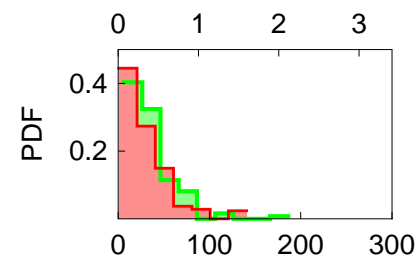

EUI $\left(\mathrm{kBtu} / \mathrm{ft}^{2} \cdot \mathrm{yr}\right)$

Climate 4B: $\mathrm{N}=6$

EUI (GJ $\left./ m^{2} \cdot y r\right)$

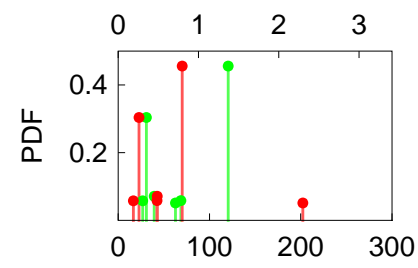

EUI $\left(\mathrm{kBtu} / \mathrm{ft}^{2} \cdot \mathrm{yr}\right)$

Climate 5B: $\mathrm{N}=11$

EUI $\left(\mathrm{GJ} / \mathrm{m}^{2} \cdot \mathrm{yr}\right)$

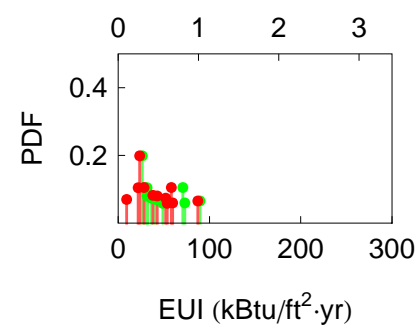

Climate 7: $\mathrm{N}=0$

Figure A-9 PDFs for Religious Worship Buildings in Various Climates:

Survey Results in Red, Model Results in Green 
All Climates: $\mathrm{N}=260$

EUI (GJ $\left./ m^{2} \cdot y r\right)$

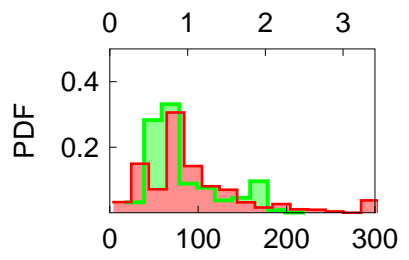

EUI $\left(\mathrm{kBtu} / \mathrm{ft}^{2} \cdot \mathrm{yr}\right)$

Climate 2B: $\mathrm{N}=0$

Climate 3C: $\mathrm{N}=0$

$$
\begin{gathered}
\text { Climate 4C: } N=3 \\
\text { EUI }\left(\mathrm{GJ} / \mathrm{m}^{2} \cdot \mathrm{yr}\right)
\end{gathered}
$$

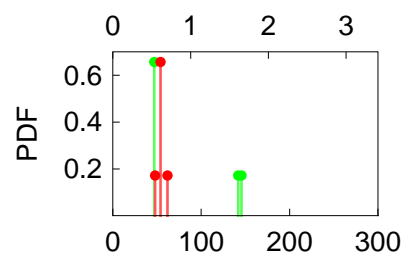

EUI (kBtu/ft $\left.{ }^{2} \cdot y r\right)$

Climate 6A: $N=26$

EUI $\left(\mathrm{GJ} / \mathrm{m}^{2} \cdot \mathrm{yr}\right)$

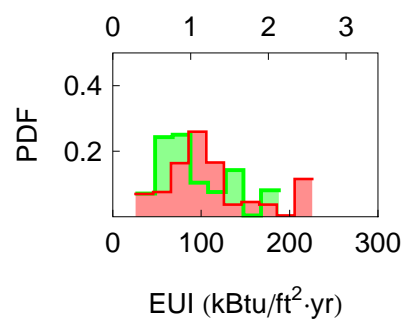

Climate 1A: $N=7$

EUI $\left(\mathrm{GJ} / m^{2} \cdot \mathrm{yr}\right)$

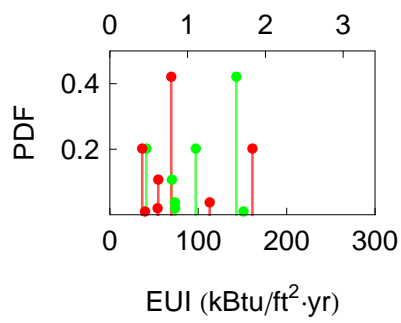

Climate 3A: $\mathrm{N}=20$

EUI $\left(\mathrm{GJ} / \mathrm{m}^{2} \cdot \mathrm{yr}\right)$

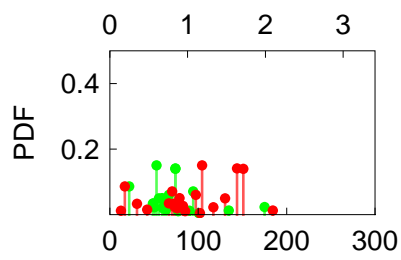

EUI $\left(k B t u / \mathrm{ft}^{2} \cdot \mathrm{yr}\right)$

Climate 4A: $\mathrm{N}=58$

EUI $\left(\mathrm{GJ} / \mathrm{m}^{2} \cdot \mathrm{yr}\right)$

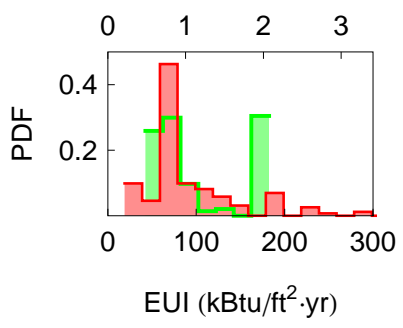

Climate 5A: $N=51$

EUI (GJ $\left./ m^{2} \cdot y r\right)$

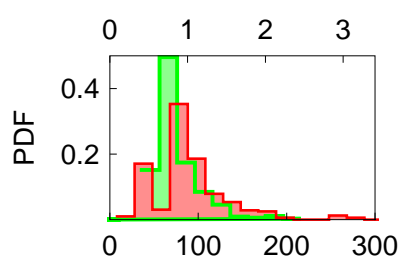

EUI $\left(\mathrm{kBtu} / \mathrm{ft}^{2} \cdot \mathrm{yr}\right)$

Climate 6B: $\mathrm{N}=8$

EUI (GJ $\left./ m^{2} \cdot y r\right)$

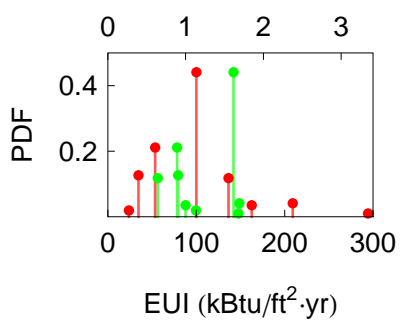

Climate 2A: $N=31$

EUI $\left(\mathrm{GJ} / \mathrm{m}^{2} \cdot \mathrm{yr}\right)$

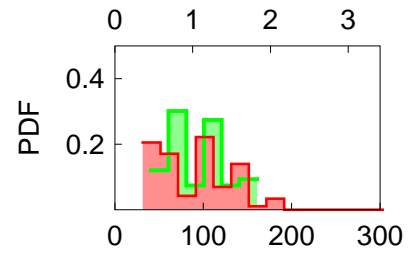

EUI $\left(k B t u / \mathrm{ft}^{2} \cdot \mathrm{yr}\right)$

Climate 3B: $\mathrm{N}=22$

EUI $\left(\mathrm{GJ} / m^{2} \cdot \mathrm{yr}\right)$

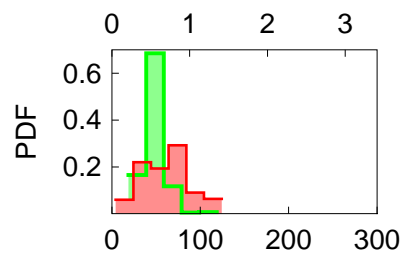

EUI $\left(\mathrm{kBtu} / \mathrm{ft}^{2} \cdot \mathrm{yr}\right)$

Climate 4B: $\mathrm{N}=7$

EUI $\left(\mathrm{GJ} / m^{2} \cdot \mathrm{yr}\right)$

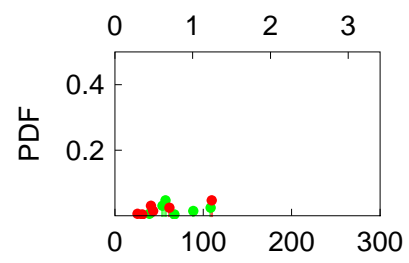

EUI $\left(\mathrm{kBtu} / \mathrm{ft}^{2} \cdot \mathrm{yr}\right)$

Climate 5B: $\mathrm{N}=15$

EUI $\left(\mathrm{GJ} / \mathrm{m}^{2} \cdot \mathrm{yr}\right)$

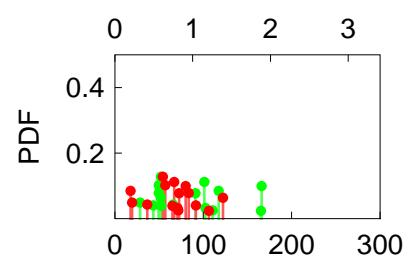

EUI $\left(\mathrm{kBtu} / \mathrm{ft}^{2} \cdot \mathrm{yr}\right)$

Climate 7: $N=10$

EUI $\left(\mathrm{GJ} / \mathrm{m}^{2} \cdot \mathrm{yr}\right)$

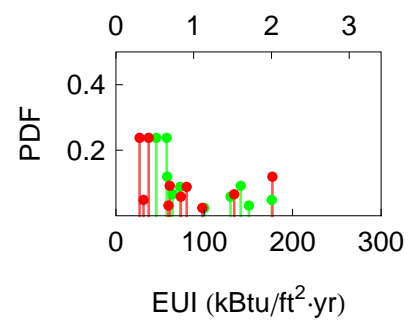

Figure A-10 PDFs for Lodging Buildings in Various Climates:

Survey Results in Red, Model Results in Green 
All Climates: $\mathrm{N}=242$

EUI $\left(\mathrm{GJ} / m^{2} \cdot \mathrm{yr}\right)$

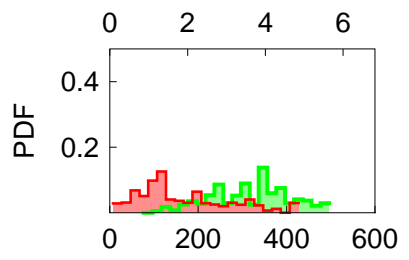

EUI (kBtu/ft $\left.{ }^{2} \cdot y r\right)$

Climate 2B: $\mathrm{N}=0$

Climate 3C: $\mathrm{N}=2$

EUI $\left(\mathrm{GJ} / m^{2} \cdot \mathrm{yr}\right)$

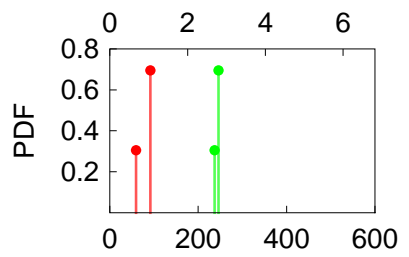

EUI (kBtu/ft $\left.{ }^{2} \cdot y r\right)$

Climate 4C: $\mathrm{N}=2$

EUI $\left(\mathrm{GJ} / \mathrm{m}^{2} \cdot \mathrm{yr}\right)$

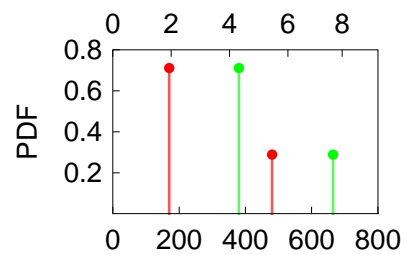

EUI (kBtu/ft $\left.{ }^{2} \cdot y r\right)$

Climate 6A: $\mathrm{N}=28$

EUI (GJ $\left./ m^{2} \cdot y r\right)$

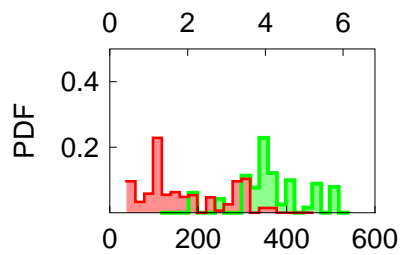

EUI $\left(k B t u / \mathrm{ft}^{2} \cdot \mathrm{yr}\right)$
Climate 1A: $\mathrm{N}=2$

EUI $\left(\mathrm{GJ} / m^{2} \cdot \mathrm{yr}\right)$

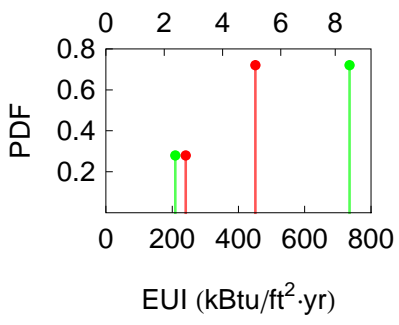

Climate 3A: $\mathrm{N}=27$

EUI $\left(\mathrm{GJ} / \mathrm{m}^{2} \cdot \mathrm{yr}\right)$

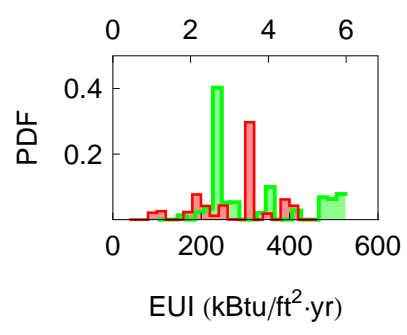

Climate 4A: $N=49$

EUI $\left(\mathrm{GJ} / \mathrm{m}^{2} \cdot \mathrm{yr}\right)$

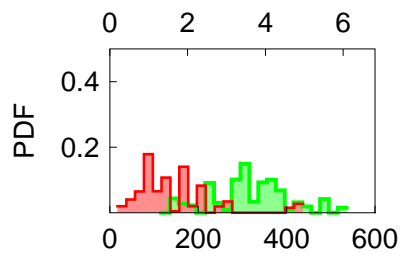

EUI $\left(\mathrm{kBtu} / \mathrm{ft}^{2} \cdot \mathrm{yr}\right)$

Climate 5A: $\mathrm{N}=57$

EUI (GJ $\left./ m^{2} \cdot y r\right)$

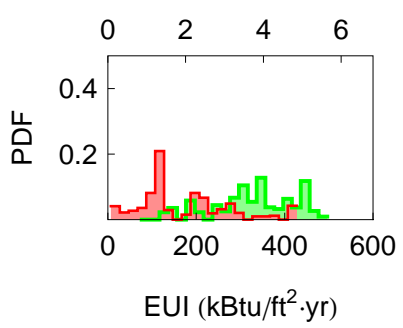

Climate 6B: $\mathrm{N}=2$

EUI $\left(\mathrm{GJ} / \mathrm{m}^{2} \cdot \mathrm{yr}\right)$

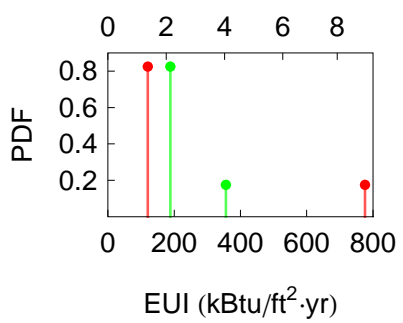

Climate 2A: $\mathrm{N}=34$

EUI $\left(\mathrm{GJ} / m^{2} \cdot \mathrm{yr}\right)$

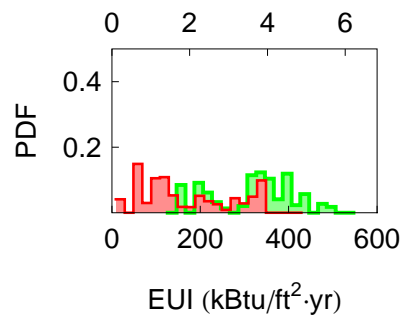

Climate 3B: $\mathrm{N}=22$

EUI $\left(\mathrm{GJ} / m^{2} \cdot \mathrm{yr}\right)$

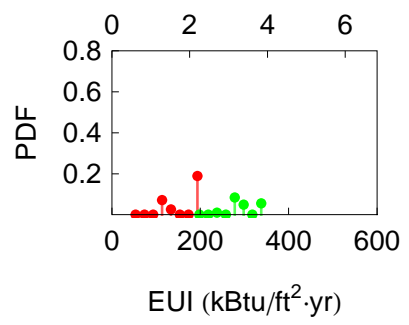

Climate 4B: $\mathrm{N}=0$

Climate 5B: $\mathrm{N}=12$

EUI $\left(\mathrm{GJ} / \mathrm{m}^{2} \cdot \mathrm{yr}\right)$

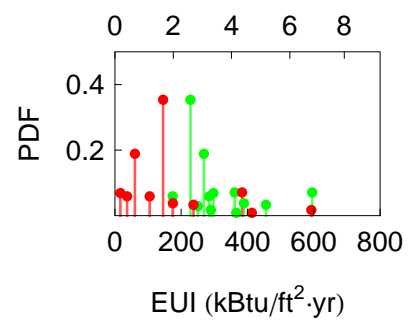

Climate 7: $\mathrm{N}=4$

EUI $\left(\mathrm{GJ} / \mathrm{m}^{2} \cdot \mathrm{yr}\right)$

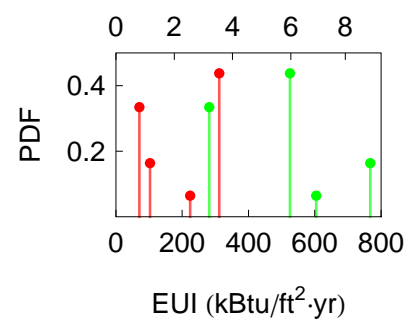

Figure A-11 PDFs for Food Services Buildings in Various Climates:

Survey Results in Red, Model Results in Green 
All Climates: $\mathrm{N}=217$

EUI $\left(\mathrm{GJ} / \mathrm{m}^{2} \cdot \mathrm{yr}\right)$

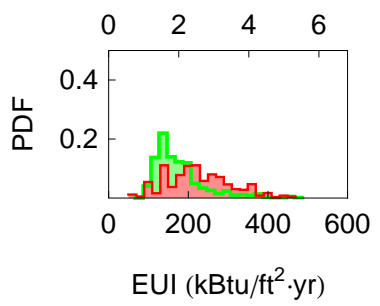

Climate 2B: $\mathrm{N}=3$

EUI $\left(\mathrm{GJ} / \mathrm{m}^{2} \cdot \mathrm{yr}\right)$

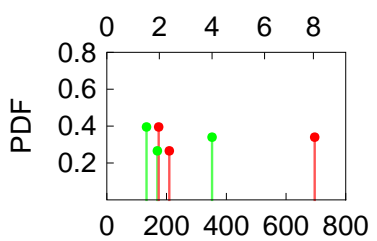

EUI (kBtu/ft $\left.{ }^{2} \cdot y r\right)$

Climate 3C: $\mathrm{N}=5$

EUI $\left(\mathrm{GJ} / m^{2} \cdot \mathrm{yr}\right)$

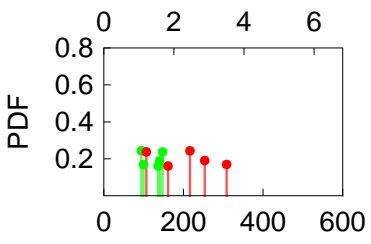

EUI (kBtu/ft $\left.{ }^{2} \cdot y r\right)$

Climate 4C: $N=0$

Climate 6A: $N=21$

EUI $\left(\mathrm{GJ} / \mathrm{m}^{2} \cdot \mathrm{yr}\right)$

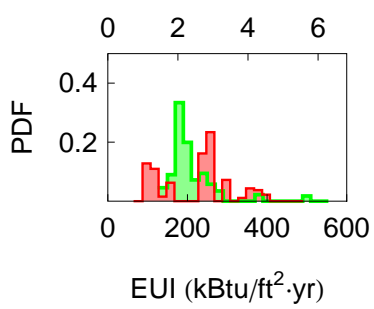

Climate 1A: $\mathrm{N}=2$

EUI $\left(\mathrm{GJ} / \mathrm{m}^{2} \cdot \mathrm{yr}\right)$

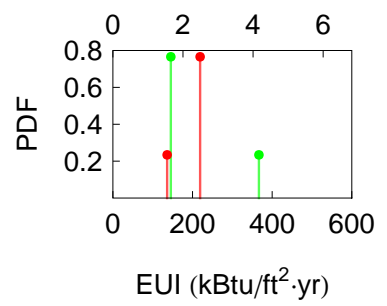

Climate 3A: $\mathrm{N}=19$ EUI $\left(\mathrm{GJ} / \mathrm{m}^{2} \cdot \mathrm{yr}\right)$

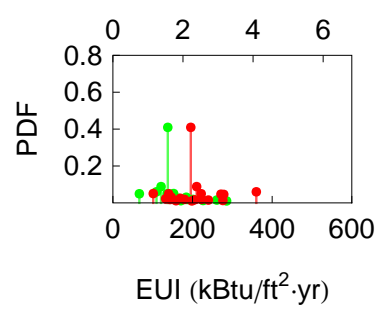

Climate 4A: $\mathrm{N}=45$ EUI (GJ $\left./ m^{2} \cdot y r\right)$

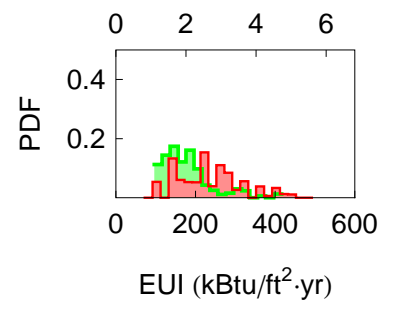

Climate 5A: $N=53$

EUI $\left(\mathrm{GJ} / \mathrm{m}^{2} \cdot \mathrm{yr}\right)$

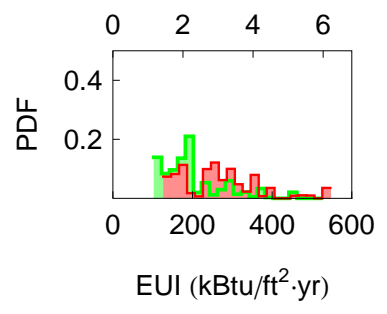

Climate 6B: $\mathrm{N}=6$

EUI (GJ $\left./ m^{2} \cdot y r\right)$

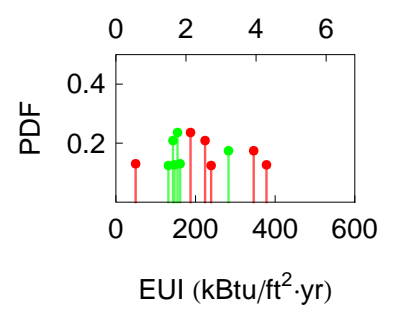

Climate 2A: $\mathrm{N}=25$

EUI $\left(\mathrm{GJ} / m^{2} \cdot \mathrm{yr}\right)$

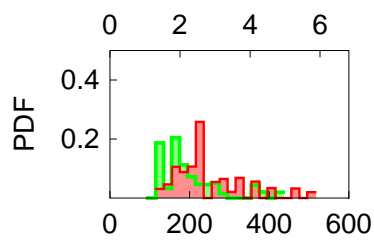

EUI $\left(k B t u / \mathrm{ft}^{2} \cdot \mathrm{yr}\right)$

Climate 3B: $\mathrm{N}=22$

EUI $\left(\mathrm{GJ} / \mathrm{m}^{2} \cdot \mathrm{yr}\right)$

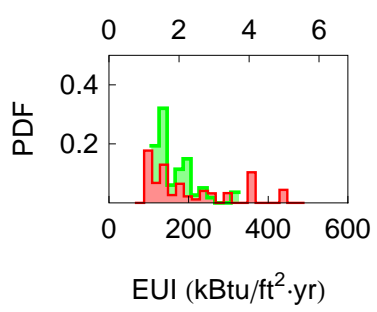

Climate 4B: $\mathrm{N}=2$

EUI $\left(\mathrm{GJ} / \mathrm{m}^{2} \cdot \mathrm{yr}\right)$

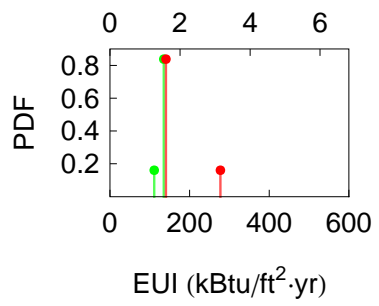

Climate 5B: $\mathrm{N}=12$

EUI $\left(\mathrm{GJ} / \mathrm{m}^{2} \cdot \mathrm{yr}\right)$

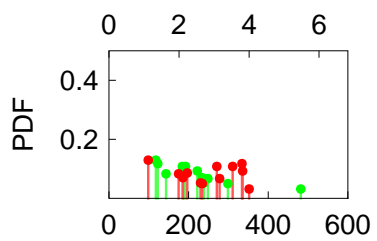

EUI $\left(\mathrm{kBtu} / \mathrm{ft}^{2} \cdot \mathrm{yr}\right)$

Climate 7: $\mathrm{N}=2$

EUI $\left(\mathrm{GJ} / \mathrm{m}^{2} \cdot \mathrm{yr}\right)$

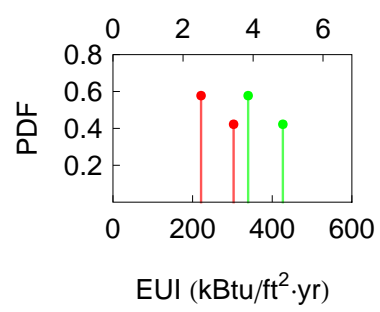

Figure A-12 PDFs for Inpatient Health Care Buildings in Various Climates:

Survey Results in Red, Model Results in Green 


$$
\begin{gathered}
\text { All Climates: } \mathrm{N}=85 \\
\text { EUI }\left(\mathrm{GJ} / \mathrm{m}^{2} \cdot \mathrm{yr}\right)
\end{gathered}
$$

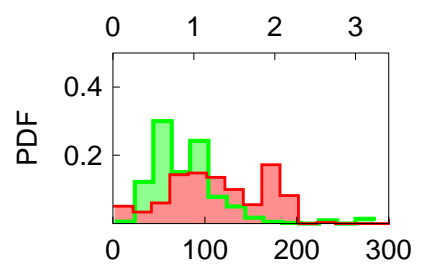

EUI (kBtu/ft $\left.{ }^{2} \cdot y r\right)$

Climate 2B: $\mathrm{N}=0$

Climate 3C: $N=0$

Climate 4C: $\mathrm{N}=0$

Climate 6A: $N=9$

EUI $\left(G J / m^{2} \cdot y r\right)$

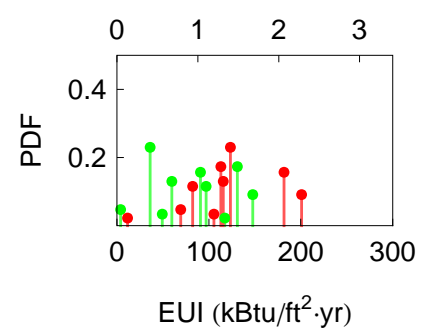

Climate 1A: $N=0$

Climate 3A: $N=4$

EUI $\left(G J / m^{2} \cdot y r\right)$

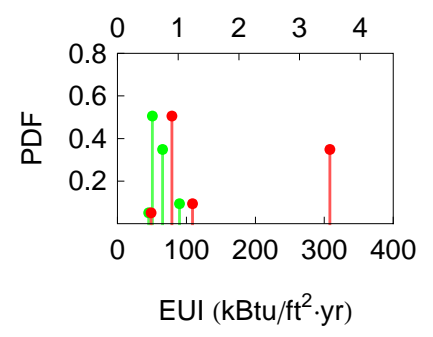

Climate 4A: $N=23$

EUI (GJ $\left./ m^{2} \cdot y r\right)$

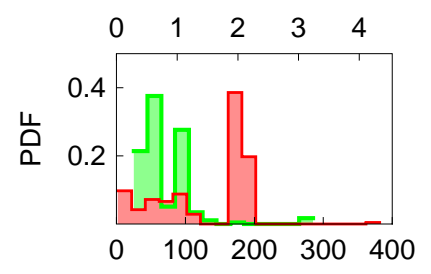

EUI (kBtu/ft' $\left.{ }^{2} \cdot y r\right)$

Climate 5A: $N=22$

EUI (GJ $\left./ m^{2} \cdot y r\right)$

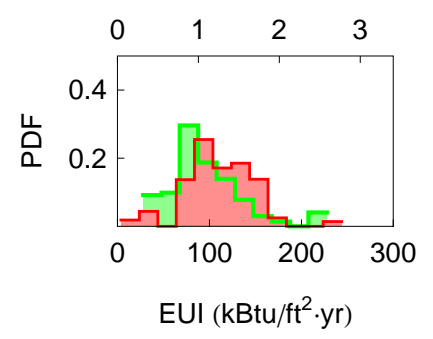

Climate 6B: $\mathrm{N}=3$

EUI $\left(\mathrm{GJ} / \mathrm{m}^{2} \cdot \mathrm{yr}\right)$

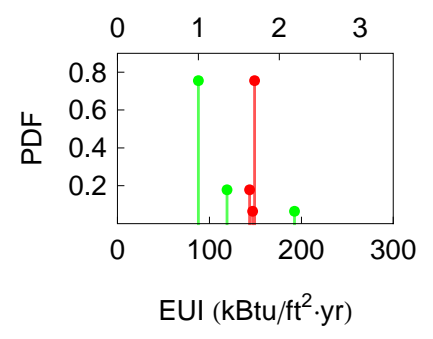

Climate 2A: $\mathrm{N}=7$

EUI (GJ $\left./ m^{2} \cdot y r\right)$

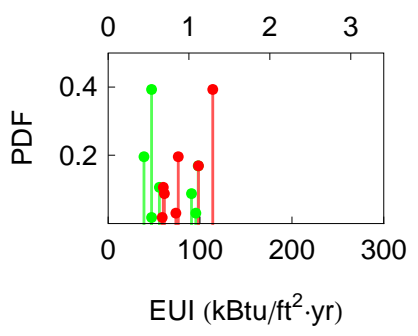

Climate 3B: $\mathrm{N}=4$

EUI $\left(G J / m^{2} \cdot y r\right)$

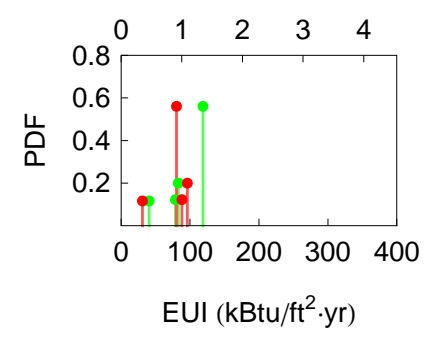

Climate 4B: $\mathrm{N}=0$

Climate 5B: $N=9$

EUI (GJ $\left./ m^{2} \cdot y r\right)$

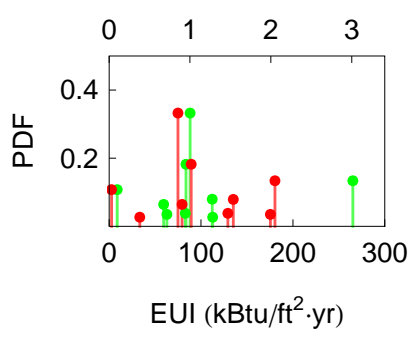

Figure A-13 PDFs for Public Order and Safety Buildings in Various Climates:

Survey Results in Red, Model Results in Green

Climate 7: $\mathrm{N}=0$ 
All Climates: $\mathrm{N}=125$

EUI $\left(\mathrm{GJ} / \mathrm{m}^{2} \cdot \mathrm{yr}\right)$

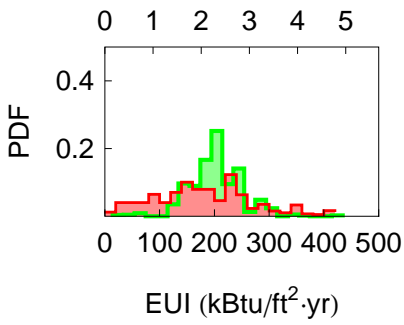

Climate 2B: $\mathrm{N}=0$

Climate 3C: $\mathrm{N}=2$

EUI $\left(\mathrm{GJ} / \mathrm{m}^{2} \cdot \mathrm{yr}\right)$

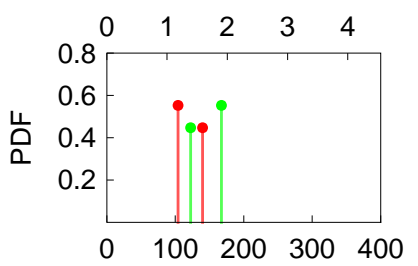

EUI (kBtu/ft² $\cdot y r)$

Climate 4C: $\mathrm{N}=0$

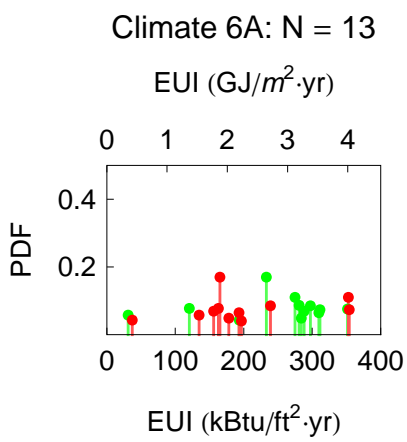

Climate 1A: $N=0$

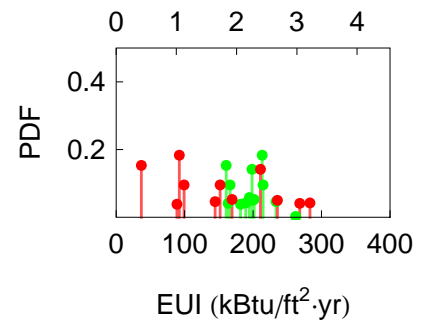

Climate 3A: $N=16$ EUI (GJ $\left./ m^{2} \cdot y r\right)$

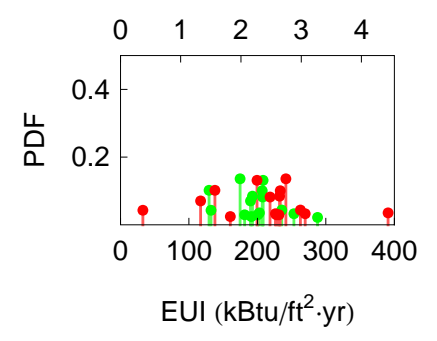

Climate 4A: $N=23$

EUI (GJ $\left./ m^{2} \cdot y r\right)$

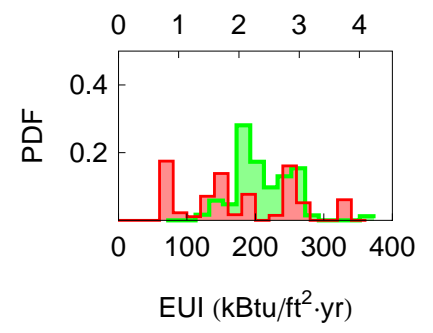

Climate 4B: $N=0$

Climate 3B: $\mathrm{N}=8$ EUI (GJ $\left./ m^{2} \cdot y r\right)$

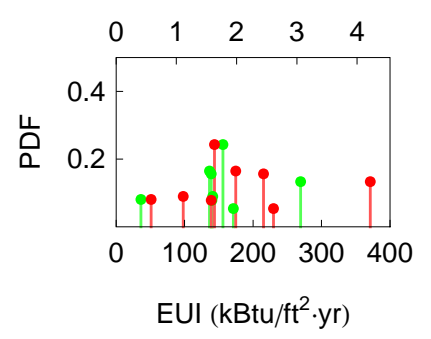

Climate 5B: $\mathrm{N}=8$

EUI $\left(\mathrm{GJ} / \mathrm{m}^{2} \cdot \mathrm{yr}\right)$

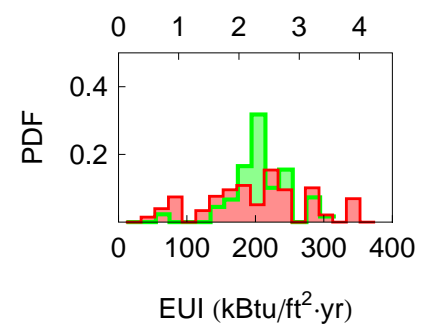

Climate 6B: $\mathrm{N}=0$

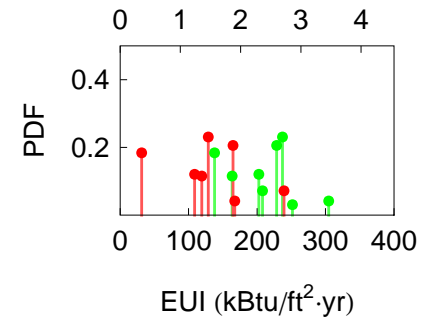

Climate 7: $\mathrm{N}=4$

EUI $\left(\mathrm{GJ} / \mathrm{m}^{2} \cdot \mathrm{yr}\right)$

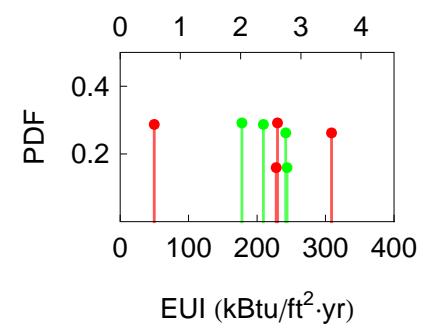

Figure A-14 PDFs for Food Sales Buildings in Various Climates: Survey Results in Red, Model Results in Green 
All Climates: $\mathrm{N}=144$

EUI $\left(\mathrm{GJ} / m^{2} \cdot \mathrm{yr}\right)$

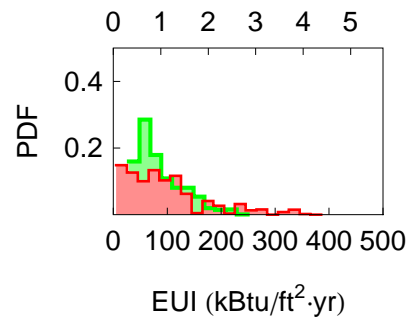

Climate 2B: $\mathrm{N}=0$

Climate 3C: $\mathrm{N}=0$

Climate 4C: $\mathrm{N}=0$

Climate 6A: $\mathrm{N}=12$

EUI $\left(\mathrm{GJ} / \mathrm{m}^{2} \cdot \mathrm{yr}\right)$

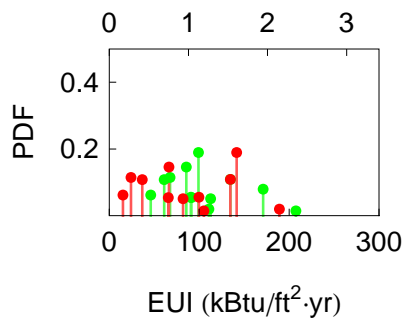

Climate $1 \mathrm{~A}: \mathrm{N}=3$

$\mathrm{EUI}\left(\mathrm{GJ} / m^{2} \cdot \mathrm{yr}\right)$

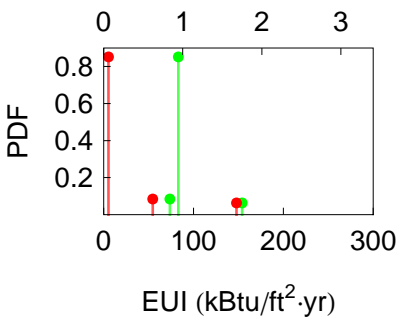

Climate 3A: $N=10$

EUI (GJ $\left./ m^{2} \cdot y r\right)$

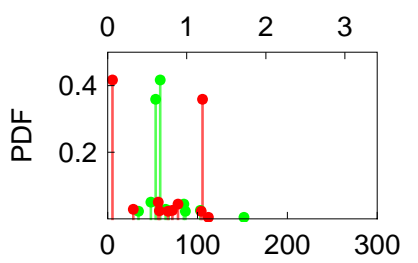

EUI $\left(\mathrm{kBtu} / \mathrm{ft}^{2} \cdot \mathrm{yr}\right)$

Climate 4A: $\mathrm{N}=28$

EUI $\left(\mathrm{GJ} / \mathrm{m}^{2} \cdot \mathrm{yr}\right)$

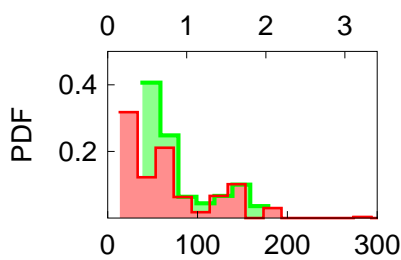

EUI $\left(k B t u / \mathrm{ft}^{2} \cdot \mathrm{yr}\right)$

Climate 5A: $\mathrm{N}=35$

EUI $\left(\mathrm{GJ} / \mathrm{m}^{2} \cdot \mathrm{yr}\right)$

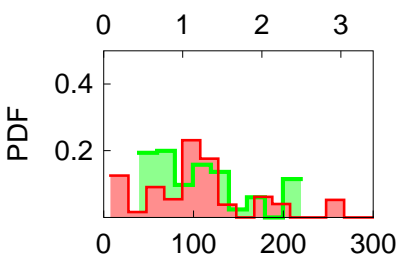

EUI $\left(\mathrm{kBtu} / \mathrm{ft}^{2} \cdot \mathrm{yr}\right)$

Climate 6B: $\mathrm{N}=7$

EUI (GJ $\left./ m^{2} \cdot y r\right)$

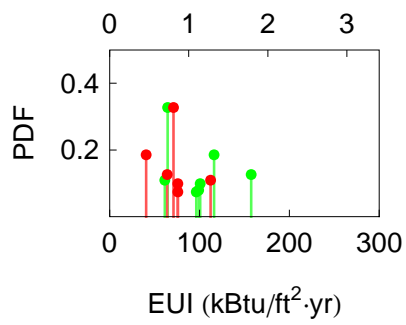

Climate 2A: $N=15$

EUI $\left(\mathrm{GJ} / \mathrm{m}^{2} \cdot \mathrm{yr}\right)$

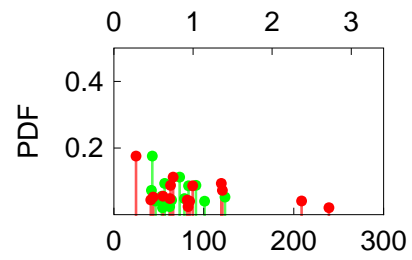

EUI $\left(\mathrm{kBtu} / \mathrm{ft}^{2} \cdot \mathrm{yr}\right)$

Climate 3B: $\mathrm{N}=15$

EUI $\left(\mathrm{GJ} / \mathrm{m}^{2} \cdot \mathrm{yr}\right)$

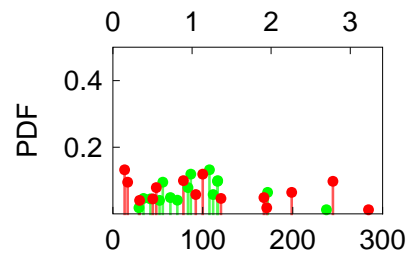

EUI $\left(\mathrm{kBtu} / \mathrm{ft}^{2} \cdot \mathrm{yr}\right)$

Climate 4B: $\mathrm{N}=4$

EUI $\left(\mathrm{GJ} / \mathrm{m}^{2} \cdot \mathrm{yr}\right)$

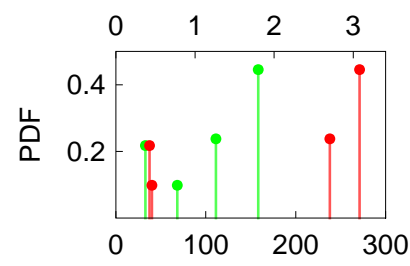

EUI $\left(k B t u / \mathrm{ft}^{2} \cdot \mathrm{yr}\right)$

Climate 5B: $N=10$

EUI $\left(\mathrm{GJ} / \mathrm{m}^{2} \cdot \mathrm{yr}\right)$

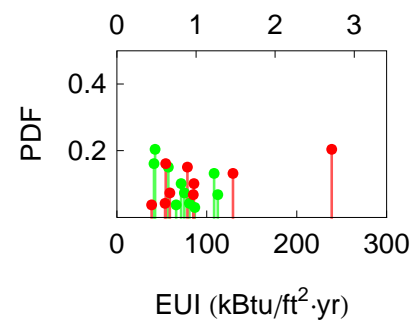

Climate 7: $\mathrm{N}=2$

EUI $\left(\mathrm{GJ} / \mathrm{m}^{2} \cdot \mathrm{yr}\right)$

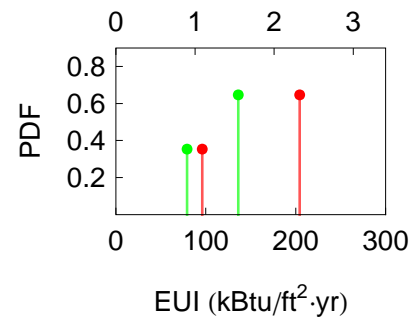

Figure A-15 PDFs for Outpatient Health Care Buildings in Various Climates:

Survey Results in Red, Model Results in Green 
All Climates: $\mathrm{N}=134$

Climate 2A: $\mathrm{N}=17$

EUI $\left(\mathrm{GJ} / \mathrm{m}^{2} \cdot \mathrm{yr}\right)$

EUI $\left(\mathrm{GJ} / m^{2} \cdot \mathrm{yr}\right)$

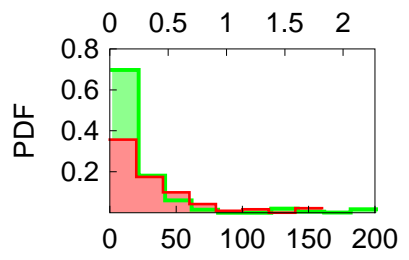

Climate 1A: $N=0$

EUI $\left(k B t u / \mathrm{ft}^{2} \cdot \mathrm{yr}\right)$

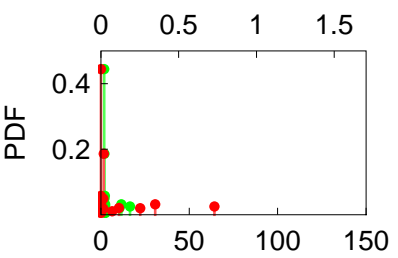

EUI $\left(\mathrm{kBtu} / \mathrm{ft}^{2} \cdot \mathrm{yr}\right)$

Climate 2B: $\mathrm{N}=2$

Climate 3A: $\mathrm{N}=18$

EUI $\left(\mathrm{GJ} / m^{2} \cdot \mathrm{yr}\right)$

EUI (GJ $\left./ m^{2} \cdot y r\right)$
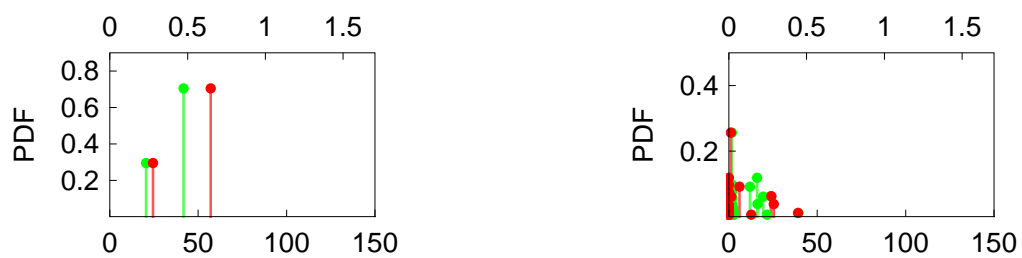

EUI (kBtu/ft $\left.{ }^{2} \cdot y r\right)$

EUI $\left(k B t u / \mathrm{ft}^{2} \cdot \mathrm{yr}\right)$

Climate 3C: $\mathrm{N}=2$

EUI $\left(\mathrm{GJ} / m^{2} \cdot \mathrm{yr}\right)$

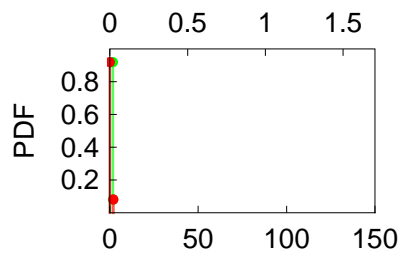

EUI $\left(\mathrm{kBtu} / \mathrm{ft}^{2} \cdot \mathrm{yr}\right)$

Climate 4C: $\mathrm{N}=2$

EUI $\left(\mathrm{GJ} / m^{2} \cdot \mathrm{yr}\right)$

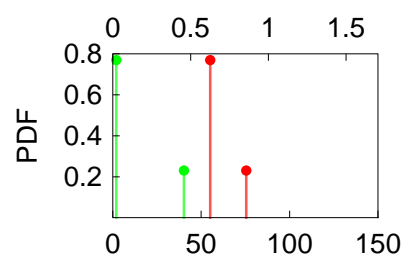

EUI $\left(\mathrm{kBtu} / \mathrm{ft}^{2} \cdot \mathrm{yr}\right)$

Climate 6A: $\mathrm{N}=20$

㟔

Climate 4A: $N=24$

EUI $\left(\mathrm{GJ} / m^{2} \cdot \mathrm{yr}\right)$

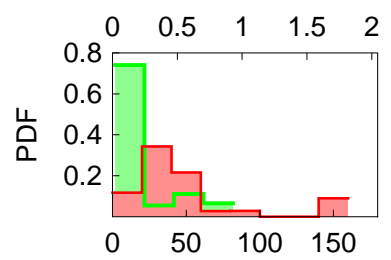

EUI $\left(k B t u / \mathrm{ft}^{2} \cdot \mathrm{yr}\right)$

Climate 5A: $N=29$

EUI $\left(\mathrm{GJ} / \mathrm{m}^{2} \cdot \mathrm{yr}\right)$

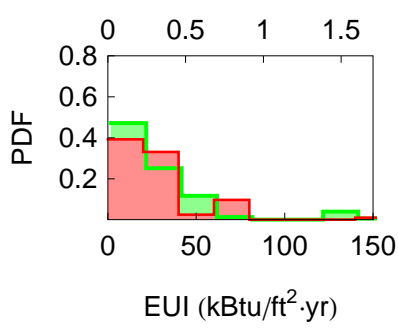

Climate 6B: $\mathrm{N}=0$

Climate 3B: $N=10$

EUI $\left(\mathrm{GJ} / m^{2} \cdot \mathrm{yr}\right)$

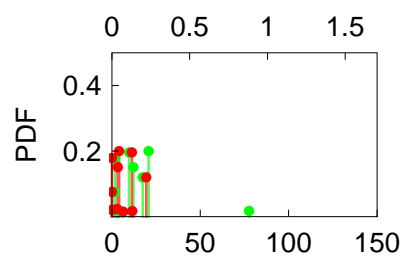

EUI $\left(\mathrm{kBtu} / \mathrm{ft}^{2} \cdot \mathrm{yr}\right)$

Climate 4B: $\mathrm{N}=3$

EUI $\left(\mathrm{GJ} / m^{2} \cdot \mathrm{yr}\right)$

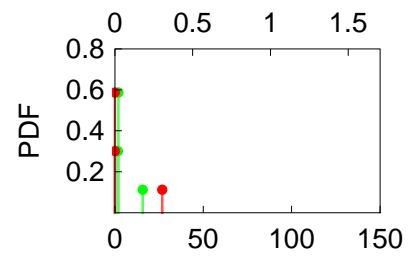

EUI $\left(\mathrm{kBtu} / \mathrm{ft}^{2} \cdot \mathrm{yr}\right)$

Climate 5B: $\mathrm{N}=3$

EUI $\left(\mathrm{GJ} / \mathrm{m}^{2} \cdot \mathrm{yr}\right)$

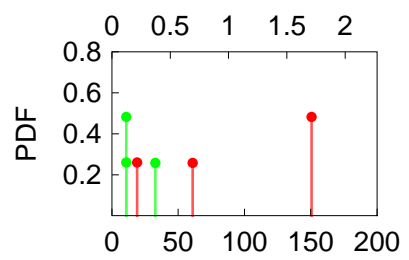

EUI $\left(\mathrm{kBtu} / \mathrm{ft}^{2} \cdot \mathrm{yr}\right)$

Climate 7: $\mathrm{N}=3$

EUI $\left(\mathrm{GJ} / m^{2} \cdot \mathrm{yr}\right)$

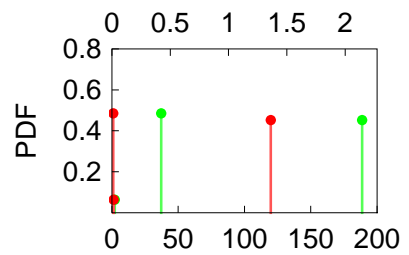

EUI $\left(\mathrm{kBtu} / \mathrm{ft}^{2} \cdot \mathrm{yr}\right)$

Figure A-16 PDFs for Vacant Buildings in Various Climates:

Survey Results in Red, Model Results in Green 
All Climates: $\mathrm{N}=64$

EUI $\left(\mathrm{GJ} / \mathrm{m}^{2} \cdot \mathrm{yr}\right)$

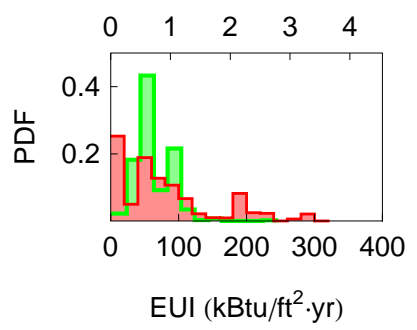

Climate 2B: $\mathrm{N}=0$

Climate 3C: $\mathrm{N}=0$

Climate 4C: $\mathrm{N}=0$

Climate 6A: $N=8$

EUI $\left(\mathrm{GJ} / \mathrm{m}^{2} \cdot \mathrm{yr}\right)$

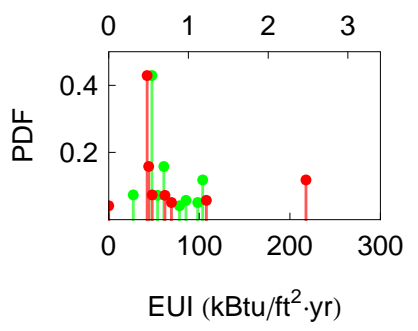

Climate $1 \mathrm{~A}: \mathrm{N}=0$

Climate 3A: $\mathrm{N}=3$

EUI (GJ $\left./ m^{2} \cdot y r\right)$

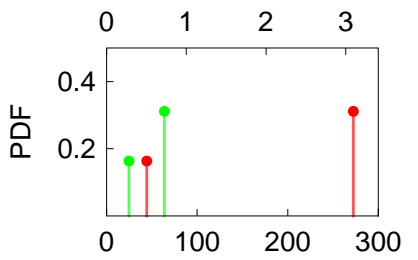

EUI $\left(\mathrm{kBtu} / \mathrm{ft}^{2} \cdot \mathrm{yr}\right)$

Climate 4A: $N=10$

EUI $\left(\mathrm{GJ} / \mathrm{m}^{2} \cdot \mathrm{yr}\right)$

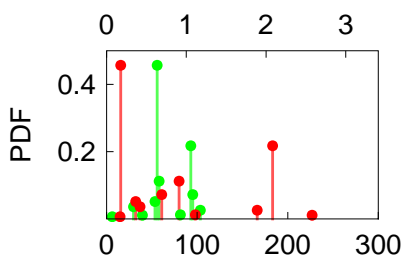

EUI $\left(k B t u / \mathrm{ft}^{2} \cdot \mathrm{yr}\right)$

Climate 5A: $N=21$

EUI $\left(\mathrm{GJ} / \mathrm{m}^{2} \cdot \mathrm{yr}\right)$

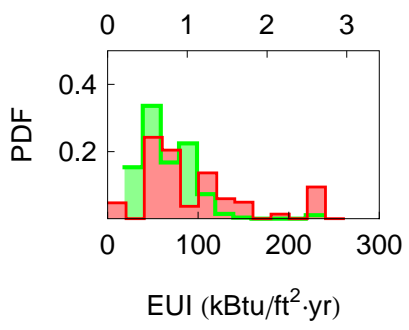

Climate 6B: $\mathrm{N}=2$

EUI $\left(\mathrm{GJ} / \mathrm{m}^{2} \cdot \mathrm{yr}\right)$

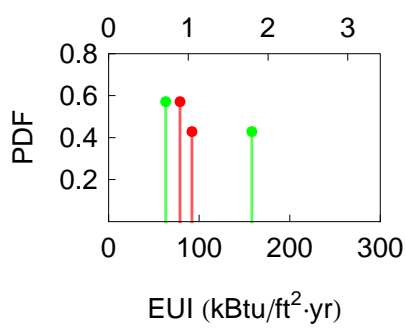

Climate 2A: $N=6$

EUI $\left(\mathrm{GJ} / m^{2} \cdot \mathrm{yr}\right)$

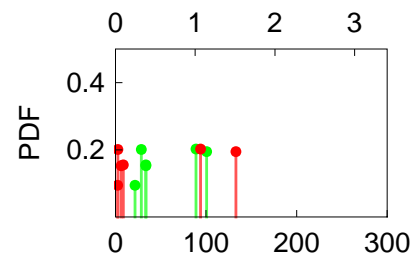

EUI (kBtu/ft $\left.{ }^{2} \cdot y r\right)$

Climate 3B: $N=4$

EUI (GJ $\left./ m^{2} \cdot y r\right)$

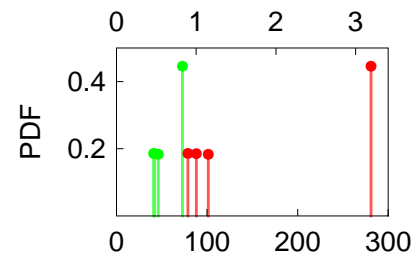

EUI $\left(k B t u / \mathrm{ft}^{2} \cdot \mathrm{yr}\right)$

Climate 4B: $\mathrm{N}=2$

EUI $\left(\mathrm{GJ} / \mathrm{m}^{2} \cdot \mathrm{yr}\right)$

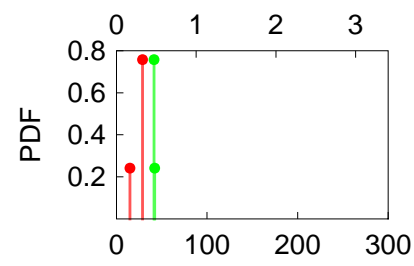

EUI (kBtu/ft $\left.{ }^{2} \cdot y r\right)$

Climate 5B: $N=4$

EUI $\left(\mathrm{GJ} / \mathrm{m}^{2} \cdot \mathrm{yr}\right)$

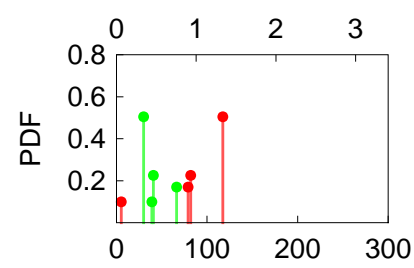

EUI $\left(k B t u / \mathrm{ft}^{2} \cdot \mathrm{yr}\right)$

Climate 7: $N=4$

EUI $\left(\mathrm{GJ} / \mathrm{m}^{2} \cdot \mathrm{yr}\right)$

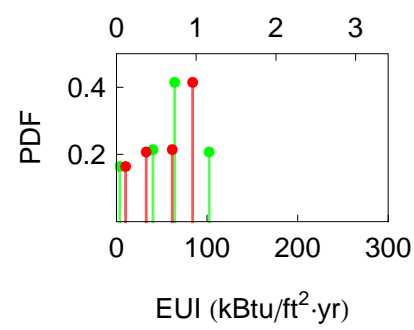

Figure A-17 PDFs for Other Buildings in Various Climates:

Survey Results in Red, Model Results in Green 

All Climates: $\mathrm{N}=73$
EUI (GJ $/ m^{2} \cdot$ yr)

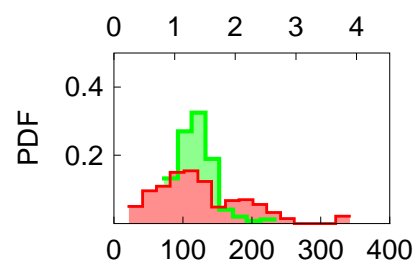

EUI (kBtu/ft $\left.{ }^{2} \cdot y r\right)$

Climate 2B: $\mathrm{N}=0$

Climate 3C: $\mathrm{N}=0$

Climate 4C: $\mathrm{N}=0$

Climate 6A: $N=8$

EUI $\left(\mathrm{GJ} / \mathrm{m}^{2} \cdot \mathrm{yr}\right)$

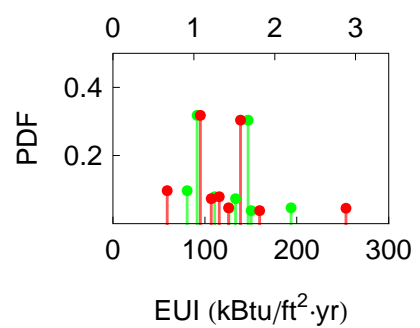

Climate $1 \mathrm{~A}: \mathrm{N}=0$

Climate 3A: $N=5$

EUI $\left(G J / m^{2} \cdot y r\right)$

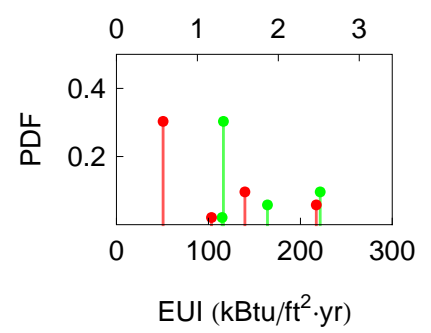

Climate 4A: $\mathrm{N}=16$

EUI (GJ $\left./ m^{2} \cdot y r\right)$

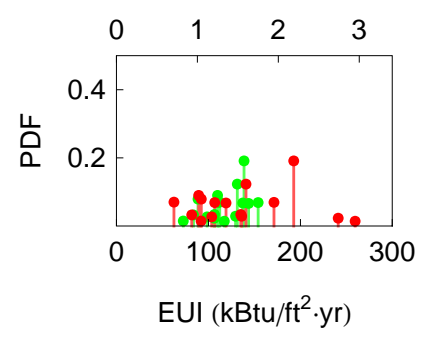

Climate 5A: $N=22$

EUI $\left(\mathrm{GJ} / \mathrm{m}^{2} \cdot \mathrm{yr}\right)$

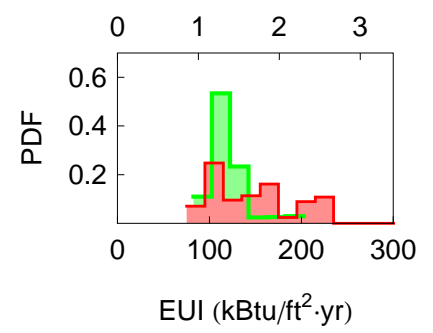

Climate 6B: $\mathrm{N}=3$

EUI (GJ $\left./ m^{2} \cdot y r\right)$

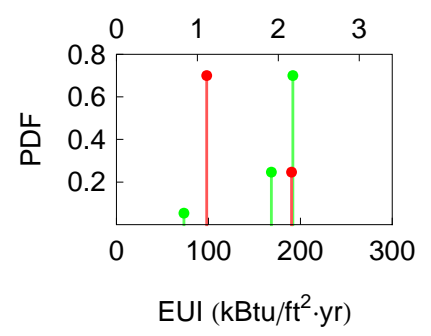

Climate 2A: $\mathrm{N}=5$

EUI (GJ $\left./ m^{2} \cdot y r\right)$

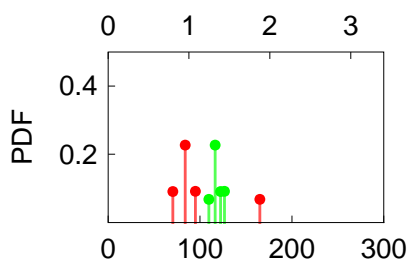

EUI (kBtu/ft' $\cdot$.yr)

Climate 3B: $\mathrm{N}=7$

EUI $\left(G J / m^{2} \cdot y r\right)$

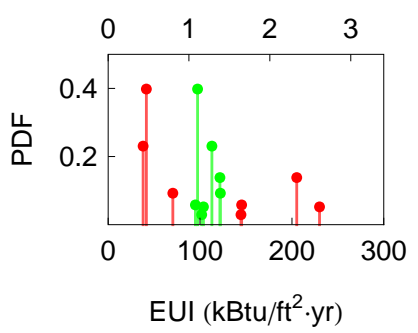

Climate 4B: $N=0$

Climate 5B: $N=6$

EUI (GJ $\left./ m^{2} \cdot y r\right)$

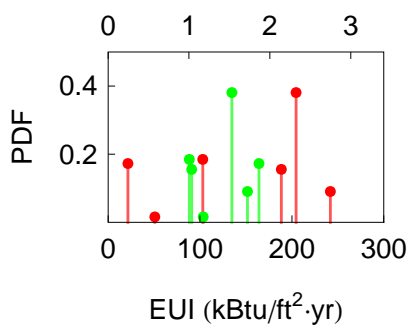

Climate 7: $\mathrm{N}=0$

Figure A-18 PDFs for Skilled Nursing Buildings in Various Climates:

Survey Results in Red, Model Results in Green 


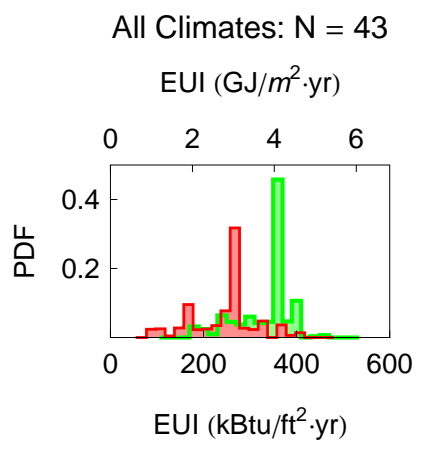

Climate 1A: $N=0$

Climate 3A: $\mathrm{N}=2$

EUI $\left(\mathrm{GJ} / \mathrm{m}^{2} \cdot \mathrm{yr}\right)$

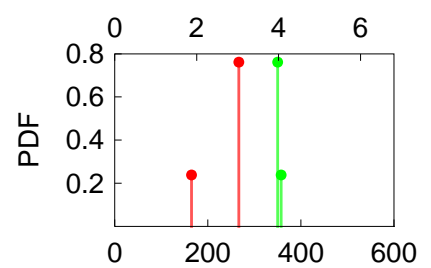

EUI (kBtu/ft $\left.{ }^{2} \cdot y r\right)$

Climate 4A: $\mathrm{N}=5$

EUI $\left(\mathrm{GJ} / m^{2} \cdot \mathrm{yr}\right)$

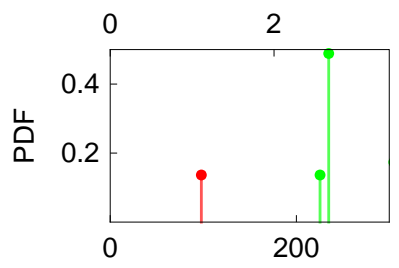

EUI $\left(\mathrm{kBtu} / \mathrm{ft}^{2} \cdot \mathrm{yr}\right)$

Climate 5A: $N=19$

EUI (GJ $\left./ m^{2} \cdot y r\right)$

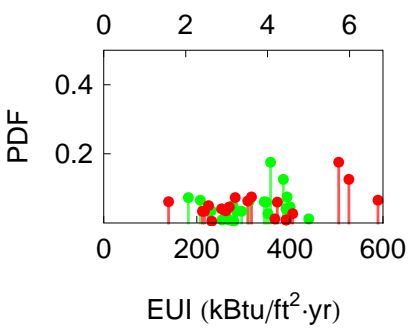

Climate 6B: $\mathrm{N}=2$

EUI $\left(\mathrm{GJ} / \mathrm{m}^{2} \cdot \mathrm{yr}\right)$

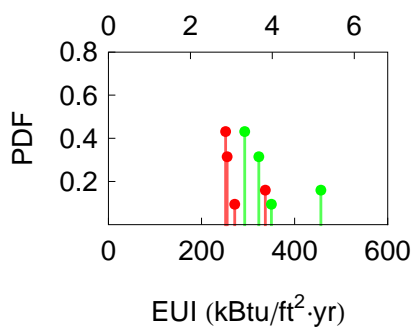

$\mathrm{EUI}\left(\mathrm{GJ} / m^{2} \cdot \mathrm{yr}\right)$

Climate 4C: $\mathrm{N}=0$

(a)

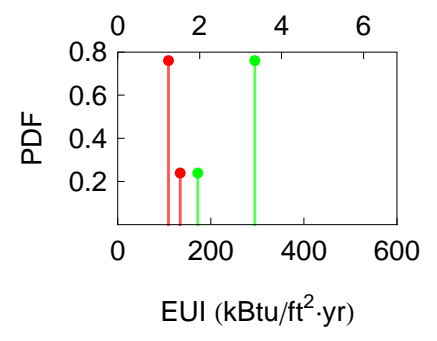

Figure A-19 PDFs for Laboratory Buildings in Various Climates: Survey Results in Red, Model Results in Green

Climate 3B: $\mathrm{N}=8$ $\mathrm{EUI}\left(\mathrm{GJ} / m^{2} \cdot \mathrm{yr}\right)$

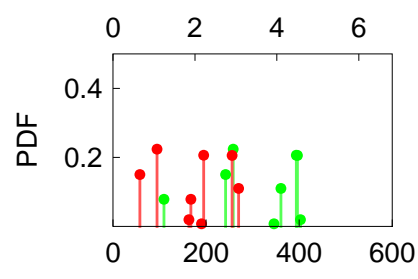

EUI $\left(\mathrm{kBtu} / \mathrm{ft}^{2} \cdot \mathrm{yr}\right)$

Climate 4B: $N=0$

Climate 7: $\mathrm{N}=0$ 
All Climates: $\mathrm{N}=20$

EUI $\left(\mathrm{GJ} / m^{2} \cdot \mathrm{yr}\right)$

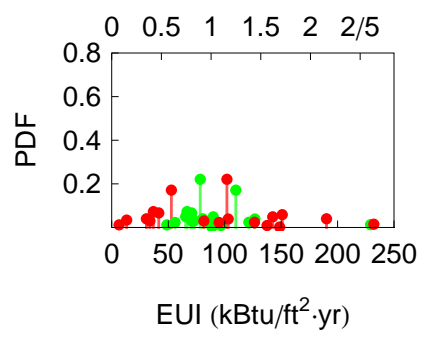

Climate 2B: $\mathrm{N}=0$

Climate 3C: $\mathrm{N}=0$

Climate 4C: $N=0$
Climate 3A: $N=0$

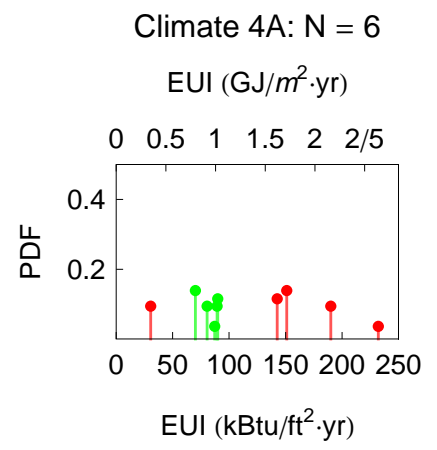

Climate 5A: $N=5$ EUI (GJ $\left./ m^{2} \cdot y r\right)$

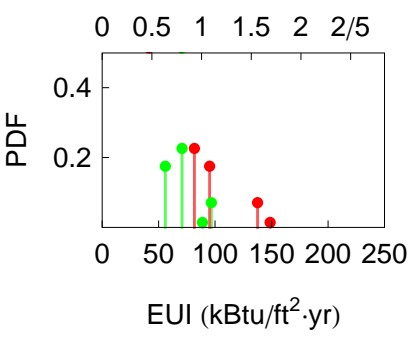

Climate 6B: $\mathrm{N}=0$

Climate 6A: $\mathrm{N}=3$

EUI $\left(\mathrm{GJ} / \mathrm{m}^{2} \cdot \mathrm{yr}\right)$

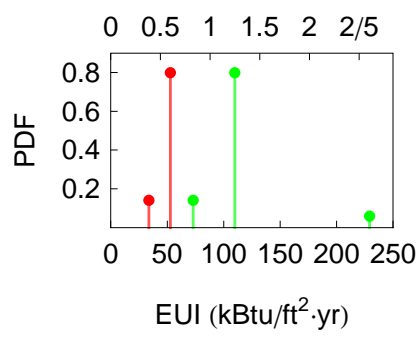

Climate 3B: $\mathrm{N}=0$

Climate 4B: $\mathrm{N}=0$

$$
\begin{gathered}
\text { Climate 5B: } \mathrm{N}=3 \\
\text { EUI }\left(\mathrm{GJ} / \mathrm{m}^{2} \cdot \mathrm{yr}\right)
\end{gathered}
$$

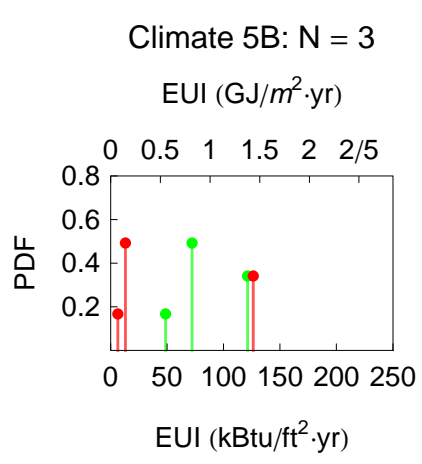

Climate 2A: $\mathrm{N}=0$

Figure A-20 PDFs for Refrigerated Warehouse Buildings in Various Climates: Survey Results in Red, Model Results in Green 


\section{A.4 Scatter Plots}

The results for individual sample buildings can also be compared. The models are generated from random assignments for many parameters that will affect energy performance. Therefore, compared to aggregations of large numbers, there is little reason to expect that the models and survey results should agree well. There is also concern that the rounding of floor areas in CBECS data for anonymity will introduce errors in metrics that are normalized by floor area. Nevertheless, the trends on a one-for-one basis are interesting to see, so this section presents a long series of X-Y scatter plots that compare total EUI with individual points for each sample. The 2003 CBECS survey results are on the X-axis; the modeling results are on the Y-axis. Figure A-21 through Figure A-39 present scatter plots by subsector. Figure A-40 through Figure A-53 present them by climate zone classification. 


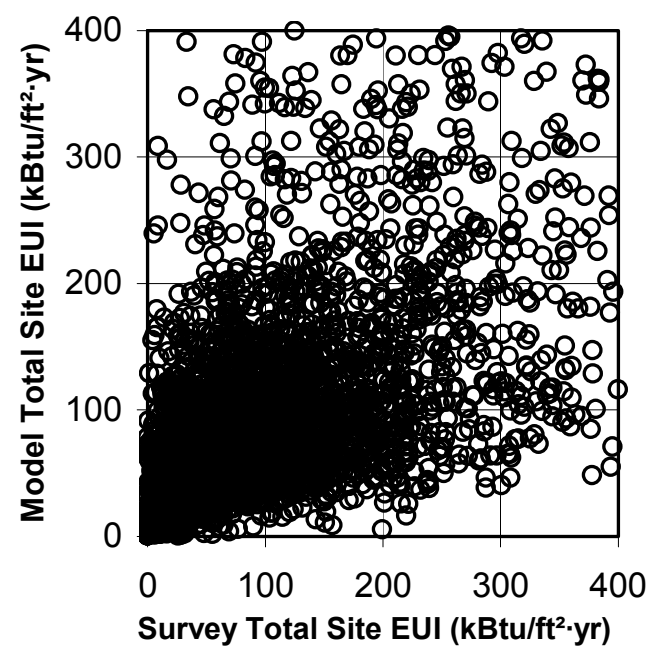

Figure A-21 Scatter Plot of Total EUI: All Buildings

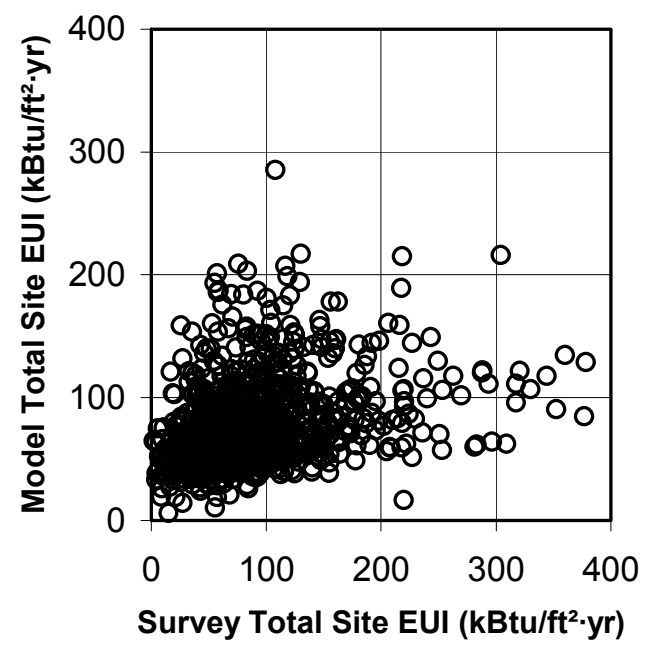

Figure A-22 Scatter Plot of Total EUI: Offices

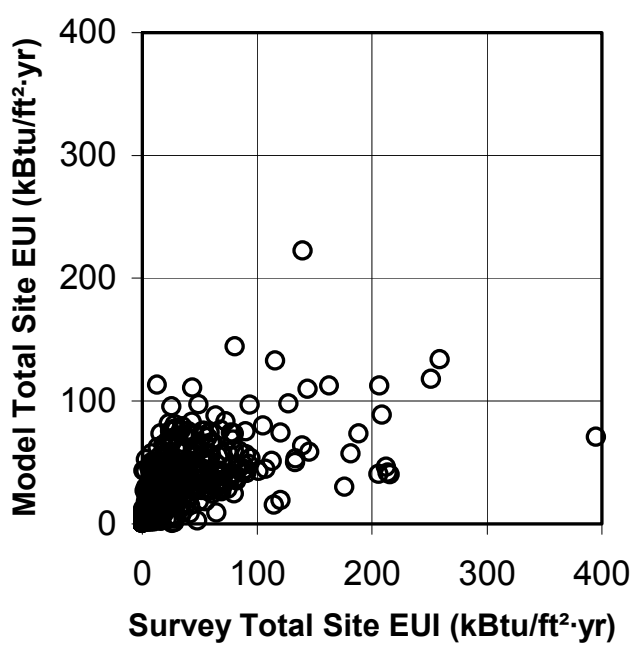

Figure A-23 Scatter Plot of Total EUI: Nonrefrigerated Warehouse

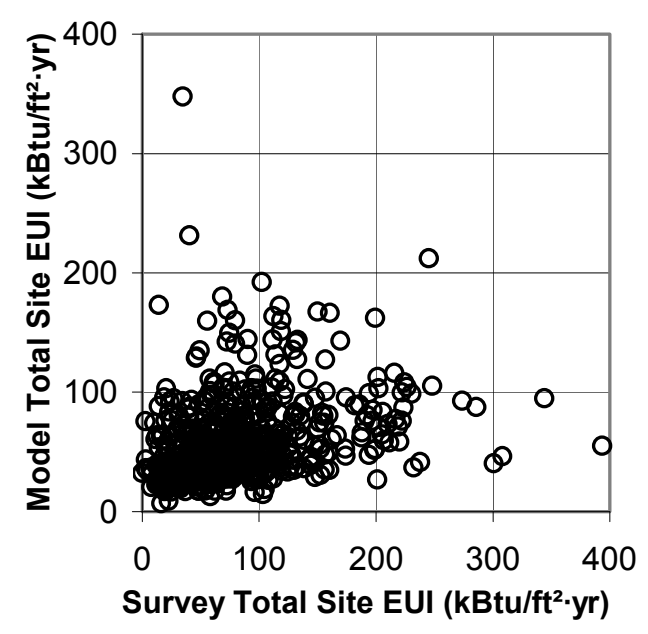

Figure A-24 Scatter Plot of Total EUI: Education 


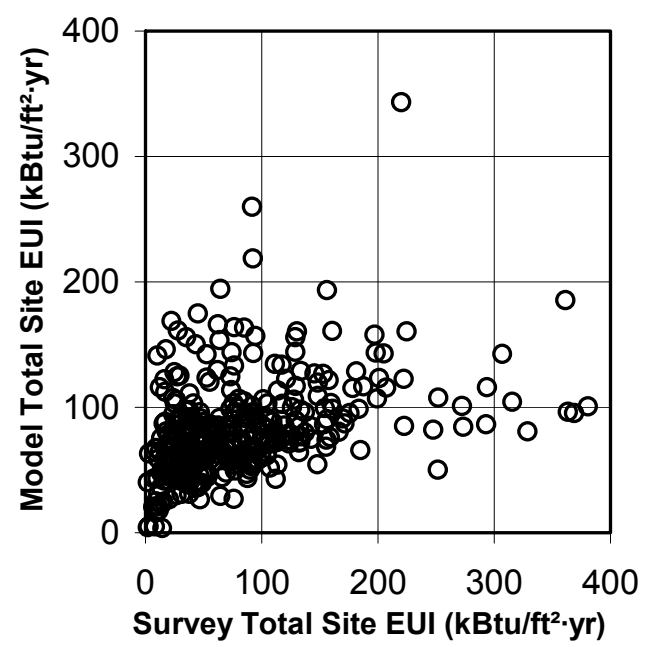

Figure A-25 Scatter Plot of Total EUI: Retail (except Malls)

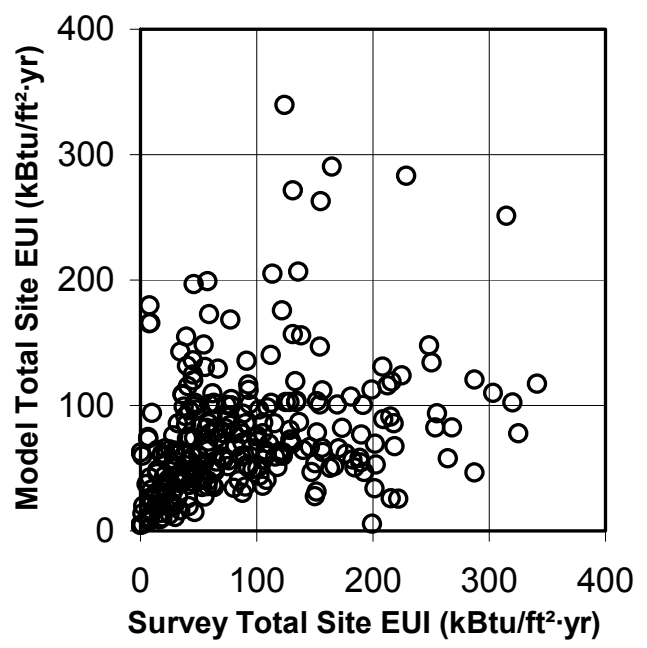

Figure A-26 Scatter Plot of Total EUI: Public Assembly

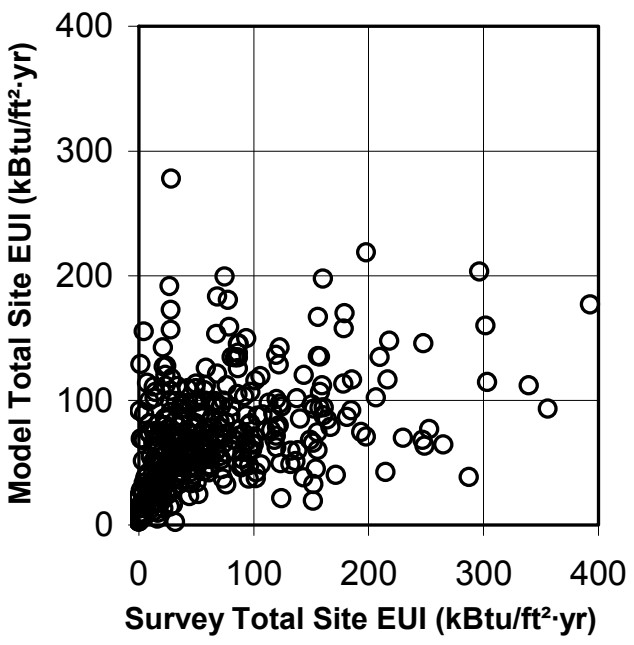

Figure A-27 Scatter Plot of Total EUI: Service

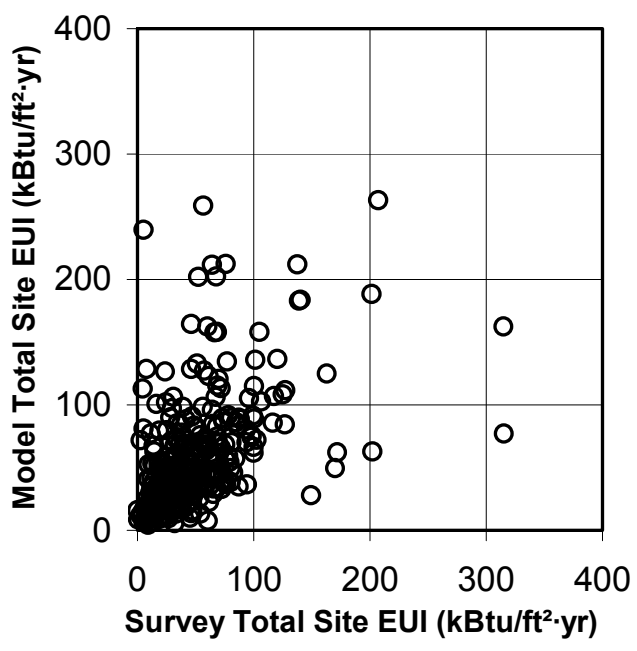

Figure A-28 Scatter Plot of Total EUI: Religious Worship 


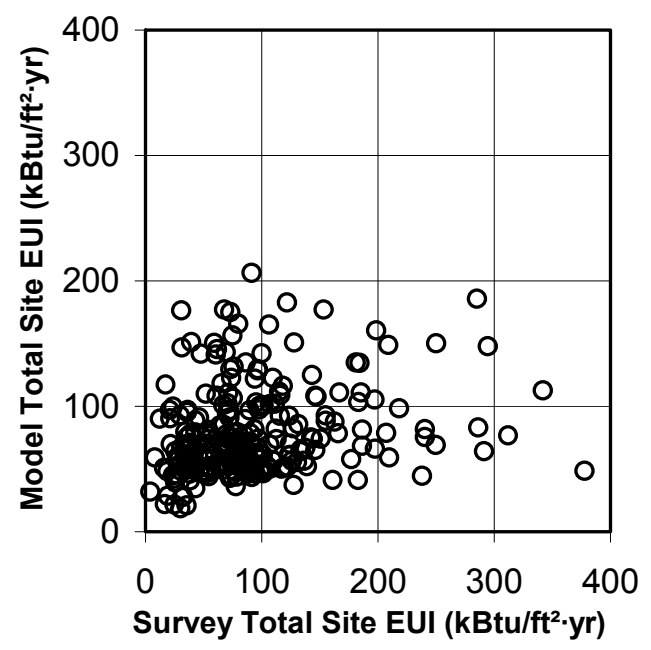

Figure A-29 Scatter Plot of Total EUI: Lodging

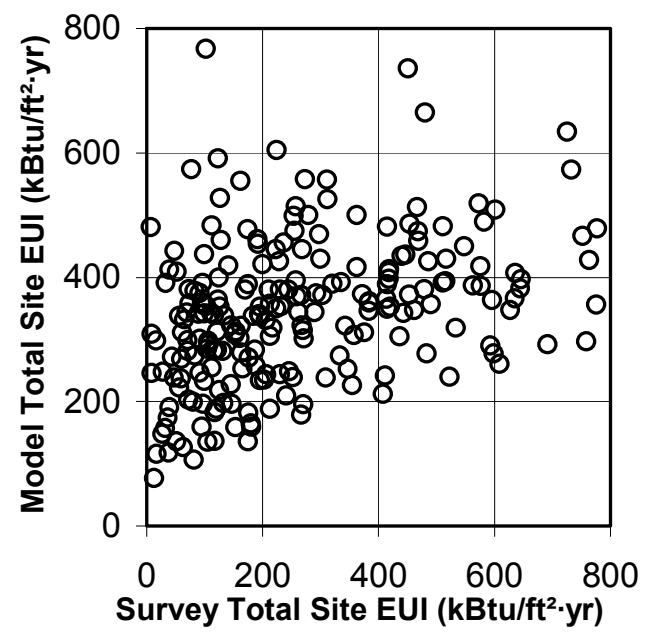

Figure A-30 Scatter Plot of Total EUI: Food Service

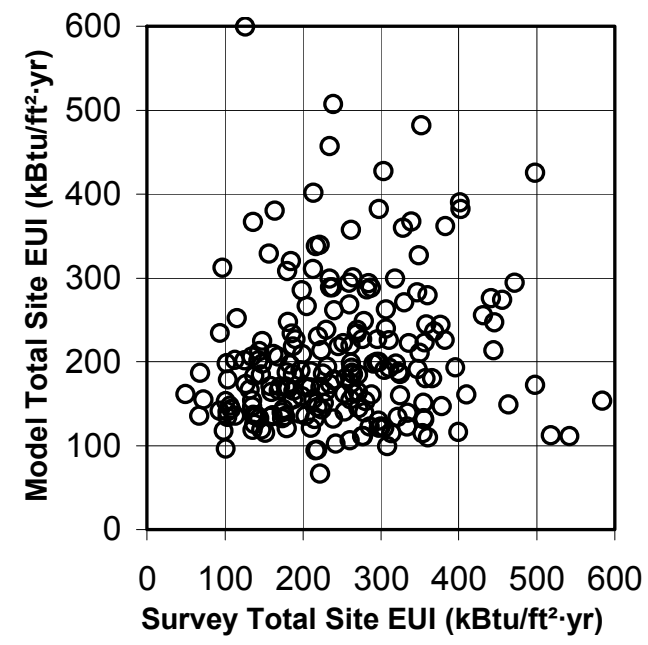

Figure A-31 Scatter Plot of Total EUI: Health Care (Inpatient)

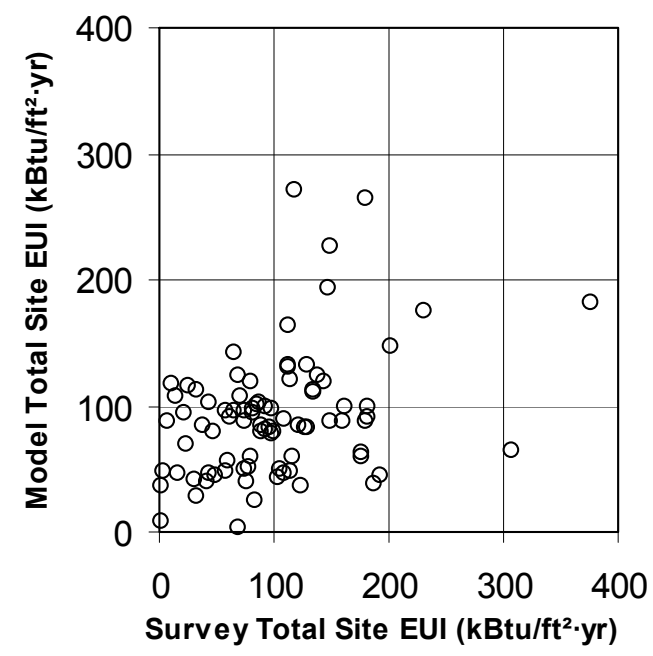

Figure A-32 Scatter Plot of Total EUI: Public Order and Safety 


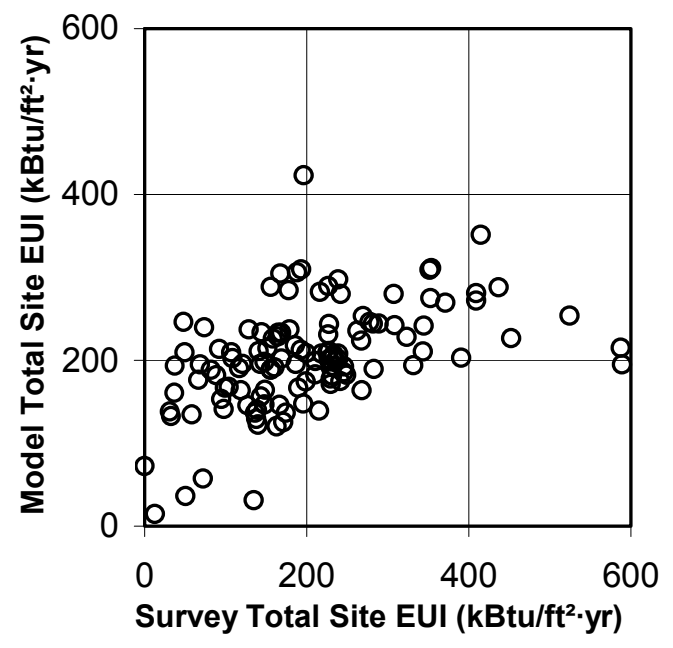

Figure A-33 Scatter Plot of Total EUI: Food Sales

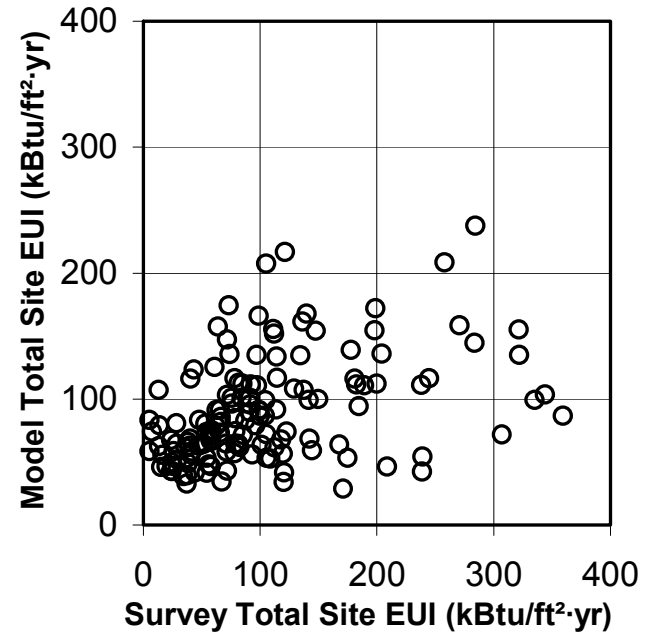

Figure A-34 Scatter Plot of Total EUI: Health Care (Outpatient)

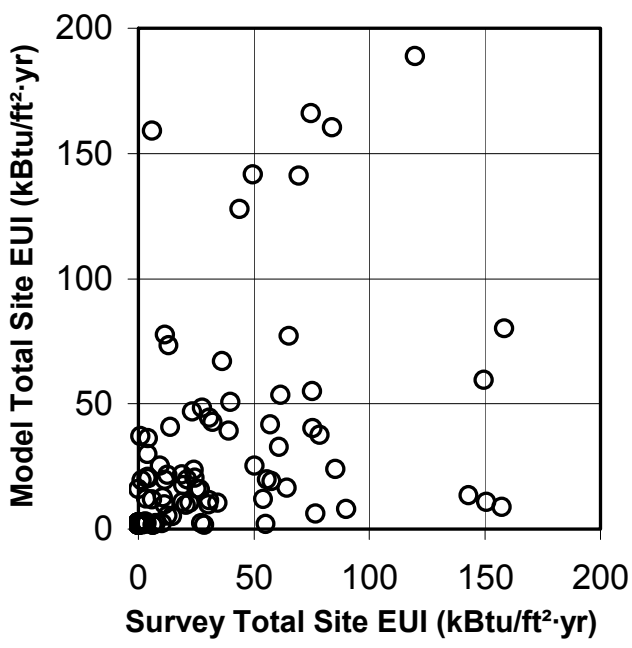

Figure A-35 Scatter Plot of Total EUI: Vacant

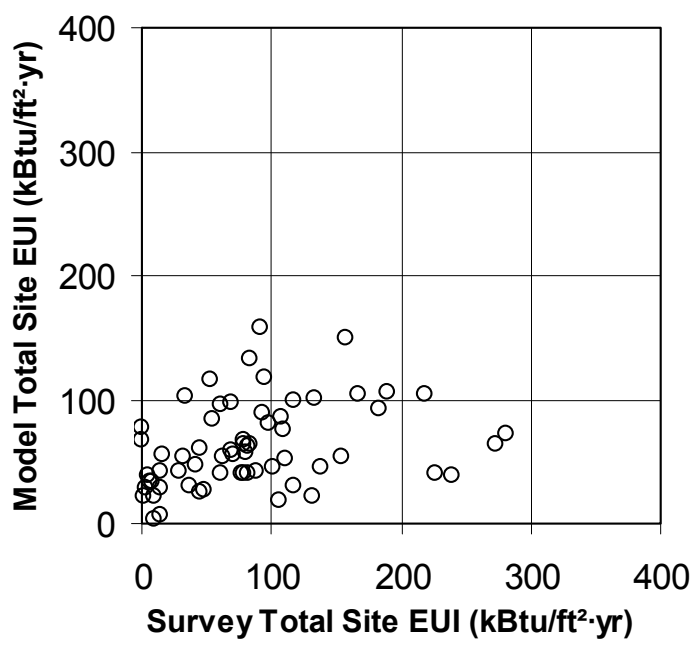

Figure A-36 Scatter Plot of Total EUI: Other 


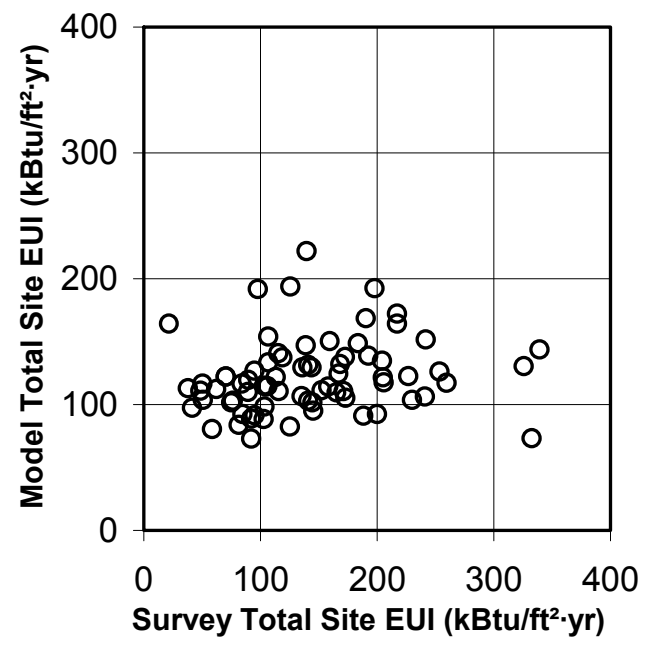

Figure A-37 Scatter Plot of Total EUI: Skilled Nursing

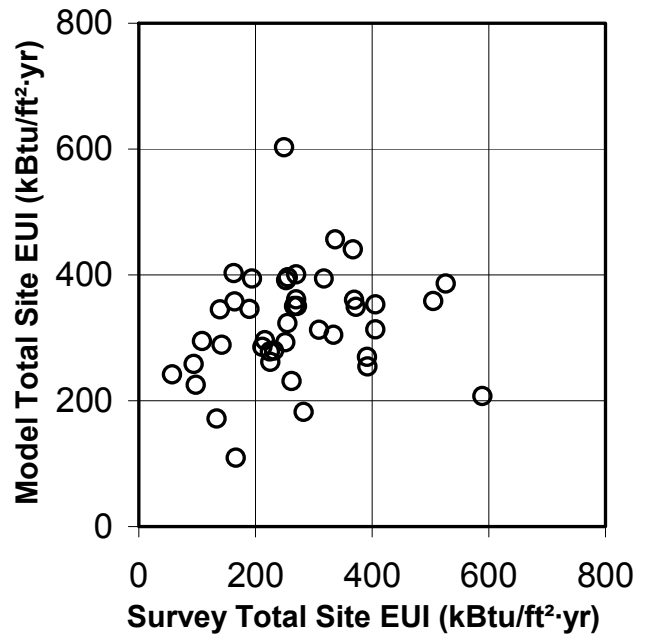

Figure A-38 Scatter Plot of Total EUI: Laboratory

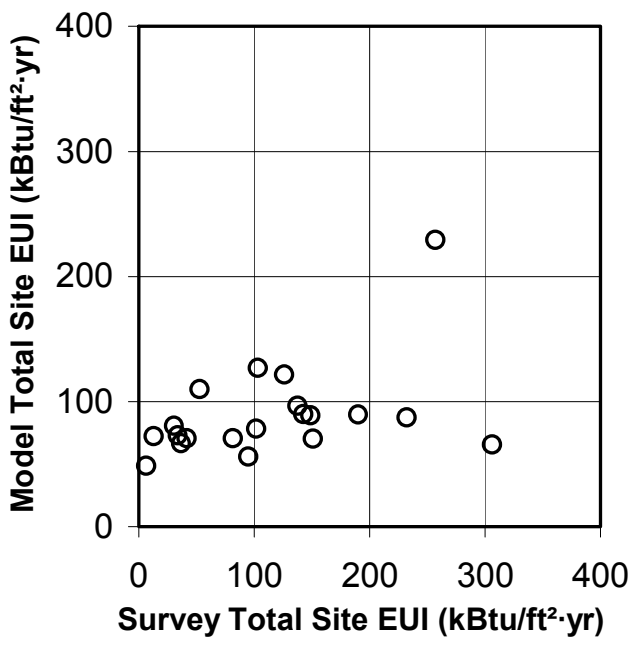

Figure A-39 Scatter Plot of Total EUI: Refrigerated Warehouse

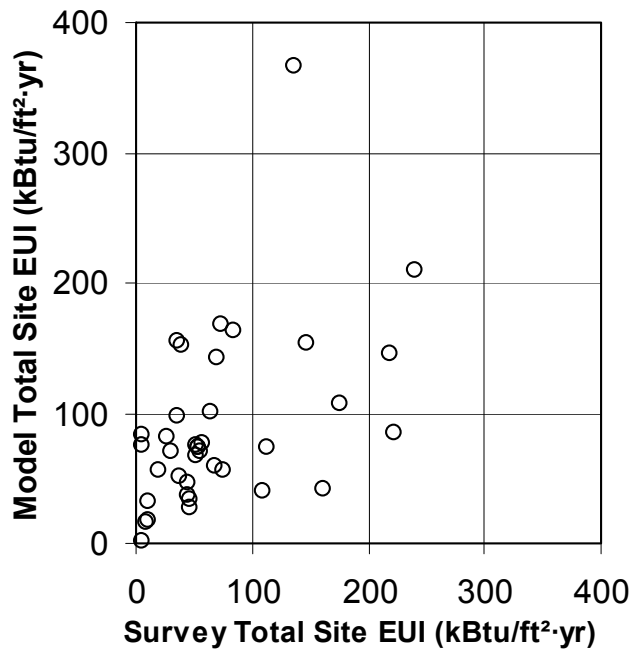

Figure A-40 Scatter Plot of Total EUI: Climate Zone 1A 


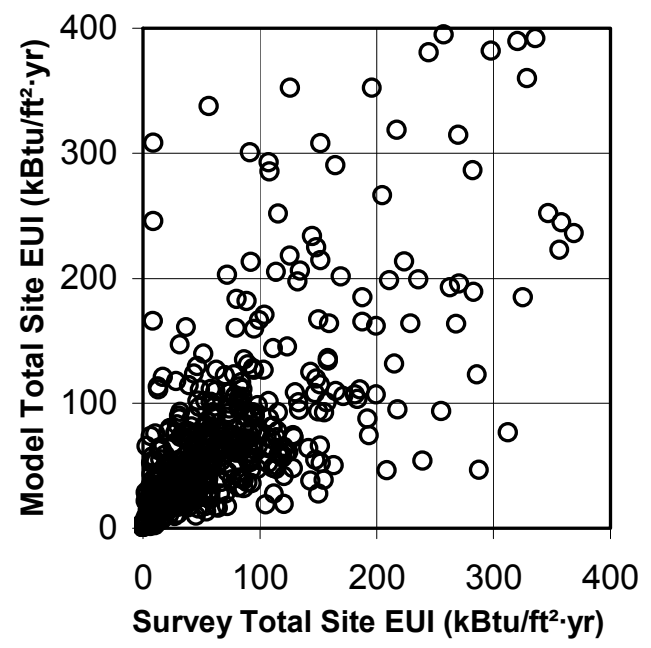

Figure A-41 Scatter Plot of Total EUI: Climate Zone 2A

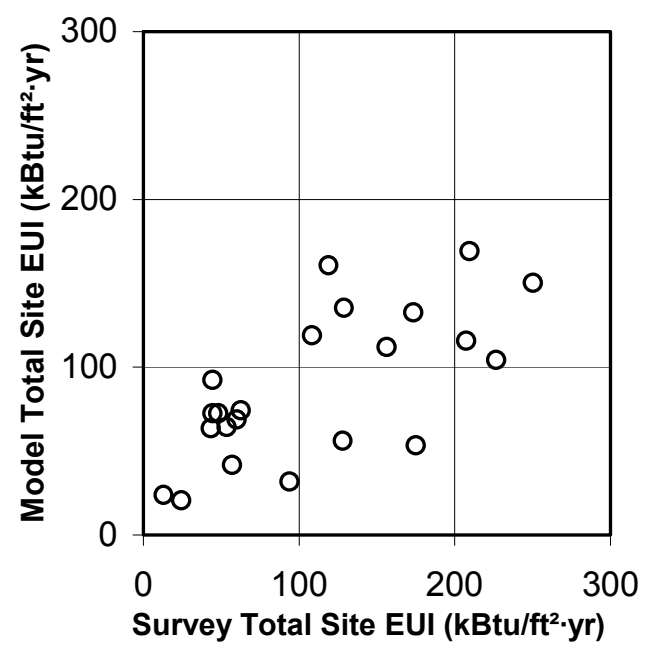

Figure A-42 Scatter Plot of Total EUI: Climate Zone 2B

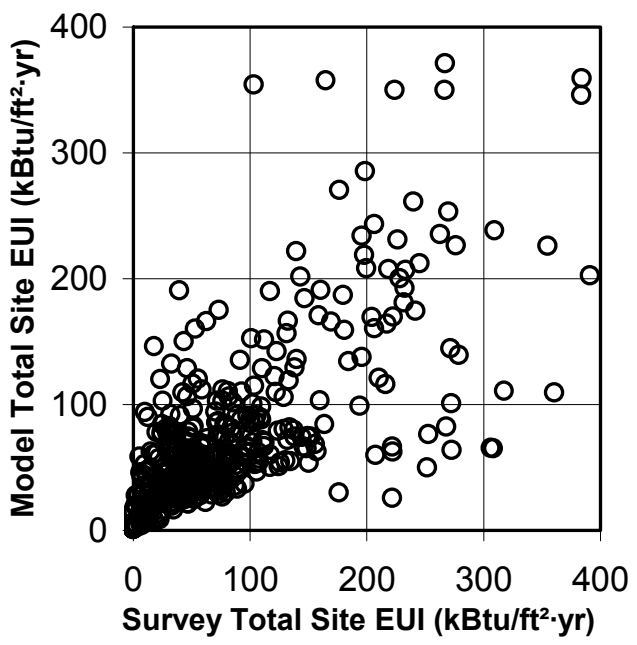

Figure A-43 Scatter Plot of Total EUI: Climate Zone 3A

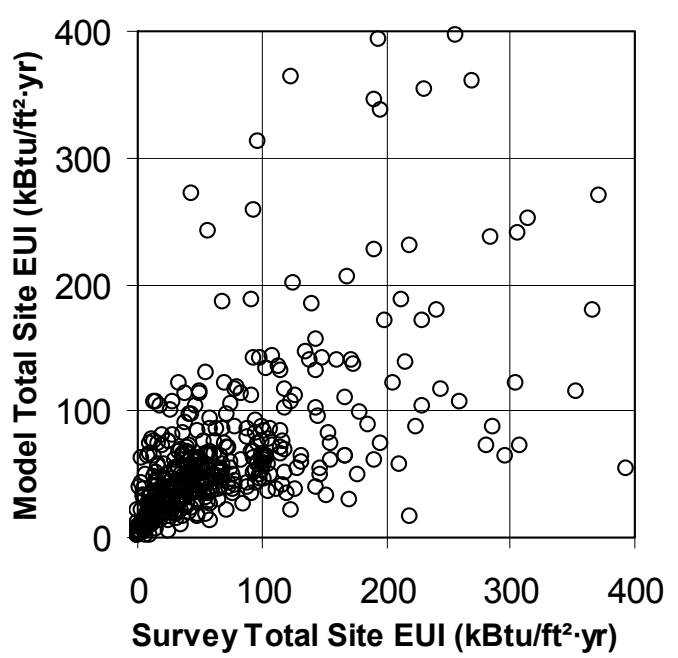

Figure A-44 Scatter Plot of Total EUI: Climate Zone 3B 


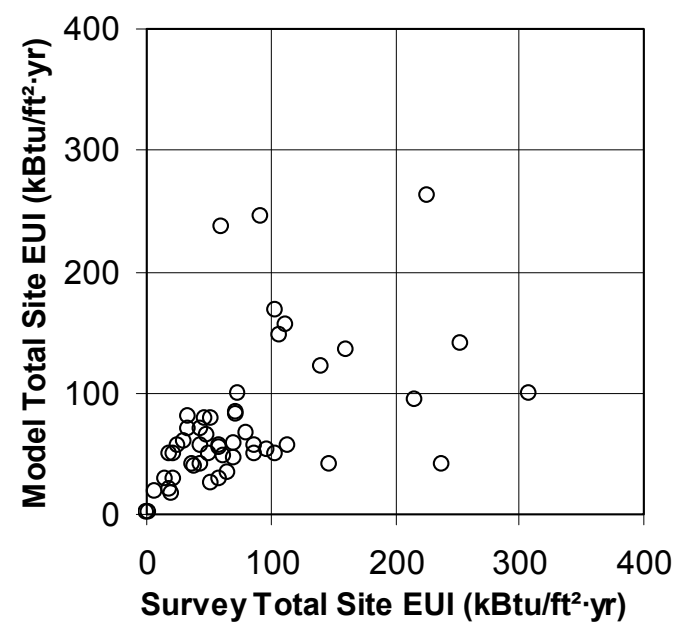

Figure A-45 Scatter Plot of Total EUI: Climate Zone 3C

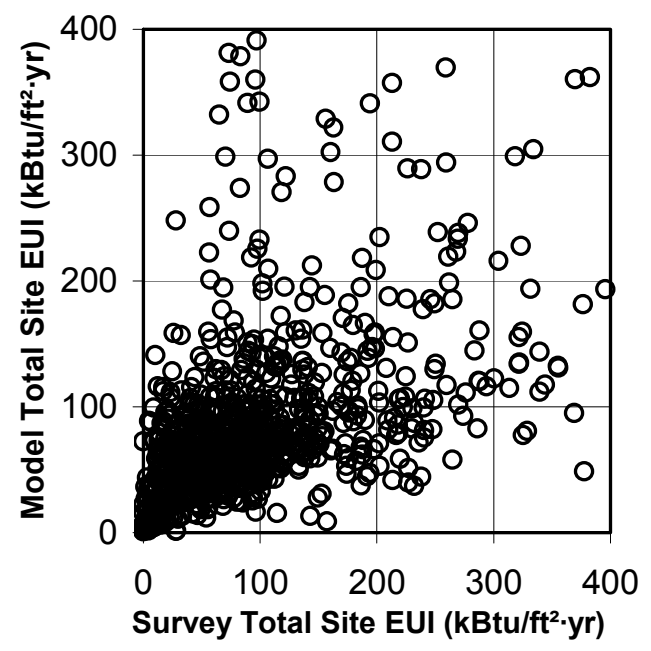

Figure A-46 Scatter Plot of Total EUI: Climate Zone 4A

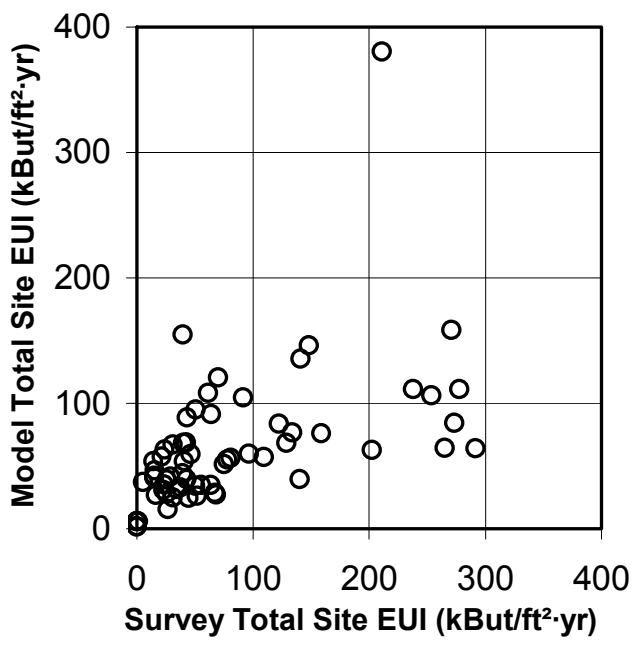

Figure A-47 Scatter Plot of Total EUI: Climate Zone 4B

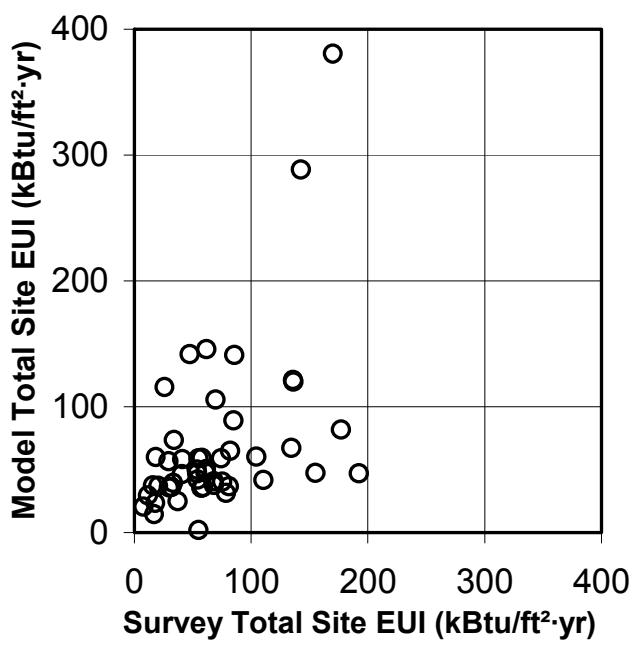

Figure A-48 Scatter Plot of Total EUI: Climate Zone 4C 


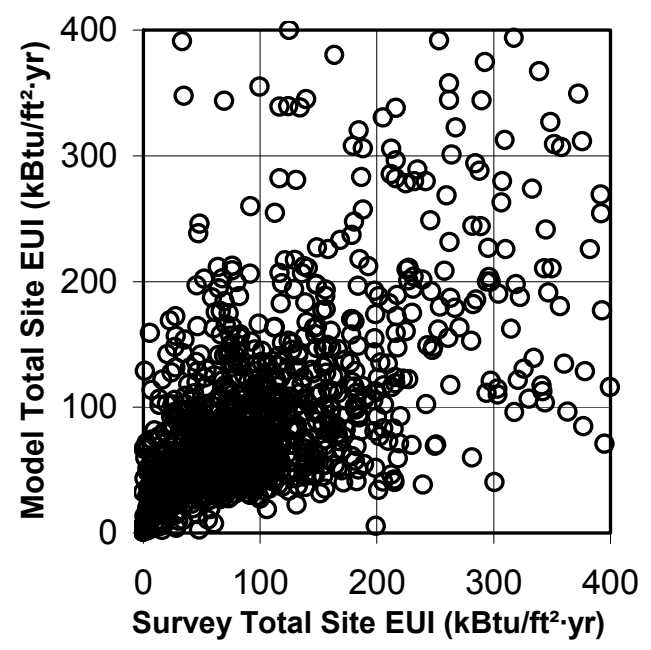

Figure A-49 Scatter Plot of Total EUI: Climate Zone 5A

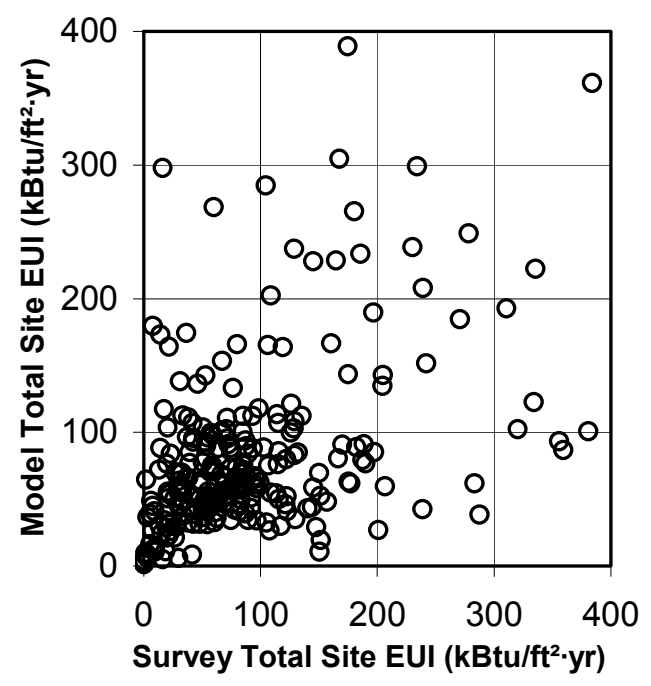

Figure A-50 Scatter Plot of Total EUI: Climate Zone 5B

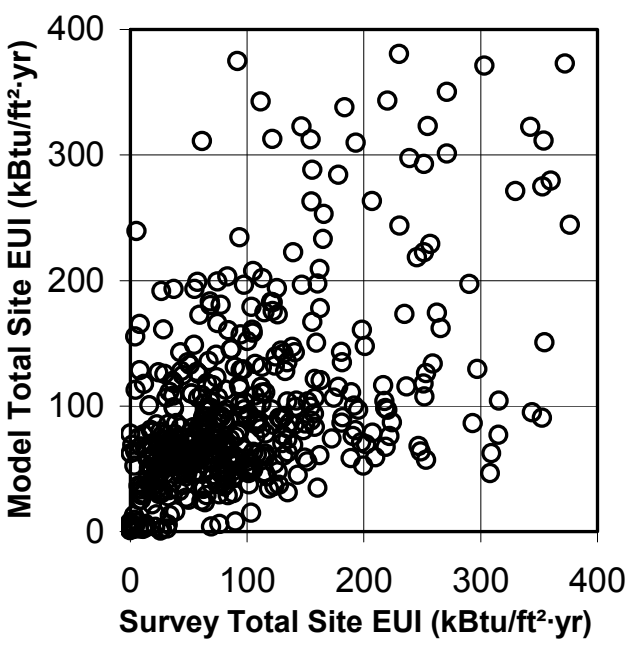

Figure A-51 Scatter Plot of Total EUI: Climate Zone 6A

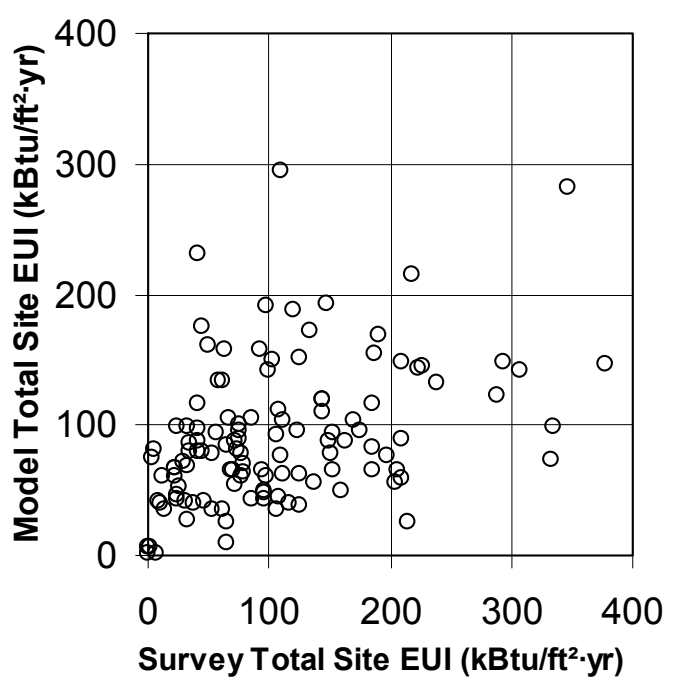

Figure A-52 Scatter Plot of Total EUI: Climate Zone 6B 


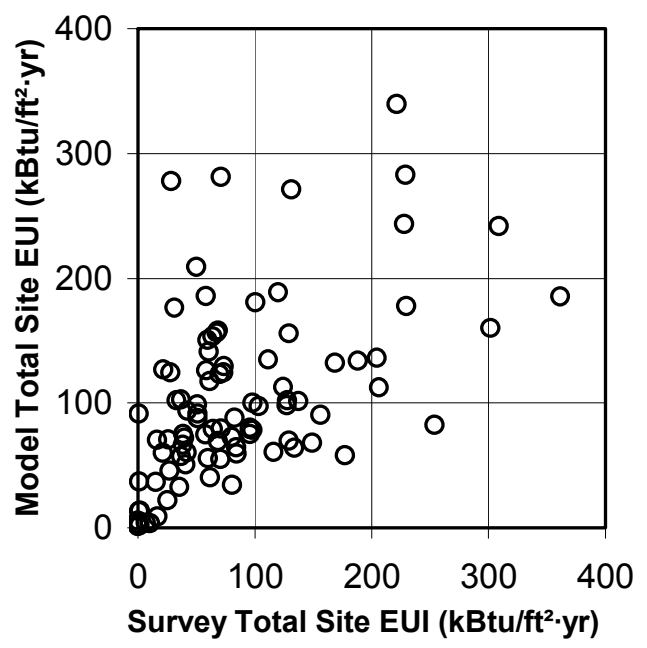

Figure A-53 Scatter Plot of Total EUI: Climate Zone 7 


\section{Appendix B. Energy Simulation Tools}

This appendix contains additional details about the computer tools NREL used for the modeling.

\section{B.1 EnergyPlus}

Modeling is all about selecting an appropriate level of detail. The spectrum of opportunity in commercial buildings is enormous, as indicated by the 75.6 billion $\mathrm{ft}^{2}\left(6.2\right.$ billion $\left.\mathrm{m}^{2}\right)$ of floor area in 2006 and the 53 billion $\mathrm{ft}^{2}\left(1.8\right.$ billion $\left.\mathrm{m}^{2}\right)$ forecast for new construction through 2030 (EIA 2007). Design has an impact on individual buildings, not on the aggregated sector. Because buildings are complex, sophisticated models must be used to conduct research. Considering that the EnergyPlus program (Crawley et al. 2001; www.energyplus.gov) is already in use, justifying new calculation routines would be difficult. EnergyPlus is a detailed, forward model that predicts energy and other performance characteristics of buildings. This section outlines some of the reasons why we selected EnergyPlus as the analysis tool.

EnergyPlus yields a high level of modeling detail, but it comes at the expense of having to prepare large and complex input files and to allow for long execution times. These issues were resolved by implementing a simple preprocessor and using distributed computing. The preprocessor automatically prepares EnergyPlus input files from a small eXtensible Markup Language (XML) file that contains input parameters that use various automatic file building routines and macro templates. One advantage of the software architecture behind EnergyPlus is that it runs well on remote machines. NREL researchers used distributed computing to run numerous simulations in parallel on multiple processors and remote machines to model some 40 buildings at a time. NREL compiled 64-bit versions of EnergyPlus version 2.0 to run on a Linux-based cluster and Windows XP x64.

Even though there are drawbacks to using a detailed program such as EnergyPlus, only such a program can fully account for the complicated interactions between climate, internal gains, building form and fabric, HVAC systems, and renewable energy systems. EnergyPlus is also a heavily tested program; formal BESTEST validation (Judkoff 1995; Neymark 2002; GARD 2004a; GARD 2004b) efforts were repeated for every release. EnergyPlus source code is well organized and available to collaborative developers. This makes customizing the program with models for building technologies straightforward.

Many simplified engineering models can predict energy performance. Some are inverse models that need to be calibrated with performance data, but such data are not available for buildings that have not yet been built or monitored. Simple models may have been formulated as steady state, or to neglect (nonintuitive) system interactions, but high-performance buildings tend to rely on system interactions and timedependent behaviors, which the simulations need to model explicitly.

EnergyPlus is a detailed energy simulation engine that requires all inputs to be explicitly defined. Although this is a large quantity of information, the definitions require that all inputs be defined, either by actual data or by defaults or assumptions. This necessitates a robust definition of the buildings, their operation, and specific operating performances of technologies.

\section{B.2 Modeling Framework and Input Parameters}

We conducted the current modeling study using an analysis framework currently called "OptEPlus" that is based on EnergyPlus. The high number of models prohibited the use of manual methods. The basic architecture of the framework is diagrammed in Figure B-1. The framework is separated into two domains: simulation management and building modeling. These domains are introduced later in this section. The interfaces between these domains are handled with text data files in XML and tabular data files produced by EnergyPlus. 


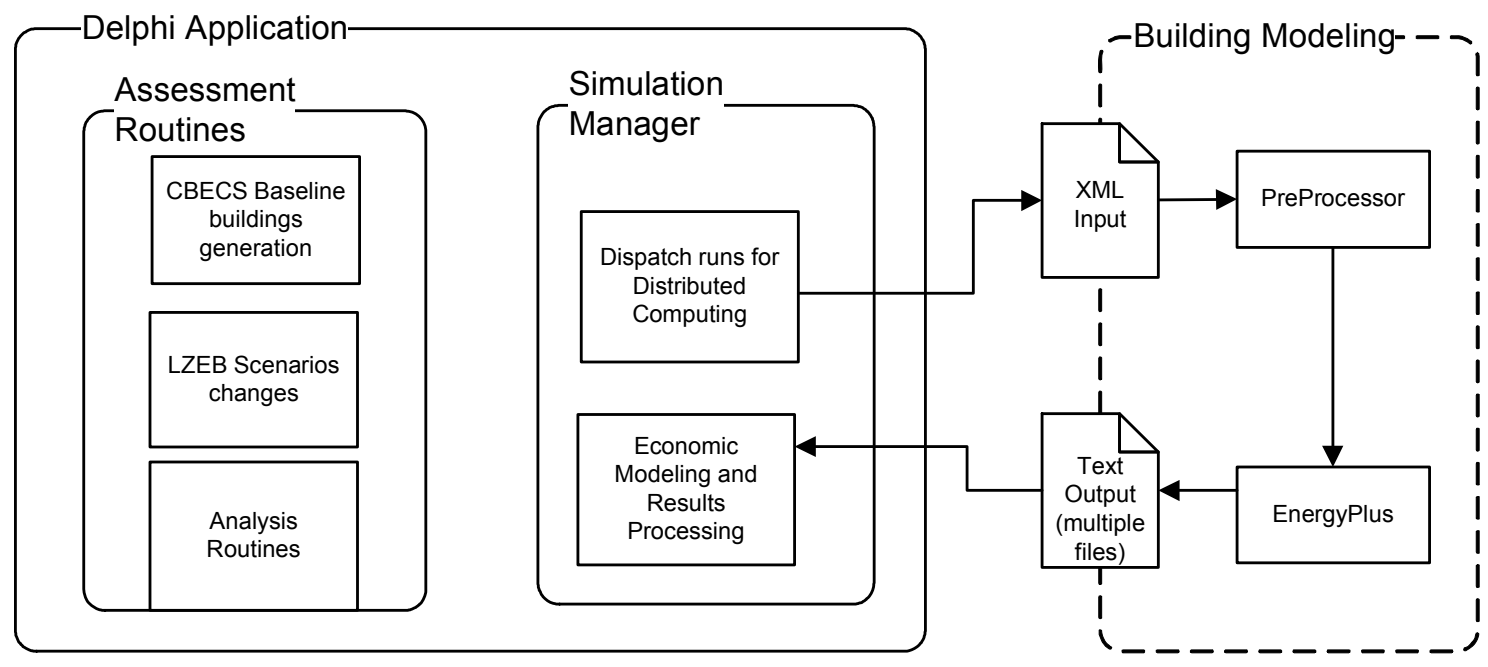

Figure B-1 Analysis framework overview

\section{B.2.1 OptEplus}

We use the term OptEPlus to refer to the entire collection of EnergyPlus input and output files, file system directories, and computer routines that are used to conduct and manage the analyses. Automated methods are needed to enable their creation, execution, and postprocessing to be managed. The simulation files and directories need structure. OptEPlus is also the name given to a computer program that collects routines and scripts that are needed to work with the simulations. The OptEPlus routines are programmed in Delphi, a powerful rapid application development environment from the Pascal family of languages. OptEPlus includes capabilities for:

- Managing and storing about 100,000 EnergyPlus simulations

- Managing the creation of XML input files

- Submitting and monitoring jobs for distributed computing on Windows machines and Linux clusters

- Postprocessing results to make them available for subsequent analyses

- Relational database for input and output

- Search routines for evaluating cost and performance of design options (single building optimization)

- CBECS Public Use Data import, filter, analysis, export, and translation to XML input files for EnergyPlus modeling

- Sector-wide scenario analysis

- Creating and running example files for EnergyPlus users via a Web site form

- Applying minimum-Standard prescriptive measures for 90.1-1999, 90.1-2004, 90.1-2007, and $189 \mathrm{P}$. 
A full set of simulation input and output files from the modeling are stored in individual directories on a 1-terabyte hard drive; a second hard drive mirrors the contents of the first for backup. EnergyPlus models that are similar to those used in this study are publicly available from the EnergyPlus Example File Generator at www.energyplus.gov. (This service is a direct by-product of the analysis routines originally implemented for the current study.)

\section{B.2.2 Building Modeling}

EnergyPlus is a detailed calculation engine described elsewhere (Crawley et al. 2001; www.energyplus.gov). The input for EnergyPlus is a detailed text file that describes the form and operation of the building. We use an in-house preprocessor to create the EnergyPlus input files. The preprocessor program takes a specially formatted (XML) text file to generate the EnergyPlus input file. This text file is an instance of a high-level data model that constitutes the interface for input to the building modeling. (This data model is cast as an XML schema called HPBxml.xsd.) We distinguish high-level parameters as those that do not, or cannot, appear directly in the simulation input file. These parameters often imply a one-to-many relationship, and imply that rules are needed to translate the parameter into multiple model input values. The main task of the preprocessor is to translate the highlevel parameters into a description of the building geometry and HVAC systems and combine that with all the other data needed to run a simulation. High-level parameters can directly represent the energy design measure separately from how that measure needs to be represented to the simulation program. An XMLbased data model was selected because XML is becoming a very popular format for text-based data. In addition, parsers are available for most programming languages, and the XML schema provides methods for validating that the input matches the expectation. The model includes a complete set of parameters that, when combined with defaults and assumptions contained in the preprocessor, can produce entire input files for EnergyPlus. Table B-1 lists the main parameters that are included in the data model. The HPBxml schema also differs from building data models (IFC or gbxml) in that it includes attributes that provide a high-level rather than a fine-grained description of all the data. The data model includes lengthy enumerations of allowable locations, weather files, materials, constructions, schedule sets, and HVAC system options that are closely coordinated with what is available in the preprocessor and input data libraries. Table B-1 also includes a column that indicates the relationship between the high-level data element and the EnergyPlus simulation file where "1-to-1" indicates a parameter that is easy to vary directly in an input file and "1-to-many" indicates a parameter that requires preprocessing. 
Table B-1 High-Level Data Model Parameters

\begin{tabular}{|c|c|c|}
\hline Element Name & Description & $\begin{array}{l}\text { Relationship to } \\
\text { E+ Input File }\end{array}$ \\
\hline WeatherFile & Name of weather file for simulation & 1-to-1 \\
\hline DesignDays & $\begin{array}{l}\text { Key for location information, including latitude, longitude, } \\
\text { elevation, design day weather, and ground temperatures }\end{array}$ & 1-to-1 \\
\hline UtilityCosts & Key for selecting energy tariff data sets & 1-to-1 \\
\hline SetsofConstructions & Key for selecting groups of materials and construction & 1-to-many \\
\hline FloorArea & Total floor area & 1-to-many \\
\hline NumFloors & Number of floors (stories) in building & 1-to-many \\
\hline AspectRatio & East-west length divided by north-south length & 1-to-many \\
\hline PerimDepth & Depth of perimeter thermal zones & 1-to-many \\
\hline FloortoFloorHeight & Distance from top of slab to top of slab on next floor & 1-to-many \\
\hline PlenumHeight & Height of suspended ceiling & 1-to-many \\
\hline Rotation & Angle of building with respect to north & 1-to-1 \\
\hline GlazingSillHeight & Distance from floor to bottom of glazing & 1-to-many \\
\hline GlazingFraction & $\begin{array}{l}\text { Ratio of total glazed area to above-grade exterior wall area } \\
\text { for an orientation }\end{array}$ & 1-to-many \\
\hline GlazingEdgeOffset & Thickness of vertical band at edge of glazings & 1-to-many \\
\hline OverhangDepth & Distance fixed shading overhang extends from wall & 1-to-many \\
\hline OverhangBase & Distance from glazing head to fixed shading overhang & 1-to-many \\
\hline DaylightingSetpoint & $\begin{array}{l}\text { Nonzero value triggers daylighting model with specified } \\
\text { lighting level }\end{array}$ & 1-to-many \\
\hline TubularDaylightDevices & $\begin{array}{l}\text { Nonzero value triggers distributing TDDs throughout core } \\
\text { zone in topmost floor at specified density }\end{array}$ & 1-to-many \\
\hline PeopleDensity & Value for design level for people per area & 1-to-many \\
\hline PlugDensity & $\begin{array}{l}\text { Value for design level for watts per unit area for plug and } \\
\text { process loads }\end{array}$ & 1-to-many \\
\hline LightIntensity & $\begin{array}{l}\text { Value for design level for watts per unit area for lighting } \\
\text { loads }\end{array}$ & 1-to-many \\
\hline InfiltrationRate & Value for design level for $\mathrm{ACH}$ & 1-to-many \\
\hline ScheduleSet & Key for selecting groups of schedules & 1-to-many \\
\hline HVACSystem & Key for selecting the type of HVAC system & 1-to-many \\
\hline OAVentPerPerson & Value for minimum outdoor air rate & 1-to-1 \\
\hline FanPressureDrop & $\begin{array}{l}\text { Value for static pressure experienced by one or more fans } \\
\text { in the HVAC system }\end{array}$ & 1-to-1 \\
\hline ChillerCOP & Value for nominal COP for mechanical cooling equipment & 1-to-1 \\
\hline GasCoilEff & Value for theoretical efficiency for gas heating equipment & 1-to-1 \\
\hline PV-Simple & Select mode for how to apply PV power systems & 1-to-many \\
\hline PVAreaFraction & $\begin{array}{l}\text { Value for fraction of available surface area that is covered } \\
\text { with PV modules }\end{array}$ & 1-to-1 \\
\hline PVEfficiency & Value for solar-to-electric conversion efficiency & 1-to-1 \\
\hline PVInverterEff & Value for DC-to-AC conversion efficiency & 1-to-1 \\
\hline MacroControl & $\begin{array}{l}\text { File system path for include files and key for selecting sets } \\
\text { of report variables }\end{array}$ & 1-to-many \\
\hline
\end{tabular}




\section{Appendix C. Input Data and Translation Rules from 2003 CBECS to Building Models}

This appendix documents how EnergyPlus input data were developed from 2003 CBECS. Fully defining a building model for the EnergyPlus simulation program requires defining a large number of detailsmany more than are available in the CBECS data. Therefore, the focus is on helping the reader understand how the detailed input data were obtained or generated to enable modeling with EnergyPlus.

We have categorized the plethora of details about the building into four groups: program, form, fabric, and equipment:

- Program refers to the architectural program, which describes how the building will be used and the services it will provide the occupants. From an energy point of view, program decisions influence many important drivers such as climate, internal gains, ventilation requirements, operating schedules, and comfort tolerances that will ultimately determine the energy performance.

- Form refers to the geometry of the building and its elements. Form has important energy implications that stem from how the building interacts with the sun and ambient conditions.

- Fabric refers the materials used to construct the building and involves decisions about insulation levels, glazing systems, and thermal mass.

- Equipment includes HVAC equipment as well as lighting systems and controls. This includes all the energy-consuming equipment that is part of the building except for plug and process load equipment selected by the occupants.

Table C-1 lists examples of various input parameters for the four categories.

Table C-1 Input Parameter Categories with Sample Parameters

\begin{tabular}{|l|l|l|l|}
\hline \multicolumn{1}{|c|}{ Program } & \multicolumn{1}{|c|}{ Form } & \multicolumn{1}{c|}{ Fabric } & \multicolumn{1}{c|}{ Equipment } \\
\hline \hline Facility location & Number of floors & Exterior walls & HVAC system types \\
Total floor area & Aspect ratio & Roof & Component efficiency \\
Schedules & Window fraction & Windows & Control settings \\
Plug and process loads & Window locations & Interior partitions & Lighting fixtures \\
Lighting quality & Shading & Internal mass & Lamp types \\
Ventilation needs & Floor height & & Daylighting controls \\
Occupancy & Orientation & & \\
Site constraints & & & \\
\hline
\end{tabular}

An obvious problem in simulating buildings from the scant data in CBECS is determining and generating geometric form. "Autobuilding" refers to the practice of automatically generating a building geometry for energy simulation. It involves developing routines that use simple rules to translate from a small set of program and form parameters to a full set of surface and zone objects that describe a building to the simulation program. The autobuilding routines used here build orthogonal, "shoebox" shaped buildings with five thermal zones per floor and bands of windows.

The first step in developing a building model is to define the architectural program for the building that is being evaluated. The remainder of this appendix focuses on the technical details for how we map various CBECS data to parameters for energy models.

The CBECS data need to be transformed into simulation files through the assignment algorithms documented in this appendix. The main elements in the data model listed in Table B-1, and the methods used to determine values for them, are revisited in the following subsections. 


\section{C.1 CBECS Variables}

The CBECS data contain information for several building types in the commercial sector. Table C-2 lists the PBAs and codes from 2003 CBECS. In this study we used the "A" data set, which does not include strip malls or enclosed malls. This is because the "B" public use data set was not yet available at the time of this study. The numbering of the PBA codes is intentionally discontinuous.

Table C-2 List of Subsectors by PBA in CBECS Data

\begin{tabular}{|c|l|}
\hline PBA Code & \multicolumn{1}{c|}{ Definition } \\
\hline \hline 1 & Vacant \\
\hline 2 & Office/professional \\
\hline 4 & Laboratory \\
\hline 5 & Nonrefrigerated warehouse \\
\hline 6 & Food sales \\
\hline 7 & Public order and safety \\
\hline 8 & Outpatient health care \\
\hline 11 & Refrigerated warehouse \\
\hline 12 & Religious worship \\
\hline 13 & Public assembly \\
\hline 14 & Education \\
\hline 15 & Food service \\
\hline 16 & Inpatient health care \\
\hline 17 & Skilled nursing \\
\hline 18 & Lodging \\
\hline 25 & Retail (except malls) \\
\hline 26 & Service \\
\hline 91 & Other \\
\hline
\end{tabular}

Table C-3 contains a list of select CBECS variables that are used to determine specific instances for input parameters during the CBECS translations. Many more CBECS variables are used in the assignment algorithms and are described in later sections of this appendix.

Table C-3 List of Important Variables in CBECS Data

\begin{tabular}{|c|l|}
\hline CBECS Variable & \multicolumn{1}{|c|}{ Definition } \\
\hline \hline CDD658 & CDDs for $65^{\circ} \mathrm{F}$ \\
\hline CENDIV8 & Census division \\
\hline HDD658 & HDDs for $65^{\circ} \mathrm{F}$ \\
\hline NFLOOR8 & Number of floors in building \\
\hline NWKER8 & Number of workers \\
\hline PBA8 & PBA \\
\hline SQFT8 & Square footage of building \\
\hline PBAPLUS8 & Extra information on building activity \\
\hline EDSEAT8 & Total seats in classrooms \\
\hline RWSEAT8 & Religious worship seating capacity \\
\hline PBSEAT8 & Public assembly seating capacity \\
\hline FDSEAT8 & Food service seating capacity \\
\hline HCBED8 & Inpatient licensed bed capacity \\
\hline RENOV8 & Renovations since 1980 \\
\hline
\end{tabular}




\section{C.2 Location and Weather File}

This assignment selects a location to assign to each building in the 2003 CBECS data set. EIA masks the actual location of the samples for anonymity, so some method of assigning a location is needed. The location of the building determines several aspects, including simulation weather file, utility tariffs, emissions factors, site-to-source conversion factor, latitude, longitude, and elevation, that are important to the modeling. CBECS masks the actual locations of sample buildings for anonymity, but does provide data for the census division (CENDIV8) and values for HDDs (HDD658) and CDDs (CDD658). Table C-4 lists the number of buildings in 2003 CBECS by census division.

Comparing 1999 CBECS to 2003 CBECS reveals significantly more spread in the variation in values for HDD and CDD across the sample for 2003 CBECS. Whether this represents an actual large increase in the real number of locations, or a different method was used to develop degree day values for reporting is unclear.

An assignment algorithm is used to select the location in the following manner. An initial set of candidate locations was assembled that was formed by a set of 232 EnergyPlus weather files that are based on TMY2 or TMY weather data locations. However, the TMY data were not used. Instead we used data from the National Solar Resource Database (NSRDB) that were reformulated into the EnergyPlus weather file format. These historical weather data files for 2003 were used in this study. HDD and CDD data (base $65^{\circ} \mathrm{F}$ ) from the 2003 weather data were also used in the location assignments. For each CBECS building, we then selected a subset of these candidate locations that lie within the appropriate census division. (Table C-4 lists the number of candidate locations by census division.) This subset of candidate locations is subsequently searched to find the location that most closely matches the reported HDDs and CDDs. A "brute force" search algorithm was used, where for each possible assignment we calculated the root mean square of the combined deviations for HDDs and CDDs. The resulting array of root mean square error values was then searched for the minimum and the location with the lowest error selected for the assignment.

Table C-4 Summary of Location Assignments for All Buildings in 2003 CBECS

\begin{tabular}{|l|c|c|c|}
\hline Census Division & $\begin{array}{c}\text { Number of } \\
\text { Buildings in } \\
\text { 2003 CBECS }\end{array}$ & $\begin{array}{c}\text { Number of } \\
\text { Candidate Weather } \\
\text { Data Locations }\end{array}$ & $\begin{array}{c}\text { Number of } \\
\text { Locations Assigned }\end{array}$ \\
\hline 1 New England & 195 & 9 & 8 \\
\hline 2 Middle Atlantic & 641 & 18 & 15 \\
\hline 3 East North Central & 860 & 30 & 29 \\
\hline 4 West North Central & 452 & 30 & 27 \\
\hline 5 South Atlantic & 912 & 32 & 28 \\
\hline 6 East South Central & 279 & 14 & 13 \\
\hline 7 West South Central & 579 & 26 & 23 \\
\hline 8 Mountain & 305 & 41 & 20 \\
\hline 9 Pacific & 597 & 32 & 19 \\
\hline All & 4,820 & 232 & 181 \\
\hline
\end{tabular}

The results of applying the location assignment algorithm are summarized in Table C-4. Figure C-1 and Figure C-2 compare the HDD and CDD values from the assignments and show the level of agreement between the locations that were selected for modeling and the data that were available from the 2003 CBECS (based on these weather criteria). The selection algorithm chose 181 unique locations, which represented $78 \%$ of the candidate locations with weather data. 


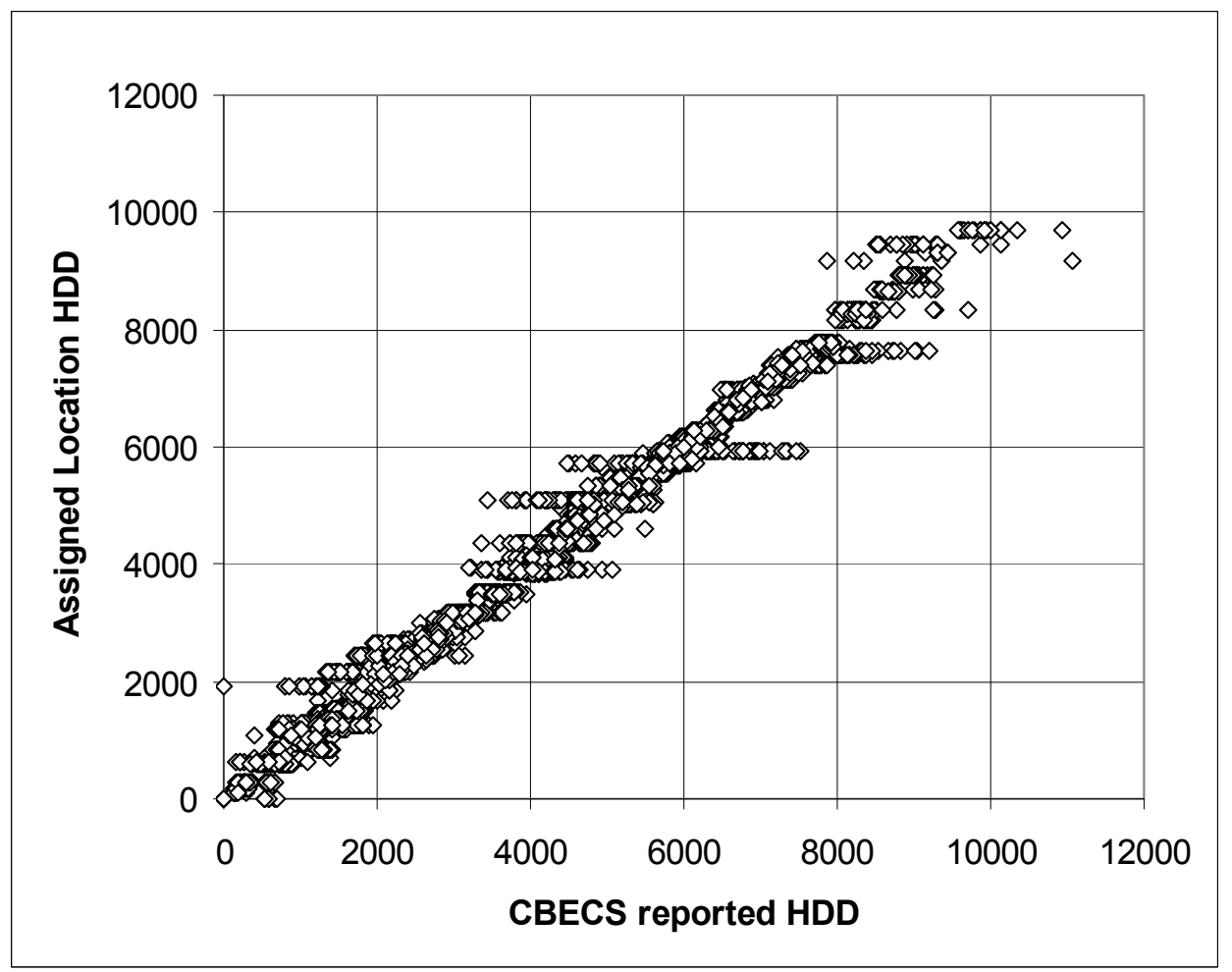

Figure C-1 Comparison of HDDs between assigned locations and 2003 CBECS

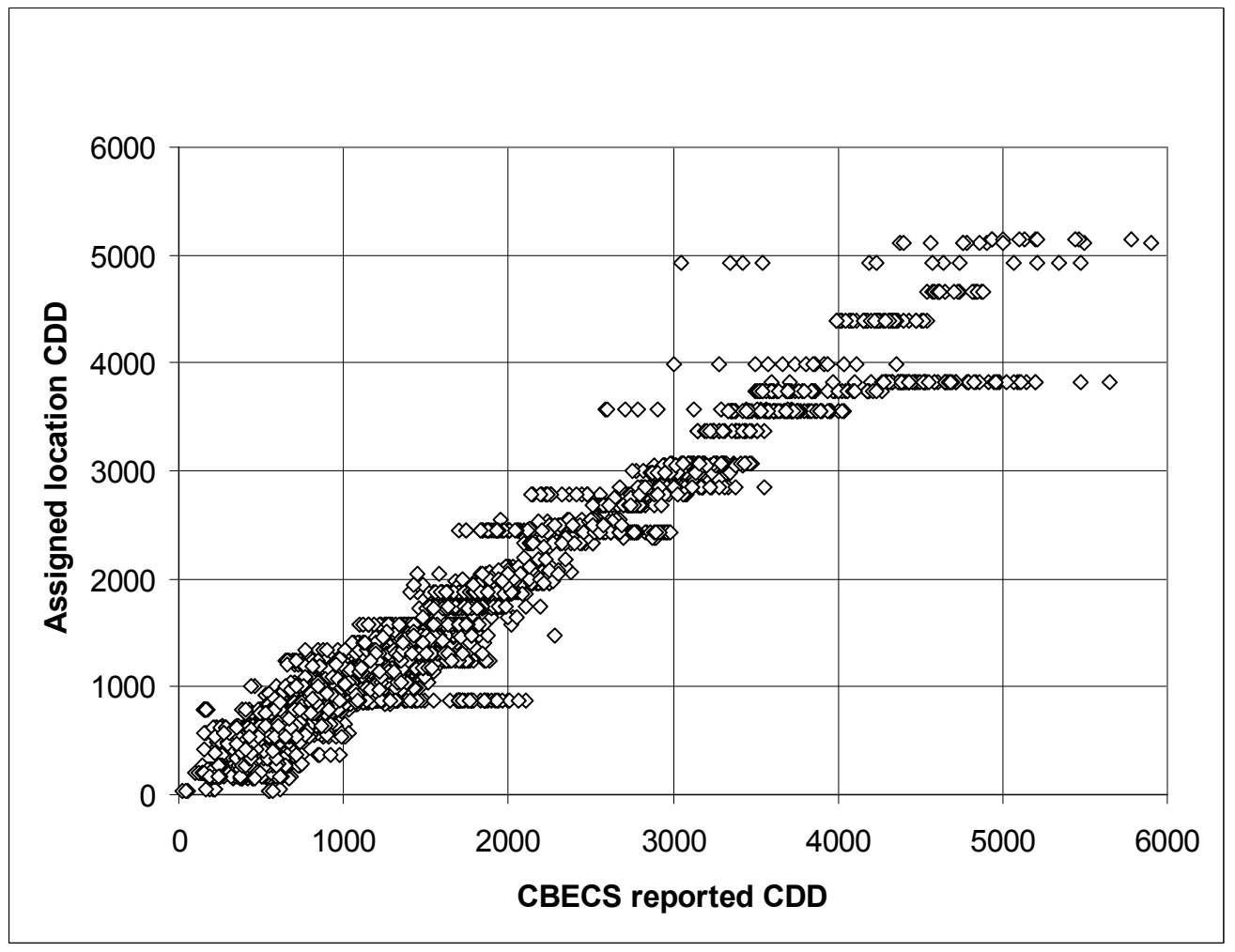

Figure C-2 Comparison of CDDs between assigned locations and 2003 CBECS 


\section{C.3 Utility Costs}

Utility rate schedules, or tariffs, are needed to calculate energy costs. This section describes how rates are assigned to each building. As discussed in Section C.2, the building location is assigned with census division and degree-day data. This location is then used to determine utility tariffs for the building. Electricity and natural gas are the only two types of energy with costs modeled in the study. The methods for selecting tariffs are described in the following two subsections.

\section{C.3.1 Electricity}

The models include the energy and demand charges and geographic variations. In many commercial buildings, demand charges can account for $50 \%$ of the total electricity bill. As buildings strive for lower energy, anecdotal experience shows that the demand savings are lower than the energy savings and become a larger portion of the bill. Using virtual or average rates does not account for this and can inflate the appeal of technologies such as PV power systems, solar hot water systems, and daylighting. Other techniques, such as increasing thermal envelope, overhangs, and glazing selection can realize demand savings if the HVAC systems are sized appropriately. Technologies such as improved lighting efficiency can affect both demand and energy. To fully realize the savings and interactions, demand and energy charges must be computed.

Tariffs for electricity pricing are complex. Averaged pricing data are readily available from EIA, but do not provide the necessary detail for calculating realistic rate structures. For the study, a set of realistic rate structures was developed in three steps:

- Determine specific utility companies for each location.

- Access and understand the electricity rate schedules published by those utilities.

- Translate those rates into the input form needed for calculating the energy costs in EnergyPlus.

Table C-5 lists the utility companies used in the study. (Documenting the actual tariffs would be complex and lengthy, so the details of each are left to the EnergyPlus input data.) Forty utility companies were identified through the following process:

1. For each location assignment, we identified the largest utilities in the state from EIA data. These data show the total annual sales to commercial customers for the five largest utilities (EIA State Electricity Profiles for 2002 [www.eia.doe.gov/cneaf/electricity/st_profiles/e_profiles_sum.html]; accessed January 13, 2005).

2. We examined the tariffs (available from a Web-based central repository run by the Tariff Analysis Project (TAP) [http://tariffs.lbl.gov/; accessed February 2005]) to determine whether data for one of the largest utilities were available. Largest utilities were determined from EIA State Electricity Profiles for 2002

(www.eia.doe.gov/cneaf/electricity/st_profiles/e_profiles_sum.html; accessed January 13, 2005).

3. If TAP did not have data for a large and dominant utility, we went directly to the electric utility company Web sites to verify that the tariff data were available. Additional resolution in rates is required for different sizes of utility customers with up to five tariffs for each location, depending on how each utility organizes its schedules.

4. Tax rates on energy costs were assumed to correlate with state sales tax using state sales tax plus $2 \%$. State tax rates were developed from http://thestc.com/Strates.stm (accessed January 19, 2005).

5. We used the tariff calculations from EnergyPlus to apply the lowest cost tariff when more than one rate structure might qualify. The total number of electricity tariffs is 110 for 40 utilities.

6. We selected the assignments by choosing the largest utilities that serve commercial customers based on the EIA data and what was available in TAP. New York and California were divided 
into two regions. The electrical rate modeling is all based on secondary power (no customer ownership of transformer).

Table C-5 Electric Utility Company Tariff Summary

\begin{tabular}{|c|c|c|c|}
\hline Electric Utility Company & $\begin{array}{c}\text { Utility Tax Rate } \\
\text { (\%) }\end{array}$ & $\begin{array}{c}\text { Number of } \\
\text { Electricity Rates }\end{array}$ & $\begin{array}{l}\text { Effective Date } \\
\quad \text { for Tariff }\end{array}$ \\
\hline Connecticut Light \& Power & 8 & 4 & $\begin{array}{l}2004 / 06 / 23 \\
2005 / 01 / 01 \\
\end{array}$ \\
\hline Energy Atlantic LLC & 7 & 3 & $2004 / 07 / 01$ \\
\hline Boston Edison Co. & 9 & 5 & 2004/01/01 \\
\hline Consolidated Edison Co.-NY & 8 & 2 & $2004 / 11 / 01$ \\
\hline Niagara Mohawk Power Co. & 8 & 3 & $2005 / 01 / 05$ \\
\hline PECO Energy Co. & 8 & 1 & $2004 / 11 / 01$ \\
\hline Public Service Electric \& Gas Co. & 8 & 3 & $2004 / 06 / 01$ \\
\hline PSI Energy, Inc. & 8 & 2 & $2004 / 05 / 24$ \\
\hline Ohio Edison Co. & 8 & 1 & $2003 / 01 / 23$ \\
\hline Wisconsin Public Service Corp. & 7 & 4 & $2004 / 01 / 01$ \\
\hline Consumers Energy Co. & 8 & 2 & $2005 / 01 / 03$ \\
\hline Union Electric Co. & 6.25 & 2 & $2004 / 04 / 01$ \\
\hline MidAmerican Energy Co. & 7 & 2 & $2003 / 01 / 01$ \\
\hline Northern States Power Co. & 6 & 2 & $2003 / 05 / 26$ \\
\hline Lincoln Electric System & 7.5 & 3 & $2004 / 10 / 15$ \\
\hline Florida Power \& Light Co. & 8 & 3 & $2005 / 01 / 04$ \\
\hline Georgia Power Co. & 6 & 4 & $2002 / 03 / 01$ \\
\hline Virginia Electric \& Power Co. & 6 & 3 & $2004 / 01 / 01$ \\
\hline Duke Energy Corp. & 9 & 1 & $2004 / 07 / 01$ \\
\hline Allegheny Power Co. & 8 & 3 & $2000 / 07 / 01$ \\
\hline Delmarva Power \& Light Co. & 2 & 3 & $2002 / 10 / 01$ \\
\hline Alabama Power Co. & 6 & 3 & $2004 / 07 / 01$ \\
\hline Entergy Mississippi, Inc. & 7 & 2 & $2002 / 12 / 31$ \\
\hline City of Memphis & 9 & 3 & $2003 / 12 / 30$ \\
\hline Reliant Energy HL\&P & 8.25 & 2 & $2004 / 12 / 21$ \\
\hline Entergy Louisiana & 6 & 5 & $2003 / 01 / 01$ \\
\hline Entergy Arkansas & 10 & 2 & $2004 / 01 / 01$ \\
\hline Arizona Public Service Co. & 7.6 & 1 & $2003 / 07 / 01$ \\
\hline Public Service Co. of N.M. & 7 & 3 & $2003 / 09 / 01$ \\
\hline Idaho Power Co. & 8 & 3 & $2004 / 07 / 28$ \\
\hline Nevada Power Co. & 6.5 & 4 & $2004 / 06 / 01$ \\
\hline Public Service Co. of Colorado & 4.9 & 2 & $2004 / 04 / 02$ \\
\hline NorthWestern Energy & 2 & 2 & $2005 / 02 / 01$ \\
\hline Hawaiian Electric Co. Inc. & 6 & 3 & $2003 / 01 / 01$ \\
\hline Southern Cal Edison & 8 & 3 & $2004 / 07 / 16$ \\
\hline Pacific Gas \& Electric & 8 & 3 & $2004 / 12 / 01$ \\
\hline PacifiCorp & 2 & 8 & $2004 / 01 / 01$ \\
\hline Puget Sound Energy & 8.5 & 3 & $2004 / 07 / 01$ \\
\hline Chugach Electric Association, Inc. & 2 & 2 & $2004 / 10 / 01$ \\
\hline
\end{tabular}

Utility customers also pay taxes. Tax rates on utilities vary at the city and county government levels, so developing a broad set of specific tax rates is difficult. TAP neglected taxes. Most utility companies do not publish tax rates with their tariffs because they vary within the service territory. Collecting sufficient data about tax rates to formulate appropriate averages for use in the study would require a major research effort. However, taxes form an important part of energy costs in the commercial sector, and therefore need to be included in the modeling. These data were filled by assuming that energy taxes are equal to 
the state sales tax rate plus $2 \%$. For example, if a state has a $5 \%$ sales tax, we model the utility tax rate as $7 \%$. All the locations in a state are assigned the same tax rate. Table C-5 also lists these tax rate assignments for each location in the study. Although many institutions do not have to pay taxes, the current modeling does not distinguish between the owners of the buildings, so all are assumed to pay taxes.

Many commercial buildings have multiple owners or tenants, so there are many more utility customers than buildings in the United States. In some cases, a customer has multiple buildings in a single utility district. However, we could not identify good methods or supporting data to handle these real-world complexities, and assumed that each building has only one electricity customer. This will tend to make for fewer and larger electrical customers and can be expected to affect our modeling results by decreasing the significance of monthly service charges and shifting tariff schedules upward to those of larger customers (which tends to increase demand charges relative to energy charges).

\section{C.3.2 Natural Gas}

Table C-6 lists the averages of the monthly natural gas rates that are assigned to the models. The actual values are monthly and were taken from the last 12 months of recent EIA data at the state level. The gas tariff data set uses the same locations and tax rates as the electricity. Most natural gas rates are either fixed price or have some limited price tiers. Very few rates are based on demand charges in the same way electricity is. Metering and billing charges can be significant; however, with an analysis that looks at differences, these charges are not important when cost savings are calculated. Also, our understanding is that these EIA data are for revenues and already include monthly service charges. Retail gas companies tend to have small service areas; many small companies provide natural gas.

Table C-6 Utility Rate Assignments for Natural Gas Average Retail Prices for Commercial Customers Assigned to Electric Utility Territory*

\begin{tabular}{|l|c|c|}
\hline \multicolumn{1}{|c|}{$\begin{array}{c}\text { Electric Utility Company } \\
\text { Assignment }\end{array}$} & $\begin{array}{c}\text { Utility Tax Rate } \\
\text { (\%) }\end{array}$ & $\begin{array}{c}\text { Average Annual Gas Rate } \\
\text { (\$/MCF) }\end{array}$ \\
\hline \hline Connecticut Light \& Power & 8 & 10.94 \\
\hline Energy Atlantic LLC & 7 & 11.53 \\
\hline Boston Edison Co. & 9 & 11.20 \\
\hline Consolidated Edison Co.-NY & 8 & 9.22 \\
\hline Niagara Mohawk Power Co. & 8 & 9.22 \\
\hline PECO Energy Co. & 8 & 10.57 \\
\hline Public Service Electric \& Gas Co. & 8 & 9.99 \\
\hline PSI Energy, Inc. & 8 & 8.88 \\
\hline Ohio Edison Co. & 8 & 8.89 \\
\hline Wisconsin Public Service Corp. & 7 & 8.40 \\
\hline Consumers Energy Co. & 8 & 8.31 \\
\hline Union Electric Co. & 6.25 & 10.19 \\
\hline MidAmerican Energy Co. & 7 & 8.98 \\
\hline Northern States Power Co. & 6 & 7.98 \\
\hline Lincoln Electric System & 7.5 & 7.31 \\
\hline Florida Power \& Light Co. & 8 & 11.21 \\
\hline Georgia Power Co. & 6 & 10.98 \\
\hline Virginia Electric \& Power Co. & 6 & 10.15 \\
\hline Duke Energy Corp. & 9 & 10.26 \\
\hline Allegheny Power Co. & 8 & 10.34 \\
\hline Delmarva Power \& Light Co. & 2 & 10.15 \\
\hline Alabama Power Co. & 6 & 11.15 \\
\hline Entergy Mississippi, Inc. & 7 & 7.99 \\
\hline City of Memphis & 9 & 9.20 \\
\hline Reliant Energy HL\&P & 8.25 & 8.01 \\
\hline
\end{tabular}




\begin{tabular}{|l|c|c|}
\hline \multicolumn{1}{|c|}{$\begin{array}{c}\text { Electric Utility Company } \\
\text { Assignment }\end{array}$} & $\begin{array}{c}\text { Utility Tax Rate } \\
\text { (\%) }\end{array}$ & $\begin{array}{c}\text { Average Annual Gas Rate } \\
\text { (\$/MCF) }\end{array}$ \\
\hline \hline Entergy Louisiana & 6 & 9.43 \\
\hline Entergy Arkansas & 10 & 9.18 \\
\hline Arizona Public Service Co. & 7.6 & 8.47 \\
\hline Public Service Co. of N.M. & 7 & 7.75 \\
\hline Idaho Power Co. & 8 & 8.39 \\
\hline Nevada Power Co. & 6.5 & 8.17 \\
\hline Public Service Co. of Colorado & 4.9 & 7.31 \\
\hline NorthWestern Energy & 2 & 9.28 \\
\hline Hawaiian Electric Co. Inc. & 6 & 20.74 \\
\hline Southern Cal Edison & 8 & 8.19 \\
\hline Pacific Gas \& Electric & 8 & 8.19 \\
\hline PacifiCorp & 2 & 8.70 \\
\hline Puget Sound Energy & 8.5 & 8.04 \\
\hline Chugach Electric Association, Inc. & 2 & 4.38 \\
\hline
\end{tabular}

*Source: Energy Information Agency:

http://tonto.eia.doe.gov/dnav/ng/ng pri sum a EPGO PCS DMcf m.htm (accessed January 12, 2005)

\section{C.4 Fuel Factors for Electricity to Source Energy}

The factors used to convert from site energy use to source energy are listed in Table C-7. These data were compiled for 2004 by Deru and Torcellini (2007). This data set was selected because it provides the latest data for source energy factors that are specially tailored for building energy analyses and offers a regional breakdown by state. These factors are the "total with precombustion" from Table B-9 in Deru and Torcellini (2007) and include the source energy consequences of nuclear generation. Source energy results are not included in this report, but are available for subsequent studies. 
Table C-7 Site-to-Source Conversion Factors for Electricity

\begin{tabular}{|c|c|c|c|}
\hline Census Division & State & $\begin{array}{l}\text { Electric Utility Company } \\
\text { Assignment }\end{array}$ & $\begin{array}{l}\text { Site-to-Source Factor for } \\
\text { Electricity }\end{array}$ \\
\hline \multirow{4}{*}{ New England } & CT & Connecticut Light \& Power & 3.310 \\
\hline & $\mathrm{ME}$ & Energy Atlantic LLC & 2.904 \\
\hline & $\mathrm{RI}$ & Connecticut Light \& Power & 2.779 \\
\hline & MA & Boston Edison Co. & 3.309 \\
\hline \multirow{5}{*}{ Middle Atlantic } & NY & Consolidated Edison Co.-NY & 3.229 \\
\hline & NY & Niagara Mohawk Power Co. & 3.229 \\
\hline & NY & Niagara Mohawk Power Co. & 3.229 \\
\hline & PA & PECO Energy Co. & 3.453 \\
\hline & $\mathrm{NJ}$ & Public Service Electric \& Gas Co. & 3.500 \\
\hline \multirow{4}{*}{ East North Central } & IN & PSI Energy, Inc & 3.611 \\
\hline & $\mathrm{OH}$ & Ohio Edison Co. & 3.448 \\
\hline & WI & Wisconsin Public Service Corp. & 3.635 \\
\hline & $\mathrm{MI}$ & Consumers Energy Co. & 3.485 \\
\hline \multirow{4}{*}{ West North Central } & $\mathrm{MO}$ & Union Electric Co. & 3.551 \\
\hline & $\mathrm{IA}$ & MidAmerican Energy Co. & 3.694 \\
\hline & SD & Northern States Power Co. & 2.484 \\
\hline & $\mathrm{NE}$ & Lincoln Electric System & 3.524 \\
\hline \multirow{6}{*}{ South Atlantic } & $\mathrm{FL}$ & Florida Power \& Light Co. & 3.374 \\
\hline & GA & Georgia Power Co. & 3.397 \\
\hline & VA & Virginia Electric \& Power Co. & 3.613 \\
\hline & $\mathrm{NC}$ & Duke Energy Corp. & 3.307 \\
\hline & WV & Allegheny Power Co. & 3.391 \\
\hline & $\mathrm{DE}$ & Delmarva Power \& Light Co. & 3.941 \\
\hline \multirow{3}{*}{ East South Central } & $\mathrm{AL}$ & Alabama Power Co. & 3.332 \\
\hline & MS & Entergy Mississippi, Inc. & 3.540 \\
\hline & $\mathrm{TN}$ & City of Memphis & 3.194 \\
\hline \multirow{3}{*}{ West South Central } & TX & Reliant Energy HL\&P & 3.712 \\
\hline & LA & Entergy Louisiana & 3.411 \\
\hline & AR & Entergy Arkansas & 3.264 \\
\hline \multirow{6}{*}{ Mountain } & $A Z$ & Arizona Public Service Co. & 3.202 \\
\hline & NM & Public Service Co. of N.M. & 3.620 \\
\hline & ID & Idaho Power Co. & 1.597 \\
\hline & NV & Nevada Power Co. & 3.327 \\
\hline & $\mathrm{CO}$ & Public Service Co. of Colorado & 3.380 \\
\hline & MT & NorthWestern Energy & 3.009 \\
\hline \multirow{6}{*}{ Pacific } & $\mathrm{HI}$ & Hawaiian Electric Co. Inc. & 4.022 \\
\hline & $\mathrm{CA}$ & Southern Cal Edison & 3.135 \\
\hline & $\mathrm{CA}$ & Pacific Gas \& Electric & 3.135 \\
\hline & OR & PacifiCorp & 1.690 \\
\hline & WA & Puget Sound Energy & 1.753 \\
\hline & AK & Chugach Electric Association, Inc. & 3.650 \\
\hline
\end{tabular}

Source: Deru and Torcellini 2007 (Table B-9) 


\section{C.5 Schedules}

This section documents how various CBECS variables were used to generate a unique set of input schedules for each building. In EnergyPlus, schedules are important model inputs that describe timedependent operation of building systems and occupant behaviors. Table C-8 lists schedules that were created for the modeling. 
Table C-8 Schedules Generated for Each Building

\begin{tabular}{|c|c|c|c|c|}
\hline Schedule Purpose & Description & Type (Units) & Name in Input File & Notes \\
\hline HVAC operation & $\begin{array}{l}\text { HVAC operation - is } \\
\text { HVAC available }\end{array}$ & Fraction & HVACOperationSchd & \\
\hline $\begin{array}{l}\text { Lighting system } \\
\text { operation }\end{array}$ & $\begin{array}{l}\text { Lighting - Same for all } \\
\text { zones }\end{array}$ & Fraction & BLDG_LIGHT_SCH & \\
\hline $\begin{array}{l}\text { Plug and process } \\
\text { loads }\end{array}$ & $\begin{array}{l}\text { Equipment - Same for all } \\
\text { zones }\end{array}$ & Fraction & BLDG_EQUIP_SCH & \\
\hline People & $\begin{array}{l}\text { Occupancy - Same for all } \\
\text { zones }\end{array}$ & Fraction & BLDG_OCC_SCH & \\
\hline Hot water use & Service Hot Water & Fraction & BLDG_SWH_SCH & \\
\hline People activity & Activity level schedule & $\begin{array}{l}\text { Number } \\
\text { (W/Person) }\end{array}$ & ACTIVITY_SCH & \begin{tabular}{|l|l|} 
Pg 283 \\
IORef
\end{tabular} \\
\hline People activity & $\begin{array}{l}\text { Work efficiency, } 0 \\
\text { signifies that all energy is } \\
\text { heat gain }\end{array}$ & Fraction & WORK_EFF_SCH & Always 0 \\
\hline People clothing & Clothing schedule & Number (Clo) & CLOTHING_SCH & \\
\hline Comfort air velocity & Amount of air movement & Number $(\mathrm{m} / \mathrm{s})$ & AIR_VELO_SCH & Always 0.2 \\
\hline Infiltration & $\begin{array}{l}\text { Infiltration - inverse of } \\
\text { HVAC operation } \\
\text { schedule }\end{array}$ & On/Off & INFIL_SCH & \\
\hline Plant availability & $\begin{array}{l}\text { Plant availability schedule } \\
\text { - for VAV systems }\end{array}$ & On/Off & PlantOnSched & Always 1 \\
\hline Fan availability & $\begin{array}{l}\text { Fan availability schedule } \\
\text { - for VAV systems }\end{array}$ & On/Off & FAN_SCH & Always 1 \\
\hline Reheat availability & $\begin{array}{l}\text { Reheat coil availability - } \\
\text { for VAV systems }\end{array}$ & On/Off & ReheatCoilAvailSched & Always 1 \\
\hline Cooling availability & $\begin{array}{l}\text { Cooling coil availability - } \\
\text { for VAV systems }\end{array}$ & On/Off & CoolingCoilAvailSched & Always 1 \\
\hline Thermostat & Heating set point & Number $\left({ }^{\circ} \mathrm{C}\right)$ & HTGSETP_SCH & \\
\hline Thermostat & Cooling set point & Number $\left({ }^{\circ} \mathrm{C}\right)$ & CLGSETP_SCH & \\
\hline Humidistat & Humidity Setpoint & Number $(\mathrm{RH})$ & $\begin{array}{l}\text { Humidity Setpoint } \\
\text { Schedule }\end{array}$ & Always 50 \\
\hline $\begin{array}{l}\text { Ventilation } \\
\text { requirements }\end{array}$ & Minimum outside air & On/Off & MinOA_Sched & $\begin{array}{l}\text { Outside Air } \\
\text { Controller }\end{array}$ \\
\hline Thermostat & $\begin{array}{l}\text { Type of zone control }(4= \\
\text { dual set point) }\end{array}$ & Number (0-4) & \begin{tabular}{|l|} 
Dual Zone Control \\
Type Sched \\
\end{tabular} & Always 4 \\
\hline $\begin{array}{l}\text { Air system cold } \\
\text { deck temperature }\end{array}$ & $\begin{array}{l}\text { Schedule to determine } \\
\text { set points }\end{array}$ & Number $\left({ }^{\circ} \mathrm{C}\right)$ & $\begin{array}{l}\text { Seasonal-Reset- } \\
\text { Supply-Air-Temp-Sch }\end{array}$ & \\
\hline $\begin{array}{l}\text { Chilled water } \\
\text { supply temperature }\end{array}$ & $\begin{array}{l}\text { Cooling water } \\
\text { temperature }\end{array}$ & Number $\left({ }^{\circ} \mathrm{C}\right)$ & $\begin{array}{l}\text { CW-Loop-Temp- } \\
\text { Schedule } \\
\end{array}$ & Always 6.7 \\
\hline $\begin{array}{l}\text { Hot water supply } \\
\text { temperature }\end{array}$ & Hot water temperature & Number $\left({ }^{\circ} \mathrm{C}\right)$ & $\begin{array}{l}\text { HW-Loop-Temp- } \\
\text { Schedule } \\
\end{array}$ & Always 60 \\
\hline $\begin{array}{l}\text { Air system hot deck } \\
\text { temperature }\end{array}$ & $\begin{array}{l}\text { Heating supply air } \\
\text { temperature }\end{array}$ & Number $\left({ }^{\circ} \mathrm{C}\right)$ & $\begin{array}{l}\text { Heating-Supply-Air- } \\
\text { Temp-Sch }\end{array}$ & \\
\hline
\end{tabular}

For many schedules, the building's hours of operation drive the underlying time dependence. Therefore, the first step for each building is to apply the CBECS variables listed in Table C-9 to model the hours of operation. 
Table C-9 CBECS Variables Used To Determine Hours of Operation

\begin{tabular}{|c|l|l|}
\hline CBECS Variable & \multicolumn{1}{|c|}{ Description } & \multicolumn{1}{|c|}{ Notes } \\
\hline \hline OPEN24 & $\begin{array}{l}\text { Open 24 hours } \\
1-\text { Yes } \\
2-\text { No }\end{array}$ & \\
\hline \multirow{3}{*}{ DAYSOPN } & $\begin{array}{l}\text { Days open (Mon-Fri) } \\
1-\text { Open all five days } \\
2-\text { Open some of these days } \\
3-\text { Not open at all }\end{array}$ & \\
& $\begin{array}{l}\text { Open on weekends } \\
1-\text { Yes } \\
2-\text { No }\end{array}$ & \\
OPENWE & $\begin{array}{l}\text { Total weekly operating hours } \\
\text { XX - Value }\end{array}$ & \\
\hline WKHRS8 & More specific building activity & $\begin{array}{l}\text { If } \geq 997 \text { then unknown (DNE } \\
\text { in dataset) }\end{array}$ \\
\hline PBAPLUS & $\begin{array}{l}\text { Month ready for occupancy in 2003 } \\
0-\text { N/A } \\
1: 12-\text { Month }\end{array}$ & \\
\hline MONCON & $\begin{array}{l}\text { Space vacant 3 consecutive months } \\
1-\text { Yes } \\
2-\text { No }\end{array}$ & $\begin{array}{l}\text { If } 0 \text { then not used } \\
\text { (variable not used because } \\
\text { of inconsistencies) }\end{array}$ \\
\hline PORVAC & Months in use past 12 months & \\
\hline MONUSE & &
\end{tabular}

The variables defined in Table C-9 were used to determine the typical operating schedules of the building. If the building was defined to be open 24 hours, the schedules were set to always open. If the building was not open 24 hours, other logic had to be used. The idea was to generate a typical week by applying the CBECS variables. First, if the building was open only on the weekends, we had to determine if it was open on both Saturday and Sunday, or just one of the days. If the total weekly hours were more than 20 (assumed value), the building was open both Saturday and Sunday. However, if it was open 20 hours or fewer, the building was open only one day. If the building was open both days of the weekend, another assumed variable weighted either Saturday or Sunday, based on the more specific building activities. These are defined in Table C-10.

If the building was open during the week and during the weekend, another set of logic was needed to interpolate the hours of operation. First, the favored day of the weekend was assigned a couple of hours (to be targeted later to match the total weekly hours). The hours were assigned to the middle of the day, again defined in Table C-10. If the total weekly hours were fewer than 10 (assumed value), the middle of the day may be different because of the limited hours. Finally, if the total weekly hours were more than 70 (assumed value), the building was assumed to be open the other day of the weekend.

The next step was to assign the correct number of hours to the week by first assigning the average daily hours to the opened days. Sometimes a building was open only some weekdays. In this case the days open were randomly chosen based on the number of days needed to be open 10 hours per day.

Sometimes the total number of hours open could not be distributed evenly across the days open. In this case, the missing hours were randomly assigned to the days. First a random day was chosen, and yet another random process determined whether to assign the hour to the beginning of the day or to the end of the day. If the building was open during the weekend, the weekends were weighted slightly higher (25\%) to accommodate different schedules on the weekend. 
Table C-10 Hours of Operation Modeling Data by PBAPLUS

\begin{tabular}{|c|c|c|c|c|c|c|}
\hline Code & $\begin{array}{c}\text { PBAPLUS Name } \\
\text { More Specific Building } \\
\text { Activity }\end{array}$ & $\begin{array}{c}\text { Weekday } \\
\text { Center } \\
\text { Hour }\end{array}$ & $\begin{array}{l}\text { Saturday } \\
\text { Center } \\
\text { Hour }\end{array}$ & $\begin{array}{c}\text { Sunday } \\
\text { Center } \\
\text { Hour }\end{array}$ & $\begin{array}{c}\text { Small } \\
\text { Weekend } \\
\text { Center Hour }\end{array}$ & $\begin{array}{l}\text { Saturday } \\
\text { Favored }\end{array}$ \\
\hline 1 & Vacant & 13 & 13 & 13 & 10 & Yes \\
\hline 2 & $\begin{array}{l}\text { Administrative/professional } \\
\text { office }\end{array}$ & 16 & 14 & 14 & 12 & Yes \\
\hline 3 & Bank/other financial & 13 & 10 & 10 & 12 & Yes \\
\hline 4 & Government office & 13 & 13 & 15 & 12 & Yes \\
\hline 5 & $\begin{array}{l}\text { Medical office } \\
\text { (nondiagnostic) }\end{array}$ & 16 & 14 & 14 & 11 & Yes \\
\hline 6 & Mixed-use office & 16 & 14 & 14 & 11 & Yes \\
\hline 7 & Other office & 16 & 14 & 14 & 11 & Yes \\
\hline 8 & Laboratory & 13 & 11 & 13 & 11 & Yes \\
\hline 9 & Distribution/shipping center & 12 & 12 & 12 & 12 & Yes \\
\hline 10 & Nonrefrigerated warehouse & 12 & 12 & 12 & 12 & Yes \\
\hline 11 & Self-storage & 12 & 12 & 12 & 12 & Yes \\
\hline 12 & Convenience store & 13 & 14 & 12 & 12 & Yes \\
\hline 13 & $\begin{array}{l}\text { Convenience store with gas } \\
\text { station }\end{array}$ & 14 & 14 & 14 & 12 & Yes \\
\hline 14 & Grocery store/food market & 13 & 14 & 12 & 12 & Yes \\
\hline 15 & Other food sales & 13 & 14 & 12 & 12 & Yes \\
\hline 16 & Fire station/police station & 13 & 14 & 12 & 12 & Yes \\
\hline 17 & Other public order and safety & 13 & 14 & 12 & 12 & Yes \\
\hline 18 & Medical office (diagnostic) & 13 & 14 & 12 & 12 & Yes \\
\hline 19 & Clinic/other outpatient health & 13 & 14 & 12 & 12 & Yes \\
\hline 20 & Refrigerated warehouse & 13 & 14 & 12 & 12 & Yes \\
\hline 21 & Religious worship & 13 & 13 & 12 & 9 & No \\
\hline 22 & Entertainment/culture & 14 & 14 & 14 & 18 & Yes \\
\hline 23 & Library & 13 & 13 & 13 & 13 & Yes \\
\hline 24 & Recreation & 12 & 13 & 14 & 13 & Yes \\
\hline 25 & Social/meeting & 14 & 14 & 14 & 18 & Yes \\
\hline 26 & Other public assembly & 13 & 13 & 13 & 13 & Yes \\
\hline 27 & College/university & 14 & 10 & 14 & 10 & Yes \\
\hline 28 & Elementary/middle school & 14 & 14 & 14 & 10 & Yes \\
\hline 29 & High school & 14 & 14 & 14 & 10 & Yes \\
\hline 30 & Preschool/daycare & 14 & 14 & 14 & 10 & Yes \\
\hline 31 & Other classroom education & 14 & 14 & 14 & 10 & Yes \\
\hline 32 & Fast food & 18 & 18 & 18 & 16 & Yes \\
\hline 33 & Restaurant/cafeteria & 16 & 16 & 18 & 16 & Yes \\
\hline 34 & Other food service & 18 & 18 & 18 & 16 & Yes \\
\hline 35 & Hospital/inpatient health & 13 & 14 & 12 & 14 & Yes \\
\hline 36 & Nursing home/assisted living & 13 & 14 & 12 & 14 & Yes \\
\hline 37 & Dormitory/fraternity/sorority & 13 & 13 & 13 & 13 & Yes \\
\hline 38 & Hotel & 13 & 13 & 13 & 13 & Yes \\
\hline 39 & Motel or inn & 13 & 13 & 13 & 13 & Yes \\
\hline 40 & Other lodging & 13 & 13 & 13 & 13 & Yes \\
\hline
\end{tabular}




\begin{tabular}{|c|l|c|c|c|c|c|}
\hline Code & $\begin{array}{c}\text { PBAPLUS Name } \\
\text { More Specific Building } \\
\text { Activity }\end{array}$ & $\begin{array}{c}\text { Weekday } \\
\text { Center } \\
\text { Hour }\end{array}$ & $\begin{array}{c}\text { Saturday } \\
\text { Center } \\
\text { Hour }\end{array}$ & $\begin{array}{c}\text { Sunday } \\
\text { Center } \\
\text { Hour }\end{array}$ & $\begin{array}{c}\text { Small } \\
\text { Weekend } \\
\text { Center Hour }\end{array}$ & $\begin{array}{c}\text { Saturday } \\
\text { Favored }\end{array}$ \\
\hline \hline 41 & Vehicle dealership/showroom & 14 & 14 & 14 & 12 & Yes \\
\hline 42 & Retail store & 14 & 14 & 14 & 12 & Yes \\
\hline 43 & Other retail & 14 & 14 & 14 & 12 & Yes \\
\hline 44 & Post office/postal center & 14 & 14 & 14 & 12 & Yes \\
\hline 45 & Repair shop & 14 & 14 & 14 & 12 & Yes \\
\hline 46 & Vehicle service/repair shop & 14 & 14 & 14 & 12 & Yes \\
\hline 47 & Vehicle storage/maintenance & 14 & 14 & 14 & 12 & Yes \\
\hline 48 & Other service & 14 & 14 & 14 & 12 & Yes \\
\hline 49 & Other & 13 & 13 & 13 & 13 & Yes \\
\hline
\end{tabular}

Holidays also affect schedules. Holiday schedules are usually set to match the day that has the fewest open hours (typically Sunday). The holidays are listed in Table C-11. If the holiday falls on a day that the business is closed, the holiday is not moved to the following business day, but rather observed on the closed day.

Table C-11 Observed Holidays

\begin{tabular}{|l|l|}
\hline \multicolumn{1}{|c|}{ Holiday } & \multicolumn{1}{c|}{ Day of Year } \\
\hline \hline New Years Day & January 1 \\
\hline Veterans Day & November 11 \\
\hline Christmas & December 25 \\
\hline Independence Day & July 4 \\
\hline Martin Luther King Day & Third Monday in January \\
\hline Presidents Day & Third Monday in February \\
\hline Memorial Day & Last Monday in May \\
\hline Labor Day & First Monday in September \\
\hline Columbus Day & Second Monday in October \\
\hline Thanksgiving & Fourth Thursday in November \\
\hline
\end{tabular}

Each building's lighting schedule uses the hours of operation schedule to determine the timing of lights. The lighting schedule was modeled from the hours of operation schedule with maximum and minimum fractions determined from CBECS variables (see Table C-12). The schedule values area also scaled by an additional factor for diversity (see Table C-27).

Table C-12 Lighting Schedule Modeling

\begin{tabular}{|c|l|l|}
\hline CBECS Variable & \multicolumn{1}{|c|}{ Description } & \multicolumn{1}{c|}{ Notes } \\
\hline \hline RDLTNF & $\begin{array}{l}\text { Lighting reduced during off hours } \\
1-\text { Yes } \\
2-\text { No }\end{array}$ & If 0 then defaults to other schedule \\
\hline LTNHRP & Percent lit when closed & Used for minimum fraction for schedule \\
\hline LTOHRP & Percent lit when open & Used for maximum fraction for schedule \\
\hline
\end{tabular}

The building equipment schedule used to model plug and process electricity use is based on the operation schedule created first. When occupied, the equipment fraction is assumed to be at a maximum of 0.95 . 
When unoccupied, the equipment fraction is assumed to be at one of the minimum fractions (see Table C14). Using the variable RDPFEQ in Table C-13, the fractions were applied (see Table C-14), by assumption.

Table C-13 Plug and Process Load Schedule Modeling

\begin{tabular}{|c|c|c|}
\hline CBECS Variable & Description (Weight of Occurrence) & Notes \\
\hline RDOFEQ & $\begin{array}{l}\text { Equipment turned off during off hours } \\
0 \text { - Not reported }(40 \%) \\
1 \text { - Always }(32 \%) \\
2 \text { - Sometimes }(16 \%) \\
3 \text { - Never }(10 \%) \\
4 \text { - Computers are powered down }(2 \%)\end{array}$ & $\begin{array}{l}\text { If } 0 \text { then assumed to be } 1 \\
- \text { always turned off } \\
\text { (because } 1 \text { is the most } \\
\text { common response) }\end{array}$ \\
\hline
\end{tabular}

Table C-14 Plug and Process Load Fractions

\begin{tabular}{|l|c|}
\hline \multicolumn{1}{|c|}{ RDOFEQ Variable } & Plug and Process Load Minimum Fraction \\
\hline Not reported & 0.10 \\
\hline Always & 0.10 \\
\hline Sometimes & 0.40 \\
\hline Never & 0.95 \\
\hline Computers are powered down & 0.80 \\
\hline
\end{tabular}

Heating and cooling thermostatic set points were set based on the CBECS variables listed in Table C-15.

Table C-15 Variables for Heating and Cooling Set Points

\begin{tabular}{|c|c|c|}
\hline CBECS Variable & Description & Notes \\
\hline RDCLNF & $\begin{array}{l}\text { Cooling reduced in } 24 \text { hours } \\
1-\text { Yes } \\
2-\text { No }\end{array}$ & $\begin{array}{l}\text { If } 0 \text { then assumed to be no } \\
\text { (because } 2 \text { is the most common } \\
\text { response) }\end{array}$ \\
\hline RDHTNF & $\begin{array}{l}\text { Heating reduced in } 24 \text { hours } \\
1-\text { Yes } \\
2-\text { No }\end{array}$ & $\begin{array}{l}\text { If } 0 \text { then assumed to be no } \\
\text { (because } 2 \text { is the most common } \\
\text { response) }\end{array}$ \\
\hline HWRDCL & $\begin{array}{l}\text { How reduce cooling } \\
1-\text { Time-clock thermostat } \\
2-\text { Manually reset } \\
3-\text { Part of EMCS }\end{array}$ & If 0 then not reduced (not used) \\
\hline HWRDHT & $\begin{array}{l}\text { How reduce heating } \\
1 \text { - Time-clock thermostat } \\
2-\text { Manually reset } \\
3 \text { - Part of EMCS }\end{array}$ & If 0 then not reduced \\
\hline HTLS50 & $\begin{array}{l}\text { Heated to lower than } 50^{\circ} \mathrm{F} \\
1-\text { Yes } \\
2-\text { No }\end{array}$ & $\begin{array}{l}\text { If yes then set to } 50^{\circ} \mathrm{F} \text { and } \\
\text { nonrefrigerated warehouse }\end{array}$ \\
\hline
\end{tabular}

The maximum heating and cooling set points were assigned based on the PBA during operating hours. Setback is used only if stated in CBECS. The heating and cooling set points were always assumed to be the same, except in vacant buildings. They were defined as in Table C-16. However, if the building was heated to lower than $50^{\circ} \mathrm{F}\left(10^{\circ} \mathrm{C}\right)$, the maximum heating set point and setback were set to $50^{\circ} \mathrm{F}\left(10^{\circ} \mathrm{C}\right)$. 
Table C-16 Thermostat Set Point Schedule Modeling

\begin{tabular}{|l|c|c|c|c|}
\hline $\begin{array}{c}\text { PBAPLUS } \\
\text { Name }\end{array}$ & $\begin{array}{c}\text { Heating Set Point } \\
\left({ }^{\circ} \mathbf{C}\right)\end{array}$ & $\begin{array}{c}\text { Heating Setback } \\
\left({ }^{\circ} \mathbf{C}\right)\end{array}$ & $\begin{array}{c}\text { Cooling Set Point } \\
\left({ }^{\circ} \mathbf{C}\right)\end{array}$ & $\begin{array}{c}\text { Cooling Setback } \\
\left({ }^{\circ} \mathbf{C}\right)\end{array}$ \\
\hline \hline Vacant & 19 & 13 & 24 & 33 \\
\hline All others & 21 & 13 & 24 & 33 \\
\hline
\end{tabular}

The HVAC operation schedule was the same as the hours of operation schedule unless the building's heating or cooling was reduced during the night.

Metabolic activity level is used to model thermal comfort and requires scheduled values (Table C-17). 
Table C-17 Activity Level Schedule Modeling

\begin{tabular}{|c|c|c|}
\hline PBA Code & PBAPLUS Name & Activity Level (W) \\
\hline 1 & Vacant & 120 \\
\hline 2 & Administrative/professional office & 120 \\
\hline 3 & Bank/other financial & 120 \\
\hline 4 & Government office & 120 \\
\hline 5 & Medical office (nondiagnostic) & 120 \\
\hline 6 & Mixed-use office & 120 \\
\hline 7 & Other office & 120 \\
\hline 8 & Laboratory & 140 \\
\hline 9 & Distribution/shipping center & 140 \\
\hline 10 & Nonrefrigerated warehouse & 140 \\
\hline 11 & Self-storage & 140 \\
\hline 12 & Convenience store & 120 \\
\hline 13 & Convenience store with gas station & 120 \\
\hline 14 & Grocery store/food market & 120 \\
\hline 15 & Other food sales & 120 \\
\hline 16 & Fire station/police station & 120 \\
\hline 17 & Other public order and safety & 120 \\
\hline 18 & Medical office (diagnostic) & 120 \\
\hline 19 & Clinic/other outpatient health & 120 \\
\hline 20 & Refrigerated warehouse & 120 \\
\hline 21 & Religious worship & 120 \\
\hline 22 & Entertainment/culture & 120 \\
\hline 23 & Library & 80 \\
\hline 24 & Recreation & 250 \\
\hline 25 & Social/meeting & 100 \\
\hline 26 & Other public assembly & 120 \\
\hline 27 & College/university & 120 \\
\hline 28 & Elementary/middle school & 120 \\
\hline 29 & High school & 120 \\
\hline 30 & Preschool/daycare & 120 \\
\hline 31 & Other classroom education & 120 \\
\hline 32 & Fast food & 120 \\
\hline 33 & Restaurant/cafeteria & 120 \\
\hline 34 & Other food service & 120 \\
\hline 35 & Hospital/inpatient health & 100 \\
\hline 36 & Nursing home/assisted living & 100 \\
\hline 37 & Dormitory/fraternity/sorority & 120 \\
\hline 38 & Hotel & 120 \\
\hline 39 & Motel or inn & 120 \\
\hline 40 & Other lodging & 120 \\
\hline 41 & Vehicle dealership/showroom & 120 \\
\hline 42 & Retail store & 120 \\
\hline 43 & Other retail & 120 \\
\hline
\end{tabular}




\begin{tabular}{|c|l|c|}
\hline PBA Code & \multicolumn{1}{|c|}{ PBAPLUS Name } & Activity Level (W) \\
\hline 44 & Post office/postal center & 120 \\
\hline 45 & Repair shop & 140 \\
\hline 46 & Vehicle service/repair shop & 140 \\
\hline 47 & Vehicle storage/maintenance & 140 \\
\hline 48 & Other service & 120 \\
\hline 49 & Other & 120 \\
\hline
\end{tabular}

\section{C.6 Floor Area and Number of Floors}

CBECS provides values for total floor area (SQFT8) and number of floors (NFLOOR8). Therefore, the mapping is often trivial and these are directly translated to FloorArea and NumFloors in the data model.

CBECS uses special values for NFLOOR8 to mask the identity of actual buildings with large numbers of floors. If NFLOOR8 has the value 991, we used a uniform probability distribution to select the number of floors at random from 15 to 25, inclusive. If NFLOOR8 has the value 992, we used a uniform probability distribution to select the number of floors at random from 26 to 50 , inclusive.

CBECS value for number of floors includes basements, parking levels, and any other floors below grade level. However, in the EnergyPlus modeling, we do not model below grade levels and assume that all the floors are aboveground.

\section{C.7 Aspect Ratio and Rotation}

The aspect ratio is defined as the east-west length of the building divided by the north-south width of the building. Obviously, this definition is unique only before the building is rotated; that is, when Rotation = 0 . The aspect ratio for each building was modeled from the 2003 CBECS variables:

- Building shape (BLDSHP8), which can take the following values (\% sector floor area):

○ 00 not ascertained in survey $(10.1 \%)$

$\circ \quad 01$ square $(8.5 \%)$

○ 02 wide rectangle $(48.8 \%)$

○ 03 narrow rectangle $(7.0 \%)$

○ 04 rectangle square with courtyard (3.7\%)

○ 05 "H" shaped (3.0\%)

○ 06 "U” shaped (2.5\%)

○ 07 "E" shaped (1.3\%)

○ 08 "T" shaped (2.1\%)

○ 09 "L" shaped (6.2\%)

○ 10 "+" or cross shaped (1.8\%)

○ 11 other shape $(4.9 \%)$

- Glass sides receive most sunlight (SUNGLS8).

To simplify the generation of full energy models, we use only rectangular forms and neglect the other general shapes of courtyards, "H", "U", "E", "T", "L", or "+". These forms are used in $20 \%$ of the buildings. Such forms are accounted for by shifting to higher aspect ratios to model increased perimeter area for a given floor area as listed in Table C-18. 
Table C-18 Aspect Ratio Assignments by Building Shape

\begin{tabular}{|c|l|l|l|}
\hline $\begin{array}{c}\text { BLDSHP8 } \\
\text { Code }\end{array}$ & \multicolumn{1}{|c|}{ Shape } & $\begin{array}{c}\text { Probability Distribution } \\
\text { for Aspect Ratio }\end{array}$ & Source \\
\hline 00 & Not ascertained & Uniform on [1..4] & Assumption \\
\hline 01 & Square & Uniform on [1..1.2] & Assumption \\
\hline 02 & Wide rectangle & Uniform on [1.2..2] & Assumption \\
\hline 03 & Narrow rectangle & Uniform on [2..6] & Assumption \\
\hline 04 & Rectangle/square with courtyard & Uniform on [2.0.5.0] & Assumption \\
\hline 05 & "H" shaped & Uniform on [3..6.0] & Assumption \\
\hline 06 & "U" shaped & Uniform on [2..5] & Assumption \\
\hline 07 & "E" shaped & Uniform on [2..5] & Assumption \\
\hline 08 & "T" shaped & Uniform on [1.5..5] & Assumption \\
\hline 09 & "L" shaped & Uniform on [1.5..4] & Assumption \\
\hline 10 & "+" or cross shaped & Uniform on [2.0..6] & Assumption \\
\hline 11 & other & Uniform on [1..4] & Assumption \\
\hline
\end{tabular}

Rotation is assigned on [0..360) randomly by using uniform probability distribution.

\section{C.8 Perimeter Depth}

The perimeter depth is defined as the distance from the outside wall to the interior core zone. This value was fixed at $15 \mathrm{ft}(4.57 \mathrm{~m})$ following customary energy modeling practice. This value is also selected, because with this we have more faith in the capability of the split-flux models for daylighting.

\section{C.9 Floor-to-Floor Height}

CBECS does not provide data about the floor-to-floor height, which is defined as the distance from the top of one floor to the top of the next higher floor (includes any plenum spaces). In our modeling, the building is assumed to have equal floor-to-floor heights on all levels. This value is therefore generated at random from assumed ranges. Table C-19 documents how these rules were organized by PBA. 
Table C-19 Floor-to-Floor Height Assignments by Subsector

\begin{tabular}{|l|l|l|l|}
\hline PBA Code & \multicolumn{1}{|c|}{ Subsector (PBA) } & Probability Distribution & \multicolumn{1}{c|}{ Source } \\
\hline \hline 1 & Vacant & Uniform on [10..20] ft & Assumption \\
\hline 2 & Office/professional & Uniform on [11..15] ft & Assumption \\
\hline 4 & Laboratory & Uniform on [13..17] ft & Assumption \\
\hline 5 & Nonrefrigerated warehouse & Uniform on [15..20] ft & Assumption \\
\hline 6 & Food sales & Uniform on [15..20] ft & Assumption \\
\hline 7 & Public order and safety & Uniform on [13..17] ft & Assumption \\
\hline 8 & Outpatient health care & Uniform on [11..15] ft & Assumption \\
\hline 11 & Refrigerated warehouse & Uniform on [15..20] ft & Assumption \\
\hline 12 & Religious worship & Uniform on [15..25] ft & Assumption \\
\hline 13 & Public assembly & Uniform on [15..25] ft & Assumption \\
\hline 14 & Education & Uniform on [11..15] ft & Assumption \\
\hline 15 & Food service & Uniform on [11..15] ft & Assumption \\
\hline 16 & Inpatient health care & Uniform on [11..15] ft & Assumption \\
\hline 17 & Skilled nursing & Uniform on [10..13] ft & Assumption \\
\hline 18 & Lodging & Uniform on [10..13] ft & Assumption \\
\hline 23 & Strip shopping mall & Uniform on [15..20] ft & Assumption \\
\hline 24 & Enclosed mall & Uniform on [15..25] ft & Assumption \\
\hline 25 & Retail (except malls) & Uniform on [15..20] ft & Assumption \\
\hline 26 & Service & Uniform on [15..20] ft & Assumption \\
\hline 91 & Other & Uniform on [15..20] ft & Assumption \\
\hline
\end{tabular}

\section{C.10 Plenum Height}

CBECS provides no information on the height of plenum spaces. This value is zero in building types that do not include suspended ceilings. The floor-to-floor height minus the plenum height results in the zone ceiling height for the occupied space. Earlier efforts to develop the current methodology included plenums in some of the model geometries. However, the final set of models had no plenums.

\section{C.11 Glazing Geometry}

The locations and sizes of windows in the building need to be known and are referred to here as glazing geometry. The most important glazing geometry parameter is the glazing fraction, which is defined as the total (above-grade) wall area divided by the total visible window area (for each orientation). The schema for window form used in this study is continuous bands of windows for each floor and each orientation. Although real buildings will often have individual windows, continuous horizontal bands are used to simplify the EnergyPlus models and avoid excessive simulation run times.

2003 CBECS provides the following data related to glazing geometry:

- Percent exterior glass (GLSSPC8)

$$
\begin{array}{lll}
\circ & 0 & \text { no data } \\
\circ & 1 & 10 \% \text { or less } \\
\circ & 2 & 11 \% \text { to } 25 \% \\
\circ & 3 & 16 \% \text { to } 50 \% \\
\circ & 4 & 51 \% \text { to } 75 \% \\
\circ & 5 & 76 \% \text { to } 100 \%
\end{array}
$$

- $\quad$ Equal glass on all sides (EQGLSS8) 
$\begin{array}{ll}\circ & 0 \text { no data } \\ \circ & 1 \text { yes } \\ \circ & 2 \text { no }\end{array}$

- Glass sides receive most sunlight (SUNGLS8)
○ 0 no data
○ 1 "more glass area"
○ 2 "less glass area"
○ 3 "about the same"

- Skylights and atriums designed for lighting (SKYLT8).

If data are reported for percent exterior glass, glazing fractions are assigned to be within the reported category. A uniform probability distribution is used to select a specific glazing fraction within the range specified by each category according to Table C-20.

Table C-20 Glazing Fraction Assignments

\begin{tabular}{|c|l|l|}
\hline GLSSPC8 Code & \multicolumn{1}{|c|}{ Category } & Probability Distribution \\
\hline 00 & Not ascertained & See Table C-21 \\
\hline 01 & $10 \%$ or less & Uniform on [0.0..0.1] \\
\hline 02 & $11 \%$ to $25 \%$ & Uniform on [0.11..0.25] \\
\hline 03 & $16 \%$ to $50 \%$ & Uniform on [0.26..0.50] \\
\hline 04 & $51 \%$ to $75 \%$ & Uniform on $[0.51 . .0 .75]$ \\
\hline 05 & $76 \%$ to $100 \%$ & Uniform on $[0.76 . .0 .98]$ \\
\hline
\end{tabular}

For cases where the CBECS data about percent exterior glass are missing (GLSSPC $8=0$ ), we have no data to determine the glazing fraction. For these cases we used assignments developed from Huang and Franconi (1999) and assumptions as shown in Table C-21.

Table C-21 Glazing Fraction Assignments by PBA (Used When No Data Are Available for Percent Exterior Glass)

\begin{tabular}{|c|c|c|c|}
\hline $\begin{array}{l}\text { PBA } \\
\text { Code }\end{array}$ & PBA Name & Rule & Source \\
\hline 1 & Vacant & $\begin{array}{l}\text { Post-1980: } \\
\text { If floor area } \geq 25,000 \mathrm{ft}^{2} \text { then } 0.5 \\
\text { If floor area }<25,000 \mathrm{ft}^{2} \text { then } 0.15 \\
\text { Pre-1980: } \\
\text { If floor area } \geq 25,000 \mathrm{ft}^{2} \text { then } 0.4 \\
\text { If floor area }<25,000 \mathrm{ft}^{2} \text { then } 0.2\end{array}$ & $\begin{array}{l}\text { Assumed to } \\
\text { match office } \\
\text { from Huang } \\
\text { and Franconi } \\
1999\end{array}$ \\
\hline 2 & Office/professional & $\begin{array}{l}\text { Post-1980: } \\
\text { If floor area } \geq 25,000 \mathrm{ft}^{2} \text { then } 0.5 \\
\text { If floor area }<25,000 \mathrm{ft}^{2} \text { then } 0.15 \\
\text { Pre-1980: } \\
\text { If floor area } \geq 25,000 \mathrm{ft}^{2} \text { then } 0.4 \\
\text { If floor area }<25,000 \mathrm{ft}^{2} \text { then } 0.2\end{array}$ & $\begin{array}{l}\text { Huang and } \\
\text { Franconi } 1999\end{array}$ \\
\hline 4 & Laboratory & 0.10 & Assumption \\
\hline 5 & $\begin{array}{l}\text { Nonrefrigerated } \\
\text { warehouse }\end{array}$ & $\begin{array}{l}\text { If post- } 1980 \text { then } 0.03 \\
\text { If pre-1980 then } 0.06\end{array}$ & $\begin{array}{l}\text { Huang and } \\
\text { Franconi } 1999\end{array}$ \\
\hline 6 & Food sales & 0.15 & $\begin{array}{l}\text { Huang and } \\
\text { Franconi } 1999\end{array}$ \\
\hline 7 & Public order and safety & $\begin{array}{l}\text { Post-1980: } \\
\quad \text { If floor area } \geq 25,000 \mathrm{ft}^{2} \text { then } 0.5\end{array}$ & $\begin{array}{l}\text { Assumed to } \\
\text { match office }\end{array}$ \\
\hline
\end{tabular}




\begin{tabular}{|c|c|c|c|}
\hline $\begin{array}{l}\text { PBA } \\
\text { Code }\end{array}$ & PBA Name & Rule & Source \\
\hline & & $\begin{array}{l}\text { If floor area }<25,000 \mathrm{ft}^{2} \text { then } 0.15 \\
\text { Pre-1980: } \\
\text { If floor area } \geq 25,000 \mathrm{ft}^{2} \text { then } 0.4 \\
\text { If floor area }<25,000 \mathrm{ft}^{2} \text { then } 0.2\end{array}$ & $\begin{array}{l}\text { from Huang } \\
\text { and Franconi } \\
1999\end{array}$ \\
\hline 8 & Outpatient health care & 0.25 & $\begin{array}{l}\text { Huang and } \\
\text { Franconi } 1999 \\
\text { hospital }\end{array}$ \\
\hline 11 & Refrigerated warehouse & 0.0 & Assumption \\
\hline 12 & Religious worship & 0.20 & Assumption \\
\hline 13 & Public assembly & $\begin{array}{l}\text { Post-1980: } \\
\text { If floor area } \geq 25,000 \mathrm{ft}^{2} \text { then } 0.5 \\
\text { If floor area }<25,000 \mathrm{ft}^{2} \text { then } 0.15 \\
\text { Pre-1980: } \\
\text { If floor area } \geq 25,000 \mathrm{ft}^{2} \text { then } 0.4 \\
\text { If floor area }<25,000 \mathrm{ft}^{2} \text { then } 0.2\end{array}$ & $\begin{array}{l}\text { Assumed to } \\
\text { match office } \\
\text { from Huang } \\
\text { and Franconi } \\
1999\end{array}$ \\
\hline 14 & Education & $\begin{array}{l}\text { If post- } 1980 \text { then } 0.18 \\
\text { If pre-1980 then } 0.27\end{array}$ & $\begin{array}{l}\text { Huang and } \\
\text { Franconi } 1999\end{array}$ \\
\hline 15 & Food service & $\begin{array}{l}\text { If post- } 1980 \text { then } 0.175 \\
\text { If pre- } 1980 \text { then } 0.25\end{array}$ & $\begin{array}{l}\text { Huang and } \\
\text { Franconi } 1999 \\
\text { (average of } \\
\text { restaurants) }\end{array}$ \\
\hline 16 & Inpatient health care & 0.25 & $\begin{array}{l}\text { Huang and } \\
\text { Franconi } 1999 \\
\text { hospital }\end{array}$ \\
\hline 17 & Skilled nursing & 0.25 & $\begin{array}{l}\text { Huang and } \\
\text { Franconi } 1999 \\
\text { hospital }\end{array}$ \\
\hline 18 & Lodging & $\begin{array}{l}\text { Post-1980: } \\
\text { If floor area } \geq 25,000 \mathrm{ft}^{2} \text { then } 0.35 \\
\text { If floor area }<25,000 \mathrm{ft}^{2} \text { then } 0.21 \\
\text { Pre-1980: } \\
\text { If floor area } \geq 25,000 \mathrm{ft}^{2} \text { then } 0.29 \\
\text { If floor area }<25,000 \mathrm{ft}^{2} \text { then } 0.24\end{array}$ & $\begin{array}{l}\text { Huang and } \\
\text { Franconi } 1999\end{array}$ \\
\hline 25 & Retail (except malls) & 0.15 & $\begin{array}{l}\text { Huang and } \\
\text { Franconi } 1999\end{array}$ \\
\hline 26 & Service & 0.15 & Assumption \\
\hline 91 & Other & 0.20 & Assumption \\
\hline
\end{tabular}

Once the overall glazing fraction has been determined, the CBECS variable for "equal glass on all sides" (EQGLSS8) is used, if possible, to further refine the distribution of glazing on the cardinal directions. If the value of EQGLSS8 is " 0 " (not available) or " 1 " (yes), we used the same glazing fraction on all four cardinal directions. If the value of EQGLSS8 is "2" (no), we distributed the glazing fraction to be different in the different cardinal directions in the following manner. The CBECS variable "glass sides receive most sunlight" (SUNGLS8) was assumed to indicate a tendency for the relative glazing fractions along the south-facing facade. If the value of SUNGLS8 was "1" (more glass area), we increased the glazing fraction on the south facade using,

$$
f_{g l s, \text { south }}=\operatorname{MIN}\left(f_{g l s, \text { south }}+f_{g l s, \text { south }} / 2, \quad f_{g l s, \text { south }}+\left(1-f_{g l s, \text { south }}\right) / 2\right) \text {. }
$$

If the value of SUNGLS8 was " 2 " (less glass area), we decreased the glazing fraction on the south facade using,

$$
f_{g l s, \text { south }}=f_{\text {gls,south }}-f_{g l s, \text { south }} / 2 \text {. }
$$


If the value of SUNGLS8 was " 0 " or " 3 ", the SUNGLS8 variable does not affect the glazing fraction allocations, and unequal glazing was modeled by picking a facade orientation at random and then increasing the glazing on that facade using,

$$
f_{g l s, \text { south }}=\operatorname{MIN}\left(f_{g l s, \text { south }}+f_{g l s, \text { south }} / 4, \quad f_{g l s, \text { south }}+\left(1-f_{g l s, \text { south }}\right) / 4\right)
$$

At this point in the assignments and geometry generation, we have not yet calculated the overall areas of the facade in the cardinal directions. This makes it difficult to use a scheme that would preserve glazed areas. The areas could be computed at this point, but these calculations are being done much later in the process. Given the vagaries of the glazing fraction value, it does not seem necessary to ensure that the overall value is totally preserved when redistributing.

The data model requires two additional parameters for glazing geometry: GlazingSillHeight and GlazingEdgeOffset. The sill height is the distance from each floor to where the window starts. This value is fixed by assumption at $3.6 \mathrm{ft}(1.1 \mathrm{~m})$, except for high glazing fractions where lower sills are required to provide enough area for higher glazing fractions. The next value is the edge offset, which is the length of clear wall along the window jambs (also required to ensure that windows are smaller than the walls in which they are located). This value is fixed by assumption at $0.16 \mathrm{ft}(0.05 \mathrm{~m})$.

\section{C.12 Construction}

This mapping selects sets of constructions in the data model. CBECS provides PBA and year of construction along with a number of variables that provide some information about the envelope, including:

- Insulation upgrade (RENINS8)

○ 00 not known

○ 01 yes

○ 02 no

- Wall construction material (WLCNS8)

○ 01 brick, stone or stucco $(51 \%)$

○ 02 precast concrete panels (10\%)

○ 03 concrete block or poured concrete $(17 \%)$

○ 04 siding, shingles, tiles, or shakes (6\%)

○ 05 sheet metal panels (12\%)

○ 06 window or vision glass $(1.6 \%)$

○ 07 decorative or construction glass $(0.8 \%)$

○ 08 no one major type $(0.6 \%)$

○ 09 other $(0.8 \%)$

- Roof construction material (RFCNS8)

○ 01 built up (33\%)

○ 02 slate or tile shingles (4\%)

○ 03 wood shingles, shakes, or other wood (1.4\%)

○ 04 asphalt, fiberglass, or other shingles (16\%) 
○ 05 metal surfacing (18\%)

○ 06 plastic, rubber, or synthetic sheeting (23\%)

○ 07 concrete $(3.4 \%)$

○ 08 no one major type $(0.9 \%)$

○ 09 other $(0.9 \%)$

- $\quad$ Tinted window glass (TINT8)

- Window glass type (WINTYP8)

- Window replacement (RENWIN8)

- Reflective window glass (REFL8)

With these data, and the activity and location (determined earlier), the construction types for the thermal envelope are assigned in the following manner.

The year of construction variable (YRCON8) is examined and sorted into vintages - pre-1980 and post1980. This categorization was selected to align with Huang and Franconi (1999) to use their data in assignments. If the insulation was renovated (RENINS8 = yes), the vintage was set to post-1980 regardless of the year of construction.

The vintage is used with the CBECS variables for census division (CENDIV8) and PBA8 to assign construction R-values according to data from Huang and Franconi (1999). These data were presented in Table 2-6 and Table 2-7.

Locations were mapped based on CDDs and HDDs. In Huang and Franconi's document, they split Climate Zone 3 into two parts (north and south). This climate zone had 4,000 to 5,499 HDDs and fewer than 2,000 CDDs. To disaggregate this climate zone, the north was assumed to have more than 4,750 HDDs, and the south was assumed to have 4,750 or fewer HDDs.

The type of windows installed was based on the four CBECS variables listed in Table C-22.

Table C-22 Variables Used To Determine Window Constructions

\begin{tabular}{|c|l|}
\hline Variable ID & Description \\
\hline \hline \multirow{3}{*}{ TINT8 } & Tinted window glass \\
& $1-$ Yes \\
& $2-$ No \\
\hline & Window glass type \\
& $1-$ Single layer glass \\
WINTYP8 & $2-$ Multi-layer glass \\
& $3-$ Combination of both \\
& $4-$ No windows \\
\hline \multirow{3}{*}{ RENWIN8 } & Window replacement \\
& $1-$ Yes \\
& $2-$ No \\
\hline \multirow{3}{*}{ REFL8 } & Reflective window glass \\
& $1-$ Yes \\
& $2-$ No \\
\hline
\end{tabular}

The values for RENWIN8 were not used because the variable WINTYP8 included the renovated window constructions. Based on the three variables, a $3 \times 2$ matrix was created to assign window constructions. 
The six window types are either single or double pane and have clear, tinted, or reflective solar optical properties.

\section{C.13 People Density}

CBECS provides data about the number of workers (NWKER8), the total square feet (SQFT8), and various numbers for capacities (RWSEAT8, PBSEAT8, EDSEAT8, FDSEAT8, and HCBED8). The data model uses the PeopleDensity element, which is defined as the number of people in the space per every $100 \mathrm{~m}^{2}\left(1076 \mathrm{ft}^{2}\right)$. ASHRAE 90.1-1989 Section 13 provides recommendations for occupancy density. Table C-23 shows how the occupancy assignments are made for each subsector. We generally use the number of workers specified in CBECS, unless nonemployees as well as employees are expected to be in the buildings. The people density assignment for vacant buildings is used to size the HVAC systems, and schedules are used to zero out the number of occupants.

Table C-23 People Density Assignments

\begin{tabular}{|c|c|c|c|}
\hline $\begin{array}{l}\text { PBA } \\
\text { Code }\end{array}$ & Subsector & Assignment Rules & Source \\
\hline$\overline{1}$ & Vacant & $200 \mathrm{ft}^{2} /$ person & Assumption \\
\hline 2 & Office/professional & NWKER8/SQFT8 * Conversion & 2003 CBECS \\
\hline 4 & Laboratory & NWKER8/SQFT8 * Conversion & 2003 CBECS \\
\hline 5 & Nonrefrigerated warehouse & NWKER8/SQFT8 * Conversion & 2003 CBECS \\
\hline 6 & Food sales & $300 \mathrm{ft}^{2} /$ person & $\begin{array}{l}\text { ASHRAE 90.1-1989 } \\
\text { (retail) }\end{array}$ \\
\hline 7 & Public order and safety & NWKER8/SQFT8 * Conversion & 2003 CBECS \\
\hline 8 & Outpatient health care & $200 \mathrm{ft}^{2} /$ person & $\begin{array}{l}\text { ASHRAE 90.1-1989 } \\
\text { w/addendum } \\
\text { (Health/Institutional) }\end{array}$ \\
\hline 11 & Refrigerated warehouse & (NWKER8/SQFT8) * Conversion & 2003 CBECS \\
\hline 12 & Religious worship & $\begin{array}{l}\text { (NWKER8 + 0.5*RWSEAT8)/SQFT8 * } \\
\text { Conversion }\end{array}$ & Assumption \\
\hline 13 & Public assembly & $\begin{array}{l}\text { (NWKER8 +0.5*PBSEAT8)/SQFT8 * } \\
\text { Conversion }\end{array}$ & $\begin{array}{l}2003 \text { CBECS; } \\
\text { Assumption }\end{array}$ \\
\hline 14 & Education & $\begin{array}{l}\text { (NWKER8 + 0.9*EDSEAT8)/SQFT8 * } \\
\text { Conversion }\end{array}$ & 2003 CBECS \\
\hline 15 & Food service & $\begin{array}{l}\text { (NWKER8 + 0.8*FDSEAT8)/SQFT8 * } \\
\text { Conversion }\end{array}$ & $\begin{array}{l}1999 \text { CBECS; } \\
\text { Assumption }\end{array}$ \\
\hline 16 & Inpatient health care & $\begin{array}{l}\text { (NWKER8 + 0.8*HCBED8)/SQFT8 * } \\
\text { Conversion }\end{array}$ & $\begin{array}{l}2003 \text { CBECS; } \\
\text { Assumption }\end{array}$ \\
\hline 17 & Skilled nursing & $200 \mathrm{ft}^{2} /$ person & $\begin{array}{l}\text { ASHRAE } 90.1-1989 \\
\text { w/addendum } \\
\text { (Health/Institutional) }\end{array}$ \\
\hline 18 & Lodging & $200 \mathrm{ft}^{2} /$ person & $\begin{array}{l}\text { ASHRAE } 90.1-1989 \\
\text { w/addendum } \\
\text { (Health/Institutional) }\end{array}$ \\
\hline 25 & Retail (except malls) & $300 \mathrm{ft}^{2} /$ person & $\begin{array}{l}\text { ASHRAE 90.1-1989 } \\
\text { (Retail) }\end{array}$ \\
\hline 26 & Service & NWKER8/SQFT8 * Conversion & 2003 CBECS \\
\hline 91 & Other & NWKER8/SQFT8 * Conversion & 2003 CBECS \\
\hline
\end{tabular}

\section{C.14 Plug and Process Electricity Intensity}

Establishing levels for plug and process loads for whole building energy modeling is very difficult. These energy uses are not regulated as part of building design, and little information is available to help model these loads. The plug and process intensity is used in conjunction with schedules to determine energy use in the EnergyPlus models. For this study, we used modeling based on CBECS variables to determine plug and process where possible, and iterated assumed power densities and probabilistic assignments. 
CBECS variables include the number of some types of plug and process electrical equipment.

- Number of computers (PCNUM8)

- Flat screen monitors (FLAT8)

○ Computer area percent (PCRMP8)

- Computers used (PCTERM8)

- $\quad$ Number of servers (SRVNUM8)

○ Dedicated servers used (SERVER8)

- Number of cash registers (RGSTRN8)

- Number of photocopiers (COPRN8)

- Number of printers (PRNTRN8)

○ Types of printers (PRNTYP8)

- Number of residential refrigerators (RFGRSN8)

- Number of vending machines (RFGVNN8)

- Number of elevators (NELVTR8)

- Number of escalators (NESLTR8)

The CBECS variables for number of devices can be combined with the electrical power draws of such devices when active to develop estimates for the intensity of plug and process loads. Roth et al. (2002) and others have developed estimates of the energy consumed by office and telecommunications equipment. The Roth study and others like it contain a bottom-up assessment of office equipment that uses data about the power draws and usage patterns. We selected data from Roth et al. (2002) for the nominal power of various pieces of office equipment, as listed in Table C-24.

Table C-24 Mean Nominal Peak Power Levels of Surveyed Devices

\begin{tabular}{|l|c|l|c|}
\hline \multicolumn{1}{|c|}{ Listed Item } & CBECS Variable & \multicolumn{1}{|c|}{ Data Source } & $\begin{array}{c}\text { Nominal Mean Power } \\
\text { (W) }\end{array}$ \\
\hline Personal computers & PCNUM8 & Roth et al. 2002 & 55 \\
\hline $\begin{array}{l}\text { Personal computer monitors (CRTs } \\
\text { when FLAT8 = no or missing) }\end{array}$ & PCNUM8 & Roth et al. 2002 & 90 \\
\hline $\begin{array}{l}\text { Personal computer monitors } \\
\text { (flat screens when FLAT8 = yes) }\end{array}$ & PCNUM8 & Roth et al. 2002 & 25 \\
\hline Servers & SRVNUM8 & Roth et al. 2002 & 650 \\
\hline Point of sale (cash registers) & RGSTRN8 & Roth et al. 2002 & 50 \\
\hline Printers (laser) & PRNTRN8 & Roth et al. 2002 & 263 \\
\hline Printers (inkjet) & PRNTRN8 & Roth et al. 2002 & 42.5 \\
\hline Copy machines & COPRN8 & Roth et al. 2002 & 660 \\
\hline Residential refrigerators & RFGRSN8 & Assumption & 350 \\
\hline Vending machines & RFGVNN8 & $\begin{array}{l}\text { Assumption; } \\
\text { see ADL (1993) }\end{array}$ & 450 \\
\hline Escalators & NESLTR8 & TIAX (2006) & 4,671 \\
\hline Elevators & NELVTR8 & TIAX (2006) & 10,000 \\
\hline
\end{tabular}


The nominal mean power levels listed in Table C-24 are used in the first step of the method for assigning plug and process power densities. The power density of surveyed devices, $P D_{s d}$, is calculated using,

$$
P D_{s d}=\frac{\left(\begin{array}{c}
P_{P C} R_{P C} P C N U M 8+P_{\text {mon }} R_{\text {mon }} P C N U M 8+P_{\text {server }} R_{\text {server }} S R V N U M 8+P_{P O S} R_{P O S} R G S T R N 8 \\
+P_{P R N} R_{P R N} P R N T R N 8+P_{\text {copy }} R_{\text {copy }} C O P R N 8+P_{r f} R_{r f} R F G R S N 8+P_{\text {vend }} R_{\text {vend }} R F G V N N 8 \\
+P_{E s c l} R_{E s c l} N E S L T R 8+P_{E l e v} R_{\text {Elev }} N E L V T R 8
\end{array}\right)}{S Q F T 8}
$$

where,

$\boldsymbol{P}$ is the nominal peak power for each of the surveyed devices listed in Table C-24,

$\boldsymbol{R}$ is a Gaussian random number about 1.0 with a standard deviation of 0.05 that is introduced to model variation in such power levels.

The modeled plug and process peak power density, $P$, is then modeled from the power density of surveyed devices using the linear formulation,

$$
P=\left(c_{s d} P D_{s d}+P D_{m i s c}\right) * d
$$

where,

$\boldsymbol{c}_{s d}$ is a coefficient used to scale power density from $P D_{s d}$

$P D_{\text {misc }}$ is the power density of devices that are independent of the devices included in the survey. Values for $P D_{m i s c}$ are organized to vary with the more detailed building activity (PBAPLUS8), and

$d$ is a scheduling diversity factor that is used here as a simplified correction for some building types were a lack of detail on the shapes of load profiles appears to cause problems.

Specific values for the unknowns $c_{s d}, P D_{\text {misc }}$, and $d$ were developed by using an iterative process and comparing them to EUI results from CEUS (CEC 2006) (see Table 2-1). The non-HVAC end use results from CEUS were selected to calibrate the plug and process peak loads, because that study appears to offer the best source of such data, and by reasoning that there is little reason the non-weather related, nonHVAC energy uses in California commercial buildings should differ from the rest of the U.S. commercial sector. The scheduling diversity factor was used for the religious worship subsectors when the squarewave load shapes were suspected to be especially inadequate.

The calibration process was carried out in five steps:

Step 1 involved assuming values for $\boldsymbol{c}_{\boldsymbol{s} d}$ and $P D_{\text {misc }}$ based on expert opinion.

Step 2 involved running the simulations using the power density model and the schedules for plug and process loads. The creation of these equipment schedules is described in section C.5 Schedules. Creating these schedules introduces Step 3.

Step 3 aggregates the results for plug and process loads by the building activities used in CEUS.

Step 4 involved comparing the results from the current modeling to those from CEUS.

Step 5 formulated new values for $c_{s d}, P D_{\text {misc }}$, and $d$.

Steps 2 through 5 were repeated eight times to obtain reasonable agreement during Step 4. Table C-25 lists the final values for $c_{s d}$ and $P D_{\text {misc }}$ used in the modeling. In general, we doubled the power density obtained from modeling the surveyed devices. 
Table C-25 Plug and Process Peak Power Levels by PBAPLUS

\begin{tabular}{|c|c|c|c|c|}
\hline $\begin{array}{l}\text { PBAPLUS8 } \\
\text { Code }\end{array}$ & PBAPLUS Name & $\mathrm{C}_{\mathrm{sd}}$ & $\begin{array}{l}P_{D_{\text {misc }}} \\
\left(W / m^{2}\right)\end{array}$ & $\begin{array}{c}\text { Schedule } \\
\text { Diversity Factor }\end{array}$ \\
\hline 1 & Vacant & 2.0 & 5.9 & 1.0 \\
\hline 2 & Administrative/professional office & 2.0 & 8.3 & 1.0 \\
\hline 3 & Bank/other financial & 2.0 & 8.3 & 1.0 \\
\hline 4 & Government office & 2.0 & 8.3 & 1.0 \\
\hline 5 & Medical office (nondiagnostic) & 2.0 & 8.3 & 1.0 \\
\hline 6 & Mixed-use office & 2.0 & 8.3 & 1.0 \\
\hline 7 & Other office & 2.0 & 8.3 & 1.0 \\
\hline 8 & Laboratory & 2.0 & 30.0 & 1.0 \\
\hline 9 & Distribution/shipping center & 2.0 & 2.0 & 1.0 \\
\hline 10 & Nonrefrigerated warehouse & 2.0 & 2.0 & 1.0 \\
\hline 11 & Self-storage & 2.0 & 2.0 & 1.0 \\
\hline 12 & Convenience store & 2.0 & 5.1 & 1.0 \\
\hline 13 & Convenience store with gas station & 2.0 & 5.1 & 1.0 \\
\hline 14 & Grocery store/food market & 2.0 & 5.1 & 1.0 \\
\hline 15 & Other food sales & 2.0 & 5.1 & 1.0 \\
\hline 16 & Fire station/police station & 2.0 & 12.0 & 1.0 \\
\hline 17 & Other public order and safety & 2.0 & 12.0 & 1.0 \\
\hline 18 & Medical office (diagnostic) & 2.0 & 5.9 & 1.0 \\
\hline 19 & Clinic/other outpatient health & 2.0 & 5.9 & 1.0 \\
\hline 20 & Refrigerated warehouse & 2.0 & 1.0 & 1.0 \\
\hline 21 & Religious worship & 2.0 & 5.9 & 0.4 \\
\hline 22 & Entertainment/culture & 2.0 & 5.9 & 1.0 \\
\hline 23 & Library & 2.0 & 5.9 & 1.0 \\
\hline 24 & Recreation & 2.0 & 5.9 & 1.0 \\
\hline 25 & Social/meeting & 2.0 & 5.9 & 1.0 \\
\hline 26 & Other public assembly & 2.0 & 5.9 & 1.0 \\
\hline 27 & College/university & 1.6 & 8.0 & 1.0 \\
\hline 28 & Elementary/middle school & 2.0 & 1.6 & 1.0 \\
\hline 29 & High school & 2.0 & 1.6 & 1.0 \\
\hline 30 & Preschool/daycare & 2.0 & 1.6 & 1.0 \\
\hline 31 & Other classroom education & 2.0 & 2.0 & 1.0 \\
\hline 32 & Fast food & 2.0 & 10.0 & 1.0 \\
\hline 33 & Restaurant/cafeteria & 2.0 & 10.0 & 1.0 \\
\hline 34 & Other food service & 2.0 & 10.0 & 1.0 \\
\hline 35 & Hospital/inpatient health & 2.0 & 8.2 & 1.0 \\
\hline 36 & Nursing home/assisted living & 2.0 & 12.0 & 1.0 \\
\hline 37 & Dormitory/fraternity/sorority & 2.0 & 3.2 & 1.0 \\
\hline 38 & Hotel & 2.0 & 3.2 & 1.0 \\
\hline 39 & Motel or inn & 2.0 & 3.2 & 1.0 \\
\hline 40 & Other lodging & 2.0 & 3.2 & 1.0 \\
\hline 41 & Vehicle dealership/showroom & 2.0 & 3.7 & 1.0 \\
\hline 42 & Retail store & 2.0 & 3.7 & 1.0 \\
\hline 43 & Other retail & 2.0 & 3.7 & 1.0 \\
\hline 44 & Post office/postal center & 2.0 & 5.9 & 1.0 \\
\hline
\end{tabular}




\begin{tabular}{|c|c|c|c|c|}
\hline $\begin{array}{l}\text { PBAPLUS8 } \\
\text { Code }\end{array}$ & PBAPLUS Name & $\mathrm{C}_{\text {sd }}$ & $\begin{array}{l}P_{\text {misc }} \\
\left(W / \mathrm{m}^{2}\right)\end{array}$ & $\begin{array}{c}\text { Schedule } \\
\text { Diversity Factor }\end{array}$ \\
\hline 45 & Repair shop & 2.0 & 5.9 & 1.0 \\
\hline 46 & Vehicle service/repair shop & 2.0 & 5.9 & 1.0 \\
\hline 47 & Vehicle storage/maintenance & 2.0 & 5.9 & 1.0 \\
\hline 48 & Other service & 2.0 & 5.9 & 1.0 \\
\hline 49 & Other & 2.0 & 5.9 & 1.0 \\
\hline
\end{tabular}

\section{C.15 Lighting Intensity}

DOE has studied the national lighting stock. Navigant (DOE 2002) used a variety of data sources, including 1999 CBECS and the XenCAPTM database, to produce national estimates for lighting stock and energy consumption. Because the goal of this study was to produce national estimates, the published data do not appear to be well-suited to producing estimates for individual CBECS buildings. The Navigant results for lighting power densities (LPDs) by PBA (from Table 5-11 in DOE 2002) are used as the mean values for a normal probability distribution and are listed in Table C-26. The standard deviations used for Gaussian assignments were arbitrarily assumed to be $1 / 15$ the magnitude of the LPD. This is a simplification, as the distribution is likely to be multi-modal, reflecting different lighting technologies (e.g., incandescent versus fluorescent). The high value for vacant buildings is likely an error in the literature data (DOE 2002). Schedule diversity factors listed in Table C-27 were developed by iteration to obtain better agreement between the models and Navigant's data for lighting energy use by attempting to correct for differences in schedules.

Table C-26 Interior LPD Assignments

\begin{tabular}{|c|l|c|c|c|c|}
\hline $\begin{array}{c}\text { PBA } \\
\text { Code }\end{array}$ & \multicolumn{1}{|c|}{ PBA Name } & $\begin{array}{c}\text { Mean } \\
\text { LPD } \\
\left(\mathbf{W} / \mathbf{f t}^{2}\right)\end{array}$ & $\begin{array}{c}\text { Standard } \\
\text { Deviation } \\
\left(\mathbf{W} / \mathbf{f t}^{2}\right)\end{array}$ & $\begin{array}{c}\text { Mean LPD } \\
\left(\mathbf{W} / \mathbf{m}^{2} \mathbf{)}\right.\end{array}$ & $\begin{array}{c}\text { Standard } \\
\text { Deviation } \\
\left(\mathbf{W} / \mathbf{m}^{2}\right)\end{array}$ \\
\hline \hline 1 & Vacant & 2.1 & 0.14 & 22.6 & 1.51 \\
\hline 2 & Office/professional & 1.8 & 0.12 & 19.4 & 1.29 \\
\hline 4 & Laboratory & 1.7 & 0.11 & 18.3 & 1.22 \\
\hline 5 & Nonrefrigerated warehouse & 1.4 & 0.09 & 15.1 & 1.01 \\
\hline 6 & Food sales & 1.9 & 0.13 & 20.5 & 1.37 \\
\hline 7 & Public order and safety & 1.3 & 0.09 & 14.0 & 0.93 \\
\hline 8 & Outpatient health care & 1.7 & 0.11 & 18.3 & 1.22 \\
\hline 11 & Refrigerated warehouse & 1.4 & 0.09 & 15.1 & 1.01 \\
\hline 12 & Religious worship & 1.4 & 0.09 & 15.1 & 1.01 \\
\hline 13 & Public assembly & 1.4 & 0.09 & 15.1 & 1.01 \\
\hline 14 & Education & 1.8 & 0.12 & 19.4 & 1.29 \\
\hline 15 & Food service & 1.6 & 0.11 & 17.2 & 1.15 \\
\hline 16 & Inpatient health care & 1.7 & 0.11 & 18.3 & 1.22 \\
\hline 17 & Skilled nursing & 1.3 & 0.09 & 14.0 & 0.93 \\
\hline 18 & Lodging & 1.3 & 0.09 & 14.0 & 0.93 \\
\hline 25 & Retail (except malls) & 1.9 & 0.13 & 20.5 & 1.37 \\
\hline 26 & Service (except food) & 1.7 & 0.11 & 18.3 & 1.22 \\
\hline 91 & Other & 1.7 & 0.11 & 18.3 & 1.22 \\
\hline
\end{tabular}


Table C-27 Interior Lighting Schedule Diversity Factors

\begin{tabular}{|c|c|c|}
\hline $\begin{array}{l}\text { PBAPLUS8 } \\
\text { Code }\end{array}$ & PBAPLUS Name & $\begin{array}{c}\text { Schedule Diversity } \\
\text { Factor }\end{array}$ \\
\hline 1 & Vacant & 1.0 \\
\hline 2 & Administrative/professional office & 0.923 \\
\hline 3 & Bank/other financial & 0.923 \\
\hline 4 & Government office & 0.923 \\
\hline 5 & Medical office (nondiagnostic) & 0.923 \\
\hline 6 & Mixed-use office & 0.923 \\
\hline 7 & Other office & 0.923 \\
\hline 8 & Laboratory & 0.695 \\
\hline 9 & Distribution/shipping center & 1.0 \\
\hline 10 & Nonrefrigerated warehouse & 1.0 \\
\hline 11 & Self-storage & 1.0 \\
\hline 12 & Convenience store & 0.876 \\
\hline 13 & Convenience store with gas station & 0.876 \\
\hline 14 & Grocery store/food market & 0.876 \\
\hline 15 & Other food sales & 0.876 \\
\hline 16 & Fire station/police station & 0.627 \\
\hline 17 & Other public order and safety & 0.627 \\
\hline 18 & Medical office (diagnostic) & 0.962 \\
\hline 19 & Clinic/other outpatient health & 0.962 \\
\hline 20 & Refrigerated warehouse & 0.573 \\
\hline 21 & Religious worship & 0.972 \\
\hline 22 & Entertainment/culture & 0.779 \\
\hline 23 & Library & 0.779 \\
\hline 24 & Recreation & 0.779 \\
\hline 25 & Social/meeting & 0.779 \\
\hline 26 & Other public assembly & 0.779 \\
\hline 27 & College/university & 0.773 \\
\hline 28 & Elementary/middle school & 0.773 \\
\hline 29 & High school & 0.773 \\
\hline 30 & Preschool/daycare & 0.773 \\
\hline 31 & Other classroom education & 0.773 \\
\hline 32 & Fast food & 1.0 \\
\hline 33 & Restaurant/cafeteria & 1.0 \\
\hline 34 & Other food service & 1.0 \\
\hline 35 & Hospital/inpatient health & 0.680 \\
\hline 36 & Nursing home/assisted living & 0.529 \\
\hline 37 & Dormitory/fraternity/sorority & 0.565 \\
\hline 38 & Hotel & 0.565 \\
\hline 39 & Motel or inn & 0.565 \\
\hline 40 & Other lodging & 0.565 \\
\hline 41 & Vehicle dealership/showroom & 0.963 \\
\hline 42 & Retail store & 0.963 \\
\hline 43 & Other retail & 0.963 \\
\hline 44 & Post office/postal center & 0.963 \\
\hline
\end{tabular}




\begin{tabular}{|c|l|c|}
\hline $\begin{array}{c}\text { PBAPLUS8 } \\
\text { Code }\end{array}$ & PBAPLUS Name & $\begin{array}{c}\text { Schedule Diversity } \\
\text { Factor }\end{array}$ \\
\hline \hline 45 & Repair shop & 0.943 \\
\hline 46 & Vehicle service/repair shop & 0.943 \\
\hline 47 & Vehicle storage/maintenance & 0.943 \\
\hline 48 & Other service & 0.943 \\
\hline 49 & Other & 0.839 \\
\hline
\end{tabular}

\section{C.16 Infiltration Rate}

Very few data are available for determining appropriate infiltration rates to use in modeling. Persily (1998) reviewed literature with measured data about a combined total of 139 commercial buildings and concluded that commercial buildings are not tighter than residential buildings. Although the data set is minimal and not formulated from a suitable random sample, he also concluded that there is little evidence to support the notion that age and type of construction affect airtightness.

In this study, infiltration rates are modeled using a simple method based on constant air change rates over time. Annual average air change rates are modeled by combining a flow rate per exterior area with a whole-zone air change rate.

The bulk of the infiltration is attributed to leaks in the thermal envelope. However, infiltration rates for commercial buildings are characterized at relatively high pressure differences of $75 \mathrm{~Pa}$. In this study, we generated flow rate per area values for annual average conditions by scaling values for $75 \mathrm{~Pa}$ to the flow

for 4 Pa using the usual relationship $\quad \frac{\dot{V}}{A}=C \Delta P^{n}$ using a flow exponent $n=0.65$.

For the assignments in this study, we assumed a leakage rate of $0.4\left(\mathrm{cfm} / \mathrm{ft}^{2}\right)$ at $75 \mathrm{~Pa}$. This value is a factor of 4 lower than the mean value of $1.67\left(\mathrm{cfm} / \mathrm{ft}^{2}\right)$ at $75 \mathrm{~Pa}$ reported by Persily (1998), but is used here as a continuous, annual average leakage rate for a constant infiltration model (rather than a flow that will actually vary over the course of the year). This value was scaled to a rate of $0.0002677 \mathrm{~m}^{3} / \mathrm{s} / \mathrm{m}^{2}$ at 4 $\mathrm{Pa}$. The preprocessor used to generate the individual EnergyPlus input files uses this value with a calculation of the exterior envelope area and produces an annual average air change rate for each zone.

The whole-zone air change is applied to all zones regardless of their proximity to the envelope. The magnitude of the whole zone component was assigned, by assumption, by taking $10 \%$ of a randomly assigned level determined using a lognormal probability distribution for infiltration found by Chan (2006). Chan's modeling study found infiltration rates in the commercial sector to have a lognormal distribution with geometric mean of 0.35 and a geometric standard deviation of 2.1 (see Figure 5-22 in Chan 2006).

Once the set of buildings has been simulated, we can compare the distribution of air change rates in the models to distribution obtained by Chan. This comparison is provided in Figure 4-14.

\section{C.17 Exterior Lighting}

An exterior lighting load is assigned for illuminating the building's facade based on Table 9.4.5 in ASHRAE 2004a. The entire perimeter of the building at ground level is illuminated at the minimum power density of $0.5 \mathrm{~W}$ per linear foot $(1.64 \mathrm{~W}$ per linear meter). No parking lot or garage lighting is included in the analysis. This tends to yield relatively low values for exterior lighting loads because many existing buildings have much less efficient lighting than that specified in Standard 90.1-2004. These assignments need to be improved and the Navigant study (DOE 2002) may be useful. 


\section{C.18 HVAC Systems}

The HVAC systems are modeled in EnergyPlus by using system types that are available for modeling in the EnergyPlus-based framework and attempting to follow as many of the CBECS variables as is practical. The first step is to determine if the building has heating and/or cooling using :

- Percent cooled (COOLP8)

- Percent heated (HEATP8).

Figure C-3 shows the distribution of CBECS data for percent cooled and heated. The most common response is $100 \%$ of the building cooled and heated. The next most common response is $0 \%$ of the building heated or cooled. For this study, we made the important simplification that buildings are either completely conditioned or not conditioned at all; the determination is made separately for heating and cooling. This simplification is very helpful for the following reasons:

- There is no way to determine which portion of a building is or is not conditioned.

- The preprocessor cannot yet generate EnergyPlus models where only some of the zones are conditioned.

We assumed that buildings that are more than $25 \%$ conditioned (COOLP8 $>25$; HEATP $8>25$ ) were entirely conditioned, and those with $25 \%$ or less were not conditioned. The $25 \%$ level was chosen arbitrarily after inspecting the data in Figure C-3. The models can also be only cooled or only heated.

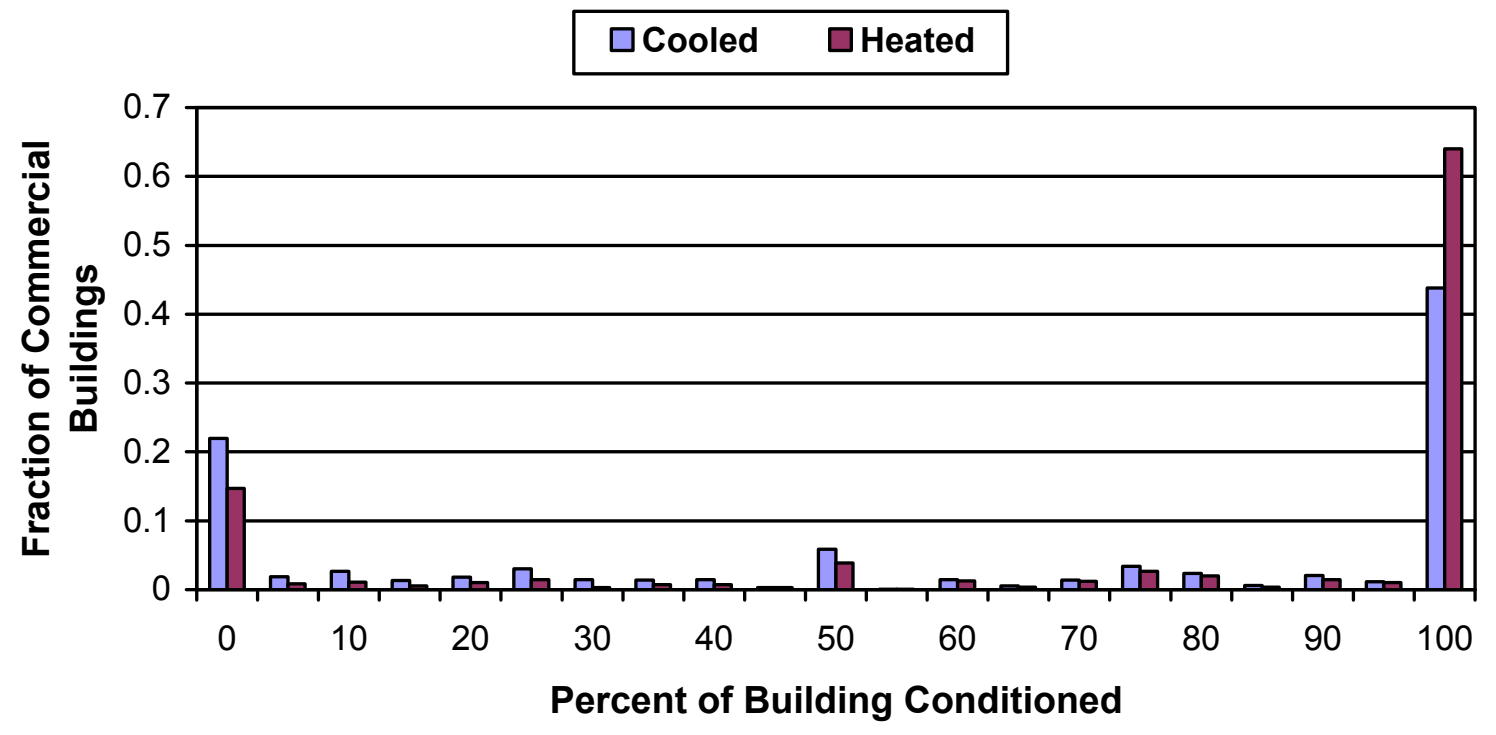

Figure C-3 Analysis of Percent Cooled and Heated Data (PDF for COOLP8 and HEATP8)

The next important variables used to assign a type of HVAC system to the model are the main cooling type (MAINCL8) and the main heating type (MAINHT8). Figure C-4 shows the joint probability distribution for these two variables. Table C-28 shows a matrix of the number of buildings in 2003 CBECS data set with the various combinations of values for MAINCL8 and MAINHT8. Table C-29 shows similar data but with the filtering applied that screens out if the percentage of conditioned floor is less than $25 \%$. 


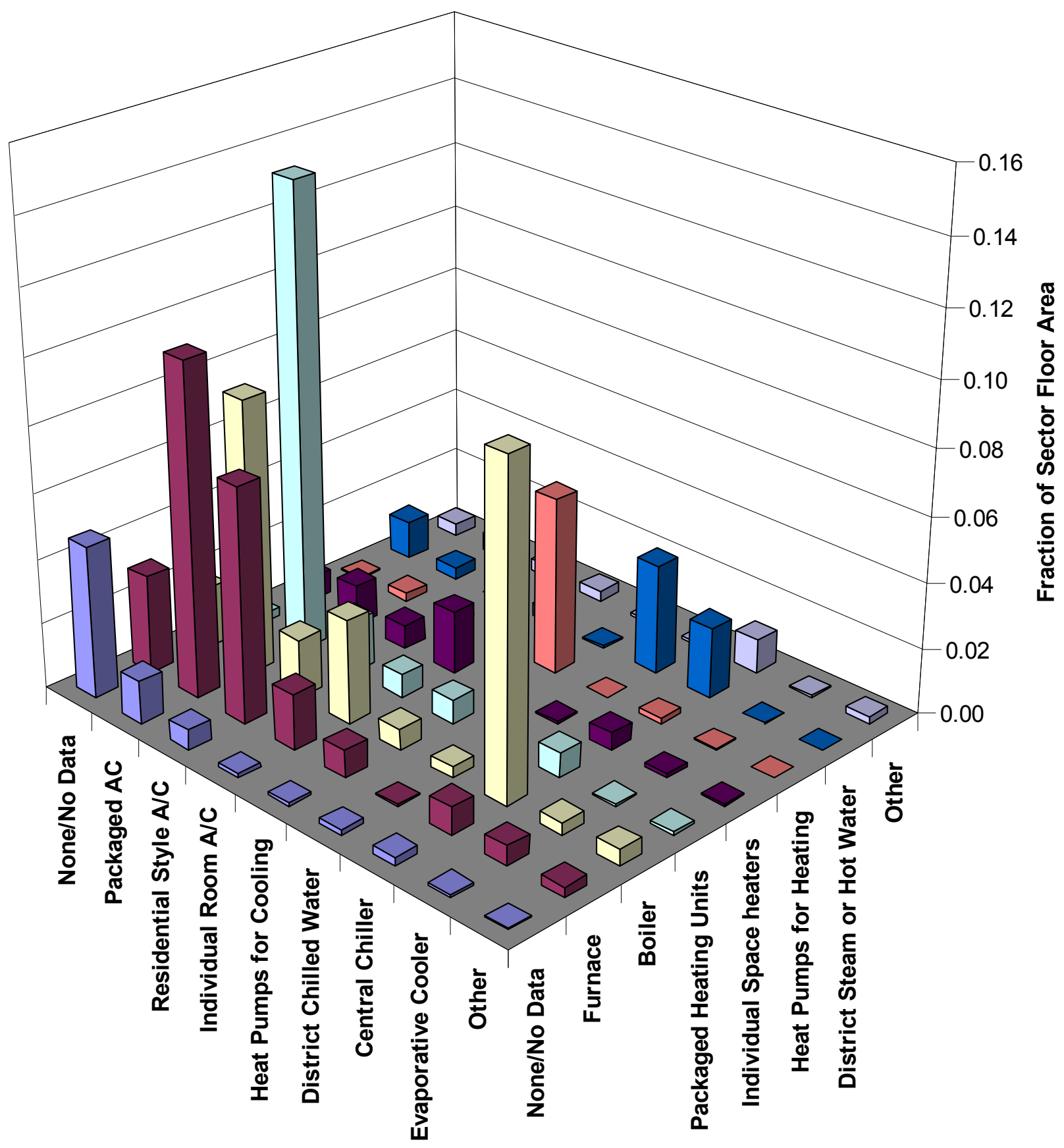

Figure C-4 Joint Discrete PDF for MAINCL8 and MAINHT8 (Raw; All Buildings Regardless of Percent Conditioned) 
Table C-28 Number of Buildings for Each Combination of MAINCL8 and MAINHT8 (Raw Data, All)

\begin{tabular}{|l|c|c|c|c|c|c|c|c|}
\hline & None & Furnace & Boiler & $\begin{array}{c}\text { Packaged } \\
\text { Heating } \\
\text { Units }\end{array}$ & $\begin{array}{c}\text { Individual } \\
\text { Space } \\
\text { Heaters }\end{array}$ & $\begin{array}{c}\text { Heat } \\
\text { Pumps } \\
\text { for } \\
\text { Heating }\end{array}$ & $\begin{array}{c}\text { District } \\
\text { Steam } \\
\text { or Hot } \\
\text { Water }\end{array}$ & Other \\
\hline None & 233 & 149 & 92 & 19 & 44 & 3 & 18 & 25 \\
\hline Packaged A/C & 59 & 538 & 328 & 727 & 58 & 14 & 17 & 27 \\
\hline $\begin{array}{l}\text { Residential style } \\
\text { A/C }\end{array}$ & 29 & 412 & 84 & 100 & 36 & 4 & 3 & 13 \\
\hline $\begin{array}{l}\text { Individual room } \\
\text { A/C }\end{array}$ & 11 & 97 & 141 & 29 & 126 & 13 & 13 & 20 \\
\hline $\begin{array}{l}\text { Heat pumps for } \\
\text { cooling }\end{array}$ & 7 & 35 & 22 & 20 & 4 & 317 & 2 & 6 \\
\hline $\begin{array}{l}\text { District chilled } \\
\text { water }\end{array}$ & 4 & 4 & 14 & 7 & 2 & 0 & 145 & 3 \\
\hline Central chillers & 13 & 23 & 434 & 26 & 14 & 6 & 85 & 36 \\
\hline $\begin{array}{l}\text { Evaporative } \\
\text { coolers }\end{array}$ & 5 & 30 & 17 & 5 & 6 & 2 & 1 & 4 \\
\hline Other & 1 & 7 & 13 & 4 & 2 & 0 & 1 & 11 \\
\hline
\end{tabular}

Table C-29 Number of Buildings for Each Combination of MAINCL8 and MAINHT8 (Filtered for COOLP8 and HEATP8>25\%)

\begin{tabular}{|l|c|c|c|c|c|c|c|c|}
\hline & $\begin{array}{c}\text { None } \\
\text { (and } \\
\text { HEATP8<25) }\end{array}$ & Furnace & Boiler & $\begin{array}{c}\text { Packaged } \\
\text { Heating } \\
\text { Units }\end{array}$ & $\begin{array}{c}\text { Individual } \\
\text { Space } \\
\text { Heaters }\end{array}$ & $\begin{array}{c}\text { Heat } \\
\text { Pumps } \\
\text { for } \\
\text { Heating }\end{array}$ & $\begin{array}{c}\text { District } \\
\text { Steam } \\
\text { or Hot } \\
\text { Water }\end{array}$ & Other \\
\hline $\begin{array}{l}\text { None (and } \\
\text { COOLP8<25) }\end{array}$ & 0 & 136 & 88 & 18 & 29 & 3 & 18 & 22 \\
\hline $\begin{array}{l}\text { Packaged } \\
\text { A/C }\end{array}$ & 44 & 458 & 263 & 664 & 32 & 12 & 13 & 21 \\
\hline $\begin{array}{l}\text { Residential } \\
\text { style A/C }\end{array}$ & 23 & 351 & 73 & 78 & 27 & 4 & 3 & 8 \\
\hline $\begin{array}{l}\text { Individual } \\
\text { room A/C }\end{array}$ & 8 & 57 & 73 & 20 & 99 & 13 & 9 & 12 \\
\hline $\begin{array}{l}\text { Heat pumps } \\
\text { for cooling }\end{array}$ & 7 & 26 & 20 & 15 & 1 & 300 & 1 & 3 \\
\hline $\begin{array}{l}\text { District } \\
\text { chilled water }\end{array}$ & 12 & 4 & 13 & 7 & 2 & 0 & 145 & 3 \\
\hline $\begin{array}{l}\text { Central } \\
\text { chillers }\end{array}$ & 4 & 22 & 431 & 23 & 14 & 5 & 83 & 36 \\
\hline $\begin{array}{l}\text { Evaporative } \\
\text { coolers }\end{array}$ & 1 & 28 & 12 & 5 & 3 & 2 & 1 & 3 \\
\hline Other & 0 & 6 & 12 & 3 & 0 & 0 & 1 & 9 \\
\hline
\end{tabular}


Each combination of possible values for MAINCL8 and MAINHT8 are assigned a HVAC system type. Table C-30 shows the mappings from MAINCL8 and MAINHT8 to a HVAC system type identified by number. The numbers shown in Table C-30 correspond to the HVAC systems listed in Table C-31. Fifty-two system topologies were identified and implemented for analysis.

Table C-30 Mappings from MAINCL8 and MAINHT8 to Number of HVAC System as Modeled (Listed in Table C-31)

\begin{tabular}{|l|c|c|c|c|c|c|c|c|}
\hline & None & Furnace & Boiler & $\begin{array}{c}\text { Packaged } \\
\text { Heating } \\
\text { Units }\end{array}$ & $\begin{array}{c}\text { Individual } \\
\text { Space } \\
\text { Heaters }\end{array}$ & $\begin{array}{c}\text { Heat } \\
\text { Pumps } \\
\text { for } \\
\text { Heating }\end{array}$ & $\begin{array}{c}\text { District } \\
\text { Steam } \\
\text { or Hot } \\
\text { Water }\end{array}$ & Other \\
\hline None & 0 & 19 & 29 & 30 & 31 & 32 & 37 & 21 \\
\hline Packaged A/C & 24 & 3 & 5 & 3 & 22 & 4 & 38 & 6 \\
\hline $\begin{array}{l}\text { Residential style } \\
\text { A/C }\end{array}$ & 21 & 9 & 16 & 9 & 23 & 10 & 17 & 9 \\
\hline $\begin{array}{l}\text { Individual room } \\
\text { A/C }\end{array}$ & 15 & 11 & 12 & 13 & 14 & 4 & 18 & 14 \\
\hline $\begin{array}{l}\text { Heat pumps for } \\
\text { cooling }\end{array}$ & 35 & 33 & $1 *$ & 33 & 34 & $2 *$ & 36 & $2^{*}$ \\
\hline $\begin{array}{l}\text { District chilled } \\
\text { water }\end{array}$ & 34 & 20 & 40 & 41 & 42 & 0 & 43 & 39 \\
\hline Central chillers & 45 & 25 & 7 & 26 & 27 & 28 & 44 & 8 \\
\hline $\begin{array}{l}\text { Evaporative } \\
\text { coolers }\end{array}$ & 46 & 47 & 48 & 49 & 50 & 51 & 52 & 47 \\
\hline Other & 24 & 3 & 7 & 3 & 14 & 2 & 43 & 7 \\
\hline
\end{tabular}

${ }^{*}$ Modeled as air source heat pumps

Table C-31 Modeled HVAC System Configurations with Mappings to CBECS Variables

\begin{tabular}{|c|l|l|l|c|c|}
\hline $\begin{array}{c}\text { Sys } \\
\text { No. }\end{array}$ & \multicolumn{1}{|c|}{ Name } & Distribution & Main Components & $\begin{array}{c}\text { Mech. } \\
\text { Vent }\end{array}$ & $\begin{array}{c}\text { CBECS Variable Sets } \\
\text { (MAINHT8, MAINCL8) }\end{array}$ \\
\hline 1 & $\begin{array}{l}\text { PTAC air } \\
\text { source }\end{array}$ & $\begin{array}{l}\text { Multi-zone } \\
\text { hydronic loop, } \\
\text { zone PTAC }\end{array}$ & $\begin{array}{l}\text { Boiler, air-to-air DX } \\
\text { cooling, hot water coil }\end{array}$ & yes & $(2,4)$ \\
\hline 2 & $\begin{array}{l}\text { PTHP air } \\
\text { source }\end{array}$ & Single-zone air & $\begin{array}{l}\text { Water to air or air to } \\
\text { air coil heat/cool }\end{array}$ & yes & $(5,4),(5,8)$ \\
\hline 3 & PSZ-AC & Single-zone air & $\begin{array}{l}\text { DX coil, gas coil, const } \\
\text { fan }\end{array}$ & yes & $(1,1),(3,1),(1,8),(3,8)$ \\
\hline 4 & PSZ-HP & Single-zone air & $\begin{array}{l}\text { DX coil cool/heat, } \\
\text { const fan }\end{array}$ & yes & $(5,1),(5,3)$ \\
\hline 5 & $\begin{array}{l}\text { Packaged } \\
\text { VAV w/reheat }\end{array}$ & Multi-zone air & $\begin{array}{l}\text { DX coil, VAV fan, } \\
\text { boiler, hot water VAV } \\
\text { boxes }\end{array}$ & yes & $(2,1)$ \\
\hline 6 & $\begin{array}{l}\text { Packaged } \\
\text { VAV w/PFP } \\
\text { boxes }\end{array}$ & Multi-zone air & $\begin{array}{l}\text { DX coil, VAV fan, fan- } \\
\text { powered induction } \\
\text { boxes, electric reheat }\end{array}$ & yes & $(7,1)$ \\
\hline
\end{tabular}




\begin{tabular}{|c|c|c|c|c|c|}
\hline $\begin{array}{l}\text { Sys } \\
\text { No. }\end{array}$ & Name & Distribution & Main Components & $\begin{array}{l}\text { Mech. } \\
\text { Vent }\end{array}$ & $\begin{array}{l}\text { CBECS Variable Sets } \\
\text { (MAINHT8, MAINCL8) }\end{array}$ \\
\hline 7 & $\begin{array}{l}\text { VAV } \\
\text { w/Reheat }\end{array}$ & Multi-zone air & $\begin{array}{l}\text { AHU, CW coil, hot } \\
\text { water coil, VAV fan, } \\
\text { chiller, boiler, hot } \\
\text { water VAV boxes }\end{array}$ & yes & $(2,6),(2,8),(7,8)$ \\
\hline 8 & $\begin{array}{l}\text { VAV w/PFP } \\
\text { boxes }\end{array}$ & Multi-zone air & $\begin{array}{l}\text { AHU, CW coil, hot } \\
\text { water coil, VAV fan, } \\
\text { chiller, fan-powered } \\
\text { induction boxes, } \\
\text { electric reheat }\end{array}$ & yes & $(7,6)$ \\
\hline 9 & $\begin{array}{l}\text { Residential } \\
\text { forced air }\end{array}$ & Single-zone air & $\begin{array}{l}\text { DX coil, gas coil, const } \\
\text { fan }\end{array}$ & no & $(1,2),(3,2),(7,2)$ \\
\hline 10 & $\begin{array}{l}\text { Residential } \\
\text { heat pump }\end{array}$ & Single-zone air & $\begin{array}{l}\text { DX coil heat/cool, } \\
\text { const fan }\end{array}$ & no & $(5,2)$ \\
\hline 11 & $\begin{array}{l}\text { Window A/C } \\
\text { w/forced air } \\
\text { furnace }\end{array}$ & $\begin{array}{l}\text { Single-zone air, } \\
\text { zone cool }\end{array}$ & $\begin{array}{l}\text { Gas coil, const fan; } \\
\text { zone window A/C }\end{array}$ & no & $(1,3)$ \\
\hline 12 & $\begin{array}{l}\text { Window A/C } \\
\text { w/hot water } \\
\text { baseboards }\end{array}$ & $\begin{array}{l}\text { Multi-zone } \\
\text { hydronic heat; } \\
\text { zone cool } \\
\end{array}$ & $\begin{array}{l}\text { Boiler, pump, hot } \\
\text { water baseboards, } \\
\text { window A/C }\end{array}$ & no & $(2,3)$ \\
\hline 13 & $\begin{array}{l}\text { Window } A / C ; \\
\text { unit heater }\end{array}$ & $\begin{array}{l}\text { Zone cool, zone } \\
\text { heat }\end{array}$ & $\begin{array}{l}\text { Window } A / C \text {, unit } \\
\text { heater }\end{array}$ & no & $(3,3)$ \\
\hline 14 & $\begin{array}{l}\text { Window A/C; } \\
\text { electric } \\
\text { baseboards }\end{array}$ & $\begin{array}{l}\text { Zone cool, zone } \\
\text { heat }\end{array}$ & $\begin{array}{l}\text { Window } A / C \text {, electric } \\
\text { baseboards }\end{array}$ & no & $(4,3),(7,3),(4,8)$ \\
\hline 15 & Window $\mathrm{A} / \mathrm{C}$ & Zone cool & Window AC & no & $(0,3)$ \\
\hline 16 & $\begin{array}{l}\text { Residential } \\
\text { forced air } \\
\text { cooling, hot } \\
\text { water } \\
\text { baseboards }\end{array}$ & $\begin{array}{l}\text { Single-zone } \\
\text { cool, multizone } \\
\text { hydronic heat }\end{array}$ & $\begin{array}{l}\text { DX coil, const fan, } \\
\text { boiler, pump, hot } \\
\text { water baseboards }\end{array}$ & no & $(2,2)$ \\
\hline 17 & $\begin{array}{l}\text { Residential } \\
\text { forced air } \\
\text { w/district hot } \\
\text { water }\end{array}$ & $\begin{array}{l}\text { Single-zone air; } \\
\text { multi-zone } \\
\text { hydronic loop }\end{array}$ & $\begin{array}{l}\text { DX coil, const fan; } \\
\text { purchased hot water, } \\
\text { hot water baseboards }\end{array}$ & no & $(6,2)$ \\
\hline 18 & $\begin{array}{l}\text { Window A/C } \\
\text { w/district hot } \\
\text { water }\end{array}$ & $\begin{array}{l}\text { Zone cool, multi- } \\
\text { zone hydronic } \\
\text { loop }\end{array}$ & $\begin{array}{l}\text { Window } \mathrm{A} / \mathrm{C} \text {, } \\
\text { purchased hot water, } \\
\text { hot water baseboards }\end{array}$ & no & $(6,3)$ \\
\hline 19 & $\begin{array}{l}\text { Forced air } \\
\text { furnace }\end{array}$ & Single-zone air & Gas coil, const fan & yes & $(1,0)$ \\
\hline 20 & $\begin{array}{l}\text { Forced air } \\
\text { furnace, } \\
\text { district chilled } \\
\text { water fan coil }\end{array}$ & $\begin{array}{l}\text { Single-zone air; } \\
\text { mult-izone } \\
\text { hydronic chilled } \\
\text { water }\end{array}$ & $\begin{array}{l}\text { Unit heater/ventilator } \\
\text { with gas coil; 4-pipe } \\
\text { fan coils; purchased } \\
\text { chilled water }\end{array}$ & yes & $(1,5)$ \\
\hline 21 & $\begin{array}{l}\text { Residential } \\
\text { A/C (no heat) }\end{array}$ & Single-zone air & DX coil, const fan & no & $(0,2)$ \\
\hline 22 & $\begin{array}{l}\text { PSZ-A/C } \\
\text { w/electric } \\
\text { baseboards }\end{array}$ & $\begin{array}{l}\text { Single-zone air; } \\
\text { zone heat }\end{array}$ & $\begin{array}{l}\text { DX coil, const fan; } \\
\text { zone electric } \\
\text { baseboards }\end{array}$ & yes & $(4,1)$ \\
\hline 23 & $\begin{array}{l}\text { Residential } \\
\mathrm{A} / \mathrm{C} \text { w/electric } \\
\text { baseboards }\end{array}$ & $\begin{array}{l}\text { Single-zone air; } \\
\text { zone heat }\end{array}$ & $\begin{array}{l}\text { DX coil, const fan; } \\
\text { zone electric } \\
\text { baseboards }\end{array}$ & no & $(4,2)$ \\
\hline
\end{tabular}




\begin{tabular}{|c|c|c|c|c|c|}
\hline $\begin{array}{l}\text { Sys } \\
\text { No. }\end{array}$ & Name & Distribution & Main Components & $\begin{array}{l}\text { Mech. } \\
\text { Vent }\end{array}$ & $\begin{array}{l}\text { CBECS Variable Sets } \\
\text { (MAINHT8, MAINCL8) }\end{array}$ \\
\hline 24 & $\begin{array}{l}\text { PSZ-AC (no } \\
\text { heat) }\end{array}$ & Single-zone air & DX coil, const fan & yes & $(0,1),(1,8)$ \\
\hline 25 & $\begin{array}{l}\text { VAV w/gas } \\
\text { reheat }\end{array}$ & Multi-zone air & $\begin{array}{l}\text { AHU, cold water coil, } \\
\text { gas coil, VAV fan, } \\
\text { chiller, }\end{array}$ & yes & $(1,6)$ \\
\hline 26 & $\begin{array}{l}\text { VAV w/zone } \\
\text { unit heaters }\end{array}$ & $\begin{array}{l}\text { Multi-zone air, } \\
\text { zone heat }\end{array}$ & $\begin{array}{l}\text { AHU, cold water coil, } \\
\text { gas coil, VAV fan, } \\
\text { chiller, single duct, } \\
\text { zone unit heater }\end{array}$ & yes & $(3,6)$ \\
\hline 27 & $\begin{array}{l}\text { VAV } \\
\text { w/electric } \\
\text { baseboards }\end{array}$ & $\begin{array}{l}\text { Multi-zone air, } \\
\text { zone heat }\end{array}$ & $\begin{array}{l}\text { AHU, cold water coil, } \\
\text { VAV fan, chiller, single } \\
\text { duct, electric } \\
\text { baseboards }\end{array}$ & yes & $(4,6)$ \\
\hline 28 & $\begin{array}{l}\text { VAV cool } \\
\text { with zone } \\
\text { heat pump } \\
\text { heating }\end{array}$ & $\begin{array}{l}\text { Multi-zone air; } \\
\text { zone heat }\end{array}$ & $\begin{array}{l}\text { AHU, cold water coil, } \\
\text { VAV fan, chiller, single } \\
\text { duct, packaged hep }\end{array}$ & yes & $(5,6)$ \\
\hline 29 & $\begin{array}{l}\text { Hot water } \\
\text { baseboards }\end{array}$ & $\begin{array}{l}\text { Multi-zone } \\
\text { hydronic heat }\end{array}$ & $\begin{array}{l}\text { Boiler pump, hot water } \\
\text { baseboards }\end{array}$ & no & $(2,0)$ \\
\hline 30 & Unit heaters & Zone heat & Unit heaters & no & $(3,0)$ \\
\hline 31 & $\begin{array}{l}\text { Electric } \\
\text { baseboards }\end{array}$ & Zone heat & Electric baseboards & no & $(4,0)$ \\
\hline 32 & $\begin{array}{l}\text { Heat pump } \\
\text { heating }\end{array}$ & Single-zone air & $\begin{array}{l}\text { DX coil heating, const } \\
\text { fan }\end{array}$ & yes & $(5,0)$ \\
\hline 33 & $\begin{array}{l}\text { PTAC with } \\
\text { gas coil heat }\end{array}$ & Single-zone air & $\begin{array}{l}\text { Air-to-air cool, gas coil } \\
\text { heat }\end{array}$ & yes & $(1,4),(3,4)$ \\
\hline 34 & $\begin{array}{l}\text { PTAC } \\
\text { w/electric } \\
\text { baseboards }\end{array}$ & $\begin{array}{l}\text { Single-zone air, } \\
\text { zone heat }\end{array}$ & $\begin{array}{l}\text { Air-to-air cool, electric } \\
\text { baseboards }\end{array}$ & yes & $(4,4)$ \\
\hline 35 & $\begin{array}{l}\text { PTAC (no } \\
\text { heat) }\end{array}$ & Single-zone air & Air-to-air cool & yes & $(0,4)$ \\
\hline 36 & $\begin{array}{l}\text { PTAC } \\
\text { w/district hot } \\
\text { water }\end{array}$ & $\begin{array}{l}\text { Multi-zone } \\
\text { hydronic loop, } \\
\text { single zone air, } \\
\text { zone heat }\end{array}$ & $\begin{array}{l}\text { Air-to-air cool, } \\
\text { purchased hot water, } \\
\text { hot water baseboard }\end{array}$ & yes & $(6,4)$ \\
\hline 37 & $\begin{array}{l}\text { District hot } \\
\text { water } \\
\text { baseboards }\end{array}$ & $\begin{array}{l}\text { Multizone } \\
\text { hydronic loop }\end{array}$ & $\begin{array}{l}\text { Purchased hot water, } \\
\text { hot water baseboards }\end{array}$ & no & $(6,0)$ \\
\hline 38 & $\begin{array}{l}\text { PSZ-AC } \\
\text { w/district hot } \\
\text { water }\end{array}$ & $\begin{array}{l}\text { Single-zone air, } \\
\text { multizone } \\
\text { hydronic loop }\end{array}$ & $\begin{array}{l}\text { DX coil, const fan, } \\
\text { purchased hot water, } \\
\text { hot water baseboards }\end{array}$ & yes & $(6,1)$ \\
\hline 39 & $\begin{array}{l}\text { District } \\
\text { chilled water } \\
\text { fan coil }\end{array}$ & $\begin{array}{l}\text { Multi-zone } \\
\text { hydronic chilled } \\
\text { water }\end{array}$ & $\begin{array}{l}\text { 4-pipe fan coil; } \\
\text { purchased chilled } \\
\text { water }\end{array}$ & no & $(0,5)$ \\
\hline 40 & $\begin{array}{l}\text { Fan coil, } \\
\text { district chilled } \\
\text { water and } \\
\text { boiler }\end{array}$ & $\begin{array}{l}\text { Multi-zone } \\
\text { hydronic chilled } \\
\text { and hot water }\end{array}$ & $\begin{array}{l}\text { 4-pipe fan coil, boiler; } \\
\text { purchased chilled } \\
\text { water }\end{array}$ & no & $(2,5)$ \\
\hline 41 & $\begin{array}{l}\text { Fan coil } \\
\text { district chilled } \\
\text { water, unit } \\
\text { heaters }\end{array}$ & $\begin{array}{l}\text { Multi-zone } \\
\text { hydronic chilled } \\
\text { water }\end{array}$ & $\begin{array}{l}\text { 4-pipe fan coil cooling, } \\
\text { unit heater; purchased } \\
\text { chilled water }\end{array}$ & no & $(3,5)$ \\
\hline
\end{tabular}




\begin{tabular}{|c|c|c|c|c|c|}
\hline $\begin{array}{l}\text { Sys } \\
\text { No. }\end{array}$ & Name & Distribution & Main Components & $\begin{array}{c}\text { Mech. } \\
\text { Vent }\end{array}$ & $\begin{array}{l}\text { CBECS Variable Sets } \\
\text { (MAINHT8, MAINCL8) }\end{array}$ \\
\hline 42 & $\begin{array}{l}\text { Fan coil } \\
\text { district chilled } \\
\text { water, } \\
\text { electric } \\
\text { baseboards }\end{array}$ & $\begin{array}{l}\text { Multi-zone } \\
\text { hydronic chilled } \\
\text { water; zone heat }\end{array}$ & $\begin{array}{l}\text { 4-pipe fan coil cooling, } \\
\text { electric baseboards; } \\
\text { purchased chilled } \\
\text { water }\end{array}$ & no & $(4,5)$ \\
\hline 43 & $\begin{array}{l}\text { Fan coil, } \\
\text { district hot } \\
\text { and cold } \\
\text { water }\end{array}$ & $\begin{array}{l}\text { Multi-zone } \\
\text { hydronic }\end{array}$ & $\begin{array}{l}\text { 4-pipe fan coil, } \\
\text { purchased chilled } \\
\text { water, purchased hot } \\
\text { water }\end{array}$ & no & $(6,5),(7,5)$ \\
\hline 44 & $\begin{array}{l}\text { Fan coil, } \\
\text { District hot } \\
\text { water and } \\
\text { chiller }\end{array}$ & $\begin{array}{l}\text { Multi-zone } \\
\text { hydronic }\end{array}$ & $\begin{array}{l}\text { 4-pipe fan coil, chiller, } \\
\text { purchased hot water }\end{array}$ & no & $(6,6)$ \\
\hline 45 & $\begin{array}{l}\text { Fan coil } \\
\text { chiller }\end{array}$ & $\begin{array}{l}\text { Multi-zone } \\
\text { hydronic }\end{array}$ & 4-pipe fan coil, chiller & no & $(0,6)$ \\
\hline 46 & $\begin{array}{l}\text { Direct evap } \\
\text { cooler }\end{array}$ & Single-zone cool & Direct evap cooler & yes & $(0,7)$ \\
\hline 47 & $\begin{array}{l}\text { Forced air } \\
\text { furnace, } \\
\text { direct evap } \\
\text { cooler }\end{array}$ & Single-zone air & $\begin{array}{l}\text { Gas coil, direct evap } \\
\text { cooler }\end{array}$ & yes & $(1,7),(7,7)$ \\
\hline 48 & $\begin{array}{l}\text { Hot water } \\
\text { baseboards, } \\
\text { direct evap } \\
\text { cooler }\end{array}$ & $\begin{array}{l}\text { Single-zone air, } \\
\text { zone heat, multi- } \\
\text { zone hydronic }\end{array}$ & $\begin{array}{l}\text { Boiler, direct evap } \\
\text { cooler, hot water } \\
\text { baseboards }\end{array}$ & yes & $(2,7)$ \\
\hline 49 & $\begin{array}{l}\text { Direct evap } \\
\text { cooler, unit } \\
\text { heaters }\end{array}$ & $\begin{array}{l}\text { Zone heat, } \\
\text { single-zone air }\end{array}$ & $\begin{array}{l}\text { Direct evap cooler, } \\
\text { gas unit heater }\end{array}$ & yes & $(3,7)$ \\
\hline 50 & $\begin{array}{l}\text { Electric } \\
\text { baseboards, } \\
\text { direct evap } \\
\text { cooler }\end{array}$ & $\begin{array}{l}\text { Zone heat, } \\
\text { single-zone air }\end{array}$ & $\begin{array}{l}\text { Direct evap cooler, } \\
\text { electric baseboards }\end{array}$ & yes & $(4,7)$ \\
\hline 51 & $\begin{array}{l}\text { Heat pump } \\
\text { heating, } \\
\text { direct evap } \\
\text { cooler }\end{array}$ & Single-zone air & $\begin{array}{l}\text { DX coil heating, const } \\
\text { fan, direct evap cooler }\end{array}$ & yes & $(5,7)$ \\
\hline 52 & $\begin{array}{l}\text { District hot } \\
\text { water } \\
\text { baseboards, } \\
\text { direct evap } \\
\text { cooler }\end{array}$ & $\begin{array}{l}\text { Multi-zone } \\
\text { hydronic loop, } \\
\text { single-zone air }\end{array}$ & $\begin{array}{l}\text { Purchased hot water, } \\
\text { hot water baseboards; } \\
\text { direct evap cooler }\end{array}$ & yes & $(6,7)$ \\
\hline
\end{tabular}

Once the basic topology of the HVAC system has been determined, the next step is to determine certain details for performance levels and optional features. The CBECS variable for economizer cycle (ECN8) is available to determine whether the system has an economizer. We assume that no economizer cycle is possible if the value of ECN8 is 0. If the building has an economizer cycle but the system type mappings do not call for mechanical ventilation, an outside air system was added to the system topology. Economizer cycle (ECN8):

- 0 no data-assumed no economizer, or not operating

- 1 yes

- 2 no 
For these buildings, we assumed outside air systems have gravity dampers that would open when the equipment ran by setting the minimum outdoor air fraction to 1.0 during unoccupied hours via EnergyPlus schedules.

The components performance levels used within each system are discussed in the next section.

\section{C.19 HVAC Components}

This section discusses the performance parameters that need to be defined for each component in the HVAC system, including efficiency, coefficient of performance (COP), static pressure, and ventilation rates. In general, we would expect these to be complicated functions of the type of system and component, capacity, age, and maintenance history of the equipment. The age and maintenance situation are modeled from the results of the 2003 CBECS variables:

- HVAC equipment upgrade (RENHVC8). We assume this is already represented in the type of equipment present.

- Main cooling equipment replaced since 1990 (NWMNCL8).

- Main heating equipment replaced since 1990 (NWMNHT8).

- Regular HVAC maintenance (MAINT8).

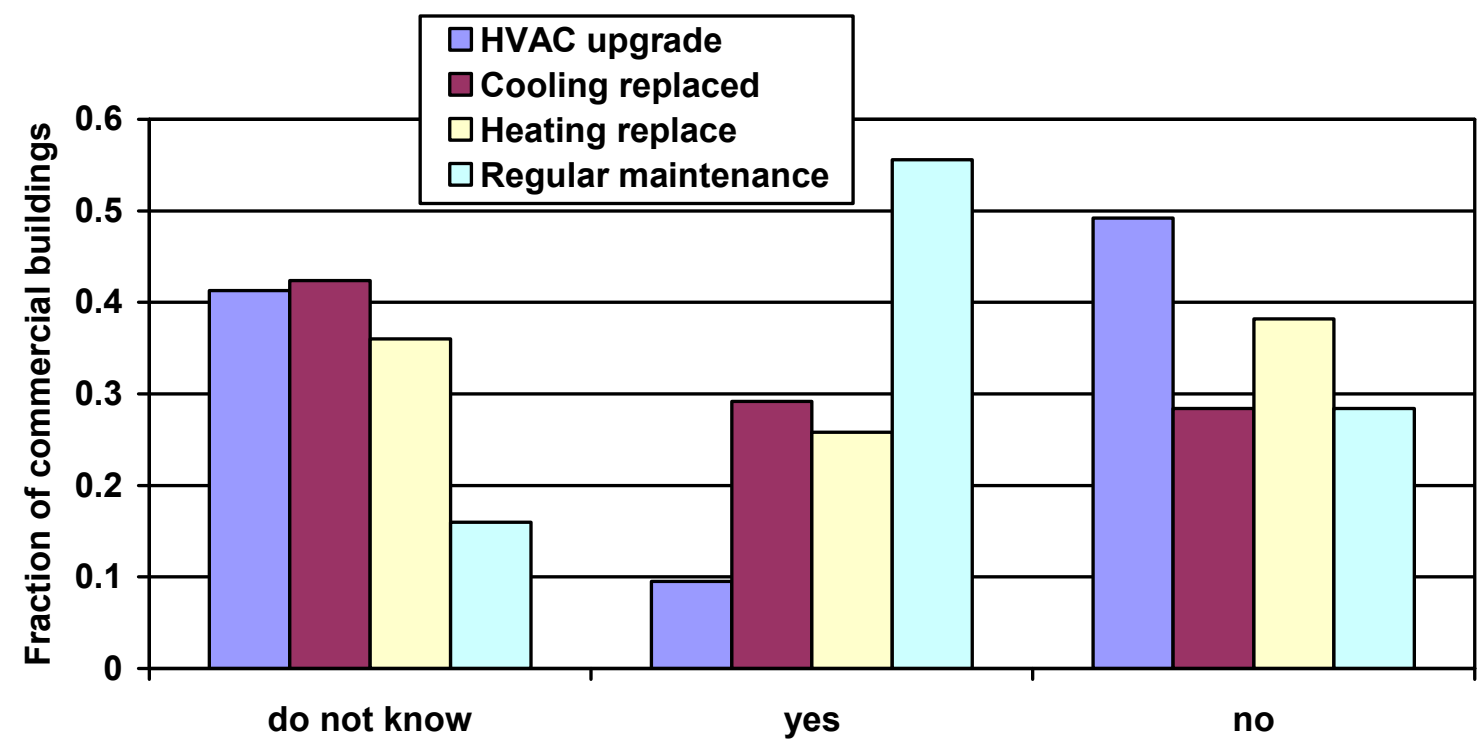

Figure C-5 Discrete PDFs for 2003 CBECS Variables: HVAC Upgraded, Cooling Replaced, Heating Replaced, Maintenance (Raw; All Building Regardless of Percent Conditioned)

The rest of this section documents details for how the performance parameters were modeled for various HVAC components.

COP values from Table C-32 and a simple degradation model based on age are used to model DX coil performance. These values are estimated by approximating the trends observed across historical versions of Standard 90.1. 
Table C-32 DX Coil COP Values at Time of Initial Installation for Different Size Ranges

\begin{tabular}{|l|c|c|c|c|}
\hline \multirow{2}{*}{ Cooling Capacity } & \multicolumn{4}{|c|}{ Year of Installation } \\
\cline { 2 - 5 } & pre-1980 & $\mathbf{1 9 8 0}$ to 1990 & $\mathbf{1 9 9 0}$ to 1998 & $\mathbf{1 9 9 8}$ to 2003 \\
\hline \hline Small $(<65 \mathrm{kBtu} / \mathrm{h})$ & 2.64 & 2.72 & 3.22 & 3.72 \\
\hline Medium $(>65 \mathrm{kBtu} ;<135 \mathrm{kBtu} / \mathrm{h})$ & 2.52 & 2.61 & 2.72 & 2.84 \\
\hline Large $(>135 \mathrm{kBtu} ;<240 \mathrm{kBtu} / \mathrm{h})$ & 2.05 & 2.40 & 2.53 & 2.67 \\
\hline Very large $(>240 \mathrm{kBtu} / \mathrm{h})$ & 1.99 & 2.34 & 2.48 & 2.61 \\
\hline
\end{tabular}

The elapsed time and degradation rate, which vary with the value of MAINT8, are used to model the performance degradation in DX coils. If MAINT8 = "no" or "don't know," the performance degrades at a simple rate of $1 \%$ per year. If MAINT $8=$ "yes," the performance degrades at a simple rate of $0.25 \%$ per year. These rates are assumptions, and further research is needed to better understand how to model this phenomenon. So, for example, if a medium-size DX coil were installed in 1984 (and not replaced) and not maintained, it would have an initial COP of 2.61 and a modeled COP of $2.61 \times(1-(2003-1984) \times$ 0.01 ) or a COP of 2.11 .

\section{Central Chiller}

The initial COP values in Table C-33 and a simple model for performance degradation are used to model the COP of central water-to-water chillers. These values do not include energy consumed by separate heat rejection towers. All central chillers are modeled as water cooled however many, if not most, buildings actually have air-cooled chillers with lower COPs. There is considerable room to improve the modeling of chilled water systems.

Table C-33 Chiller COP Values at Time of Initial Installation for Different Size Ranges

\begin{tabular}{|l|c|c|c|c|}
\hline \multicolumn{1}{|c|}{ Cooling Capacity } & \multicolumn{4}{c|}{ Year of Installation } \\
\hline & pre-1980 & $\mathbf{1 9 8 0}$ to $\mathbf{1 9 9 0}$ & $\mathbf{1 9 9 0}$ to $\mathbf{1 9 9 8}$ & $\mathbf{1 9 9 8}$ to $\mathbf{2 0 0 3}$ \\
\hline Small (<150 tons) & 3.9 & 4.1 & 4.2 & 4.45 \\
\hline Medium (>150 tons; $<300$ tons) & 4.1 & 4.4 & 4.6 & 4.9 \\
\hline Large (> 300 tons) & 4.5 & 4.8 & 5.0 & 5.5 \\
\hline
\end{tabular}

The elapsed time and degradation rate, which vary with the value of MAINT8, are used to model the performance degradation in chillers. If MAINT8 = "no" or "don't know," the performance degrades at a simple rate of $1 \%$ per year. If MAINT $8=$ "yes," the performance degrades at a simple rate of $0.25 \%$ per year. These rates are assumptions, and further research is needed to better understand how to model this phenomenon.

\section{Boiler}

The initial efficiency values in Table C-34 and a simple model for degradation over time are used to model boiler performance in central hot water plant systems. 
Table C-34 Boiler Efficiency Values at Time of Initial Installation

\begin{tabular}{|l|c|c|c|c|}
\hline \multirow{2}{*}{} & \multicolumn{4}{|c|}{ Year of Installation } \\
\cline { 2 - 5 } & pre-1980 & $\mathbf{1 9 8 0}$ to 1990 & $\mathbf{1 9 9 0}$ to 1998 & 1998 to 2003 \\
\hline \hline Boiler efficiency & 0.7 & 0.75 & 0.8 & 0.83 \\
\hline
\end{tabular}

The elapsed time and degradation rate, which vary with the value of MAINT8, are used to model the performance degradation of boiler efficiency. If MAINT8 = "no" or "don't know," the performance degrades at a simple rate of $0.5 \%$ per year. If MAINT8 = "yes," the performance degrades at a simple rate of $0.2 \%$ per year. These rates are assumptions, and further research is needed to better understand how to model this phenomenon.

\section{Heat Pump Heating}

COP values from Table C-35 and a simple degradation model based on age are used to model air-to-air heat pump heating performance.

Table C-35 Heat Pump COP Values at Time of Initial Installation for Different Size Ranges

\begin{tabular}{|l|c|c|c|c|}
\hline \multicolumn{1}{|c|}{ Heating Capacity } & \multicolumn{4}{|c|}{ Year of Installation } \\
\hline & pre-1980 & $\mathbf{1 9 8 0}$ to $\mathbf{1 9 9 0}$ & $\mathbf{1 9 9 0}$ to $\mathbf{1 9 9 8}$ & $\mathbf{1 9 9 8}$ to $\mathbf{2 0 0 3}$ \\
\hline \hline Small $(<65 \mathrm{kBtu})$ & 2.8 & 3.0 & 3.2 & 3.4 \\
\hline Medium $(>65 \mathrm{kBtu} ;<135 \mathrm{kBtu})$ & 2.6 & 2.8 & 3.0 & 3.2 \\
\hline Large $(>135 \mathrm{kBtu} ;<240 \mathrm{kBtu})$ & 2.5 & 2.7 & 2.9 & 3.1 \\
\hline Very large $(>240 \mathrm{kBtu})$ & 2.4 & 2.6 & 2.8 & 3.0 \\
\hline
\end{tabular}

The elapsed time and degradation rate, which vary with the value of MAINT8, are used to model the performance degradation in heat pump coils. If MAINT8 = "no" or "don't know," the performance degrades at a simple rate of $1 \%$ per year. If MAINT8 = "yes," the performance degrades at a simple rate of $0.25 \%$ per year. These rates are assumptions, and further research is needed to better understand how to model this phenomenon.

\section{Constant Volume Fan}

The initial efficiency values in Table C-36 and a simple model for degradation over time are used to model fan performance in packaged constant volume systems. After reviewing the methodology, we determined that the fan efficiencies are not understood very well and are likely too high.

Table C-36 Constant Volume Fan Efficiency Values at Time of Initial Installation

\begin{tabular}{|l|c|c|c|c|}
\hline & \multicolumn{4}{|c|}{ Year of Installation } \\
\cline { 2 - 5 } & pre-1980 & $\mathbf{1 9 8 0}$ to 1990 & $\mathbf{1 9 9 0}$ to 1998 & 1998 to 2003 \\
\hline \hline Fan total efficiency & 0.48 & 0.52 & 0.56 & 0.6 \\
\hline
\end{tabular}

The elapsed time and degradation rate, which vary with the value of MAINT8, are used to model the performance degradation of fan total efficiency. If MAINT8 = "no" or "don't know," the performance degrades at a simple rate of $0.5 \%$ per year. If MAINT $8=$ "yes," the performance degrades at a simple rate of $0.2 \%$ per year. These rates are assumptions, and further research is needed to better understand how to model this phenomenon. So, for example, if a fan was installed in 1984 (and not replaced) and not maintained, it would have an initial efficiency of 0.52 and a modeled efficiency of $0.52 \times(1-(2003-$ $1984) \times 0.005$ ) or a total fan efficiency of 0.47 . 


\section{Variable-Volume Fan}

The initial efficiency values in Table C-37 and a simple model for degradation over time are used to model fan performance for variable-speed fans. After reviewing the methodology, we determined that the fan efficiencies were likely too high and lower estimates should be made for future work; these are listed in the "future revision" row.

Table C-37 Variable-Volume Fan Efficiency Values at Time of Initial Installation

\begin{tabular}{|l|c|c|c|c|}
\hline \multirow{2}{*}{} & \multicolumn{4}{|c|}{ Year of Installation } \\
\cline { 2 - 5 } & pre-1980 & $\mathbf{1 9 8 0}$ to 1990 & $\mathbf{1 9 9 0}$ to $\mathbf{1 9 9 8}$ & $\mathbf{1 9 9 8}$ to 2003 \\
\hline Fan total efficiency & 0.55 & 0.60 & 0.65 & 0.7 \\
\hline Future revision & 0.20 & 0.25 & 0.30 & 0.35 \\
\hline
\end{tabular}

The elapsed time and degradation rate, which vary with the value of MAINT8, are used to model the performance degradation of fan total efficiency. If MAINT8 = "no" or "don't know," the performance degrades at a simple rate of $0.5 \%$ per year. If MAINT8 = "yes," the performance degrades at a simple rate of $0.2 \%$ per year. These rates are assumptions, and further research is needed to better understand how to model this phenomenon.

\section{Gas Heating Coil}

The efficiency values in Table C-38 and a simple model for degradation over time are used to model gas heating coil performance in packaged constant-volume systems.

Table C-38 Natural Gas Heating Coil Efficiency Values at Time of Initial Installation

\begin{tabular}{|l|c|c|c|c|}
\hline & \multicolumn{4}{|c|}{ Year of Installation } \\
\hline & pre-1980 & 1980 to 1990 & 1990 to 1998 & 1998 to 2003 \\
\hline \hline Gas heating coil efficiency & 0.7 & 0.75 & 0.78 & 0.8 \\
\hline
\end{tabular}

The elapsed time and degradation rate, which vary with the value of MAINT8, are used to model the performance degradation of gas heating coils. If MAINT $8=$ "no" or "don't know," the performance degrades at a simple rate of $0.2 \%$ per year. If MAINT $8=$ "yes," the performance degrades at a simple rate of $0.1 \%$ per year. These rates are assumptions, and further research is needed to better understand how to model this phenomenon.

\section{C.20 Outside Air Mechanical Ventilation}

For the modeling, a rate for outside air ventilation must be determined. This is a minimum rate, and more outside air may be introduced if there is an air-side economizer cycle. The rate is used during sizing routines to account for conditioning outside air when sizing equipment. Additional outside air will be introduced by separate infiltration modeling. If a building's system does not include mechanical ventilation, infiltration will be the only source of ventilation air. When the type of HVAC system selected for the modeling tends to include the components for mechanically ventilating the building, the models include components that bring in outside air. This air is introduced through air systems and generally involves air handling units (AHUs) or rooftop packaged units. The purpose of the outside air is to provide healthy indoor air by gradually purging stale indoor air with outdoor air.

Additional research is needed to fully characterize the ventilation rates for outdoor air; however, efforts to do so have been recorded in the literature. Most such efforts combine mechanical ventilation and infiltration. The modeling in the current study uses a separate method of modeling infiltration (see Section C.16). 
Turk et al. (1989) used tracer gas techniques to measure ventilation rates in 38 commercial buildings in the Pacific Northwest. They found mean ventilation rates of $1.5 \mathrm{ACH}$ or $28 \mathrm{l} / \mathrm{s} \cdot$ person and provided a breakdown for educational, libraries, small and large offices, and multi-use. We used these results (see Table 2-8) to model ventilation by assuming that the infiltration portion is equivalent to $9.3 \mathrm{l} / \mathrm{s} \cdot$ person. We then subtracted this rate for uncontrolled ventilation from the whole-building ventilation rates reported by Turk et al. to assemble the default rates for mechanical ventilation listed in Table C-39. For activity types not covered, we used the mean rate of $28-9.3$ or $18.8 \mathrm{l} / \mathrm{s} \cdot$ person. These rates are generally in excess of the minimum levels specified in ASHRAE Standard 62-2004, except for education, which is close but slightly low. We used the rates listed in Table C-39 as the minimum outside air rates for each type of HVAC system that includes an outdoor air mixer. This method of separating infiltration was developed prior to, and independent of, the infiltration input modeling (see Section C.16). Future efforts to separate out infiltration rates from measured overall rates should use results from the infiltration modeling rather than the crude method here based on $1 / \mathrm{s} \cdot$ person. Future efforts to measure outside air rates should seek to separate the portion attributed to infiltration or provide form and fabric data sufficient to model infiltration rates in the measured buildings.

Table C-39 Default Outside Air Mechanical Ventilation Rates by PBA

\begin{tabular}{|c|l|c|c|l|}
\hline $\begin{array}{c}\text { PBA } \\
\text { Code }\end{array}$ & \multicolumn{1}{|c|}{ PBA Name } & $\begin{array}{c}\text { Default Outside } \\
\text { Air Rate } \\
\text { (cfm/person) }\end{array}$ & $\begin{array}{c}\text { Default Outside } \\
\text { Air Rate } \\
\text { (I/s·person) }\end{array}$ & Data Source \\
\hline 1 & Vacant & 0.0 & 0.0 & assumption \\
\hline 2 & Office/professional $<9300 \mathrm{~m}^{2}$ & 55.3 & 26.1 & Turk et al. 1989 \\
\hline 2 & Office/professional $>9300 \mathrm{~m}^{2}$ & 45.3 & 21.4 & Turk et al. 1989 \\
\hline 4 & Laboratory & 39.6 & 18.7 & Turk et al. 1989 \\
\hline 5 & Nonrefrigerated warehouse & 10.6 & 5.0 & assumption \\
\hline 6 & Food sales & 39.6 & 18.7 & Turk et al. 1989 \\
\hline 7 & Public order and safety & 39.6 & 18.7 & Turk et al. 1989 \\
\hline 8 & Outpatient health care & 39.6 & 18.7 & Turk et al. 1989 \\
\hline 11 & Refrigerated warehouse & 4.2 & 2.0 & assumption \\
\hline 12 & Religious worship & 39.6 & 18.7 & Turk et al. 1989 \\
\hline 13 & Public assembly & 39.6 & 18.7 & Turk et al. 1989 \\
\hline 14 & Education & 13.4 & 6.3 & Turk et al. 1989 \\
\hline 15 & Food service & 39.6 & 18.7 & Turk et al. 1989 \\
\hline 16 & Inpatient health care & 39.6 & 18.7 & Turk et al. 1989 \\
\hline 17 & Skilled nursing & 39.6 & 18.7 & Turk et al. 1989 \\
\hline 18 & Lodging & 39.6 & 18.7 & Turk et al. 1989 \\
\hline 25 & Retail & 39.6 & 18.7 & Turk et al. 1989 \\
\hline 26 & Service & 39.6 & 18.7 & Turk et al. 1989 \\
\hline 91 & Other & 18.7 & Turk et al. 1989 \\
\hline
\end{tabular}

\section{C.21 Service Water Heating}

This section describes how service water heating systems were modeled in the study. Hot water is used in commercial buildings for hand washing, cooking, cleaning, showers, etc. 2003 CBECS provides the following qualitative variables related to service water heating (sometimes referred to as domestic hot water):

- Water heating equipment present (WTHTEQ8)

- $\quad$ Large amounts of hot water used (HWTRM8)

- Laundry onsite (LAUNDR8) 
- $\quad$ Type of fuel used for water heating

- Electricity (ELWATR8)

- Natural Gas (NGWATR8)

○ Fuel Oil (FKWATR8)

○ Propane (PRWATR8)

○ District Hot Water (DHWATR8)

○ District Steam (STWATR8).

Chapter 49 of ASHRAE's 2003 HVAC Applications Handbook (ASHRAE 2003) provides limited guidance about service water heating in commercial buildings. This reference is fairly limited, but is the best source of input data identified to date. We used this chapter's recommendations for the quantity of hot water used in commercial buildings, as well as the storage volume and recovery rates. Table C-40 lists the input data based on per-person-per-day. Table C-41 lists the input data for lodging on a per-room basis. 
Table C-40 SHW Inputs per Person per Day by PBA: SI Units

\begin{tabular}{|c|c|c|c|c|c|}
\hline $\begin{array}{l}\text { PBA } \\
\text { Code }\end{array}$ & PBA Name & $\begin{array}{l}\text { Use Rate Value } \\
\text { (I/Person·Day) }\end{array}$ & $\begin{array}{c}\text { Storage } \\
\text { Value } \\
\text { (I/Person) } \\
\end{array}$ & $\begin{array}{c}\text { Water Heating } \\
\text { Recovery Rate } \\
\text { (ml/s·Person) } \\
\end{array}$ & Data Source \\
\hline 1 & Vacant & 0.0 & 6.0 & 0.2 & Assumption \\
\hline 2 & Office/professional & 3.8 & 6.0 & 0.2 & $\begin{array}{l}\text { ASHRAE } 2003 \\
\text { HVAC } \\
\text { Applications }\end{array}$ \\
\hline 4 & Laboratory & 38.0 & 30.0 & 2.0 & Assumption \\
\hline 5 & $\begin{array}{l}\text { Nonrefrigerated } \\
\text { warehouse }\end{array}$ & 2.3 & 6.0 & 0.2 & Assumption \\
\hline 6 & Food sales & 19.0 & 30.0 & 1.0 & Assumption \\
\hline 7 & $\begin{array}{l}\text { Public order and } \\
\text { safety }\end{array}$ & 3.8 & 6.0 & 0.2 & Assumption \\
\hline 8 & $\begin{array}{l}\text { Outpatient health } \\
\text { care }\end{array}$ & 11.4 & 18.0 & 0.6 & Assumption \\
\hline 11 & $\begin{array}{l}\text { Refrigerated } \\
\text { warehouse }\end{array}$ & 2.3 & 5.0 & 0.2 & Assumption \\
\hline 12 & Religious worship & 1.0 & 2.0 & 0.1 & Assumption \\
\hline 13 & Public assembly & 1.0 & 2.0 & 0.1 & Assumption \\
\hline 14 & Education: college & 2.3 & 6.0 & 0.2 & Assumption \\
\hline 14 & $\begin{array}{l}\text { Education: } \\
\text { elementary school }\end{array}$ & 2.3 & 6.0 & 0.2 & $\begin{array}{l}\text { ASHRAE } 2003 \\
\text { HVAC } \\
\text { Applications }\end{array}$ \\
\hline 14 & $\begin{array}{l}\text { Education: high } \\
\text { school }\end{array}$ & 6.8 & 12.0 & 0.4 & $\begin{array}{l}\text { ASHRAE } 2003 \\
\text { HVAC } \\
\text { Applications }\end{array}$ \\
\hline 14 & $\begin{array}{l}\text { Education: } \\
\text { preschool }\end{array}$ & 2.3 & 6.0 & 0.2 & Assumption \\
\hline 15 & Food service & 38.0 & 60.0 & 2.0 & Assumption \\
\hline 16 & $\begin{array}{l}\text { Inpatient health } \\
\text { care }\end{array}$ & 11.4 & 18.0 & 0.6 & Assumption \\
\hline 17 & Skilled nursing & 11.4 & 18.0 & 0.6 & Assumption \\
\hline 25 & Retail & 2.3 & 4.0 & 0.2 & Assumption \\
\hline 26 & Service & 3.8 & 6.0 & 0.2 & Assumption \\
\hline 91 & Other & 3.8 & 6.0 & 0.2 & Assumption \\
\hline
\end{tabular}


Table C-41 SHW Inputs per Room per Day for Lodging by PBA: SI Units

\begin{tabular}{|c|l|c|c|c|c|}
\hline $\begin{array}{c}\text { PBA } \\
\text { Code }\end{array}$ & \multicolumn{1}{|c|}{ PBA Name } & $\begin{array}{c}\text { Use Rate Value } \\
\text { (I/ Room·Day) }\end{array}$ & $\begin{array}{c}\text { Storage } \\
\text { Value } \\
\text { (I/Room) }\end{array}$ & $\begin{array}{c}\text { Water Heating } \\
\text { Recovery Rate } \\
\text { (ml/s-Room) }\end{array}$ & Data Source \\
\hline \hline 18 & $\begin{array}{l}\text { Lodging: 20 or } \\
\text { fewer rooms }\end{array}$ & 75.8 & 50.0 & 3.0 & $\begin{array}{l}\text { ASHRAE 2003 } \\
\text { HVAC } \\
\text { Applications }\end{array}$ \\
\hline 18 & $\begin{array}{l}\text { Lodging: } 60 \\
\text { rooms }\end{array}$ & 53.1 & 50.0 & 3.0 & $\begin{array}{l}\text { ASHRAE 2003 } \\
\text { HVAC } \\
\text { Applications }\end{array}$ \\
\hline 18 & $\begin{array}{l}\text { Lodging: } 100 \text { or } \\
\text { more rooms }\end{array}$ & 37.9 & 50.0 & 3.0 & $\begin{array}{l}\text { ASHRAE 2003 } \\
\text { HVAC } \\
\text { Applications }\end{array}$ \\
\hline
\end{tabular}

\section{C.22 Refrigeration}

This energy use category is for commercial refrigeration used for cold storage and display, and does not include residential style refrigerators.

CBECS variables of interest include:

- Refrigeration equipment used (RFGEQP8)

- Number of closed refrigerated cases (RFGCLN8)

- Number of open refrigerated cases (RFGOPN8)

- Number of walk-in refrigeration units (RFGWIN8)

The number of cases and walk-in units is interesting, but we found that developing quantitative descriptions of the refrigeration systems from the data available in CBECS was difficult. We therefore adopted a simple modeling approach and used CEUS data (CEC 2006) listed in Table 2-1 to define refrigeration loads for all buildings that responded that refrigeration equipment was being used (RFGEQP8 = 1). The CEUS data for refrigeration EUI were processed to derive power densities by assuming constant operation year round. The modeling inputs are listed in Table C-42. The refrigeration systems were modeled as an external equipment load rather than the more complex refrigeration component models that are also available in EnergyPlus. This simplification ignores the interactions between refrigeration systems and thermal loads where cooling loads decrease and spaces may be controlled to lower humidity levels to avoid condensation. 
Table C-42 Refrigeration Power Densities

\begin{tabular}{|c|l|c|c|}
\hline $\begin{array}{c}\text { PBA } \\
\text { Code }\end{array}$ & \multicolumn{1}{|c|}{ PBA } & $\begin{array}{c}\text { Refrigeration } \\
\text { Power Density } \\
\left(\mathbf{W} / \mathbf{f t}^{2} \mathbf{)}\right.\end{array}$ & $\begin{array}{c}\text { Refrigeration } \\
\text { Power Density } \\
\left(\mathbf{W} / \mathbf{m}^{2} \text { ) }\right.\end{array}$ \\
\hline \hline 1 & Vacant & 0.00 & 0.0 \\
\hline 2 & Office/professional $<30,000 \mathrm{ft}^{2}$ & 0.07 & 0.8 \\
\hline 2 & Office/professional $>30,000 \mathrm{ft}^{2}$ & 0.06 & 0.6 \\
\hline 4 & Laboratory & 0.28 & 3.0 \\
\hline 5 & Nonrefrigerated warehouse & 0.05 & 0.5 \\
\hline 6 & Food sales & 2.60 & 28.0 \\
\hline 7 & Public order and safety & 0.06 & 0.6 \\
\hline 8 & Outpatient health care & 0.08 & 0.9 \\
\hline 11 & Refrigerated warehouse & 1.53 & 16.5 \\
\hline 12 & Religious worship & 0.03 & 0.3 \\
\hline 13 & Public assembly & 0.03 & 0.3 \\
\hline 14 & Education & 0.06 & 0.6 \\
\hline 15 & Food service & 1.12 & 12.1 \\
\hline 16 & Inpatient health care & 0.08 & 0.9 \\
\hline 17 & Skilled nursing & 0.08 & 0.9 \\
\hline 18 & Lodging & 0.14 & 1.5 \\
\hline 25 & Retail & 0.15 & 1.6 \\
\hline 26 & Service & 0.12 & 1.3 \\
\hline 91 & Other & 0.10 & 1.1 \\
\hline
\end{tabular}

\section{C.23 Gas Appliance Intensity}

For internal loads powered by natural gas, we adopted a simple modeling approach and used CEUS data (CEC 2006) listed in Table 2-3 to define gas appliance loads for all buildings. The CEUS data for gas appliance EUI were processed to derive mean power densities by assuming constant, year-round operation. The modeling inputs were developed using a Gaussian distribution with the mean and standard deviations varying by PBA as listed in Table C-43. The standard deviations were developed by assuming them to be $1 / 15$ of the mean and may be rather low. 
Table C-43 Interior Gas Appliance Intensity Assignments

\begin{tabular}{|c|l|c|c|c|c|}
\hline $\begin{array}{c}\text { PBA } \\
\text { Code }\end{array}$ & \multicolumn{1}{|c|}{ PBA Name } & $\begin{array}{c}\text { Mean Power } \\
\text { Density } \\
\left(\mathbf{W} / \mathbf{f t}^{\mathbf{}} \mathbf{)}\right.\end{array}$ & $\begin{array}{c}\text { Standard } \\
\text { Deviation } \\
\left(\mathbf{W} / \mathbf{f t}^{2}\right)\end{array}$ & $\begin{array}{c}\text { Mean Power } \\
\text { Density } \\
\left(\mathbf{W} / \mathbf{m}^{\mathbf{2}} \mathbf{)}\right.\end{array}$ & $\begin{array}{c}\text { Standard } \\
\text { Deviation } \\
\left(\mathbf{W} / \mathbf{m}^{2} \mathbf{)}\right.\end{array}$ \\
\hline \hline 1 & Vacant & 0.00 & 0.000 & 0.05 & 0.003 \\
\hline 2 & Office/professional & 0.04 & 0.002 & 0.40 & 0.027 \\
\hline 4 & Laboratory & 3.72 & 0.248 & 40.00 & 2.667 \\
\hline 5 & lonrefrigerated warehouse & 0.00 & 0.000 & 0.00 & 0.000 \\
\hline 6 & Food sales & 0.35 & 0.023 & 3.74 & 0.249 \\
\hline 7 & Public order/safety & 0.09 & 0.006 & 1.00 & 0.067 \\
\hline 8 & Outpatient health care & 0.33 & 0.022 & 3.56 & 0.237 \\
\hline 11 & Refrigerated warehouse & 0.13 & 0.009 & 1.44 & 0.096 \\
\hline 12 & Religious worship & 0.04 & 0.002 & 0.40 & 0.027 \\
\hline 13 & Public assembly & 0.04 & 0.002 & 0.40 & 0.027 \\
\hline 14 & Education & 0.04 & 0.003 & 0.43 & 0.029 \\
\hline 15 & Food service & 5.14 & 0.342 & 55.30 & 3.687 \\
\hline 16 & Inpatient health care & 0.33 & 0.022 & 3.56 & 0.237 \\
\hline 17 & Skilled nursing & 0.33 & 0.022 & 3.56 & 0.237 \\
\hline 18 & Lodging & 0.20 & 0.014 & 2.19 & 0.146 \\
\hline 25 & Retail (except malls) & 0.03 & 0.002 & 0.28 & 0.019 \\
\hline 26 & Service (except food) & 0.22 & 0.015 & 2.37 & 0.158 \\
\hline 91 & Other & 0.09 & 0.006 & 1.00 & 0.067 \\
\hline
\end{tabular}




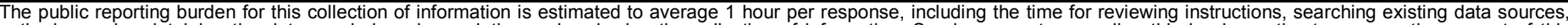

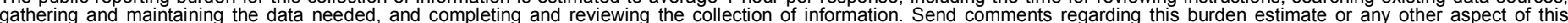

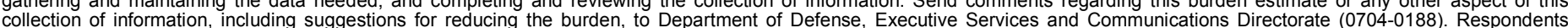

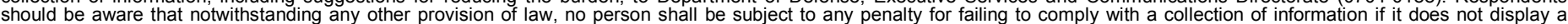

should be aware that notwithstanding

PLEASE DO NOT RETURN YOUR FORM TO THE ABOVE ORGANIZATION.

\begin{tabular}{l|l|l|l} 
1. REPORT DATE $(D D-M M-Y Y Y Y)$ & 2. REPORT TYPE & 3. DATES COVERED (FrOm - TO)
\end{tabular}

March 2008

Technical Report

4. TITLE AND SUBTITLE

Methodology for Modeling Building Energy Performance across the

Commercial Sector

5a. CONTRACT NUMBER

DE-AC36-99-G010337

5b. GRANT NUMBER

5c. PROGRAM ELEMENT NUMBER

6. AUTHOR(S)

B. Griffith, N. Long, P. Torcellini, R. Judkoff, D. Crawley, and J.

Ryan

5d. PROJECT NUMBER

NREL/TP-550-41956

5e. TASK NUMBER

BEC71006

5f. WORK UNIT NUMBER
7. PERFORMING ORGANIZATION NAME(S) AND ADDRESS(ES)

National Renewable Energy Laboratory

1617 Cole Blvd.

Golden, CO 80401-3393
8. PERFORMING ORGANIZATION REPORT NUMBER

NREL/TP-550-41956

9. SPONSORING/MONITORING AGENCY NAME(S) AND ADDRESS(ES)

10. SPONSOR/MONITOR'S ACRONYM(S) NREL

11. SPONSORING/MONITORING AGENCY REPORT NUMBER

12. DISTRIBUTION AVAILABILITY STATEMENT

National Technical Information Service

U.S. Department of Commerce

5285 Port Royal Road

Springfield, VA 22161

\section{SUPPLEMENTARY NOTES}

14. ABSTRACT (Maximum 200 Words)

This report uses EnergyPlus simulations of each building in the 2003 Commercial Buildings Energy Consumption Survey (CBECS) to document and demonstrate bottom-up methods of modeling the entire U.S. commercial buildings sector (EIA 2006). The ability to use a whole-building simulation tool to model the entire sector is of interest because the energy models enable us to answer subsequent "what-if" questions that involve technologies and practices related to energy. This report documents how the whole-building models were generated from the building characteristics in 2003 CBECS and compares the simulation results to the survey data for energy use.

15. SUBJECT TERMS

commercial building; building technology; cbecs; whole-building simulation tool

16. SECURITY CLASSIFICATION OF:
\begin{tabular}{l|l|l|}
\hline $\begin{array}{l}\text { a. REPORT } \\
\text { Unclassified }\end{array}$ & $\begin{array}{c}\text { b. ABSTRACT } \\
\text { Unclassified }\end{array}$ & Unclassified \\
\end{tabular}

\begin{tabular}{l|l} 
17. LIMITATION & 18. $\begin{array}{l}\text { NUMBER } \\
\text { OF ABSTRACT } \\
\text { OF PAGES } \\
\text { UL }\end{array}$
\end{tabular}

19a. NAME OF RESPONSIBLE PERSON

19b. TELEPHONE NUMBER (Include area code) 